\title{
Le théâtre de Koffi Kwahulé. Une nouvelle mythologie urbaine
}

\author{
par
}

\section{Caroline Barrière}

Thèse soumise à la Faculty of Graduate Studies and Research Comme exigence partielle en vue de l'obtention de la maîtrise en français (Master of Arts)

Department of French

Carleton University

Ottawa, Ontario

(C) 2011, Caroline Barrière 
Library and Archives Canada

Published Heritage Branch

395 Wellington Street Ottawa ON K1A ON4 Canada
Bibliothèque et

Archives Canada

Direction du

Patrimoine de l'édition

395, rue Wellington

Ottawa ON K1A 0N4

Canada
Your file Votre référence
ISBN: $978-0-494-79571-2$
Our file Notre reférence
ISBN: $978-0-494-79571-2$
NOTICE:

The author has granted a nonexclusive license allowing Library and Archives Canada to reproduce, publish, archive, preserve, conserve, communicate to the public by telecommunication or on the Internet, loan, distribute and sell theses worldwide, for commercial or noncommercial purposes, in microform, paper, electronic and/or any other formats.

The author retains copyright ownership and moral rights in this thesis. Neither the thesis nor substantial extracts from it may be printed or otherwise reproduced without the author's permission.
AVIS:

L'auteur a accordé une licence non exclusive permettant à la Bibliothèque et Archives Canada de reproduire, publier, archiver, sauvegarder, conserver, transmettre au public par télécommunication ou par l'Internet, prêter, distribuer et vendre des thèses partout dans le monde, à des fins commerciales ou autres, sur support microforme, papier, électronique et/ou autres formats.

L'auteur conserve la propriété du droit d'auteur et des droits moraux qui protège cette thèse. Ni la thèse ni des extraits substantiels de celle-ci ne doivent être imprimés ou autrement reproduits sans son autorisation.
In compliance with the Canadian Privacy Act some supporting forms may have been removed from this thesis.

While these forms may be included in the document page count, their removal does not represent any loss of content from the thesis.
Conformément à la loi canadienne sur la protection de la vie privée, quelques formulaires secondaires ont été enlevés de cette thèse.

Bien que ces formulaires aient inclus dans la pagination, il n'y aura aucun contenu manquant. 


\section{RÉSUMÉ}

Cette étude traite de six pièces de Koffi Kwahulé, un auteur de la Côte d'Ivoire qui vit en France. D'abord, nous réfléchissons à l'africanité et au fait d'être Noir, à travers les yeux de quelqu'un qui a quitté son pays natal il y a 30 ans. Dans ces circonstances, que signifient les notions d'africanité et de Noir? Le théâtre de Kwahulé met en lumière ce dilemme devenu une part importante du dialogue culturel en France. Sa réflexion s'articule autour de stratégies dramaturgiques qui consistent à subvertir les mythologies fondatrices de la culture européenne: la Bible, le mythe de Faust et les rituels de l'Église catholique, en transformant leur signification au sein des problématiques contemporaines du racisme et de l'immigration. Nous voyons qu'à travers ces rituels contemporains de pouvoir, qui culminent souvent par la mort, l'auteur construit une nouvelle mythologie urbaine qui représente le paradigme des exclus de la société française.

Mots clés: Cette vieille magie noire - Bintou - Ave Maria - Big Shoot - Blue-S-cat - Misterioso119 - africanité - Noir - théâtre francophone - cérémonies de pouvoir 


\section{ABSTRACT}

This study deals with six plays by Koffi Kwahulé, a writer from the Ivory Coast living in France. Firstly, we reflect on the ideas of Africanity and Blackness, as seen through the eyes of someone who has been living away from his place of birth for 30 years. What do notions associated with Africanity and being Black mean in these circumstances? Kwahulé's theatre reflects this dilemma,which has become an important part of the cultural dialogue in France. His reflexion is filtered through his dramaturgical strategies which consist of subverting the founding mythologies of European culture: the Bible, the Myth of Faust, the rituals of the Catholic church and shifting their meaning to contemporary issues of racism and immigration. We learn that through these contemporary rituals of power, often ending in death, the author constructs a new urban mythology that represents the paradigm of the excluded of French society.

Key words: Cette vieille magie noire - Bintou - Ave Maria - Big Shoot - Blue-S-cat Misterioso-119 - africanity - Black - francophone theatre - ceremonies of power 


\section{DÉDICACE}

À Paul, merci pour TOUT 


\section{REMERCIEMENTS}

Un immense merci à ma directrice de thèse, Madame Alvina Ruprecht, qui m'a été d'un immense secours tout au long des nombreuses années qui ont été nécessaires pour compléter cette thèse. Il n'a pas été toujours facile de mettre de côté mes réflexes d'écriture journalistique pour arriver à donner vie à cette étude. Je comprends désormais ce que Boileau a voulu signifier quand il a écrit: Vingt fois sur le métier remettez votre ouvrage...

Je tiens également à remercier Paul Roux qui m'a littéralement poussée à terminer ma thèse alors que le cœur n'y était pas toujours. Merci pour ton immense patience et les innombrables corrections. Cette thèse est un peu la tienne... Merci à Angélique qui, du haut de ses quatre ans, a été formidable.

Merci à Koffi Kwahulé quı a toujours fait preuve d'une grande disponibilité en répondant à mes innombrables questions au fils des années.

Un profond merci à Odette Dumas et Ariane Piraveau pour leurs commentaires pertinents et leurs conseils judicieux.

Enfin, je souhaite ici reconnaître l'engagement de mes anciens patrons du journal Le Droit: Michel Gauthier, Jean Gagnon et André Larocque qui m'ont permis de m'absenter du travail à plusieurs reprises entre 2002 et 2009. 


\section{TABLE DES MATIÈRES}

Thesis Acceptance Form

Résumé

iii

Abstract

iv

Dédicace

V

Remerciements

vi

Table des matières

vii

Introduction

1

Chapitre 1: «Africanité» et identité

1.1 Les dramaturges de la «nouvelle africanité» 21

1.2 «Africanité» et langue $\quad 28$

1.3 Le «nouveau» théâtre «afrıcain» 33

$\begin{array}{ll}\text { Chapitre 2: Le mythe subverti } & 38\end{array}$

2.1 Définitions du mythe 39

2.1.1 Le mythe selon Lévi-Strauss $\quad 39$

2.1.2 Le mythe selon Barthes $\quad 42$

2.1.3 Le mythe selon Girard $\quad 44$

2.1.4 Résumé des arguments autour du mythe 46

2.2 Cette vieille magie noire et le mythe de Faust 48

2.2.1 Les multiples fonctions des mises en abîme $\quad 61$

2.3 Bintou et le sacrifice expiatoire $\quad 68$

2.3.1 Le rôle du chœur dans la tragédie $\quad 73$

2.3.2 La perspective de l'immigration $\quad 77$

2.3.3 L'excision: de la tradition au rôle de chef de bande 86 
2.4.2 Messe basse 101

2.4.3 Prière 106

2.4.4 Confessions 107

$\begin{array}{ll}\text { Chapitre 3: La mythologie de la victime } & 117\end{array}$

3.1 Le bouc émissaire et les rituels de pouvoir dans Big Shoot 118

3.2 Le meurtre rituel dans Blue-S-cat 133

3.3 La messe et le meurtre rituel dans Misterioso-119 142

3.3.1 Le rituel en tant que théâtre $\quad 147$

3.3.2 Le rituel de mise à mort 148

3.3.3 Les figures du Mal 156

Conclusion

Bibliographie

Appendices

A) Théâtrographie de Koffi Kwahulé

i

B) Entretien inédit avec Koffi Kwahulé

ix 


\section{INTRODUCTION}

Ma rencontre avec la dramaturgie de Koffi Kwahulé est le fruit du hasard. Je me suis toujours intéressée au continent africain et, au moment d'entreprendre cette thèse, je savais que le théâtre de cette région allait être au cœur de mes recherches. Je me suis donc mise à lire des pièces de divers auteurs francophones contemporains d'Afrique, des Antilles et de la Caraỉbe. Lors de la lecture de quelques pièces de l'auteur d'origine ivoirienne Koffi Kwahulé, j'ai été frappée par son écriture. À vrai dire, ses textes ne ressemblaient à rien de ce que je connaissais. Il me semblait que mes repères ne m'étaient d'aucune utilité. Plus je lisais, moins j'étais certaine de comprendre ce dont il était question, à un point tel que je me suis dit qu'il fallait que j'explore davantage cet univers dramaturgique qui m'était complètement étranger. J'ai alors compris que cette prise de contact serait d'une grande importance et qu'elle allait influencer, au plus haut point, la direction que prendrait ma thèse.

Tout juste avant d'entreprendre mes études à la maîtrise, j'avais effectué un séjour en Afrique de l'Ouest (Bénin, Burkina Faso, Mali et Sénégal). À Ouagadougou, j'ai pu assister à une représentation, en plein air, de la pièce En attendant le vote des bêtes sauvages, ${ }^{1}$ d'après le roman d'Ahmadou Kourouma. Plus tard, j'ai appris qu'il existait un lien entre Kourouma et la pièce Fama de Koffi Kwahulé. En effet, le dramaturge s'est inspiré des romans Les Soleils des Indépendances et Monnè, outrages et défis de Kourouma pour lui rendre hommage.

De plus, à l'été 2004, j'ai obtenu une bourse de l'Université Carleton pour me rendre en France faire de la recherche et rencontrer François Le Pillouër, directeur du Théâtre National de Bretagne (TNB) - Centre Européen Théâtral et Chorégraphique. Le TNB avait commandé une œuvre à Koffi Kwahulé qui, pour l'occasion, a écrit Misterioso-119. Par la même occasion, j'ai pu 
rencontrer le dramaturge à Paris pour une première fois. Cet entretien inédit est d'ailleurs joint en annexe de ma thèse. C'est à l'occasion de ce voyage que j'ai réellement compris l'importance de Koffi Kwahulé sur la scène contemporaine.

À ce jour, les pièces Big Shoot, Ave Maria, Profils atypiques et Jaz ont été présentées au Québec. Big Shoot a été la seule d'entre elles à être présentée à Ottawa. Il m'avait alors été possible d'assister à une représentation de la pièce à La Nouvelle Scène, en février 2006. Il s'agissait d'une coproduction du Théâtre Denise-Pelletier et de la compagnie Lézards qui bougent, sous la direction de Kristian Frédric. (Voir la théâtrographie)

Par ailleurs, les 11 et 12 avril 2008, l'Université de Paris III - Sorbonne Nouvelle et l'Université de Paris IV ont organisé un colloque international, qui a eu lieu au Lavoir Moderne Parisien, un petit théâtre au sein de la Goutte-d'Or, le quartier africain du $18^{\mathrm{e}}$ arrondissement. Ce colloque auquel j'ai assisté portait justement sur le travail de Koffi Kwahulé, et s'intitulait Kwahulé: frère de son, frère de scène. Voix chœurs à corps. Il rassemblait de nombreux chercheurs dont les communications ont porté sur divers aspects de son travail de création: le jazz, la musicalité, la choralité liée aux corps et aux voix, l'oralité, la tragédie, les pratiques symboliques, l'écriture, la dramaturgie du déracinement, la place de l'Afrique et la question de l'altérité, pour ne mentionner que ces quelques éléments. Plusieurs écrivains, metteurs en scène et acteurs ont également participé aux discussions. Les responsables scientifiques du colloque étaient Sylvie Chalaye ${ }^{2}$ (Paris III) et Virginie Soubrier ${ }^{3}$ (Paris IV). Cet événement m'a beaucoup apporté en m'ouvrant de nombreuses pistes de réflexion. ${ }^{4}$ À cette occasion, j'ai également pu

\footnotetext{
2 Sylvıe Chalaye est l'une des spécıalıstes du théâtre contemporaın d'Afrıque francophone en Europe. Elle est professeure à l'Instıtut d'Études Théâtrales de Parıs III et auteure de plusieurs ouvrages consacrés aux scènes contemporaınes d'Afrıque francophone et à l'image du Noır à travers l'hıstoire Elle est membre du comıté de dırectıon d'Africultures et y collabore depuıs de nombreuses années. Elle collabore également à la revue Théâtre/Publıc, dont elle a coordonné des numéros traitant des écritures contemporaınes en Afrıque noire et des comédıens Noırs de la scène françaıse

3 Virgınıe Soubrier a complété une thèse de doctorat (ınédıte) intitulée Koffi Kwahulé Une volx afro-européenne sur la scène françalse contemporaine

4 Les actes du colloque ont été publiés dans le numéro 77-78 d'Africultures (Vorr la bibliographie)
} 
rencontrer de nouveau Koffi Kwahulé.

Toutefois, le dramaturge demeure, encore aujourd'hui, passablement méconnu du grand public et en marge du théâtre institutionnalisé français, et ce, bien qu'il soit étudié au sein du monde universitaire. Ses pièces sont traduites dans plusieurs langues mais sont surtout jouées en Europe bien que l'Afrique et l'Amérique du Nord s'intéressent de plus en plus à son théâtre. À titre d'exemple, ses pièces sont notamment jouées au Lavoir Moderne Parisien et, depuis plusieurs années, à la Chapelle du Verbe Incarné, lieu de rencontre des Théâtres d'outre-mer en Avignon. C'est dans ce cadre que se retrouvent, notamment, des spectacles et des artistes des Départements et Régions d'Outre-Mer de la Guadeloupe, la Martinique, la Guyane française et La Réunion ainsi que ceux qui sont issus des anciennes colonies françaises. Ces artistes et leurs spectacles sont, en quelque sorte, présentés en marge du Festival d'Avignon (où sont invités les grands noms) et en marge du Off du Festıval (dans les divers lieux de la ville), comme s'ils étaient exclus ${ }^{5}$ de l'ensemble de l'institution théâtrale française et européenne.

La situation de Koffi Kwahulé est donc des plus pertınentes. Il est originaire de la Côte d'Ivoire où il a fait ses études pour devenır comédien, avant d'aller étudier le théâtre à Parıs. Il vit d'ailleurs dans la capitale française depuis plus de 30 ans. De par ses choix, il a pu réfléchir sur ses rapports avec l'Afrique et comment ceux-ci déterminent sa manière de voir le monde. Une réflexion issue de son expérience en France s'est donc transmise à son écriture, pour devenir ce que nous appelons sa vision kwahuléenne du monde. Elle se situe au confluent de nombreux questionnements semblables à travers les diverses réflexions identitaires qui émergent dans le contexte d'une Europe en train de se redéfinir culturellement.

Dans cette thèse, nous voulons montrer comment le théâtre de Koffi Kwahulé est une

5 Toutefors, grâce à Greg Germain et Marıe Pierre Bousquet, les spectacles de Koffi Kwahulé présentés à la Chapelle du Verbe Incarné sont captés par RFO et mis à la dispositıon des chercheurs Par alleurs, la programmation Off de la Chapelle attıre des foules depuis un certain temps et contribue beaucoup à la visibilité de tous ces théâtres À ce sujet, voir l'artıcle d'Alvina Ruprecht «Une tête de pont en Avıgnon La Chapelle du Verbe Incarné et l'ouverture vers le Tout-Monde » Africultures 80-81 (2010) 254-259 
réflexion sur cette identité devenue problématique, à cause de son éloignement de son pays d'origine. Quelles sont les conséquences de cette rupture et de cette vie renouvelée sur son écriture et sa vision artistique? Nous avons également voulu examiner cette question en nous intéressant à la manière dont le dramaturge affirme cette identité transformée par sa manière de subvertir la pensée élaborée dans certains textes fondateurs, comme par exemple, ceux qui relèvent de la mythologie chrétienne et qui symbolisent les fondements de la société européenne dans laquelle il vit. Telle a été la problématique qui a guidé notre étude.

D'un point de vue méthodologique, notre étude est d'abord basée sur les thématiques que nous retrouvons dans le théâtre de Koffi Kwahulé. Elle ne comporte pas une analyse chronologique ni historique de son corpus. Elle est plutôt organisée en fonction de la nature des différentes pièces que nous avons regroupées selon leur esthétique théâtrale scénique et textuelle, qu'il s'agisse d'une esthétique réaliste ou d'une esthétique où il déconstruit les conventions du théâtre européen.

Par ailleurs, même si le théâtre de Koffi Kwahulé est intimement lié au jazz et à la musıcalité, nous avons préféré nous distancier de cette approche et choisi de ne pas faire intervenir une discussion sur les formes héritées de la musique jazz dans son œuvre dramaturgique. Et bien que nous ayons compris que les questions de musique, qui concernent l'esthétique scénique et textuelle, déterminaient le mouvement de certaines pièces, nous avons préféré ne pas aborder ces questions, parce qu'il s'agit d'un domaine qui nous est moins familier. Nous nous contenterons ici de citer le travail de Gilles Mouëllic à ce sujet, et notamment le livre d'entretiens Frères de son, réalisé avec Koffi Kwahulé et qui traite du jazz et de l'écriture jazz dans l'œuvre du dramaturge.

Dans cette étude, nous avons choisi de ne pas faire référence au postcolonialisme. Il ne s'agissait pas d'une manière de rejeter la théorie postcoloniale. Bien au contraire, nous avons compris sa pertinence pour nos analyses. Cependant, nous avons décidé de ne pas théoriser les 
questions concernant le postcolonialisme dans cette thèse. Si nous avions choisi de parler de cette théorie, cela nous aurait obligé à passer beaucoup de temps sur ses ramifications au sein des débats actuels dans les contextes de la littérature africaine et du théâtre postcolonial, deux disciplines fort différentes. Ce choix aurait probablement eu comme conséquence de nous éloigner de l'analyse des textes de Kwahulé.

Lors de notre analyse des pièces, notre intérêt s'est plutôt porté sur différentes thématiques comme l'immigration, le racisme, la violence, les luttes de pouvoir et les problèmes de la banlieue. Nous avons organisé notre analyse à partir de certaines catégories qui révèlent et prolongent sa vision. La logique de ces catégories a prévalu au-delà de la chronologie de sa création. Bien que nous ayons adopté cette approche, nous avons été en mesure de constater une évolution, un changement entre les pièces écrites à ses débuts par rapport à celles qui ont été publiées au cours des dernières années. La manière dont nous avons abordé ces pièces fait en sorte que certaines thématiques se recoupent-les catégories n'étant pas étanches. Nous avons toutefois ciblé la thématique dominante, la catégorie fondamentale, au sein de chacun des textes.

De plus, nous avons eu recours à l'anthropologie culturelle, alors que nous avons examiné certaines pratiques traditionnelles telles que les questions concernant l'excision et le cannibalisme. L'anthropologie a aussi servi à alimenter notre analyse sur les mythes, selon les définitions de Claude Lévi-Strauss, Roland Barthes et René Girard.

Les premières questions qui ont guidé ma réflexion ont été les suivantes: qu'est-ce qu'un auteur africain? Après avoir passé plus de la moitié de sa vie en France, Koffi Kwahulé est-il un auteur africain? Pour tenter de répondre à ces questions, nous avons organisé le plan de notre travail en trois chapitres.

Dans le premier chapitre, nous examinerons la question de l'africanité et de l'identité. En effet, il nous semblait primordial de cerner cette notion d'africanité, celle de l'Afrique en tant que 
lieu géographique tout autant que le concept identitaire associé à la couleur «Noire» ${ }^{6}-« N o i r »$ signifiant en effet une origine africaine. Nous avons d'abord fait le bilan des discours identitaires. Intitulé «Africanité» et identité, notre premier chapitre démontre que certaines questions relatives à l'africanité et à l'identité «Noire» sont, selon notre analyse, des concepts très instables.

Nous y rappellerons certaines idées issues du mouvement de la Négritude, dont Aimé Césaire et Léopold Sédar Senghor ont été de grands défenseurs. Nous y abordons également les écrits de Frantz Fanon qui, pour sa part, a remis en question la notion de Négritude, alors que sa vision s'inspirait plutôt d'une identité intersubjective. Par la suite, nous nous intéresserons au concept d'identité hybride, telle que formulée par Édouard Glissant. Nous passerons ensuite à cette nouvelle génération de dramaturges, lesquels remettent en question leurs rapports identitaires face à l'Afrique, à l'africanité et à la Négritude.

Notre deuxième chapitre s'intitule Le mythe subverti. Pour préparer notre analyse sur la subversion des mythes dans le théâtre de Koffi Kwahulé, nous étudierons les définitions du mythe selon Claude Lévi-Strauss, Roland Barthes et René Girard. En nous basant sur ces définitions, nous verrons comment elles correspondent à la manière dont Koffi Kwahulé utilise, réécrit et, surtout, subvertit ces mythes. Dans ce même chapitre, après avoir parlé du mythe, nous analyserons trois pièces du dramaturge: Cette vieille magie noire, Bintou et Ave Maria. Cette vieille magie noire correspond au mythe de Faust. Le parcours de Bintou représente le Calvaire du Christ et sa crucifixion. Dans la pièce Ave Maria, il est question du mythe de Caïn et Abel, dans l'Ancien Testament. Toutes ces mythologies sont représentées de manière très réaliste dans des contextes contemporains qui correspondent à l'actualité et qui sont facilement identifiables par le public.

Dans le troisième chapitre, intitulé La mythologie de la victime, nous examinerons trois

6 Tout au long de notre analyse, nous emploierons la majuscule avec «Norr» et «Blanc». En ce qui a trait aux passages d'ouvrages cités, nous les reprodurrons tel quel, selon l'emplo1 qui en a été fait par son auteur. 
autres pièces de Koffi Kwahulé: Big Shoot, Blue-S-cat et Misterioso-119. Il sera alors question des cérémonies et des rites de pouvoir. Les travaux de René Girard apporteront un éclairage important à ce segment de notre travail dans le but d'aboutir à une nouvelle mythologie victimaire. Dans la pièce Big Shoot, il s'agit d'un affrontement entre un bourreau et une victime, alors qu'un homme accepte volontairement d'être mis à mort devant un public qui attend cette exécution avec impatience. Dans Blue-S-cat, une femme et un homme qui ne se connaissent pas sont tous les deux coincés dans un lieu clos. La femme se transforme en Ange exterminateur et finit par tuer celui qui est enfermé avec elle dans la cage d'ascenseur. Dans Misterioso-119, il s'agit d'une relecture du rituel catholique de la messe dans lequel une intervenante en théâtre, dans une prison pour femmes, sera tuée et mangée par les prisonnières, donnant une nouvelle signification à l'Eucharistie.

En analysant les pièces de Koffi Kwahulé, nous espérons être en mesure d'apporter une définition plus précise de sa vision kwahuléenne du monde, telle qu'elle est représentée à travers ses écrits. 


\section{CHAPITRE 1: «AFRICANITÉ» ET IDENTITÉ}

Avant de commencer notre analyse du théâtre de Koffi Kwahulé, il nous apparaît primordial de nous interroger sur la problématique que constitue la notion identitaire d'«africanité», étant donné que des auteurs «africains», comme Koffi Kwahulé, semblent remettre en question les rapports traditionnels entre les écrivains et leur pays d'origine par les nombreux déplacements géographiques qu'ils effectuent au fil des années. Koffi Kwahulé est né en Côte d'Ivoire, où il a étudié avant de compléter ses études en théâtre en France où la majorité de ses pièces sont publiées. La question qui se pose dorénavant est de savoir comment est-ce possible de définir un auteur africain? Nous avons donc passé en revue les études critiques et théoriques pour essayer de faire le point sur ce concept complexe et difficile à cerner puisque, de manière générale, ll sous-tend des liens avec l'Afrique, sans que ceux qui utilisent le mot définissent la nature de ces liens.

Nous devons d'abord insister sur le fait que la notion même d'«Afrique» demeure très problématique puisqu'elle est une invention coloniale. Avant les grandes périodes de colonisation, les «Africains» se définissaient d'abord eux-mêmes selon leur appartenance à un groupe ethnique, à une tribu ou à un royaume, avant de se voir imposer, par les colonisateurs occidentaux, l'étiquette d'«Africains». Rappelons que le continent «africain» est composé d'une multitude de groupes ethniques qui sont très différents les uns des autres. Ainsi, les «Noirs» constituent un ensemble qui est ethniquement mixte et qui renvoie aux Africains subsahariens, alors que les «Africains» comprennent également des populations d'origines berbère, arabe, maghrébine et kabile (notamment l'Algérie, la Tunisie, le Maroc) qui viennent du Nord. L'Afrique en tant que lieu géographique ne peut être considérée comme étant un ensemble homogène. 
Dans son livre L'Afrique fantôme, Michel Leiris ${ }^{7}$ traite justement de cette quête de l'Afrique lors d'un périple qui l'a mené de Dakar à Djibouti, entre 1931 et 1933. Il est intéressant de constater qu'après six mois de voyage, alors qu'il est arrivé au Dahomey (actuellement le Bénin), il écrit: «En route, le caractère exotique s'accentue. Les Tropiques exactement tels qu'on les imagine. Paysage déconcertant à force de ressembler à ce qu'on pouvait attendre» (140). Cette évocation de la nature met en évidence les a priori et les attentes qu'il s'est forgés au sujet du paysage africain et ce à quoi ce paysage devrait justement ressembler. Cependant, Leiris confond Tropiques et Afrique puisque ces deux espaces géographiques correspondent en fait à une même idée, celle des terres du sud, au sud de l'Europe, faut-il le préciser, celle de la chaleur intense et surtout celle où se retrouve le «Noir».

Il lui faudra poursuivre son voyage encore - jusqu'à l'approche de la frontière abyssine-avant d'affirmer: «Combien de kilomètres a-t-1l fallu que nous fassions pour nous sentir enfin au seuil de l'exotısme!» (226). Sa quête et son rêve d'exotisme ne seront pas là où il s'y attendait puisque cette Afrique mythique et légendaire n'existe pas. Il constate: «Voici enfin l'AFRIQUE, la terre des $50^{\circ}$ à l'ombre, des convois d'esclaves, des festins cannibales, des crânes vides. La haute silhouette du maudit famélique qui toujours m'a hanté se dresse entre le soleil et moi. C'est sous son ombre que je marche, ombre plus dure mais plus revigorante aussi que les plus diamantés des rayons» (225).

Il réalisera par la suite que l'Afrique qu'il cherche depuis son départ est une construction de l'imaginaire des Blancs européens, une construction de stéréotypes et de caractéristiques qui, inconsciemment, répondait à leurs besoins d'ailleurs et à leurs désirs d'exotisme. Avec $L^{\prime} A$ frique fantôme, Michel Leiris amorce la déconstruction de ce continent imaginaire, de cette Afrique fantomatique et «insaisissable» pour l'Européen, tout en prenant véritablement conscience de son statut d'étranger par rapport à l'Autre et également de son statut de colonisateur.

7 Mıchel Leırıs était écrivain, ethnologue, poète, critıque d'art et essayıste 
Edward Said a abouti à une analyse similaire à celle de Leiris avec son livre Orientalism, où il a démontré que l'Orient était une construction coloniale imaginée et fantasmée, notamment à travers le regard des auteurs et artistes britanniques et français, une construction qui n'avait donc rien à voir avec l'identité dite «orientale». Selon lui:

the imaginative examination of things Oriental was based more or less exclusively upon a sovereign Western consciousness out of whose unchallenged centrality an Oriental world emerged, first according to general ideas about who or what was an Oriental, then according to a detailed logic governed not simply by empirical reality but by a battery of desires, repressions, investments, and projections. (8)

À partir de ces exemples tirés des écrits de Leiris et Said, quel lien pouvons-nous tisser entre l'identité et la géographie? Il est important de rappeler que l'établissement, voire l'ımpositıon de ces frontières, souvent arbitraires, imaginées par les Occidentaux, n'était pas conçue selon les réalités sociales, géographiques, ethnologiques, historiques ou politiques de la période précédant la colonisation. Selon Benedict Anderson, dans son livre Imagined Communities, les frontières imposées par des pouvoirs coloniaux «shape the ımagination» (171) des Européens mais également des diverses communautés africaines et souveraines qui y vivalent déjà.

En effet, Anderson explique que la conception européenne par rapport à la cartographie visait essentiellement la mise en place du pouvoir et de l'autorité venus d'ailleurs. Les cartes, et surtout les frontières qu'elles délimitent, étaient donc des constructions purement artificielles et imaginaires qui visaient à légitimer un pouvoir économique.

Anderson va même plus loin en ajoutant que le fait d'asservir les populations grâce aux tracés des frontières correspond à ce qu'il appelle la «logoization» (176) géopolitique qui entraîne la création de cartes qui sont d'énormes casse-tête aux pièces interchangeables selon la couleur du colonisateur. Elles permettent d'établir des liens imaginés avec le pouvoir colonial. «In 
London's imperial maps, British colonies were usually pink-red, French purple-blue, Dutch

yellow-brown and so on. Dyed this way, each colony appeared like a detachable piece of a jigsaw puzzle. ... the logo-map penetrated deep into the popular imagination, forming a powerful emblem for the anticolonial nationalism being born (175).

Il nous faut donc tenir compte de toutes ces notions si nous voulons tenter de définir ce qu'est l'africanité. Comme nous l'avons vu, ce concept peut, dans un premier temps, être déterminé selon les origines et les lieux géographiques, c'est-à-dire par la création du continent africain. Nous avons vu que ce lieu n'est pas homogène puisque de nombreuses ethnicités s'y côtoient. Dans un second temps, nous devons également interroger tout ce qui a trait à la couleur de la peau. La peau noire peut-elle répondre directement à l'Afrique, au continent africain et à l'africanité afin de cerner et définir le «Noir»? Nous pouvons d'ailleurs ajouter que le lien qui unit le «Noir» et l'«Afrique» n'est pas aussi limpide que l'héritage colonial pourrait le laisser croire.

En effet, qu'est-ce qu'un «Noir»? Jean Genet a compris toute l'ambiguïté de cette question liée à la couleur de la peau lorsqu'un comédien lui demanda un jour d'écrire une pièce qui serait jouée par des acteurs «Noirs» pour un public «Blanc»». Dans Pour jouer Les Nègres, qui précède la pièce Les Nègres, Genet s'est interrogé ainsi: «Mais, qu'est-ce donc qu'un Noir? Et d'abord, c'est de quelle couleur?» Selon Genet, le «Noir» est une catégorie identitaire qui n'existe que par rapport au regard de celui qui se voit comme «non-Noir», dans le contexte d'une série infinie de jeux de représentations ou de mises en abîme qui finissent par annuler l'existence

8 La pıèce Les Nègres fut créée le 28 octobre 1959 par Roger Blın au théâtre de Lutèce à Parıs avec La troupe des Griots Il s'agıssait de treize acteurs noırs' Robert Liensol, Mamadou Condé, Gérard Lemoıne, Lydia Ewandé, Toto Bıssainthe, Darlıng Légitımus, Judıth Aucagos, Gısèle Baka, Dıa Fara, Edée Fortın, Georges Hılarıon, Théo Légıtımus, Bachır Touré Elle a eu une influence très importante sur les auteurs dramatıques antıllaıs des années 1970 qui ont vu, pour la première fors, des acteurs «Noırs» sur la scène parısıenne L'auteur martıniquass Danıel Boukman (Les négriers) s'inspıre de la mıse en questıon identıtaıre de Genet en alternant entre un passé esclavagıste et un présent marqué par une immıgration forcée vers la métropole Boukman révèle aussi les traces de Genet en empruntant son dispositıf scénıque d'orıgine piscatorıenne où ceux qui jouent les colonısateurs sont placés sur un échafaudage surélevés par rapport aux acteurs «Noırs» par terre, qui préparent le spectacle d'une mise à mort pour divertır les «Blancs». Vour Alvına Ruprecht et «Stratégıes d'une dramaturgıe polıtıque le théâtre antıcolonıal de Danıl Boukman " Annuaire théâtral 20 (2000). 59-72 
même de cette catégorie. Il s'agit évidemment du regard porté par le «Blanc» que Genet met en scène. La pièce Les Nègres se joue des stéréotypes, du racisme et des images que les «Blancs», les spectateurs scéniques et les spectateurs dans la salle, ont construit face aux «Noirs». Pour mieux déconstruire ces images, ${ }^{9}$ Genet a mis en abîme la cérémonie imaginaire du sacrifice d'une «Blanche» joué par des acteurs «Noirs» devant une Cour «Blanche», jouée également par des acteurs «Noirs» déguisés en «Blancs». Effectivement, cette Cour n’est pas vraiment «Blanche». Il s'agit de «Noirs» qui endossent les masques blancs pour jouer des figures du pouvoir «Blanc» sur des tréteaux surélevés. Ces figures observent le jeu qui se déroule plus bas sur la scène, soit des «Noirs» jouant les «Noirs» selon les images construites par les «Blancs». Genet affirme ainsi que le «Noir» signifie une identité insaisissable.

Le «Noir» constitue une catégorie identitaire liée à une perception de la couleur de la peau par le «non-Nour», pour signifier une différence dont la «couleur» est un signe visible, affirme Henry Louis Gates ${ }^{10}$ dans l'introduction de "Race," Writing and Difference: «But language is not only the medium of this often insidious tendency; it is its sign. Current language use signifies the difference between cultures and their possession of power, spelling out the distance between subordinate and superordinate, between bondsman and lord in the terms of their "race"» (6). Gates ajoute que «Noir» est d'abord un signe linguistique de dévalorisation construit par les Occidentaux afin de démontrer l'infériorité de l'Autre par l'entremise de sa couleur. «Noir» est donc la construction d'un signe linguistique qui signifie le «non-moi». «Race has become a trope of ultimate, irreductible difference between cultures, linguistic groups, or adherents of

9 Il faut préciser que Frantz Fanon (Peau noire, masques blancs) s'inspire du texte de Sartre, Réflexıons sur la question julve, quı dresse le portrait de l'antısémite et où 1 ll affirme l'importance du regard d'autruı quant à la prıse de conscience de so1 «Le Juıf est un homme que les autres hommes tiennent pour Juif voilà la vérité sımple d'où il faut partır c'est l'antısémite qui fait le Juif» (83-84) Sartre et Fanon s'inspirent de la pensée de Hegel pour quı "la conscience de so1 est forcément quelque chose qui se développe . dans le rapport à autru1» (Dionne), soit toujours dans un rapport intersubjectıf Dans son livre Peau noire, masques blancs, Fanon y ajoute la catégorie de race, ou le «Noir» se voit comme un «Non-Blanc», la négation du «Blanc», puisqu'll intériorise la manière dont les «Blancs» posent leur regard sur luı.

10 Henry Louıs Gates est dırecteur du W.E B Du Boıs Instıtute for Afrıcan and Afrıcan American Research à Harvard. 
specific belief systems which-more often than not-also have fundamentally opposed economic interests» (5).

En ce sens, les propos de Genet rejoignent directement ceux de Gates et également de Frantz Fanon, que nous préciserons un peu plus loin. Cependant, avant d'aborder les écrits de Fanon, nous croyons important de replacer dans le temps ce questionnement par rapport à la couleur de la peau. ${ }^{11}$ Nous nous attarderons donc au mouvement de la Négritude, tel qu'initié dans les années 1930, notamment par Aimé Césaire (Martinique), Léon Gontran Damas (Guyane française) et Léopold Sédar Senghor (Sénégal). À la base, la Négritude est un phénomène de résistance anticoloniale et antiraciste qui cherche à définir une essence «Noire» par opposition à une essence $« B$ Blanche».

Césaire tente de reconstituer un système de références culturelles africaines dans le contexte diasporıque. Il s'agit alors d'une nécessité stratégique pour lutter contre le racisme et pour revaloriser les origines afrıcaines qui avaient été mises à mal par la société coloniale française. La peau noire doit donc être un signe de ce qu'il faut revaloriser par la pensée de la Négritude. Cette prise de conscience afrocentrique s'est effectuée par rapport à la diaspora, où les héritiers de l'esclavage ont voulu marquer une résistance face au dominateur, en recréant des liens avec leurs racines africaines. Dans Culture et colonisation, Césaire souligne que la Négritude est une célébration de l'héritage africain et donc de cette culture à l'échelle

11 Il exıste un corpus critıque consıdérable sur l'identité afrıcaıne, le produit surtout des pensées afrıcaınes anglophones et notamment Ethiopia Unbound Studies in Race Emancipation, (1911) par Joseph Ephraim Casely Hayford Il s'agit du premier livre qui tratte de la question de "the African Personality» et fournit la clé du panafricanısme La rencontre où des Africains ont vraıment débattu de cette question d'Africanité pour la première fols a eu lieu dans la ville de Makerere (Ouganda) en 1962 Des écrivaıns africaıns, anglophones pour la plupart, ont posé ces mêmes questions Qu'est-ce que la littérature africaıne? Qu'est-ce un auteur africaın? (What is African Literature? What is an African writer?) Il faut faire la distinction entre cette rencontre de 1962, et celle sur le Monde Noır quı a eu lıeu à Parıs en 1956 Il s'agıssaıt alors de célébrer les réalısations du Monde «Noır»: écrıvaıns, artıstes, penseurs, maıs ceux-c1 n'ont pas vraıment abordé la question de l'Afrıcanıté. Il s'agıssait plutôt de repenser la Négritude et la sıgnification de la couleur de la peau. Les Africains anglophones par contre ont remis en question la langue anglaıse La question de l'Africanıté passatt donc par le débat sur la langue. Pensons surtout à l'essai d'Obi Walı intıtulé The Dead End of African Literature (1963) où 1 l écrit que les langues européennes ne peuvent être considérées comme faisant partie de la littérature africane parce qu'il s'agit de la langue du colonisateur Avec son essai Decolonising the Mind (1986), Ngugı Wa Thiongo a également beaucoup contribué à cette réflexion. 
continentale qui constitue l'«effort de toute collectivité humaine pour se doter de la richesse d'une personnalité» (191).

Selon Césaire, la colonisation a dépossédé les peuples de leur culture puisqu'elle a hiéarchisé les cultures européennes par rapport à la culture des pays colonisés. La Négritude s'inscrit dans ce débat qui vise à refuser l'acculturation imposée par l'idéal de la colonisation. Fanon tente de rendre transparente cette stratégie adoptée par les tenants de la Négritude qui ont recours aux notions des colonisateurs, afin de renverser leur discours raciste qui hiérarchise la culture. En effet, dans Les Damnés de la terre, Fanon écrit:

À l'affirmation inconditionnelle de la culture européenne a succédé l'affirmation inconditionnelle de la culture africaine. Dans l'ensemble les chantres de la négritude opposeront la vieille Europe à la jeune Afrique, la raison ennuyeuse à la poésie, la logique oppressive à la piaffante nature, d'un côté raideur, cérémonie, protocole, sceptıcisme, de l'autre ingénuité, pétulance. liberté, pourquoi pas luxuriance. Mais aussi irresponsabilité.(203)

Le «Noir» en tant que signe issu d'une construction européenne n'est pas différencié selon l'origine, la culture, la classe, la langue ou le groupe ethnique. Le «Noir» est un stéréotype uniquement défini par sa différence épidermique. Nous constatons que Césaire et Leiris ont proposé deux stratégies très différentes pour déconstruire le racisme même si ces idées ont été exprimées à la même époque. Selon Césaire, l'identité et la culture sont définies par la couleur de la peau noire—une réaction antithétique en réponse au racisme et à la domination—tandis que Leiris constate, pour sa part, que cette recherche d'identité et de culture «Noire» ne correspond pas aux fantaisies imaginées par l'Europe vis-à-vis de l'Afrique. Leiris offre une explication historique pour déconstruire le regard et les stéréotypes du colonisateur et des Européens. Césaire renverse plutôt le stéréotype du «Noir» proposé par le «Blanc», pour que la négation devienne une caractéristique positive. Césaire reprend ainsi les mêmes catégories stéréotypées du «Blanc» 
et du «Noir» dans une relation binaire identique mais il en renverse la hiérarchisation des valeurs, semblant insister sur l'importance du rêve, de la poésie et de l'irrationnel. Quoique troublantes, ces stratégies sont importantes, surtout à l'époque où le surréalisme prenait son envol. Chacun y puisait pour en extraire une matière nouvelle afin de transformer la société et l'homme, en rejetant le cartésianisme et la pensée rationnelle afin de libérer l'homme des systèmes de la pensée logique, issus des institutions politiques, des écoles, deș églises, des universités, et bien sûr, de la pensée coloniale et raciste. Il s'agissait de créer un nouvel ordre du monde. Dans l'essai Position politique du surréalisme, André Breton écrit:

C'est par l'affirmation de notre fidélité inébranlable aux puissances d'émancipation de l'esprit et de l'homme que tour à tour nous avons reconnues et que nous lutterons pour faire connaître comme telles.

«Transformer le monde», a dit Marx ; «changer la vie», a dit Rimbaud : ces deux mots d'ordre pour nous n'en font qu'un.» (95)

C'est dans cet esprit qu'il faut aborder le principe de la Négritude, bien que Frantz Fanon, tout comme Césaire, dénonce lui aussi le dominateur, car le «pays colonial est un pays raciste» (Fanon, Racisme et culture 128). Mais ce qui différencie les propos de Fanon de ceux de Césaire concernent l'identité et la culture, qui se constituent non pas selon la couleur de la peau noire, ni ces rapports binaires troubles qui essentialisent l'identité africaine: «ingénuité, pétulance, liberté, et pourquoi pas luxuriance» (Fanon, Damnés 203), mais plutôt par l'entremise du regard de l'Autre et qui appellent à la résistance. La pensée de Fanon, qui s'inspire de la lutte des classes selon une vision marxiste, est également construite par la nationalité, la culture, le genre et les lieux géographiques. Mais il fait la distinction entre la «culture africaine» et «la culture nationale» et, selon Fanon, les tenants de la Négritude font erreur quand ils parlent «davantage de culture africaine que de culture nationale» (Damnés 204). Fanon ajoute que l'Afrique n'est donc pas concevable sur le plan culturel puisque chaque pays possède sa propre culture. «La culture 
nègre, la culture négro-africaine se morcelait parce que les hommes qui se proposaient de l'incarner se rendaient compte que toute culture est d'abord nationale» (Damnés 20). Fanon insiste sur le fait que les problèmes des Africains sont très différents de ceux rencontrés par les Afro-Américains, tant du point de vue géographique et économique que social.

De plus, ce discours de Fanon rejoint celui de W. E. B. Du Bois dans The Souls of Black Folk sur la notion de double conscience, qui sous-tend la dualité, la contradiction, entre le fait d'être «Noir» et Américain, et qui détermine la conscience identitaire des Afro-Américains qui ont toujours l'impression de se voir à travers le regard de «l'Autre».,Du Bois décrit cette expérience vécue par le «Noir» dans «a world which yields him no true self-consciousness, but only lets him see himself through the revelation of the other world» (8).

Nous pouvons avancer que le caractère «africain» est, en ce sens, une invention occidentale qui permet de rassembler sous une même appellation tout ce qui est d'origine et de culture du continent africain. En effet, les marques stéréotypées qui sont acceptées selon les références traditionnelles par la doxa européenne sont généralement associées, par exemple, à l'Afrique du conte oral, de la musique et de la danse traditionnelles, aux lieux «spécıfiquement afrıcains» et aux éléments concrets tels que les vêtements, les habitudes de vie, l'alimentation, les traditions, les comportements en société. Il s'agit de renvois à des références construites par des non-Africains et qui sont des stéréotypes explicitement reconnaissables par ces mêmes nonAfricains.

En ce sens, le concept même d'africanité semble se transformer selon les diverses analyses qui en sont faites, puisqu'il faut tenir compte des débats géographiques, ethniques, culturels, idéologiques et même techniques qui ont cours aujourd'hui.

Par exemple, l'anthropologue Jacques Maquet, dans son livre Africanité traditionnelle et moderne, parle d'une africanité qui est à la fois diversité et unité culturelles liée à un héritage commun des sociétés et qui est inspirée des connaissances, des comportements, des phénomènes 
et des idées partagées par tous les Africains: «L'africanité est aussi un outil conceptuel qui permet d'appréhender ce qui est commun aux différentes civilisations africaines. Très proche du concept de Négritude, il s'en différencie par son orientation. Alors que la Négritude avait pour fonction essentielle d'affirmer une personnalité culturelle jusque là aliénée, l'africanité vise à la compréhension, à l'analyse» (15).

Jacques Maquet identifie trois sources de l'africanité traditionnelle qu'il regroupe ainsi: identité des techniques de subsistance, diffusion culturelle en Afrique subsaharienne et isolement de l'intérieur du continent. Avec l'insertion dans le monde à l'époque industrielle, il ajoute un quatrième élément à cette liste qui tend vers une africanité moderne. Il définit l'africanité comme étant «l'ensemble des traits culturels communs aux centaines de sociétés d'Afrique subsaharienne» (53).

Il nous semble que le discours de Maquet, malgré une véritable volonté d'expliquer l'Afrique, soit empreint d'une certaine perception colonialiste selon laquelle les sociétés africaines répondraient désormais à des attentes et classements typiquement européens, à travers lesquels les Européens peuvent se tracer un chemin qu'ils reconnaissent. Ce genre de discours fait dire à Sylvie Chalaye que:

l'africanité au sens culturel où l'entendent les Européens, est une notion inopérante parce qu'entachée d'une velléité simplificatrice et unificatrice qui remonte à une posture colonialiste. L'Afrique est loin d'être une unité culturelle et historique. Mosaïque de peuples et d'ethnies disséminés par la traite, fractionnés par le partage colonial de l'Europe, elle ne peut engendrer une valeur identitaire unificatrice sans totalitarisme. (Briser l'enclos 32)

Puisque nous savons que la couleur de la peau n'est pas signe d'une homogénéité culturelle mais plutôt de «différence» selon Gates, l'esprit de la Négritude ne peut pas répondre directement à cette idée d'africanité. Toutefois, il importe de préciser que Léopold Sédar Senghor 
définit l'africanité dans Les fondements de l'Africanité, Négritude ou Arabité comme étant un ensemble de valeurs «qui soient communes à tous les Africains et qui soient, en même temps, permanentes» (10). Il considère qu'il s'agit d'une «symbiose complémentaire des valeurs de 1'Arabisme ${ }^{12}$ et des valeurs de la Négritude» (10), qui repose essentiellement sur un métissage des races et des ethnies et sur des convergences entre les cultures arabe et négro-africaine.

En matière de création artistique et culturelle, de jeunes dramaturges d'origine africaine se réclament aujourd'hui de cette conscience du métissage. C'est le cas du dramaturge Koulsy Lamko, originaire du Tchad, qui s'identifie comme étant issu du mouvement de métissage de l'africanité et non plus de celui de la Négritude. Il affirme que les mouvements négritudiens ont été nécessaires à une certaine époque de revendication mais qu'ils sont aujourd'hui dépassés, étant donné que les frontières culturelles et géographiques n'existent plus comme auparavant et que l'identıté devient ainsi individuelle et non plus collective. Il indique en effet que «La Négritude dans son contexte avait sa raison d'être.... Mais on ne peut nier l'évolution du monde et les phénomènes dialectiques qui lui sont inhérents. C'est estimer qu'en Afrique rien ne change» (Chalaye, Africanité vagabonde 27). Nous voyons que l'Africanité est un concept identitaire qui est en transformation permanente.

En partant de la Négritude, de nouvelles notions ont émergé pour redéfinir de nouvelles dynamiques identitaires, sociales et culturelles qui soient métissées. Les métaphores botaniques de la racine et du rhizome illustrent ces transformations. L'identité-racine de la Négritude est donc unique par opposition à l'identité-relation ou multiple, également appelée identité rhizomatique.

Deleuze et Guattari ont été les premiers à employer ces métaphores dans leur livre Rhizome: Introduction. «Un rhizome comme tige souterraine se distingue absolument des racines et radicelles. Les bulbes, les tubercules sont des rhizomes» (17). Le principe de connexion qu'ils 
ont élaboré signifie que «n'importe quel point d'un rhizome peut être connecté avec n'importe quel autre, et doit l'être» (18).

En s'inspirant justement de Deleuze et Guattari et de leur notion de rhizome, Édouard Glissant a lui aussi employé ces images botaniques pour traiter de la question du métissage dans Identité comme racine, Identité comme relation. D’un point de vue identitaire, «La racine est unique, c'est une souche qui prend tout sur elle et tue alentour» (202). Elle n'admet aucune rencontre qui puisse engendrer quelque chose de nouveau. En opposition à cette linéarité identitaire, Glissant parle d'une identité-relation qui est «une expérience des contacts entre cultures» (202). C'est pourquoi il explique: «La terre antillaise n'est pas un territoire, c'est une terre rhizomée, où se fonde un rapport nouveau» (202).

La métaphore du rhizome est une image qui permet aux écrivains de passer de la notion de la Négritude à la pensée postmoderne du multiple et de l'instable, une visıon du monde qui refuse la structure binaire de la pensée de la Négritude. Cependant, ces ımages de rhizomes et de métissage sont des métaphores inspirées de la botanique. Il ne s'agit pas de concepts qui soient vraiment opératoires dans le contexte de la pensée littéraire, mais plutôt des références à une catégorie scientifique à partir de laquelle les artistes peuvent créer des textes, développer des images et ouvrir un monde de l'imaginaire. Ceci afin de mieux cerner et faire comprendre les transformations identitaires complexes, désignées désormais comme métissage et qui sont en train d'avoir lieu dans tous les domaines de la vie humaine. Ils représentent une certaine manière de percevoir le monde qui montre que l'identité unique est désormais difficile, voir impossible à concevoir.

C'est à travers cette notion de métissage qu'il faut lire les propos du dramaturge congolais Caya Makhélé au sujet de sa part d'africanité, qui est, selon lui, la somme de ses expériences et qui est donc nécessairement en perpétuelle construction:

Mon africanité à moi est différente de celle d'Amadou Hampaté Ba, de celle 
d'Ahmadou Kourouma ou encore de celle de Koffi Kwahulé et pourtant ils ont une part d'africanité qui peut être semblable à la mienne, mais la mienne est particulière parce que j'ai vécu des choses particulières qu'ils n'ont pas dû vivre, et qu'ils ont vécu eux aussi d'autres expériences que les miennes. Je ne m'insère pas dans un destin collectif, je m'insère dans un destin individuel qui peut être un destin collectif. (Chalaye, Afrique noire et son théâtre 50)

Le débat sur le concept «d'africanité» démontre que les catégories identitaires se transforment, se renouvellent et se redéfinissent sans cesse. Ainsi, certains auteurs d'origine africaine représentent justement cette instabilité. Ils renouvellent les critères et les catégories de l'africanité à travers l'évolution de leurs œuvres théâtrales. Nous voyons que, selon Sylvie Chalaye, l'africanité est d'abord un «mythe de la conscience occidentale, mais qu'il existe aujourd'huı une africanité autre, une africanité quı s'invente dans la créatıon contemporaıne et qui n'est pas où on l'attend» (Masques 5). C'est notamment le cas de Koffí Kwahulé quı contribue à la transformation de l'africanité par sa manière de repenser les catégories du théâtre. Par exemple, nous pouvons nous demander si son œuvre représente un théâtre traditionnel et ritualisé où la présence des griots et chanteurs s'impose. S'agit-il d'un théâtre qui met en scène des personnages «Noirs» et «africains»? Et si ce n'est pas le cas, cela signifierait-il que le théâtre «africain» a été «contaminé» et perd donc sa valeur africaine? Que signifient les affirmations de certains critiques qui disent: «Du coup on a tendance à entendre: "Mais de toutes les façons ce sont des Africains dénaturés, un peu blanchis et qui ont presque renié quelque part ce qui appartient à leur particularité"» (Chalaye, Africanité et création 8). Sylvie Chalaye critique cette façon plus traditionnelle d'aborder la question identitaire africaine au profit d'une approche plus contemporaine.

En fait, deux visions s'opposent autour de ce débat. Il y a celle qui repose sur une notion traditionnelle claire d'une identité africaine qui porte en elle les marques d'une authenticité 
culturelle en lien avec la Négritude ou l'afrocentrisme, pensées développées en relation binaire, et il y a celle prônée par Fanon où les frontières entre les différentes identités nationales sont clairement définies. Il y a ensuite la nouvelle façon d'aborder ce questionnement, comme le font les dramaturges d'origine africaine qui vivent aujourd'hui en Europe et qui rejettent les préoccupations de Fanon. Sylvie Chalaye estime qu'un «nouveau» théâtre africain émerge d'une population de dramaturges plus nomades, alors que ceux-ci remettent en question les marques traditionnelles de la culture «africaine».

\subsection{Les dramaturges de la «nouvelle africanité»}

Nous pouvons nous questionner sur la place du dramaturge Koffi Kwahulé dans le contexte actuel de la création. Originaire de la Côte d'Ivoire, Koffi Kwahulé vit et travaille en France depuis une trentaine d'années. Dans ses pièces, il explore la nature des rapports humains, rapports souvent conflictuels, marqués par la sexualité, la violence et la mort. Les rapports de domination et de soumission deviennent des thèmes récurrents de son œuvre et sont intimement liés à la question du racisme et de l'ımmigration, qu'il explore à partir de différentes perspectives: dominateur-dominé, homme-femme, étranger-terre d'accueil, prisonnier-liberté.

Depuis 1977 , il a écrit plus de 30 pièces qui ont presque toutes été jouées. Il est également comédien, metteur en scène et romancier. Certaines de ses pièces ont été traduites et mises en scène aux États-Unıs, en Angleterre, en Allemagne et en Italie notamment. Il a fait ses études à l'Institut national des Arts d'Abidjan, à l'École de théâtre de la Rue Blanche, à Paris, puis à la Sorbonne Nouvelle, où il a obtenu un doctorat d'études théâtrales.

Son œuvre théâtrale exemplifie le débat concernant la définition de l'africanité et sa représentation dans la production artistique actuelle. L'expression de la conscience identitaire du dramaturge s'est transformée depuis ses débuts d'écrivain où les personnages étaient tous «Noirs». Dans sa toute première pièce, Le Grand-Serpent (1977, inédit, archives de l'auteur), nous pouvons remarquer la présence de quelques éléments qui pouvaient démontrer une certaine 
vision plus traditionnelle de la pratique théâtrale. En effet, l'action du premier tableau se déroule sur la place du village, où un conteur raconte justement l'histoire de ce village et de la vie de Haïka, un enfant qui s'est transformé en monstre et de la manière dont il est devenu le GrandSerpent, chef sanguinaire du village, à l'image des dictateurs africains. Les personnages présents sur scène chantent un chant triste et sont accompagnés à la cora. La pièce est en fait une légende africaine contemporaine puisqu'elle traite de questions qui ont directement touché un certain nombre de pays du continent.

Dans son livre Pour une critique du théâtre ivoirien, Koffi Kwahulé, retrace justement ses débuts au théâtre. Le Grand-Serpent «s'appuie sur un récit, et qui plus est, un conte avec des personnages-masques. D'autre part, la danse est ici nettement en retrait: un seul personnage (à la représentation) danse au Tableau I (le Devin)» (254).

À cette époque, l'auteur, qui était étudiant en théâtre, rappelle qu'il fait partie du groupe «Les Étudiants Ivoiriens en art dramatique à Parıs» (250), qui retourne en Côte d'Ivoire pendant les vacances pour y présenter du théâtre. L'approche de ces jeunes sans moyens déconcertait souvent les spectateurs, «mais la pauvreté de leur théâtre résultait plutôt d'un double choix esthétique et idéologique» (251).

En 1981, la pièce Le Grand-Serpent est censurée par les autorités après une seule représentation. De plus, «Les efforts de distanciation du metteur en scène Guédéba furent par ailleurs perçus comme des maladresses» (254).

À l'été 1982, «Les Étudiants» retournent de nouveau en Côte d'Ivoire pour y présenter quelques pièces dont $1+l=1$ de Koffi Kwahulé, dans une mise en scène de Guédéba Martin. $l+l=1$ (1982, inédit, archives de l'auteur) relate la rencontre de deux personnages autrefois amis. Pendant leurs études en France, ils projetaient de changer l'ordre politique de leur pays dès leur retour chez eux. Mais l'un est devenu un intellectuel rangé bien nanti, tandis que le second sort de prison après avoir purgé une peine pour divergence politique. Ce dernier veut se venger de son 
ancien ami aujourd'hui nanti, qui a laissé tomber leur engagement politique, mais aussi parce qu'il lui a volé sa femme et son enfant pendant qu'il était en prison. Le prologue est une cérémonie qui se déroule vraiment à l'extérieur du théâtre, un acte rituel initiatique qui est vécu par les spectateurs et où prennent part le guide et le banni. Ensuite, les spectateurs doivent retourner à l'intérieur de la salle dans laquelle ils retrouvent le guide et le banni sur scène, où ils incarnent dorénavant le nanti et l'ancien prisonnier.

Hormis le guide de l'initiation, la pièce ne comporte pas d'autres éléments pouvant être associés à une certaine tradition orale africaine comme dans Le Grand-Serpent. En fait, nous constatons que la pratique d'écriture de Koffi Kwahulé révèle, avec $1+1=1$, une nouvelle perspective diasporique quant à la manière de percevoir les problèmes de son pays. De plus, la pièce a été, en Côte d'Ivoire, la toute première à deux personnages. «Je n'avais jamais écrit de pièces pour deux comédiens car, en Afrique noire, un dialogue à deux n'existait pas pour la scène; on considérait cela comme un truc de Blancs» (Kwahulé, entretıen inédit).

Même si cela a déconcerté le public, la pièce a connu, malgré tout, un important succès et a été enregistrée par la télévision. «Le succès de $l+l=1$ est aussi brutal qu'inespéré, car malgré l'échec des tentatives brechtiennes du Grand-Serpent, Guédéba et Kwahulé n'ont pas abandonné l'idée d'un théâtre qui casse avec le théâtre préexistant» (Kwahulé, Critique du théâtre 256).

Toutefois, si Kwahulé reconnaît le caractère inédit de $1+1=1$ par rapport à ce qui est habituellement attendu du théâtre ivoirien, il considère que ce qu'il proposait ressemblait davantage à une pièce de théâtre française:

Enfin $1+1=1$, la pièce de toutes les innovations! Considérer que «les deux seuls personnages»), la déambulation ${ }^{13}$ et l'absence de salut final sont des innovations, c'est implicitement souligner l'extrême pauvreté imaginative du théâtre ivoirien. Ce

13 La déambulation est le fait, au théâtre ou dans les arts de la rue, de voir les comédıens se déplacer d'un lieu à l'autre pendant la représentation ou le spectacle 
dialogue où un personnage accuse un autre de lui avoir volé sa femme et son enfant, ce dialogue qui n'est pas dénué d'un certain suspense a de forts accents de drame bourgeois français. (258)

Nous voyons que très tôt dans son écriture dramaturgique, Koffi Kwahulé a transgressé une certaine tradition africaine qui l'a rapproché d'un théâtre français plus contemporain. Dans $L e$ Grand-Serpent, cette transgression était davantage révélée dans la mise en scène et ses effets de distanciation. Dans $1+1=1$, elle était contenue dans l'ensemble du texte et dans la mise en scène. Seul le prologue était inspiré d'un certain rituel initiatique pouvant être associé à la tradition.

Dans ses pièces suivantes, Koffi Kwahulé indique avec précision la couleur de la peau de ses personnages, notamment dans Cette vieille magie noire et Bintou, que nous étudierons au chapitre suivant consacré aux mythes. L'action de ces deux pièces ne se déroule toutefois plus en Afrique mais plutôt à New York pour la première, et en France pour la seconde. Au fil des pièces, le dramaturge a donc proposé de nouvelles catégories du théâtre «africain», bien que, par la suite, toutes indications quant à la couleur de la peau ou au lieu d'origine aient complètement disparu de son œuvre.

Le dramaturge Koulsy Lamko est un autre exemple de cette mouvance vers le renouvellement de la manière de représenter l'Afrique au théâtre. Il résume ainsi sa vision qui s'applique tant à son travail qu'à celui de Koffi Kwahulé ou à d'autres dramaturges contemporains: «Et l'identité africaine aujourd'hui c'est cette errance, cette quête, ce questionnement même de l'identité. Il faut accepter l'idée qu'il y a des théâtres africains en mutation qui appartiennent d'abord aux individus qui les font et non pas à un théâtre "africain" ou "noir"» (Chalaye, Africanité vagabonde 27).

De nombreux dramaturges d'Afrique de l'Ouest ont choisi de vivre et de travailler en 
France, alors que d'autres comme Koulsy Lamko ${ }^{14}$ et José Pliya ${ }^{15}$ parcourent le monde. Cette nouvelle dramaturgie du nomadisme va au-delà des frontières africaines, étant donné que ces auteurs vivent désormais à l'extérieur de leur pays d'origine. Comme le fait remarquer Sylvie Chalaye, ce n'est pas que l'Afrique soit «absente de leur théâtre, ce sont eux qui sont absents à une certaine idée que l'on souhaiterait qu'ils offrent de l'Afrique» (Briser l'enclos 33).

Selon Ndongo M'Baye, ${ }^{16}$ «l'africanité», comme tous les concepts du genre-européanité, américanité, asiaticité, océanité, et nous pourrions ajouter celui de québécité — ne veulent rien dire parce qu'ils sont trop vagues, «tant dans une dimension et une perspective historique, sociologique, économique que culturelle et politique» (36). Il questionne également ce qu'il appelle une «commodité lexicale dont la signification chercherait en vain son sens» (36). Pierre Laurette apporte un éclairage linguistique quant à la signification de ces concepts: «Le suffixe ité implique que l'acte descriptif, sémantico-référentiel et définitoire est sans fin; ité ımplique une configuration symbolique, imaginaire de nature non exhaustive» (26).

Par ailleurs, M'Baye s'interroge sur les liens troubles et les regards croisés qui ont été échangés entre Africains et Européens dans une perspective historique. Il dit y voir «une relation contre nature, entre le regard européen chargé de clichés et de poncifs caractérisant "l'Africain" et le regard apaisant et conforté de l'Africain prêt à rendre aussi fidèle que possible cette image que l'Autre a de lui» (36).

M'Baye se demande également si l'écriture ne serait pas un geste, «une activité dénuée "d'africanité"» (37). Il affirme que, compte tenu des difficultés liées à sa diffusion, cela démontrerait la place prépondérante des traditions orales africaines au détriment de la nouvelle

14 Koulsy Lamko est né au Tchad, pour ensuite s'installer au Burkına Faso puis au Rwanda Il est notamment l'auteur de Ndo kela ou l'inttiation avoriée, Tous bas si bas, Regard dans une larme

15 José Plıya est né au Bénın avant de vivre en France, au Cameroun, en Dominique et en Guadeloupe où 1 dirıge actuellement l'Artchıpel, Scène Natıonale à Basse-Terre. Il est notamment l'auteur de Konda le requin, Concours de circonstances, Le Masque de Stka, Le Complexe de Thénardier et Nous étıons assis sur le rivage du monde 16 Ndongo M'Baye est docteur en lettres, sociologue, professeur associé à l'Unıversıté Cheıkh Anta Diop de Dakar (Sénégal), journalıste, écrivain et poète. 
production écrite. «Dans leur essence, ces traditions sont incompatibles avec l'écriture et montrent s'il en était besoin, que l'Afrique n'est pas le continent de l'oralité pour rien» (37). Il semble donc que, pour lui, «l'africanité» demeure un concept associé à la conservation des traditions spécifiquement africaines, traditions qui émergent de l'oralité. Nous pouvons certainement remettre en question cette vision des choses qui est à l'opposé de la métaphore des identités rhizomatiques telle que formulée par Glissant. Ces opinions de M'Baye semblent contredire la grande majorité des auteurs d'origine africaine qui vivent en Europe, là où leurs œuvres sont écrites et publiées. Ces auteurs constituent même un nouveau mouvement de la littérature afro-européenne, dont Koffi Kwahulé est l'une des figures les plus importantes. À la différence de cette vision d'une Afrique construite par une culture et une société de l'oralité et qui semble remettre en question l'écriture, il faudrait examiner les pratiques qui insistent sur une vision plus instable des catégories culturelles habituelles. Le sociologue d'origine camerounaise Francis B. Nyamnjoh s'intéresse particulièrement aux questions des médias et de la démocratie, ainsi qu'à la xénophobie et à la citoyenneté en Afrique du Sud. Il définit l'africanité comme étant «a process, not something one acquires from birth, transmitted through the "life essence of a Black african father", and to be protected from contamination by other ways of seeing and doing. Being African is not a birthmark. ... it would be quite misleading to assume from this a counter-notion of a universal and homogeneous African cultural identity» (27).

Quelles soient africaines ou ayant trait à tout autre groupe ethnique ou racial, les identités, sont donc en perpétuel mouvement, car elles sont soumises aux influences extérieures. Ces rencontres, qu'elles soient harmonieuses ou qu'elles produisent de vives secousses, s'opposent toujours entre elles et sont sujettes à de constantes redéfinitions. Selon Nyamnjoh, l'africanité n'est pas une «essence» mais bien une construction qui s'opère au fil du temps. Il affirme que «identities are therefore not constants, but subject to renegotiation with new experiences and 
aspirations» (27).

Nous pouvons observer que cette approche se distancie de manière importante du mouvement de la Négritude où la couleur de la peau était l'élément central. De plus en plus présente aujourd'hui, cette philosophie met entre parenthèses la notion même de Négritude puisque le nouveau concept d'identité africaine, qui émerge actuellement, montre qu'il n'a rien à voir avec la couleur de la peau. L'africanité est donc en constante transformation et doit être interprétée à travers les expériences de chacun.

Stuart Hall, lui-même d'origine jamaïcaine, estime en effet que l'identité n'est pas quelque chose «innate in consciousness at birth» mais bien «something formed through unconscious process over time» (122). Hall ajoute qu'il ne faut pas considérer l'identité dans une perspective devant aboutir à quelque finalité que ce soit, mais plutôt adopter la notion d'identification qui est, pour sa part, toujours en train de se développer, de se formuler et de se transformer.

Les propos de Hall rejoignent ceux de Kwame Anthony Appiah dans le livre In my Father's House, au sujet de la construction des identités africaines:

First, that identities are complex and multiple and grow out of a history of changing responses to economic, political, and cultural forces, almost always in opposition to other identities. Second, that they flourish despite what I earlier called our "misrecognition" of their origins; despite, that is, their roots in myths and lies. And third, that there is, in consequence, no large place for reason in the construction--as opposed to the study and the management- of identities. (178)

Si Stuart Hall s'intéresse à une identité africaine individuelle en devenir, Francis B. Nyamnjoh indique que l'identité africaine, soumise à de nombreuses influences, doit tenir compte de ses expériences passées - donc de ses expériences collectives—pour être également capable de se définir. Ce dernier cite en exemple les échanges entre les cultures et notamment ceux qui ont 
eu lieu dans le contexte de l'esclavage et du colonialisme. Et bien que la culture de l'ethnie dominée se soit souvent heurtée à celle du dominateur, les échanges ont toutefois eu lieu et ceuxci doivent être pris en compte. Ces expériences passées ne peuvent pas être ignorées quand il s'agit de construire une identité africaine, car ayant été assimilées, elles font obligatoirement partie de l'expérience et de l'héritage individuel et collectif. Toujours selon Francis B. Nyamnjoh, les déplacements géographiques des populations et des individus, tout comme la multiplicité des origines africaines, doivent aussi faire partie de cette grande réflexion: «We must indeed be creative, negotiating, dynamic and realistic in our claims about entitlements as Africans» (28).

\section{2 «Africanité» et langue}

Selon Hall et Nyamnjoh, la culture africaine qui sculpte l'identité africaine n'est pas un phénomène qui se déploie en vase clos, puisqu'elle continue d'être soumise à des influences multiples et extérieures depuis longtemps. Dans le cadre de ces échanges, qui ont souvent été forcés par le pouvoir colonial, la question de la langue est cruciale. Le continent africain est aujourd'hui formé par des pays dont l'une ou l'autre des langues dites officielles est le français, l'anglais, ou le portugais, un signe toujours tangible de l'ancienne présence coloniale. Puisque la construction de l'identité africaine comprend ces échanges forcés, «l'africanité» pourrait-elle être déterminée en partie par la langue du colonisateur? Son appropriation constitue-t-elle un autre aspect de la notion «d'africanité» et d'identité africaine? Que dire des auteurs, poètes et dramaturges qui se sont mis à écrire dans la langue du colonisateur?

Jean-Paul Sartre dans Orphée noir, préface de l'Anthologie de la nouvelle poésie nègre et malgache de langue française de Léopold Sédar Senghor, aborde cette question, à savoir si l'identité africaine ne perd-elle pas une part d'elle-même en se pliant à la langue européenne. N'est-elle pas condamnée à être perdue ou oubliée quand elle se cherche ailleurs, dans une langue non-africaine? Sartre rejette cette idée puisque, selon lui, «chacun sait que ce sentiment d'échec 
devant la langue considérée comme moyen d'expression directe est à l'origine de toute expérience poétique» (xix).

Selon Souleymane Bachir Diagne, ${ }^{17}$ cette affirmation de Sartre, au sujet de la littérature africaine dans la préface de l'Anthologie de la nouvelle poésie nègre et malgache de langue française, démontre que «the single voice of peoples ... constituted one single refusal and one single affirmation, namely ... Africanity which Aimé Césaire had coined as Negritude» (20). En effet, le mouvement de la Négritude a permis de rassembler sous une même bannière plusieurs voix et d'affirmer la légitimité des origines de ces êtres humains qui pouvaient enfin parler d'une seule et même voix. À cette époque, le recours à la langue française était nécessaire afin de regrouper les voix et de lutter contre le racisme et le colonialisme.

D’après Souleymane Bachir Diagne, le recours à la langue française, par lequel est portée l'africantté, ne constitue pas une contradiction en soi. «This is Sartre's thesis: of the thinking apparatus of the enemy there has indeed been appropriation and the foreign language is not foreign anymore» (20). Selon nous, cette appropriation du verbe ne constitue ni un retour en arrière ni une volonté d'assimilation.

En se référant à la préface de Sartre, Souleymane Bachir Diagne répond donc à la fois à Fanon et à M'Baye. En effet, le recours au français, comme nous l'avons vu, a permis de rassembler des voix pour n'en faire qu'une seule malgré le fait qu'il s'agisse de la langue du colonisateur et qu'elle en contienne des traces anciennes. Bachir Diagne répond à Fanon au sujet de la diversité culturelle — lui qui prônait l'unité nationale—et à M'Baye au sujet du fait que l'écriture serait un geste anti-africain.

Souleymane Bachir Diagne explique que l'usage de la langue française ne constitue donc pas une trahison. Grâce à une langue commune, il a été possible de donner une légitimité aux peuples d'origine africaine qui ont pu s'affirmer au sein de la pensée de la Négritude. À l'époque, 
au début de la Négritude, cette pensée plus radicale était nécessaire afin de lutter contre le racisme. Compte tenu de la situation culturelle et politique spécifique au cadre de la Négritude, nous pouvons ajouter qu'il fallait alors conscientiser le public d'où a émergé une vision plus afrocentrique de la lutte des Noirs. Nous voyons que les rapports à la langue française ont beaucoup changé, notamment en ce qui a trait aux auteurs contemporains d'origine africaine qui ont choisi de travailler en France et, par le fait même, d'écrire et d'être publié en français.

Il est pourtant difficile d'ignorer le fait que certains auteurs délaissent le français ou l'anglais afin de privilégier leur langue maternelle. C'est le cas de Ngugi wa Thiongo, ${ }^{18}$ qui a choisi la langue des villageois pour son théâtre et ses œuvres littéraires de fiction, non pas en reniant la langue anglaise mais plutôt en considérant, selon Souleymane Bachir Diagne, «what authentic self-writing means» (22). Ainsi, en passant de l'anglais au gikuyu ${ }^{19}$ «he had transformed the literary act into sociocultural and, ultimately, political action» (23). Ngugi wa Thıongo, tout comme Cheikh Aliou Ndao ${ }^{20}$ et Boubacar Boris Diop, ${ }^{21}$ a choisi de faire de même afin de pouvoir entrer plus facilement en relation avec ses lecteurs, «in order to perform Africanity» (Bachir Diagne 23). Selon ces auteurs, la littérature africaine ne peut seulement atteindre son sens ultime, tant du point de vue esthétique que politique, qu'en émergeant d'une langue africaine qui n'aura pas subi l'influence du colonisateur.

Toutefois, en réponse à l'approche des auteurs qui ont effectué un retour à leur langue maternelle, Souleymane Bachir Diagne estime qu'il ne faut pas poser ces questions comme s'il s'agissait d'un choix mais plutôt comme une possibilité de choisir. Selon lui, la question de la langue doit demeurer ouverte et sans réponse fixe, car "somehow Africanity is this possibility of

18 Ngug1 wa Thıongo est orıgınaire du Kenya. Il est professeur d'anglaıs et de littérature comparée à la Unıversity of Calıforna, Irvine Il a choısı de délaısser la langue anglasse pour écrire en gikuyu, sa langue maternelle

19 La langue gıkuyu peut aussı s'écrıre kıkuyu

20. Le poète et dramaturge sénégalaıs Cheıkh Alıou Ndao a traduit ses æuvres en wolof. Il estıme que les auteurs africains devraient faire comme luı afin d'intéresser les gens aux langues natıonales

21. Boubacar Borıs Diop est écrivaın, journalıste et dramaturge Il a publié Doomı golo, son premier roman en wolof, en 2003 En 2009, 1 publiat Les pettts de la guenon, traduction françaıse de Doomı golo 
coming and going from one language to another» (23).

Francis B. Nyamnjoh précise un peu plus cette idée en affirmant que cette rencontre entre l'Afrique et l'Occident a provoqué la naissance d'une langue nouvelle, en réussissant une forme de domestication et de recréation qui unit les langues de l'Occident et celles de l'Afrique: «The outcome of this process is certainly a notion of identity in Africa as hybridity or metissage, which, though drawing heavily from mainstream cultures and values, cannot be reduced to these without losing some of the richness of the continent's experience in the encounters of cultures»(28).

Cependant, la notion d'identité, en dehors de la question linguistique, demeure un sujet sensible au sein même des auteurs et critiques africains, surtout lorsque des créateurs africains revendiquent haut et fort leur identité de créateur et de citoyen du monde, du fait qu'ils vivent à l'extérieur de l'Afrique. Cette nouvelle façon de voir le monde irrite Bouboucar Boris Diop qui, de son côté, prône une certaine forme de résistance de la part des créateurs africains, surtout dans le contexte actuel de la mondialisation. Ce dernier ne comprend pas cette nouvelle génération qui refuse l'ancienne définition monolithique de l'étiquette afrıcaine et qui se définit comme citoyens du monde, «pour ne plus être prisonniers de leur négritude» et préfère «recracher leurs racines comme un fruit amer» (45).

Selon Diop, ces jeunes créateurs ne sont pas très loin de renier leurs origines, alors que des intellectuels se sont battus pendant des années pour justement faire accepter une nouvelle identité nègre au reste du monde, à la suite de l'esclavage et de la colonisation. Il dénonce le fait que plusieurs auteurs africains aient quitté l'Afrique et qu'ils continuent, malgré tout, de parler de l'Afrique «dont ils ne savent plus rien» (48). Ils deviennent, toujours selon Diop, les seuls dépositaires d'une certaine parole à être écoutée en dehors de l'Afrique et à l'intérieur du continent.

Mais, nous pourrions dire que tout ce que ces auteurs réclament, c'est simplement le droit 
d'être reconnu en tant qu'écrivain et non pas en tant qu'écrivain africain. Cette nuance nous semble primordiale car l'identité de ces auteurs est d'abord définie par l'écriture.

En tenant compte de toutes ces orientations, nous constatons que «l'africanité» est un concept en mouvement et en pleine évolution, constitué de catégories qui se transforment constamment avec les conditions de possibilités du savoir de chaque époque et de chaque individu. En fait, il s'agit d'une notion qui est très controversée et d'un sujet de discussion qui préoccupe beaucoup les écrivains qui ont quitté leur pays natal sur le continent africain.

Nous avons vu que le concept «d'africanité» comporte une réflexion sur la couleur de la peau «noire». Récapitulons cette réflexion en affirmant que le «Noir» est une catégorie identitaire qui ne peut exister que dans son rapport avec le «non-Noir», selon Fanon. Dans ce contexte, «Noir» est un manque, car il signifie «non-Blanc». En revanche pour Gates, «Noir» est plutôt un signe. Cette vision de la couleur est aussi rattachée à la notion géographique du continent africain imaginé et fantasmé par le colonisateur et l'étranger. Le pouvoir colonial a ensuite imposé ses règles, ses frontières et surtout son pouvoir économique.

Nous avons également rappelé que la signification de «Noir» s'est transformée. Il s'agissait alors d'un signe qui renvoyait à l'Afrique et à la colonisation. Par la suite, le «Noir» est devenu une essence Noire avec le mouvement de la Négritude qui a émergé pour résister au pouvoir colonial et à sa vision raciste, déplaçant ainsi le débat concernant l'identité africaine sur le terrain d'une essence spécifiquement Noire. Fanon s'opposera aux tenants de la Négritude, se disant contre cette essence Noire, et prônera plutôt une culture nationale. Par la suite, la question du métissage émergera telle que formulée initialement par Deleuze et Guattari, d'après la métaphore botanique du rhizome. Enfin le débat concernant l'appropriation de la langue et les échanges démontre que «l'africanité» n'est pas une essence mais bien une construction à la fois individuelle et collective sans réponse fixe, selon Bachir Diagne.

La difficulté à identifier clairement «l'africanité» nous amène sur un autre terrain qui est 
également très problématique, à savoir celui du théâtre africain. Et nous sommes amenés à nous poser la question: comment est-il possible de définir le théâtre africain? Nous pensons bien sûr aux rituels, aux cérémonies, aux contes et à la présence des griots, marabouts et autres figures qui servent à transmettre la parole. Mais ce théâtre s'est transformé et continue de se transformer comme nous le démontre le travail de la jeune génération de dramaturges africains, où les personnages ne sont plus ou ne sont pas nécessairement «Noirs» ou africains, où le griot n'existe plus et où l'Afrique n'est plus le lieu où se déroule l'action. Ce théâtre est en fait le fruit de nombreuses rencontres, de multiples chocs avec l'époque actuelle. Cette vision du monde et du théâtre est celle notamment préconisée par Koffi Kwahulé. Une analyse de son œuvre nous permettra de mieux cerner les tensions textuelles qui font écho à la violence et aux relations de pouvoir qui sont monnaie courante dans nos sociétés actuelles.

\subsection{Le «nouveau» théâtre «africain»}

Il faut évidement se méfier de certaines perceptions européocentristes qui estiment que le théâtre africain n'émerge véritablement que depuis la période coloniale et, surtout, postcoloniale, grâce aux contacts avec l'Europe et sa tradition théâtrale. Par exemple, Jacques Scherer, ${ }^{22}$ dans son livre Le théâtre en Afrique noire francophone, écrit que «le théâtre africain est jeune» (5) et qu'«en Afrique, il n'y a pas, ou pas encore, de tradition théâtrale» (9). Nous ne croyons pas à ces affirmations, car il s'agit plutôt d'une perception colonialiste selon laquelle la notion de théâtre, telle que réalisée par les Africains, doit correspondre à des classements ou à des catégories européennes. Le théâtre africain n'est pas jeune, bien au contraire. Scherer estime que la tradition orale n'a pas produit de théâtre et que celui-ci existe uniquement à partir du texte écrit. Selon lui: «En Afrique, le conteur appartient au passé et il a souvent cédé la place au véritable théâtre» (54). Étrangement, Koffi Kwahulé considère lui aussi que le théâtre ivoirien, d'expression

22 Jacques Scherer est spécıalıste du théâtre classıque et néoclassıque. 
française précise-t-il, est effectivement jeune:

D'une certaine manière, l'idée de bilan est associée à ce qui est relativement ancien, or le théâtre ivoirien d'expression française est jeune, tellement jeune que Dadier qui écrivit la première pièce ivoirienne Les Villes, est encore vivant. L'intention n'est donc pas de raconter une histoire qui n'a pas eu le temps de se faire, mais de mettre en question les premiers pas de ce théâtre dont on exige déjà une démarche assurée.

\section{(Critique du théâtre 7)}

Dans son livre Du rituel au théâtre-rituel, Marie-José Hourantier insiste, pour sa part, sur le fait que les tragédies grecques n'ont rien à voir ni avec le Kotéba ${ }^{23} \mathrm{ni}$ avec le rituel d'Abyssa, ${ }^{24}$ mais bien que ces formes théâtrales soient différentes, elles appartiennent toutes au théâtre. Elle confirme donc que la signification du théâtre et ses catégories changent et se transforment au fil du temps, des expérimentations et des pratiques.

On pourrait rétorquer aussi que le théâtre occidental d'aujourd'hui comme les expériences du Living, des Happenings... n'a rien à avoir avec la définition originelle du théâtre. Et Rousseau ne disait-il pas déjà qu'il ne saurait y avoir une définition universelle du théâtre parce que l'expérience culturelle de la France du XVII ${ }^{\mathrm{e}}$ siècle ne pouvait être l'expérience culturelle de tous les peuples? (D'après Lettre à $D^{\prime}$ Alembert, 1758). (36) ${ }^{25}$

De D’Alembert au théâtre des années 1970, les catégories théâtrales se sont modifiées considérablement. Depuis les diverses expériences dans le domaine du «théâtre-rituel» aux ÉtatsUnis et en Europe, de nouveaux critères de recherche théâtrale ont émergé. Nous pouvons

23 Le Kotéba est une forme de théâtre populaıre de la société bambara au Malı, où les instruments de musıque occupent une grande importance Il raconte les hauts et les bas de la vie du village et peut inclure des chants, des paroles, de la danse ou tout autre forme d'expression Selon Gaoussou Diawara dans Panorama critıque du théâtre malıen dans son évolution, Koté sıgnıfie «organısatıon» et «escargot» et ba sıgnifie «masse» et "grandeur» (20) 24 En Côte d'Ivorre, le rituel d'Abyssa est une danse sacrée annuelle de purıficatıon du peuple Nzıma Kotoko qui est liée à la tradition orale, selon Grekou Zadı

25 Selon la note (2) en bas de la page 36 du livre Du rituel au théâtre-rituel 
mentionner, entres autres, Julian Beck (The Living Theater), qui s'est intéressé aux cérémonies hassidiques juives. Ainsi que Jerzy Grotowski, dont l'une des orientations de son théâtre est inspirée du rituel de possession de la religion vaudou à laquelle il a été initié lors d'un séjour en Haïti, et dont le processus de formation des acteurs est lié au théâtre gestuel du Kathakali. Mentionnons aussi Peter Brook, qui a observé des rituels africains et qui a mis en scène l'épopée Mahâbhârata indienne, en s'inspirant des formes traditionnelles du drame dansé indien. Toutes ces expériences ont démontré un rapprochement certain avec le théâtre traditionnel africain. Depuis leurs recherches sur l'importance du corps comme site de création et sa prédominance par rapport au texte dans le théâtre occidental, les concepteurs scéniques tels que Brook, Grotowski et Beck, ont, entre autres, joué un rôle important quant à la transformation des catégories d'un certain théâtre occidental et quant au renouvellement des catégories de ces pratiques dites «scéniques».

Le théâtre de Koffi Kwahulé ainsi que celui de ces autres auteurs afro-européens, se retrouvent aux confluents de ces transformations. Une étude plus approfondie de la dramaturgie de Kwahulé nous permettra de cerner ce nouveau théâtre réalisé par des Africains installés en Europe. Nous pouvons affirmer que Koffi Kwahulé, originaire de la Côte d'Ivoire mais vivant en France, est un dramaturge africain dans la mesure où nous admettons et acceptons ces renouvellements et redéfinitions qui touchent tant à «l'africanité» qu’à la théâtralité.

Au cours des deux chapitres suivants, nous nous pencherons sur l'œuvre dramaturgique de Koffi Kwahulé. Nous verrons que les questions d'identités relatives à la peau noire sont présentes et qu'elles constituent l'un des fondements même du théâtre de Koffi Kwahulé. Mais nous verrons aussi la manière dont le dramaturge engage plutôt les mythes qu'il transforme au sein d'une société aux prises avec des êtres s'inscrivant dans le paradigme des exclus et des marginalisés de la société française. La notion même de «Noir» acquiert une nouvelle signification et comprend également les immigrants et tous ceux qui sont marginalisés. Les conflits n'émergent pas à cause 
de la couleur de la peau mais de par la présence même de ces êtres qui bousculent l'ordre apparent des choses, dans des sociétés qui peinent à affronter et à s'adapter aux changements. Si dans certaines pièces plus anciennes, la couleur de la peau des personnages est clairement définie par l'auteur, il n'en est pas toujours ainsi au sein du reste de son corpus. Cette couleur, cette condition Noire, n'est qu'un signe, parmi d'autres, qui symbolisent l'exclusion, la différence, la peur de l'Autre, cet étranger qui est prêt à tuer, à violer, à terroriser. Dans sa position de thèse, Virginie Soubrier résume ainsi cette idée liée à la couleur de la peau au sein de l'œuvre de Koffi Kwahulé. «L'auteur afro-européen n'oblitère pas la question raciale, mais il l'inclut dès lors dans un questionnement plus large, post-racial, sur l'altérité et la possibilité, à l'ère de la globalisation, d'un avenir commun» (Voix afro-européenne).

Dans ce premier chapitre qui traitait de l'africanité, nous avons tenté de définir ce qu'étalt un auteur afrıcain. La réponse à cette question est proposée par Koffi Kwahulé dans une forme très particulière, soit à travers son écriture théâtrale qui s'adresse à ces débats sur l'afrıcanité de manière très personnelle. La discussion précédente sur l'africanité nous semblait importante puisqu'elle ouvre le champ de réflexion des auteurs tels que Koffi Kwahulé, José Plya et Caya Makhélé, qui ne mettent pas nécessairement en scène des personnages «Noirs» mais bien des problématiques qui touchent des questions de pouvoir. Koffi Kwahulé devient l'une des figures emblématiques de cette réflexion identitaire des auteurs d'origine africaine qui vivent en France.

Pour examiner la question, nous allons essayer de cerner la vision kwahuléenne de la société dans laquelle il vit. Pour y parvenir, nous allons étudier cette transformation qui semble avoir lieu au sein de grands mythes fondateurs de notre société. Nous tenterons également de voir la manière dont Koffi Kwahulé s'est approprié les mythes fondateurs de la société judéochrétienne et comment ces mythes ont acquis de nouvelles significations dans le contexte de sa création théâtrale. Nous identifierons les différents éléments constitutifs du mythe en général et tenterons de cerner la manière dont le dramaturge représente ces mythes. Notre analyse portera 
sur six pièces qui sont, à notre avis, les plus emblématiques quant à leur appropriation et à leur transformation, qui semblent jouer un rôle fondamental dans leur conception. Il ne s'agit pas d'une analyse chronologique des pièces mais plutôt d'une étude portant, entre autres, sur les diverses formes de désacralisation des mythes lorsqu'ils sont replacés dans le monde contemporain. En effet, la structure des mythes reste souvent la même mais le contexte culturel et la relation entre les personnages changent. À titre d'exemple, dans la pièce Bintou, la figure du Christ en croix devient celle d'une jeune fille d'origine africaine vivant en France et qui meurt à la suite d'une cérémonie d'excision. Dans l'ensemble du corpus de Koffi Kwahulé, les personnages sont liés à la structure du mythe judéo-chrétien, tout en étant aux prises avec des faiblesses humaines. Il ne s'agit donc pas d'êtres transcendants, car ces personnages passent nécessairement par un processus de désacralisation qui aboutit à une nouvelle mythologie urbaine. 


\section{CHAPITRE 2: LE MYTHE SUBVERTI}

Dans le premier chapitre, nous avons tenté de définir le concept d'africanité qui, comme nous l'avons vu, demeure une notion très instable qui est difficile à cerner. Nous avons notamment relevé certaines des idées avancées par le mouvement de la négritude où le «Noir» constitue une essence. Nous avons également vu que cette approche a été vivement contestée par Fanon.

Dans ce second chapitre, nous aimerions analyser quelques pièces de Koffi Kwahulé afin de démontrer comment le dramaturge aborde les questions identitaires en lien avec la couleur de la peau. En fait, nous verrons que la question raciale, bien que présente dans certaines de ses premières pièces, fait rapidement place à un questionnement beaucoup plus vaste à propos des exclus de la société française, tels qu'observés par l'auteur. Ces exclus peuvent également prendre plusieurs formes. Nous pouvons penser aux «Nours», mais aussi à tous ceux qui sont étrangers, immigrants, pauvres, sans éducation, marginalisés, jeunes de la banlieue, jeunes filles qui deviennent chef de gang, sans oublier le fait qu'il existe des «Noirs» qui sont aussi Français.

Les pièces seront regroupées au sein des chapitres deux et trois. Le chapitre deux sera consacré aux mythes subvertis avec les pièces Cette vieille magie noire, Bintou et Ave Maria, alors que le chapitre trois traitera des cuvres qui incarnent les cérémonies du pouvoir et la mythologie de la victime. Dans ce chapitre, il sera question des pièces intitulées Big Shoot, BlueS-cat et Misterioso-119. Étant donné l'étendue du corpus dramaturgique de Koffi Kwahulé, nous avons choisi quelques pièces qui nous semblent plus emblématiques puisqu'elles représentent l'éventail des textes fondateurs des grandes mythologies de la civilisation judéo-chrétiennenotamment l'Ancien et le Nouveau Testament de la Bible—et qu'elles constituent par là, le point de départ de leur reconfiguration moderne. Nous voulons analyser la manière dont Koffi 
Kwahulé remet en question les mythes fondateurs judéo-chrétiens en transformant les paraboles, les allégories et les éléments qui constituent le fond des récits bibliques.

Koffi Kwahulé reprend, au sein de sa dramaturgie, ces récits fondateurs pour y inscrire des éléments qui alimentent les conflits des sociétés modernes tels que les confrontations sur les questions raciales, les conflits ethniques, les rapports sexuels, l'immigration et la violence dans les grands centres urbains. Ainsi, il resitue les personnages du texte biblique dans un tout nouveau contexte, transformant ainsi leur signification en leur attribuant de nouvelles dynamiques.

\subsection{Définitions du mythe}

Avant d'analyser la question des mythes dans les pièces de Koffi Kwahulé, il faudrait aborder brièvement les définitions du mythe telles que formulées par Claude Lévi-Strauss dans Anthropologie structurale, Roland Barthes dans Mythologies et René Girard dans Des choses cachées depuis la fondation du monde.

\subsubsection{Le mythe selon Lévi-Strauss}

Dans Anthropologie structurale, Lévi-Strauss accorde une véritable primauté à «l'histoire qui est racontée» (232), au récit et au contenu de ce récit. Selon lui, la valeur même du mythe n'est pas altérée par la traduction, pas plus que par la connaissance ou l'ignorance de la langue et de la culture où il se retrouve. Un mythe existe en tant que structure narrative et est perçu en tant que tel par tous. «La substance du mythe ne se retrouve ni dans le style, ni dans le mode de narration, ni dans la syntaxe, mais dans l'histoire qui est racontée» (232).

Lévi-Strauss ajoute que la permanence de la structure du mythe se rapporte toujours aux événements du passé. Toutefois, le récit mythique, c'est-à-dire ce qui est raconté, est atemporel, car il peut indiquer le passé, le présent ou le futur. Selon lui,

Un mythe se rapporte toujours à des événements passés: «avant la création du 
monde», ou «pendant les premiers âges», en tout cas, «il y a longtemps». Mais la valeur intrinsèque attribuée au mythe provient de ce que les éléments, censés se dérouler à un moment du temps, forment aussi une structure permanente. Celle-ci se rapporte simultanément au passé, au présent et au futur. (231)

Ainsi, les mythes possèdent une structure permanente et évoluent dans un espace-temps qui n'est pas défini. Ils font partie de la langue et relèvent du discours. En effet, ces récits fondateurs expliquent le monde parce qu'ils renvoient à tous les mythes dans toutes les cultures.

Le mythe existe uniquement en tant que phénomène transmis par la langue, qu'il s'agisse d'une langue orale ou écrite. Le champ de cette transmission est donc ouvert à de multiples formes et possibilités d'interprétation.

Lévi-Strauss traite de la reconstruction du mythe, qu'il définit comme étant les mythèmes qui sont les unités constitutives du mythe. Il ne s'agit pas de «relations isolées, mais des paquets de relations, et que c'est seulement sous formes de combinaisons de tels paquets et les unités constitutives acquièrent une fonction signifiante» (233-234). En effet, ce sont les agencements de ces paquets qui donnent de nouvelles significations aux mythes. Ces reconstitutions sont rendues possibles justement grâce aux mythèmes qui, par exemple, renvoient à des éléments précis des pièces de Koffi Kwahulé et du mythe judéo-chrétien. Toute cette notion de la reconstruction du mythe de Lévi-Strauss sera essentielle quand nous analyserons les pièces du dramaturge.

Dans la pièce Bintou, ces éléments constitutifs sont les mythèmes judéo-chrétiens qui se retrouvent dans la crucifixion de la jeune fille et dans la trahison de l'oncle et de P'tit Jean qui livre Bintou à l'exciseuse. En effet, la cérémonie d'excision à laquelle aboutit la pièce renvoie à la crucifixion du Christ sur la croix, l'ensemble de cette «aventure» étant une sorte de Calvaire auquel la jeune fille est soumise. Comme le Christ, Bintou sait que sa fin est proche lorsqu'elle affirme qu'elle n'atteindra pas l'âge adulte, mais que cela ne la dérange pas. Dans la pièce, la trahison de Judas, l'apôtre qui a livré Jésus pour trente pièces d'argent, devient multiple. Bintou 
est d'abord trahie par l'un des membres de sa famille, l'oncle Drissa, un vieux libidineux qui essaie de s'approcher d'elle, et puis par P'tit Jean, un voyou qui l'idolâtre bien qu'il n'ait pas réussi à joindre les rangs de sa bande parce qu'il consomme de la drogue. Pour retenir les services de P'tit Jean, l'oncle lui remet de la drogue et de l'argent afin que le jeune homme achète un revolver et s'assure de capturer Bintou.

Lévi-Strauss considère également qu'il est nécessaire «de définir chaque mythe par l'ensemble de toutes ses versions» (240). De plus, il faut tenir compte des différentes versions du mythe. Le mythe peut donc subir des relectures qui sont différentes. «Puisqu'un mythe se compose de l'ensemble de ses variantes» (240), il faudrait «les considérer toutes au même titre» (240). Nous entrevoyons ici ce qui donne toute la légitimité à la démarche de Koffi Kwahulé qui, nous le verrons, transforme radicalement les mythes en leur attribuant une nouvelle signification. Lévi-Strauss note que, malgré les multiples variantes, il est possible d'établir certaines grandes lignes du mythe.

Il insiste sur le fait de ne jamais omettre aucune de ses variantes, car elles sont toutes importantes et qu'elles se valent toutes. «Il n'existe pas de version «vraie» dont toutes les autres seraient des copies ou des échos déformés. Toutes les versions appartiennent au mythe» (242).

Ainsi, les mythes peuvent être regroupés, classés et mis en relation les uns avec les autres. Les structures qui les gouvernent tendent donc vers quelque chose de plus universel. Lévi-Strauss s'intéresse aux éléments qui convergent dans cette direction. Il ne tient pas compte de ce qui peut différencier les mythes tels que les lieux, les époques, les personnages, les langues. Il s'attarde plutôt à leur donner une interprétation d'ensemble. Comme nous l'avons vu, Lévi-Strauss cherche à déterminer les grandes lignes qui unissent toutes les variantes du mythe. Nous voyons déjà que la démarche de Koffi Kwahulé est parfaitement légitime. Il puise dans les formes mythiques pour écrire ses pièces. Les personnages qu'il met en scène lui permettent de repenser les relations mythiques dans le monde actuel, d'où cette écriture que nous pouvons associer à la subversion 
des mythes.

\subsubsection{Le mythe selon Barthes}

Chez Barthes, le mythe est davantage une parole orale et un système de communication. Il insiste plutôt sur la forme utilisée dans la transmission du mythe, c'est-à-dire l'oralité. Dans Mythologies, il explique que, de par sa forme, le mythe peut porter en lui plusieurs discours. «On voit qu'il serait tout à fait illusoire de prétendre à une discrimination substantielle entre les objets mythiques: puisque le mythe est une parole, tout peut être mythe, qui est justiciable d'un discours. Le mythe ne se définit pas par l'objet de son message, mais par la façon dont il le profère» (181).

Puisque «tout peut être mythe» (181), cette parole peut traiter des mythes de la société française contemporaine tels que la nouvelle DS de Citroën, le Tour de France comme épopée ou le visage de Garbo. Dans ce dernier exemple, il estime que «le visage de Garbo est Idée» (67) alors que l'actrice a toujours refusé de vieillir devant les caméras et les objectifs des photographes car «il ne fallait pas que l'essence se dégradât, il fallait que son visage n'eût jamais d'autres réalités que celle de sa perfection intellectuelle, plus encore que plastique. L'Essence s'est peu à peu obscurcie, voilée progressivement de lunettes, de capelines et d'exils; mais elle ne s'est jamais altérée» (66).

Dans cette logique, les événements ou les récits qui deviennent la matière du théâtre de Koffi Kwahulé sont aussi la matière très légitime du mythe contemporain, puisque tout peut être mythe. Il traite notamment de la tension et de la violence dans les banlieues françaises et de la langue qui y est parlée dans la pièce Bintou. Il en est de même avec le spectacle de téléréalité de Big Shoot puisque ces émissions sont très populaires à la télévision, car les personnalités qui sont mises en vedettes ne sont plus des comédiens professionnels mais plutôt des gens ordinaires qui bénéficient d'une célébrité instantanée. Ce faisant, ces événements ou phénomènes qui sont présentés par le dramaturge sont tout aussi légitimes que le visage de Garbo, alors que cette 
dernière ne voulait pas vieillir devant les caméras et dans l'imaginaire du public.

Selon Barthes, le mythe est un discours associé aux images de la société mais ce discours n'est pas forcément une forme d'écriture ni même une parole, une forme d'expression d'oralité ou une écriture. «Cette parole est un message. Elle peut donc être bien d'autre chose qu'orale; elle peut être formée d'écritures ou de représentations: le discours écrit, mais aussi la photographie, le cinéma, le reportage, le sport, les spectacles, la publicité, tout cela peut servir de support à la parole mythique» (182). La parole mythique n'est pas nécessairement une parole ni un discours isolés, mais elle peut être mise en valeur par un support autre tels que le cinéma, l'activité sportive, la photographie ou le reportage journalistique.

La parole mythique peut également se retrouver dans des contextes qui sont très spécifiques à notre société contemporaine, tel le spectacle théâtral qui véhicule les mythes de la société moderne. Koffi Kwahulé emploie ce passeport mythique de manière très efficace, d'autant plus qu'il exıge la présence d'un public pour le réaliser. Il ne s'agit pas d'une parole linguistique mais bien d'une «parole théâtrale». Dans ce texte, c'est la langue de la rue qui se fait entendre alors que les jeunes sont les principaux protagonistes. Le dramaturge y décrit la modernité urbaine en France où les jeunes obéissent à leur chef, au nom de la bande, et où les rivalités entre jeunes, le commerce de la drogue, la violence, la sexualité et les problèmes familiaux peuvent être la réalité de certains de ces adolescents.

Selon Barthes, les mythes peuvent naitre, se transformer ou mourir puisqu'ils vivent au gré des transformations de la société ou du temps qui passe. C'est pourquoi il existe actuellement les mythes de la femme Barbie, de la minceur chez la femme ou bien des extraterrestres qui n'existaient pas il y a un siècle. Selon Lévi-Strauss, les mythes sont universels alors que pour Barthes, ils sont instables car ils changent, tout comme la société: ils vont et viennent et ils ne peuvent donc pas tout dire à la fois. Associées aux mythes, ces transformations permettent donc des relectures constantes par les auteurs. Selon Barthes: 
Évidemment, tout n'est pas dit en même temps: certains objets deviennent proie de la parole mythique pendant un moment, puis ils disparaissent, d'autres prennent leur place, accèdent au mythe. ... on peut concevoir des mythes très anciens, il n'y en a pas d'éternels; car c'est l'histoire humaine qui fait passer le réel à l'état de parole, c'est elle et elle seule qui règle la vie et la mort du langage mythique. (182)

Cette instabilité dans les mythes provient du fait qu'ils changent, tout comme la société change et évolue. Dans la pièce Bintou, il est question de la sexualité d'une jeune fille de 13 ans qui n'est plus une enfant mais qui n'est pas encore une adulte, et dont les vêtements et les actions ne laissent aucun doute quant à son très grand pouvoir de séduction et à son autorité sur sa bande de jeunes hommes tous plus âgés qu'elle. En fait, ce sont des exemples comme celui-ci qui illustrent de nouvelles transformations de la société, qui amènent de nouveaux mythes et qui font en sorte qu'1l n'y a «aucune fixité dans les concepts mythiques: ils peuvent se faire, s'altérer, se défaire, disparaître complètement. Et c'est précisément parce qu'ils sont historiques, que l'histoire peut très facilement les supprimer» (193).

\subsubsection{Le mythe selon Girard}

Après Lévi-Strauss et Barthes, il est également important d'évoquer les travaux de René Girard, qui contiennent de multiples éléments que l'on peut associer aux pièces de Koffi Kwahulé. Dans Des choses cachées depuis la fondation du monde, René Girard définit le mythe comme étant «un texte faussé par la croyance des bourreaux en la culpabilité de leur victime» (223). Il ajoute également que les mythes «incarnent le point de vue de la communauté réconciliée par le meurtre collectif» (223). Le résultat attendu est donc la réconciliation de cette communauté qui se rassemble autour du meurtre collectif et dont toute la société est responsable. Le mythe serait donc l'explication d'un événement qui justifie l'existence même de cette communauté et, à l'origine de ce récit fondateur, il y aurait un meurtre rituel. 
Ce meurtre est nécessaire, voire essentiel pour la divinité. La société réconciliée est «unanimement convaincue qu'il s'agit là d'une action légitime et sacrée, voulue par la divinité elle-même, et qu'il n'est pas question de répudier, de critiquer» (223-224). En effet, selon Girard, rien n'est remis en question. Il n'est pas naturel de critiquer ceux qui ont commis ces meurtres, ces gestes étant considérés comme nécessaires.

Cette acceptation du meurtre a des conséquences importantes. «Cette attitude ne peut manquer d'avoir des répercussions non seulement sur la mythologie mais de proche en proche sur tout ce qu'entraîne le fondement caché du meurtre collectif, les rituels, les interdits et la transcendance religieuse» (224). Étant donné que le mythe n'est pas remis en question, «ce sont toutes les formes et les valeurs culturelles, mêmes les plus éloignées des mythes en apparence, qui devraient être affectées» (224).

Le modèle de Girard est évidemment la mise à mort du Christ. Gırard explique que la communauté est rassemblée par le meurtre collectif qui est, en fait, le meurtre du Christ, personnage transcendant, qui réussit à unifier cette communauté autour du meurtre originel. Il faudrait dire, cependant, que le Christ était un jeune homme persécuté par ses coreligionnaires et qu'il n'est devenu un être transcendant qu'après sa mort. Dans Bintou, Koffi Kwahulé renverse le mythe. La victime n'est plus le fils de Dieu mais plutôt une jeune fille qui dirige une bande de garçons et qui est persécutée par la société. Elle était un être transcendant avant sa mort.

Dans la pièce Big Shoot, il y a une violence, toutefois elle n'est pas perçue comme nécessaire mais plutôt abusive. Le public scénique, celui qui fait partie du texte dramaturgique et se retrouve en scène en train de regarder le spectacle dans le spectacle, ne remet pas en question le meurtre, contrairement au public qui assiste à la pièce dans la salle de théâtre et qui lui, est choqué par cette violence. Ce public près de nous le remet immédiatement en question. Il en est de même avec la pièce Blue-S-cat où a lieu une mise à mort rituelle. La conclusion, où la femme tue l'homme, est difficile à comprendre car elle ne va pas de soi. L'interprétation de Koffi 
Kwahulé choque le public dans la salle. En fait, le public dans la pièce fait partie du public réconcilié par le meurtre, il fait partie du mythe. Mais le dramaturge oblige le spectateur dans la salle à remettre en question ce qu'il voit, à être perturbé et à ne pas comprendre ce qui se passe sur scène, car il évolue en dehors de ce monde présenté. Les choses ne vont pas de soi et nous verrons que cette approche de Koffi Kwahulé semble être au cœur de sa démarche dramaturgique, dans laquelle l'aliénation du public qui se trouve dans la salle contribue à la subversion des mythes.

\subsubsection{Résumé des arguments autour du mythe}

Nous avons vu que les pièces du dramaturge répondent à la fois aux définitions du mythe telles que proposées par Lévi-Strauss, Barthes et Girard. Rappelons que le premier s'est surtout intéressé à l'histoire qui est racontée, car il s'agit d'un récit composé des éléments qui constituent la structure de base du récit mythique. En effet, Lévi-Strauss insiste sur la nature universelle du mythe, sur le fait qu'il se rapporte toujours à un événement du passé, que ce qui est raconté est atemporel, qu'il fait partie de la langue et relève du discours, et qu'il n'existe pas de versions «vraies» du mythe. Tous ces éléments légitimisent la démarche de Koffi Kwahulé puisqu'un mythe n'appartient ni à un seul moment, ni à une seule culture et encore moins à une seule société, car il se retrouve dans toutes les cultures. Nous pouvons retrouver des structures, qui sont en fait des unités constitutives appelées mythèmes qui structurent et resituent les mythes dans de nouveaux contextes.

Selon Barthes, le mythe est associé à la parole et à l'instabilité du mythe qui se transforme, au même titre que la société contemporaine. Il s'agit d'un phénomène qui émerge dans l'actualité. La parole mythique est un message qui peut bénéficier de différents supports selon les formes actuelles de communication et qui peut donc être livré par de nombreux moyens tels que la photographie, le cinéma ou le reportage. Il ne s'agit pas d'une parole ou d'un discours isolé. Tout 
peut ainsi devenir matière du mythe. Cela aussi légitimise la démarche de Koffi Kwahulé.

Girard, pour sa part, trace un lien direct entre le mythe et la communauté réconciliée par le meurtre collectif. Ce geste, accepté de tous, n'est donc pas remis en question puisqu'il est jugé essentiel par la divinité. Il met en présence un bourreau et une victime, ainsi que toute la communauté qui se rassemble autour de ce meurtre.

Les pièces de Koffi Kwahulé s'inscrivent dans la définition de Lévi-Strauss, en ce sens que le dramaturge s'inspire des mythes fondateurs judéo-chrétiens afin d'en proposer de nouvelles versions. Dans la pièce Blue-S-cat, l'Ange exterminateur prend les traits d'une femme qui tue un inconnu, alors qu'ils sont tous deux coincés dans un ascenseur en panne. Dans Misterioso-119, il s'agit d'une version renouvelée de la Cène et de l'Eucharistie, alors que des prisonnières tuent et mangent une intervenante en théâtre au sein de la prison. Dans Ave Marla, il est question de la messe du rite catholique et de la présence d'un frère venu de l'étranger, quı en fait, n'est autre que Caïn, celui qui volera la femme d'Abel. Bintou est une pièce où une jeune fille africaine devient une victime expiatoire de la banlieue, car elle refuse de se soumettre à l'autorité des parents et à l'excision qui lui est imposée.

La réécriture de ces mythes d'inspiration biblique leur confère alors de nouvelles significations, d'où cette subversion des mythes que nous analyserons tout au long de ce chapitre.

La définition du mythe selon Barthes se retrouve également dans le théâtre de Koffi Kwahulé. Rappelons que Barthes affirme que «puisque le mythe est une parole, tout peut être mythe, qui est justiciable d'un discours» (181). Les pièces de Koffi Kwahulé s'inscrivent au confluent de plusieurs discours, qu'ils soient à la fois littéraire et théâtral. Ils sont inévitablement marqués par une vision postcoloniale qui situe ces récits dans de nouveaux rapports de pouvoir, où ceux qui subissent de mauvais traitements ne sont pas nécessairement ceux qui ressemblent aux victimes traditionnelles de nos sociétés occidentales. En ce qui concerne les rapports de pouvoir où les contextes sont très variés (Cette vieille magie noire, Bintou, Ave Maria, Big Shoot, 
Blue-S-cat et Misterioso-119), la mort des victimes symbolise leur exclusion d'un monde où ils n'ont pas de place. Leur présence est en soi une forme de transgression et ils sont condamnés à disparaître ou à subir les pires conséquences de leur intrusion dans un monde qui leur est hostile.

Nous verrons également la manière dont Koffi Kwahulé transforme les mythes en créant de nouveaux archétypes, comme le sont Faust, Phèdre ou CEdipe, que Patrice Pavis associe à des personnages-archétypes. L'archétype constitue alors «un type de personnage particulièrement général et récursif, dans une œuvre, une époque ou dans toutes les littératures ou les mythologies» (42).

À la lumière de ces différentes interprétations du mythe, nous allons étudier la manière dont Koffi Kwahulé s'approprie les mythes afin de les transformer en leur attribuant de nouvelles significations. Nous avons rassemblé ces trois pièces dans ce deuxième chapitre parce qu'elles s'inscrivent toutes dans un cadre spécifique, soit des réalités actuelles contemporaines: Cette vieille magie noire se déroule à New York, dans le monde de la boxe et du jazz. Dans Bintou, il s'agit de la vie des immigrants dans les banlieues d'une ville francophone, sans doute Paris. Enfin, l'action d'Ave Maria se déroule dans la ville de Québec, ce «Nouveau-Monde» bien ancré dans la religion catholique.

\subsection{Cette vieille magie noire et le mythe de Faust}

Le mythe de Faust se caractérise par sa relative jeunesse si nous le comparons aux mythes de l'Antiquité ou à ceux associés à la Bible. Il s'agit d'un mythe populaire qui date du $\mathrm{XV}^{\mathrm{e}}$ et du $\mathrm{XVI}^{\mathrm{e}}$ siècle. Il est souvent associé à un étudiant allemand qui serait devenu médecin, astrologue, philosophe et magicien. Le mythe du Docteur Faust s'est surtout fait connaître à travers les cuvres de Marlowe mais surtout par les textes de Goethe. Trois grandes séquences se retrouvent dans l'Urfaust de Goethe qui est, dans les faits, la première version de l'œuvre. Voici Faust où se jouent le drame du savant qui signe un pacte avec le démon, la satire de l'université et la tragédie 
de Marguerite / Gretchen. Le vieux Faust vend son âme à Méphisto et obtient, en retour, la jeunesse, le pouvoir et l'amour de Marguerite, aussi connue sous le nom de Gretchen. En effet, grâce au Diable, Faust séduit la jeune femme et passera la nuit avec elle. La mère de Gretchen en meurt après avoir avalé la potion qui a permis aux amoureux d'être ensemble. De cette union naît un enfant que Gretchen, toujours très religieuse, tuera pour éviter le scandale. Son geste l'enverra en prison mais elle sera finalement admise au Paradis grâce à sa foi. Malgré sa grande érudition, Faust est voué à la damnation mais il sera lui aussi récupéré, puisque dans le Second Faust de Goethe, il sera à son tour «sauvé».

Le mythe est souvent associé au péché de la connaissance et vise l'accomplissement du corps et de l'esprit, alors que l'homme court à sa perte s'il refuse de s'inscrire au sein de la société à laquelle il appartient. En cela, il faut évidemment noter le rapprochement avec le récit d'Adam et Ève dans la Bible où ils goûtent le fruıt de l'Arbre de la connaissance sous l'influence du serpent. Ce geste leur fait connaître le Bien et le Mal et, du coup, leur ferme la porte du Paradis terrestre.

Dans Cette vieille magie noire écrite en 1991 (publiée en 1993), il est question de l'Amérique, de la boxe, du jazz et du mythe de Faust. Ici Koffi Kwahulé exploite une orientation différente du mythe, car le rapport entre Faust et le Diable est très particulier. La pièce met en scène un boxeur Noir américain, Shorty, qui a signé un pacte avec son manager Shadow, Méphisto, afin de s'assurer victoire après victoire et ainsi, être reconnu comme le champion Noir par l'Amérique Blanche. En tant qu'incarnation de Faust dans la peau de Shorty, ce personnage évolue dans un monde Blanc, où il court à sa perte en voulant faire du théâtre plutôt que de boxer pour défendre la cause afro-américaine.

Dans Cette vieille magie noire, la fable s'organise autour de douze tableaux: 1. Le pacte, 2. Le sang me rend fou. Pas le mien, celui des autres (Hagler), 3. Toute défaite est un combat de trop, 4. Un combat sans K.O., c'est comme un amour sans orgasme, 5. La cabale, 6. Le complot, 
7. Le "Grand Espoir Blanc", 8. Le livre, 9. Un crime parfait, 10. Est-ce qu'on peut être nègre sans être patient?, 11. La décision et 12. Ne juge pas le bonheur, ça porte malheur. Les

personnages sont divisés en deux groupes antagonistes, le premier étant celui de Shorty, le boxeur Noir et son équipe surtout formée de Noirs, et le second est associé à Todd Ketchel, un boxeur Blanc épaulé par son équipe majoritairement Blanche.

Shorty est le meilleur des boxeurs américains mais, pour y arriver, il a dû signer avec son sang une entente lui assurant argent, gloire et respect, avec son manager Shadow, l'incarnation du diable. Toutefois, Shorty rêve d'être comédien. Dans ses temps libres, sous la direction de son manager, il répète la pièce Faust où il joue aux côtés de sa sœur Angie qui est, elle-même, une chanteuse de jazz, l'incarnation de Gretchen. Mais un jour, lors d'un combat de boxe, Shorty tue Todd Ketchell, le «Grand Espoir Blanc». Cet homme arrogant a toujours joué la carte du racisme avant la tenue de ses combats, alors que Shorty, lui, s'y était toujours refusé. Shorty ne voulait pas que le combat se solde par la mort de son adversaire, mais Shadow en avait décidé autrement.

Par ailleurs, les rumeurs d'inceste entre Shorty et sa sœur deviennent de plus en plus importantes avant la tenue du combat contre Ketchell. Après le K.O. où Ketchell ne se relève plus, Shorty veut arrêter la boxe mais Shadow refuse de le libérer, menaçant même de s'en prendre à Angie s'il ne continue pas. Angie et Monsieur Jean, l'entraîneur de Shorty, s'arrangent pour mettre au point une histoire de dopage afin de mettre fin à la carrière du boxeur. Le scandale éclate et Shorty est hospitalisé sous prétexte qu'il a besoin de repos. Shadow rend visite au boxeur et lui apprend que sa sœur a perdu la tête en plein tribunal. Après son témoignage, elle a été internée dans un hôpital où elle affirmait que Shadow était le Diable, qu'elle était enceinte et que Shorty était le père de l'enfant à naître. Shorty affirme ensuite à son manager qu'il veut reprendre la boxe. Toutefois, avant que les choses ne reviennent à la normale, il faudra que Shorty retrouve son âme qui s'est enfuie lorsque le scandale a éclaté. Après avoir esquissé quelques mouvements de boxe, Shorty reprend de l'assurance. Les didascalies indiquent que des hommes en blouse 
blanche applaudissent, alors que Shorty et Shadow saluent. Puis tous «descendent, prennent les autres acteurs par la main et saluent tous le vrai public» (84).

Dans cette pièce, la couleur de la peau et le racisme sont des thématiques inséparables des notions de Bien et de Mal. Le titre même de la pièce marque implicitement la différence entre le Bien et le Mal, entre le Blanc et le Noir, et entre la magie blanche et la magie noire, cette dernière étant associée à la sorcellerie qui emploie le Mal pour faire le Mal. Shorty, le personnage principal, est un boxeur Noir d'un certain âge qui a scellé un pacte avec le Diable afin de toujours remporter la victoire dans le ring. Cette relecture du mythe européen de Faust est incarnée par la présence de Shadow, le manager Noir de Shorty, qui est littéralement une ombre si nous traduisons son nom en français. Shadow, qui est décrit comme un dandy intellectuel, est en fait Méphisto ou l'un de ses exécutants au sein d'une grande confrérie dont nous savons très peu de choses, comme sı le dramaturge tenait à laisser planer une sorte de mystère autour des agissements de cette communauté réunie autour du Diable. Lors d'un combat crucial, Shorty tue, sans le vouloir, son adversaire Todd Ketchel ${ }^{26}$, un ancien «Grand Espoir Blanc» et ami d'enfance.

Koffi Kwahulé semble vouloir dire que le salut des Noirs en Amérique peut se réaliser à travers une nouvelle moralité où le Diable est le plus puissant. Pour vaincre le pouvoir Blanc de «l'establishment», les Noirs ont besoin de la force du Diable, seule force qui puisse les aider, quitte à devoir brûler en enfer. Nous pouvons également y voir un renversement des valeurs du Bien et du Mal dans le monde chrétien. Avec ses allures de dandy ${ }^{27}$ intellectuel, Méphisto acquiert une nouvelle valeur positive. Il s'agit d'une autre forme de subversion, mise en place par Koffi Kwahulé, qui transforme le Diable en homme élégant.

Cette pièce dresse également un parallèle avec un véritable combat qui s'est tenu en 1965

26. Le personnage de Todd Ketchel peut faire penser à l'ancien champion de boxe Stanley Ketchel quı n'est pas mort sur le rıng mais assassıné dans un ranch en 1910. Ketchel, fils d'ımmıgrants polonais, avait hérité du surnom de Michigan Assassin

27 Il s'agıt d'une référence directe au jazz de la fin des années 1930 et du début des années 1940, à l'époque de Cab Calloway et de la mode des Zoot Sulters aux États-Unis et des zazous en France. 
et qui a opposé Muhammad Ali à Floyd Patterson. ${ }^{28}$ Il est donc question de l'identité américaine, de ce qui est américain et de ce qui ne l'est pas. Ces valeurs ont notamment trait à la couleur de la peau mais également aux croyances religieuses et aux bonnes mœurs qui sont nécessaires pour oser porter les couleurs du drapeau américain.

Dans cette pièce, Shorty devient une figure archétypale du Noir. Il n'existe que par sa négativité, dans son opposition à la société Blanche américaine et au boxeur Todd Ketchel. Cette idée renvoie directement à Fanon alors que Shorty ne peut exister en dehors de cette relation avec le «Blanc». Shorty est dépossédé de son identité à la suite de son pacte avec Shadow. Il gagnera inlassablement tous ses combats pour le bien de la grande «Communauté», comme le lui rappelle son entraîneur. Devenu étranger à lui-même, il est contraint de prendre le rôle d'idole et de martyr tout à la fois, souhaitant mettre fin à l'entente qu'il a pourtant scellée avec son propre sang. Il se retrouve au croisement de deux communautés quasi-imperméables l'une pour l'autre. Pour Shorty, jouer le rôle d'un personnage théâtral est sa manière d'incarner ce martyr Noir archétypal, de transcender la réalité et de devenir une nouvelle figure emblématique par le théâtre. La mort du boxeur Blanc, orchestrée par Shadow contre la volonté de Shorty, est une sorte de punition envers celui qui souhaite mettre fin à sa carrière et au pacte. Mais ce coup fatal qu'il porte à son adversaire entraîne le repli de Shorty qui refusait de tuer Todd Ketchel. Ce geste involontaire attise la haine des Blanc envers celui qui a tué l'ancien «Grand Espoir Blanc». Selon Sylvie Chalaye:

Shorty est l'emblème du héros noir divinisé et invincible qui donne du rêve «aux petits enfants», mais qui ne suffit pas à sortir du mépris le peuple noir tout entier. Sur le ring, il tue le Blanc, mais la mort du Blanc n'anéantit pas l'antagonisme que

28 Le 22 novembre 1965 à Las Vegas se déroule un important combat de boxe quı opposa deux boxeurs Noirs: Muhammad Alı (Cassıus Clay) et Floyd Patterson Ce dernier était alors vu comme le choix de l'establishment blanc amérıcaın afin de reconquérır le tıtre de champıon. Il affirma qu'ıl allatt regagner le tıtre pour l'Amérıque Cette remarque sıgnıfiatt donc qu'Alı n'étaıt pas un vraı Amérıcain puısqu'ıl s'était convertı à l'Islam. Alı remporta la victorre en 12 rounds, refusant de mettre fin au combat par un K.O Plusieurs reprochèrent à Alı sa cruauté et son manque de respect envers un boxeur très apprécié 
l'Histoire a instauré entre les deux races, et le boxeur blanc laisse une descendance, sa jeune épouse est enceinte, tandis que Shorty est inexorablement condamné à l'amour incestueux et stérile pour sa sœur. L'identité perdue du peuple noir se situe dans ce retour à l'humanité que tente Shorty. (Partir de combien 76-77)

Virginie Soubrier estime également que Shadow constitue «la figure archétypale de l'immigré noir» (Fraternité 192), un personnage sans véritable identité ou dont l'identité n'intéresse personne. Il est celui par qui la vengeance peut se concrétiser parce qu'il manipule Shorty à sa guise. Elle avance également que Shadow, ce personnage de l'ombre, immigré noir sans passé, est ««un impersonnage», avec l'espoir qu'un jour tombe le masque forgé par les Blancs» (195) et qu'il incarne ainsi «tous les aspects de la négativité du Noir» (197).

Bien que l'action de la pièce se déroule à New York, dans le pays du rêve américain, l'espace réservé aux Noirs semble n'émerger qu'à travers la boxe et la musique jazz afroaméricaine, laquelle a déjà été qualifiée de musique du diable. ${ }^{29}$ D'ailleurs, le jazz est très présent dans le texte puisque, dans sa présentation des personnages, l'auteur indique qu'un quartet de jazz est indispensable pour monter la pièce et que Angie, la sœur de Shorty, doit chanter des airs de jazz dans son cabaret.

Tous les combats de boxe de la pièce dépassent largement le cadre sportif puisqu'ils symbolisent la dualité qui existe dans cette société américaine à la fois Blanche et Noire. En effet, toute la question du racisme est au cœur du discours de Koffi Kwahulé, qui n'est pas sans rappeler certains éléments de la mythologie afro-américaine. Aux États-Unis, le Noir est un Africain transplanté par l'esclavage. Cet ancien esclave a ensuite passé toute sa vie en Amérique. «Je voulais écrire une pièce sur la mythologie noire contemporaine. C'est pourquoi j'ai eu recours à des choses facilement identifiables à la communauté noire. Au départ, pour moi, le jazz c'était

29 Le blues et le jazz ont été consıdérés comme des musıques du dıable. En Allemagne, le régıme nazı avait ınterdıt le jazz dans les années 1930, compte tenu de ses racines africaınes et du fait que les musiciens de jazz avaient des origines juives. 
la présence physique des musiciens» (Kwahulé et Mouëllic 19). Et ce personnage de Shadow, ombre inquiétante, n'est nul autre que le double négatif de Shorty qui, tel un chef d'orchestre, décide de la suite des choses.

Selon ses adversaires Blancs, Shorty demeure un Noir dangereux qu'il faut neutraliser pour le bien de la nation américaine. Le manager de Ketchel, McKenzie, résume bien toutes les inquiétudes des Blancs par rapport à leur adversaire, qui est bien plus qu'un simple boxeur mais plutôt une image du Noir qu'il faut anéantir. L'équipe de Ketchel lui demande de répéter la célèbre phrase de Floyd Patterson concernant son intention de ramener le titre en Amérique:

Pourquoi crois-tu que la boxe est d'abord un sport américain? Parce que le Blanc est le bon et le Noir le méchant, bien sûr. Mais cette fois, les choses ne sont pas aussi simples; Shorty n'est pas comme les autres boxeurs noirs. D'accord, il a pris à une certane époque des positions sur la question raciale, mais jamais elles n'ont atteint les outrances maladives d'un Cassius Clay. Il ne fricote pas non plus avec des femmes blanches comme ce vieux libidineux de Jack Johnson. Non, Shorty est le champion à la fois modeste, poli et mystérieux que toute l'Amérique blanche et noire, admire. Voilà le danger! C'est le parfait héros américain. Il faut donc brouiller les cartes et imposer un camp, le tien, à l'Amérique blanche. (59)

Ainsi, la provocation vient toujours du côté des Blancs clame McKenzie, qui reprend à son compte la phrase de Patterson. La question raciale est, selon le manager de Ketchel, celle qui prime. C'est pour cette raison qu'il n'hésite pas à évoquer et à provoquer la conscience de l'Amérique Blanche et de ses pulsions les plus morbides, afin qu'elle ouvre les yeux et qu'elle réalise que, derrière ce héros parfait, un Noir se délecte de sa toute-puissance sans que rien n'y paraisse. Le camp de Ketchel s'est donné comme mission de dénigrer et de détruire Shorty qui a, en quelque sorte, endormi l'Amérique Blanche grâce à son masque de champion parfait. Mais les deux clans ne luttent pas à armes égales, rappelle Koffi Kwahulé. S'il y a d'un côté, le talent 
sportif et la condition physique des protagonistes, il existe, de l'autre, des forces en présence qui dépassent le corps du boxeur pour atteindre la sphère des puissances occultes selon Cette vieille magie noire. Peut être signifie-t-il que le corps constitue l'instrument de prédilection du Noir qui ne peut que tricher pour arriver à ses fins, endormant par le fait même la conscience de l'Amérique Blanche.

Le titre de la pièce fait référence à la culture populaire américaine et à la population afroaméricaine, puisqu'il s'agit de la traduction littérale de la chanson «That Old Black Magic». ${ }^{30}$ Dans la chanson, la vieille magie noire dont il est question est en fait l'amour d'une personne pour une autre personne. L'interprète explique être sous le charme de son partenaire, comme s'il s'agissait d'un sort, et qu'il ne pouvait résister à la tentation. Il est possible de tracer de nombreux parallèles entre ce que la chanson évoque et la pièce de Koffi Kwahulé, dans laquelle Shorty est sous l'emprise de Shadow et où le boxeur entretient une relation incestueuse avec sa sœur.

Au cœur de cette relation qui transgresse tous les interdits, Angie, la sœur de Shorty, est elle aussi un personnage double. Celle qui est connue comme chanteuse de jazz est également comédıenne. Lors des répétitions de Faust, elle délaisse momentanément la musique pour le théâtre. Elle y tient le rôle de Gretchen face à son frère Shorty qui, lui, joue Faust, le rôle principal.

Dans la pièce de Goethe, Gretchen tombe enceinte de Faust, sans qu'ils ne soient unis par les liens du mariage. Gretchen étant une femme très religieuse, elle décide de tuer son enfant pour éviter le scandale. Elle est ensuite emprisonnée pour son geste. Faust essaie de la libérer mais celle-ci refuse, car personne ne peut plus rien pour elle.

Lors des répétitions de Faust dans la pièce de Koffi Kwahulé, Shorty et Angie doivent

30 «That Old Black Magıc» (1942) est une chanson de Harold Arlen (musıque) et de Johnny Mercer (paroles). Elle a été popularısée par Glenn Miller et a été reprise par tous les grands noms de la chanson et de la culture amérıcaine dont Ella Fitzgerald, Frank Sinatra, Jerry Lewıs, Marılyn Monroe, Sammy Davis Junıor, Tonny Bennett, Miles Davis et Peggy Lee 
s'embrasser car, à de nombreuses reprises, le frère et la sœur doivent jouer l'amour de Faust et de Gretchen, sous les ordres du metteur en scène Shadow qui les incite à s'embrasser davantage. La ligne devient ainsi très mince entre les gestes posés par les personnages de Faust- des gestes interdits par la morale de l'époque —et ceux qui sont dictés aux comédiens, un frère et une sœur dans la pièce de Kwahulé, des personnages sortis tout droit de l'imaginaire du dramaturge.

Cet inceste entre frère et sœur est interdit, tout comme la relation entre Faust et Gretchen. Mais ce tabou, qui est habituellement caché, privé, afin de ne pas être exposé à l'opprobre générale de la société qui condamne immanquablement cette relation, peut se dérouler ouvertement au théâtre, puisqu'il ne s'agit que d'un jeu de faux-semblant. Toutefois, cette relation sème le doute dans l'entourage du boxeur. C'est d'ailleurs ce qu'affirme Mickey, le sparringpartner de Shorty, qui travaille également pour le compte de l'équipe de Todd Ketchel. Il dit tout haut ce que tout le monde pense tout bas. La gravité des gestes reprochés renvoie directement à ceux qui ont mené Gretchen au bord du gouffre. «Ce qu'il y a entre eux ne me regarde pas, ce sont leurs oignons. Des choses trop graves en tout cas pour qu'on en juge. Dieu seul aura la force de les juger» (57).

Malgré les accusations publiques de Todd Ketchel qui sont portées à l'endroit du frère et de la sœur dans la presse, jamais ils ne se défendent. Ketchel parle même de pratiques qui vont à l'encontre de la civilisation chrétienne américaine, de valeurs qui sont opposées à celles des pionniers en ce qui concerne le travail, le courage, la démocratie, la liberté et l'amour. Le combat du «Grand Espoir Blanc» est celui de l'Amérique et donc de Dieu. Ici Ketchel, sans se prendre pour Dieu, incarne tout de même la droiture, la raison et les valeurs de l'Amérique Blanche. Ce Dieu / Ketchel / Blanc / Bien, s'oppose donc au pécheur impardonnable qu'est Shorty / Noir / Faust / Mal, dans cette lutte à finir entre les deux forces suprêmes qui séparent inexorablement les Blancs des Noirs.

Le racisme, toujours très présent tout au long de la pièce, fonctionne à double sens, tant 
du côté des Blancs que de celui des Noirs. Le personnage de Negus, un ancien boxeur Noir faisant partie du clan de Shorty, revêt une grande importance puisque c'est lui qui alimente, en partie, les tensions avec les Blancs. «Il avait la particularité de s'exprimer par paraboles et proverbes africains. ... On ignore aujourd'hui encore le rôle exact qu'il a joué auprès de Shorty, mais son influence sur la communauté noire de cette ville était incontestable» (11), explique le reporteur aux téléspectateurs au tout début de Cette vieille magie noire.

En effet, Negus semble incarner l'âme de cette communauté qui tente de se débarrasser du fardeau qui lui est imposé depuis toujours par cette société Blanche. Shadow résume ainsi les efforts des Noirs en vue d'obtenir la reconnaissance des Blancs. «Nous avons toujours été obligés de tout prouver, y compris que nous sommes des hommes, nous les fils aînés du monde!» (17). Angie rappelle elle aussi que les efforts nécessaires pour maintenir les Noirs à un niveau comparable à celui des Blancs doivent être répétés inlassablement. «Nous sommes NoirsAméricains... Ici tout le monde peut se permettre de perdre, mais pas nous: il n'y a de la place que pour les vainqueurs. N'oublie jamais que nous sortons de cet endroit où même l'espoir est une souffrance. N'oublie jamais cela, P'tit-Jazz. Nous sommes Norrs-Américains») (26). Cette réplique résume à elle seule le combat des Noirs qui ne peuvent se définir comme étant Américains uniquement. Ils doivent y accoler la couleur de la peau pour leur permettre d'exister aux côtés des Blancs qui, eux, sont Américains.

De son côté, Todd Ketchel accuse Shadow de racisme car ce dernier refuse, d'entrée de jeu, tout combat avec cet adversaire qu'il ne juge pas à la taille du sportif de race noire. «N'est-il pas de bon ton de justifier son propre racisme en invoquant je ne sais quel racisme noir» (15), affirme Shadow qui refuse toujours le combat. Le duel aura lieu après qu'il ait organisé deux combats truqués avec deux autres adversaires. Par la suite, pour déstabiliser son adversaire, il parlera du dégoût que lui inspire la liaison entre Angie et Shorty.

Les accusations d'inceste entre le frère et la sœur font en sorte qu'Angie, qui devait 
chanter l'hymne américain avant le combat, se voit refuser ce privilège parce qu'elle souillerait ainsi «The Star-Spangled Banner». Shorty sait que son manager a tous les pouvoirs et c'est pourquoi il dit à sa sœur: «Eh bien, tu souilleras l'hymne américain. Je te dis que Shadow s'en chargera. C'est toi qui le chanteras» (66), démontrant que le Diable a un pouvoir bien plus grand que celui de son adversaire. Mais lors de l'événement, son interprétation ne sera suivie d'aucun applaudissement, dans une sorte de lutte entre le Bien et le Mal. Nous voyons que l'inceste sert ici de porte d'entrée au racisme, qui est ainsi justifié, servant de rempart à la haine de certains Blancs qui tentent à tout prix d'annihiler le champion Noir.

Manfred Schmidt-Brabant estime que ce n'est pas sans raison que «dans cette œuvre poétique l'histoire de l'humanité est considérée selon les grands pans de son évolution culturelle» (9). En effet, Koffi Kwahulé reprend, dans un contexte contemporain, l'histoire de cette évolution au tournant du XX $\mathrm{X}^{\mathrm{e}}$ siècle où Blancs et Noirs s'affrontent dans une société profondément raciste, marquée par une domination historique et une libération qui s'est partiellement cristallisée, mais où il reste encore beaucoup à faire pour atteindre une véritable égalité sociale, politique et économique. Le combat entre Shorty et Ketchel est l'incarnation même de cette lutte. Toutefois, grâce à la présence de Shadow, le processus est dorénavant inversé, laissant présager une victoire tant attendue et un renversement des rôles, puisque c'est lui dorénavant qui manipule les personnages. Si le Faust de Goethe représente une initiation sous diverses formes, elle est, chez Kwahulé, l'initiation de tout un peuple qui se libère de l'emprise du Blanc et qui devient maître du jeu. Mais pour y arriver, il aura fallu s'associer au Mal et vaincre grâce à lui, tout en trichant pour y parvenir.

Toujours selon Schmidt-Brabant, l'un des principes primordiaux de l'initiation réside notamment dans la coopération des principes féminins et masculins où «l'être humain ne peut réaliser cette complétude sans l'expérience de l'amour pour un autre être humain» (50). C'est pour cette raison que le personnage d'Angie est important, afin de démontrer comment Faust demeure 
profondément humain puisqu'il peut aimer. Même si Shorty est sous l'emprise de Shadow, il ne perd pas son humanité. Comme Faust est épris de Gretchen, Shorty aime Angie. C'est pour cette raison que Schmidt-Brabant note que «Méphisto . . . ne connaît que le sexe. Il est étranger à l'amour qui ne peut s'épanouir que d'une individualité à l'autre» (50). Pour Méphisto toutes les femmes se valent, étant donné qu'elles n'ont pas d'individualité et qu'elles sont toutes synonymes de jouissance, sans égards aux sentiments. Il en est de même pour Shadow qui vante la beauté de Susie, la veuve de Ketchel, à Shorty, et qui n'est pas contre l'idée que son protégé puisse passer à l'acte avec cette «blanchette» (71). Mais Shorty est outré par cette idée:

SHORTY. Pourquoi cherches-tu toujours le mal derrière tout?

SHADOW. Parce que, derrière tout, il y a le mal. Jeune, jolie, blanche et veuve. Dieu que c'est excitant! Cette femme est incontestablement la forme érotique du diable. Et Dieu sait si je m'y connais en diable.

SHORTY. Tu es odieux, tu salis tout ce que tu touches.

SHADOW. Et toi, tu ne salis peut-être pas tout, toi qui viens farfouiller sous la jupe de la veuve sur la tombe encore fumante du mari? (Shorty s'en va) (71)

Pour Shadow, le fait qu'il puisse ou non exister une liaison entre son boxeur et la veuve ne fait aucune différence, en autant que le plaisir et la jouissance soient réunis. Et dans son immense «bonté», le Diable de Shadow a littéralement manipulé Shorty et sa sœur Angie pour qu'ils entretiennent une relation incestueuse. Pour le Diable, qu'ils soient frère et sœur ne change rien à ses yeux, mais aux yeux de leur entourage et de la société, cette idée dépasse l'entendement car elle est contre nature, strictement interdite, voire criminelle. Shadow pousse donc son emprise encore plus loin que celle de Méphisto. Le couple formé de Shorty et Angie est donc plus outrancier que celui formé de Faust et Gretchen, car il puise sa source au cœur de l'inceste, un tabou fondamental de toute société. Puisque deux membres d'une même famille Noire sont Impliqués, cette situation ne peut que se retourner contre eux, et ce, deux fois plutôt qu'une, entre 
racisme et interdit.

Nous pourrions supposer que Shadow n'est ni plus ni moins qu'un agent provocateur voulant susciter répulsion et dégoût auprès des Blancs qui avaient oublié, jusqu'à un certain point, la couleur de peau du champion de boxe devenu leur héros. Kwahulé réussit ainsi à fusionner plusieurs mythes: d'abord celui du héros Noir champion de boxe, ensuite celui du héros pour qui le rêve américain s'est réalisé en lui permettant d'atteindre la prospérité économique et enfin, celui de Faust et donc de Shorty. Tous deux doivent payer le prix pour avoir accepté que le Mal tire les ficelles, dans le dessein d'apporter la connaissance à Faust et la reconnaissance à Shorty.

Angie tente de sauver son frère des griffes du Diable, mais elle n'y parvient pas. Elle décide de monter de toute pièce une fausse histoire de dopage pour l'obliger à quitter le monde de la boxe après la mort accidentelle de Ketchel. Elle s'organise avec Monsieur Jean, l'entraîneur de Shorty et le seul Blanc ${ }^{31}$ de son entourage, afin que les autorités lui retirent le drott de boxer. Lorsque Shorty a été déchu de son titre, Angie a témoigné au tribunal et confirmé ce que tous savaient sur la nature de sa relation avec son frère. Shadow explique ainsi à Shorty que sa sœur est effectivement en train de perdre la raison:

elle s'est mise à crier que j'en voulais à ton âme, mais que je perdais mon temps, parce que ton âme était passée par son sexe pour se réfugier dans son ventre. Elle s'est déshabillée en plein tribunal et m'a frappé avec sa culotte... Elle me frappait avec cette culotte maculée de sang en criant que j'étais le diable et que j'en voulais à ton âme. ... Mon Dieu, c'était terrible! Les Blancs ont dit qu'elle était... qu'elle avait besoin de repos. Ils l'ont mise dans un hôpital. (81)

L'inceste est clairement affirmé dans ce passage, même si ses propos sont associés au délire d'Angie qui, malgré les apparences, ne fait qu'énoncer la vérité entre elle et son frère et

31 Monsıeur Jean est un Françaıs très respecté de tous Il vouvoıe les gens et ceux-cı le vouvoıent en retour À noter que s'il est Blanc, ıl est également Juıf 
entre son frère et son entraîneur. Ici, l'inceste, le sang et le Diable se rejoignent, puisque Shadow est celui qui a tout orchestré. Là encore, ce sont les Blancs qui jugent de l'état de santé mentale de la femme. Ils estiment qu'elle est devenue complètement folle et décident de l'interner dans une institution psychiatrique.

\subsubsection{Les multiples fonctions des mises en abîme}

Au niveau de la forme de la pièce, le texte de Kwahulé compte de nombreuses mises en abîme puisque Shorty, Angie et Shadow répètent, sous la direction de ce dernier, le Faust de Goethe au sein même de Cette vieille magie noire. Selon Anne Ubersfeld, ce procédé intervient durant les moments où se jouent tout à la fois vérité et illusion théâtrale. Il est également appelé «théâtre dans le théâtre», et survient quand il y a «la présence à l'intérieur de l'espace scénique d'un autre espace, où prend place une représentation théâtrale» (84).

Pour Patrice Pavis, il s'agit d'inclure dans une œuvre «une enclave qui en reproduit certaines propriétés ou similitudes structurales. La réflexion de l'œuvre externe dans l'enclave interne peut-être une image identique, renversée, démultipliée ou approximative» (253). Selon lui, le «théâtre dans le théâtre» constitue la forme dramatique la plus connue de la mise en abîme. Pavis cite même en exemple le Faust de Goethe comme étant l'illustration même d'un «jeu de marionnettes mimant l'action de la pièce et représentant le théâtre du monde» (254).

Georges Forestier reprend également ce thème de théâtre du monde alors que la «pièce intérieure terminée (ou interrompue), l'action principale reprend son cours, modifiée par les répercussions que le contenu de la pièce intérieure a eues sur les personnages («acteurs» aussi bien que «spectateurs») (2:1619).

Il en est ainsi dans Cette vieille magie noire, puisque les personnages de Kwahulé jouent leur rôle dans la pièce mais également des fragments de Faust. Ces passages sont autant d'allerretour entre la réalité afro-américaine et les rêves de cette communauté relativement aux pouvoirs 
de Méphisto et de Shadow. Quant à Shorty, il rêvait d'être comédien mais il est devenu boxeur. En filigrane de cette histoire de boxe se profile celle du théâtre puisqu'il répète, à quelques reprises, des scènes de Faust de Goethe en compagnie de sa sœur et de son manager.

SHADOW. Pareille demande ne m'effraie pas, et je suis prêt à te fournir tous ces trésors. Mon cher ami, le temps est venu de nous plonger à loisir dans les jouissances.

SHORTY. Marché conclu! Je ne me suis pas engagé à la légère: tel que je suis, ne suis-je pas un esclave? Que m'importe de qui? Toi ou un autre! (8)

Cet échange provient effectivement du Faust de Goethe. Koffi Kwahulé a indiqué qu'il souhaitait que les metteurs en scène s'en tiennent à la traduction proposée lorsque Shorty fait du théâtre et qu'il joue Faust, sous la direction de Shadow le diable et en compagnie d'Angie / Gretchen. Nous avons pu établir le rapprochement entre Faust et Shorty, puisqu'ils sont tous deux des esclaves symboliques devant obéir à celui avec qui l'entente a été conclue. Le premier est associé au savoir de l'Homme tandis que le second a trait à la puissance et à la domination du boxeur Noir sur ces adversaires, qu'ils soient Noirs mais surtout Blancs. En s'inspirant du mythe de Faust, Koffi Kwahué rappelle donc la mise en place de systèmes esclavagistes ainsi que la lutte des AfroAméricains pour les droits civiques.

Cette fusion des sources où les répliques du Faust de Goethe rencontrent celles de la pièce de Kwahulé, brouille les pistes de lecture. La seule certitude est que Méphisto et Faust existent explicitement sous les traits de Shadow et de Shorty. Elle démontre effectivement une «rupture dans la continuité dramatique, ou du moins jeu de rôle présenté comme jeu de rôle» (Forestier 2: 1620), puisque le lecteur-spectateur ne sait plus très bien s'il s'agit de la pièce Cette vieille magie noire, d'un match de boxe, des répétions de Faust, des représentations de Faust ou d'un plateau de télévision où un reporter commente le parcours du boxeur. Dans ce dernier exemple, il s'agit d'une autre mise en abîme qui «empêche tout ancrage spatial» (Chalaye, Miroir 39). À cet effet, 
la présence du reporteur télé au début de la pièce permet, grâce à son reportage, de présenter un «document exceptionnel» (Kwahulé 9) sur la vie de Shorty dans lequel tous les personnages sur place sont également mis en scène. En jouant sur les réalités: celles de la pièce de Goethe, celles de Cette vieille magie noire et sur les ruptures des dialogues, le dramaturge ivoirien questionne la place même du Noir au théâtre. Avec ces éléments de distanciation, il rappelle qu'il s'agit d'une représentation théâtrale au sein d'autres représentations théâtrales, créant ainsi un sorte d'inconfort pour le lecteur-spectateur qui doit se questionner sur ce qu'il voit.

Il va même plus loin en demandant si les Noirs ont même droit au théâtre. En effet, il pose directement la question quand Mickey affirme que «faire du théâtre, c'est un truc de vieux... ou de Blanc» (34), comme si cet art ne pouvait être pratiqué par les Noirs. Nous pouvons croire que ce constat est associé à l'absence d'identité vécue par les personnages Noirs, qui ne sont donc pas en mesure de s'approprier d'autres identités que celles héritées des constructions des Blancs. Le Noir est donc évacué puisqu'il ne peut assumer les rôles nécessaires au jeu théâtral. Cette image est au cœur de Peau noire, masques blancs de Frantz Fanon pour qui la conscience de soi de l'homme Noir est une construction du Blanc.

De plus, la réplique de Mickey n'est pas sans rappeler le propre discours de Koffi Kwahulé au sujet de certains éléments du théâtre africain. En effet, dans sa pièce $1+1=1$, il a remis en question le fait qu'il n'y avait jamais eu, auparavant, de pièces écrites pour deux personnages en Côte d'Ivoire parce qu'il s'agissait d'un «truc de Blancs» (Kwahulé, entretien inédit). Avec Cette vieille magie noire, Kwahulé questionne la rencontre entre les théâtres européens et africains et les attentes qui existent de part et d'autres, comme si le produit de cette rencontre devrait entrer dans des cadres prédéterminés et ne pas en déroger.

Shorty est lui-même en train de chercher son identité perdue à travers le théâtre, telle une marionnette manipulée par Shadow qui est à la fois son manager et le metteur en scène de la pièce de théâtre. Shorty est un personnage représenté en tant que marionnette, car il est vidé de 
son identité par l'acte théâtral. De cette manière, Kwahulé met en scène la déconstruction de l'identité individuelle. En ayant recours au théâtre dans le théâtre, il montre que devenir boxeur, pour un homme Noir, signifie être transformé en marionnette, car il est ainsi enfermé dans un stéréotype qui le contraint à agir en conséquence. La société ne lui permet pas de faire des choix et d'être un individu. Cette identité stéréotypée est construite par les discours du monde qui entoure le Noir, comme nous l'avons vu dans Les Nègres de Genet.

Le dénouement de la pièce interroge justement le lecteur-spectateur sur ce qu'il a vu, à savoir l'histoire d'un boxeur ou celle d'une pièce de théâtre portant sur la boxe et le théâtre, car la scène est également le ring. La dernière indication scénographique tout de suite après l'ultime réplique de la pièce est à ce titre fort importante. «Les comédiens en blouse blanche se mettent alors à applaudir tandis que Shorty et Shadow saluent. Puis ils descendent, prennent les autres acteurs par la main et saluent tous le vrai public» (84). Du ring au théâtre puis à l'asile, rien n'est impossible lorsque que Shadow tire les ficelles d'un théâtre grandeur nature.

Si nous tentons de tracer un parallèle entre les pièces Faust et Cette vieille magie noire, il faut d'abord s'intéresser à leur forme. Chez Goethe, l'Urfaust est reconnu pour certains éléments formels, empruntés pour la plupart à la tradition théâtrale. Joël Lefebvre souligne notamment l'«emploi du vers libre . . . langage souvent proche du quotidien, insertion de poèmes chantés, scènes burlesques et parodiques, traits comiques et truculence de Méphistophélès . . . mélange des catégories» (1: 733).

Dans Cette vieille magie noire, il est possible de constater de nombreuses ruptures et éléments de distanciation comme nous l'avons vu: de véritables extraits de Faust joués par les personnages de Kwahulé et qui sont resitués dans l'actualité du spectateur, un reportage journalistique ayant comme effet de présenter les personnages au début de la pièce de Kwahulé, la présence du jazz avec des chants et d'un véritable quartet de jazz, ainsi qu'une mise en abîme qui traite du pacte signé par Shorty et Shadow. Deux jeux parallèles se déroulent à différents 
moments de la pièce. Il y a le Faust de Goethe qui intervient dans un contexte moderne où Shorty et Angie répètent cette pièce sous la direction de Shadow, en vue d'une première théâtrale. Il y a également une mise en abîme où le boxeur et son manager échangent des répliques de Goethe sur le ring. Il s'agit du dialogue qui intervient entre Méphisto et Faust, au moment où ce dernier scelle le pacte avec une goutte de son sang. Mais en fait, le théâtre est tout ce dont rêve Shorty qui aurait préféré monter sur les planches que monter sur le ring. Ce sont finalement les accusations de dopage qui lui permettront de se libérer de la boxe, mais au lieu de se retrouver au théâtre, il sera conduit à l'asile.

Sylvie Chalaye souligne que le théâtre constitue un leurre pour Shorty qui tente ainsi de retrouver son identité perdue. Elle estime que le théâtre est la métaphore du peuple africain. Shorty finit dans un asile, et derrière lui, c'est l'image de tout un peuple psychosé ... qui se profile, d'un peuple qui n'a pas d'autre histoire commune que celle de la colonisation et de l'esclavage, histoire précisément de la dépossession de soi. Les images identitaires que s'est construites le peuple noir ne sont que des leurres: illusions et artifices du «star système», violence et mort de la boxe emportent dans le même tourbillon. Tout se passe comme si ce peuple était voué à la folie, celle d'un être dépossédé de lui-même, auquel on a volé son âme. (Théâtre africain 89-90)

Il est intéressant de constater que, dans la préface de la réédition de la pièce en 2006, Virginie Soubrier pose cette question sur l'identité de la communauté noire et américaine en fonction du «besoin du sacrifice de Shorty» (7), qui rappelle le rituel de mise à mort comme nous le verrons plus en détails dans l'analyse de la pièce Bintou. Nous pouvons croire que ce sacrifice n'est pas seulement l'affaire de Shorty mais également d'Angie, qui elle aussi s'abandonne pour sauver, sans succès, son frère des griffes de Shadow, et qui se retrouve elle aussi internée. Shorty a mauvaise conscience et veut être pardonné, non pas pour s'être associé au Diable, mais bien pour avoir tué Todd Ketchel. Il demande pardon à Susie, la veuve du boxeur, lors de funérailles. 
Elle s'inspire d'un discours biblique pour lui répondre: «Qu'avez-vous fait pour demander pardon, et qui suis-je pour avoir le droit de vous pardonner? Si vous avez mal agi, je sais que Dieu vous a déjà pardonné. N'est-il pas dit qu'il sera beaucoup pardonné à celui qui a beaucoup souffert? Et vous, vous avec beaucoup souffert, Shorty» (Kwahulé 70).

En effet, la souffrance de Shorty n'est pas seulement celle du meurtre / accident de Todd Ketchel mais également celle de tout un peuple. Toujours dans la préface de la réédition de 2006, Virginie Soubrier demande «Comment se fabrique une «mythologie du rêve» chez un peuple qu'on a dépossédé de son Histoire et privé des droits les plus élémentaires?» (7). Koffi Kwahulé semble indiquer que Shorty souhaite connaître ses origines «Noires» afin de retrouver ce rêve qu'il a perdu. Dans sa folie, à la fin de la pièce, le boxeur afro-américain idéalise la vie en Afrique, comme si cette existence était préférable à celle qu'il mène actuellement, celle d'idole déchue, avant l'arrivée du Mal, qu'il s'agisse des colonisateurs, des négriers ou du Diable.

Toutefors, malgré la souffrance, la boxe demeure un sport synonyme d'espoir pour la Communauté et ses enfants, le seul moyen de «reconstruire les rêves, la remettre debout, lui dire que tout est possible» (Kwahulé 39) dans cette «mythologie de l'espoir» (51). Mais selon Negus, cet espoir a un prix. «L'espoir que toute larme noire, que toute goutte de sang noir remontera tôt ou tard, d'une manière ou d'une autre, à la surface de nos consciences. L'espoir que tout crime sera puni» (52). L'espoir dont il est question ici est un espoir de vengeance associé aux souffrances du passé d'esclave et au désir de voir les Blancs et les négriers payer pour l'ensemble des crimes et des humiliations qu'ils ont fait subir au peuple Noir.

Cependant, même si Shorty tente de rompre ses liens avec le Diable, il demeure enchaîné à son passé symbolique d'esclave, alors que la boxe—et la tricherie du Noir sur le Blanc, puisque le Noir ne peut réussir sans tricher-ainsi que le jazz sont les seuls moyens dont il dispose pour tenter de s'affranchir et de retrouver son identité perdue. L'auteur nous montre que la boxe, et le sport en général, demeure l'un des rares domaines où il est possible pour le Noir de se réaliser. 
Toutefois, cette réalisation passe presque exclusivement par le corps: les muscles du sportif, la dextérité du musicien, la voix du chanteur. Koffi Kwahulé semble annoncer un avenir sombre pour les Noirs Américains, qui sont littéralement pris au piège des stéréotypes causés par les constructions que les Blancs leur renvoient depuis toujours. Il démontre que leur seul moyen de s'affranchir et de prouver leur valeur est de tricher. Shorty est donc l'incarnation même de Faust, pris au piège de son identité et du pacte signé avec Shadow qui est, par définition, un assassin et un manipulateur.

Dans cette réécriture du mythe de Faust, Kwahulé permet d'entrevoir l'ouverture d'un certain espace, si infime soit-il, puisqu'il convoque et fusionne les mythologies du rêve et de l'espoir, malgré l'incertitude du destin qui guette Shorty. Faust est un révélateur parfois violent de cette probable manière de survivre à travers cette métaphore d'un peuple toujours dominé. Son pouvoir permet d'ébranler le rapport entre Noirs et Blancs. En effet, Kwahulé réussit à créer une nouvelle mythologie d'avant la colonisation, alors qu'une infinité de possibilités préexistait. Il s'agit peut-être là du véritable sens de Cette vieille magie noire, où il est justement possible d'opérer des changements avec l'aide du Diable qui manıpule les hommes et d'un dramaturge qui en fait tout autant. Si le Faust de Goethe s'attardait à la question de la connaissance, celui de Kwahulé marque plutôt le désir d'opérer des changements dans cette relation forgée depuis toujours et qui unit le Blanc et le Noir. On pourrait même dire qu'il met en scène un renversement des valeurs du Bien et du Mal en accordant à Méphisto une allure positive, soit celle du dandy intellectuel qui est également très cool.

Si l'action de Cette veille magie noire se situait en Amérique, celle de Bintou a bel et bien lieu en France. Là encore, il est question de cette identité attribuée au Noir et du désir de ne plus, justement, occuper la place qui est réservée aux Noirs. Bintou, cette femme-enfant, est une victime sacrificielle qui doit passer par le couteau de l'exciseuse afin qu'une nouvelle mythologie puisse s'amorcer. Bintou incarne le refus de cette identité construite de toute pièce à laquelle on 
veut la soumettre. Contrairement au fardeau imposé par la société Blanche et à laquelle le Noir ne pouvait déroger dans Cette veille magie noire, Bintou entend bien faire des choix personnels, quitte à se mettre à dos toute sa famille. En ce sens, Bintou incarne des valeurs individuelles d'où émergent la création d'une nouvelle mythologie culturelle, contrairement à Shorty et à Shadow qui ne peuvent exister hors du collectif.

\subsection{Bintou et le sacrifice expiatoire}

Bintou est une pièce qui se situe dans un contexte contemporain et qui évoque la question de l'immigration en France. Le point de vue de Koffi Kwahué se situe de l'intérieur, c'est-à-dire qu'il traite de cette question à travers la vie d'une famille africaine qui a émigré en France. Avec cette pièce, il continue sa réécriture des mythes en situant l'évolution de Bintou, la protagoniste principale dans le contexte d'un rite de passage. En effet, Bintou devient la victıme sacrificielle de la mythologie chrétienne, et sa mise à mort se réalise dans le cadre d'une cérémonie traditionnelle africaine que constitue l'excision. Le rite de l'excision est une pratique actuellement interdite en France ainsı que dans de nombreux pays occidentaux et également africains, dont le Burkina Faso, le Sénégal, le Ghana, l'Érythrée et l'Ouganda, même si, dans les faits, sa pratique n'a pas cessé.

Dans Je vois Satan tomber comme l'éclair, René Girard rappelle que «Les rites dits de passage ou d'initiations sont fondés, comme tous les rites, sur le sacrifice, sur l'idée que tout changement radical est une espèce de résurrection enracinée dans la mort qui la précède et qui peut seule remettre en branle la puissance vitale» (145).

La pièce de Koffi Kwahulé est une tragédie inspirée de la tradition aristotélicienne où Aristote considérait que le mal n'est pas dans le monde. À partir des principes qui définissent la structure tragique, le mal est le résultat de la faiblesse fatale du héros, appelée «hamartia», (Pavis 424) et qui le mène à sa perte. Dans le cas de Bintou, la révolte de l'héroïne et son refus 
d'accepter les conditions de la culture de ses parents se traduiront par sa mort.

Dans le Dictionnaire encyclopédique du Théâtre, Suzanne Said écrit que: «Ce qui est au cour de la tragédie, ce sont les rapports que l'individu entretient avec les groupes, famille ou cité, auxquels ils appartiennent, ainsi que le problème capital des rapports du public et du privé» (2: 1646). De plus, dans le théâtre contemporain, elle décrit ainsi la tragédie où l'on «tend à mettre l'accent sur la dimension religieuse de la tragédie grecque qui met en scène des sacrifices humains, fait grand usage des métaphores sacrificielles et intègre des éléments rituels comme la supplication et la prière» (2: 1647) et où le chœur joue un rôle central.

Ces définitions correspondent au texte de Koffi Kwahulé. La pièce est une tragédie, en ce sens qu'elle se termine par la mort de Bintou. La fable raconte la vie de Bintou, une jeune fille de 13 ans d'origine africaine - mais née en France--et qui est à la tête d'un gang de rue constitué de garçons beaucoup plus âgés qu'elle. Elle défie toute forme d'autorité. Les deux choses les plus importantes pour elle sont sa bande, dont les membres veulent lui plaire à tout prix, et sa danse du ventre, qu'elle répète inlassablement. Elle a complètement rejeté sa famille et les valeurs traditionnelles qu'elle veut lui imposer. Son père est devenu invisible après avoir perdu son travail, abdiquant du même coup son autorité. Sa mère fait tout ce qu'elle peut pour garder sa fille dans le droit chemin, mais n'y parvient pas. De plus, son oncle et sa tante voudraient lui imposer leurs propres lois, celles de la communauté africaine, étant donné que sa mère en est incapable. Bintou sait qu'elle va mourir sous peu mais cette perspective ne l'inquiète pas. Elle s'est construite son propre univers qu'elle dirige à sa guise. Son oncle parvient à la retrouver après avoir payé une bande rivale pour l'enlever et la ramener dans sa famille pour qu'elle se fasse exciser. Mais Bintou meurt entre les mains de l'exciseuse.

La pièce illustre comment plusieurs cultures s'entrechoquent au sein de cette famille immigrante. La famille de Bintou tient toutefois à ce que la jeune fille se fasse exciser afin de la purifier et de mettre fin au chaos qui l'entoure, dans cette société urbaine de l'Europe 
postmoderne. Il s'agit plutôt de la rencontre brutale entre tradition et modernité et d'un dialogue de sourds qui s'établit sans communication possible autour de la question du maintien des traditions. Tous les thèmes, bien qu'ils touchent de près à l'Afrique, parlent également de nouvelles réalités pour les immigrants qui s'installent dans un autre pays, telles qu'elles ont peutêtre été vues ou vécues par le dramaturge qui réside en France depuis le début des années 1980.

La pièce, qui est structurée comme un Calvaire, s'articule autour de sept volets qui commémorent la Passion du Christ. Il s'agit de la marche de Bintou vers son anéantissement qui est, en fait, une représentation du rituel chrétien du Christ sur le chemin de croix, où chacune des 14 stations marquent une étape de la condamnation de Jésus: sa marche et ses chutes avec la croix, ses rencontres, ses vêtements qui lui sont retirés, sa crucifixion, sa mort, la remise de son corps à sa mère et sa mise au tombeau. Bintou connaîtra un parcours semblable, à la différence que la crucifixion est ici remplacée par l'excision.

Le premier tableau intitulé Tentatıons se déroule là où vit la famille de la jeune femme, «dans un modeste intérieur d'une famille d'immigrés noirs africains» (5), avec sa mère qui fait des ménages et son père invisible, caché en permanence dans une pièce de la maison. Sa tante Rokia déteste Bintou car elle est jalouse d'elle. Drissa est un «oncle hypocrite qui dissimule ses désirs incestueux sous de faux préceptes moraux» (Chalaye, Miroir 37). Et bien que l'oncle et la tante n'habitent pas avec Bintou et ses parents, ils sont très présents à la maison.

Le second tableau intitulé Jazz présente l'entourage masculin de Bintou et de sa bande avec Manu, Blackout et Kelkhal, et également P'tit Jean, un drogué, qui aspire, lui aussi, à devenir un membre du gang des Lycaons ${ }^{32}$ que dirige la jeune fille.

Fils est le nom du troisième tableau qui met en scène P'tit Jean et sa mère. Le jeune homme admire Bintou et affirme qu'elle est une sainte. Il est toutefois en manque et s'en prend à 
sa mère, car elle n'a plus d'argent à lui donner afin qu'il se procure de la drogue.

Le tableau suivant s'intitule $U s$ et renvoie aux us et coutumes de la tradition africaine. En français, «us» signifie littéralement «nous» mais peut également vouloir dire US, pour United States ou États-Unis. Le «nous» est celui de la famille de Bintou qui veut qu'elle revienne dans le giron familial. Ce tableau illustre la rencontre entre la mère, la tante et l'oncle de Bintou et Moussoba, une vieille femme exciseuse. La mère voudrait envoyer sa fille en Afrique. Mais ce voyage n'est qu'un prétexte, car la famille souhaite secrètement qu'elle y soit excisée. Bintou refuse l'offre de voyage. C'est pourquoi elle devra donc être excisée pieds et poings liés à la maison, pour chasser le démon de la luxure qui l'habite.

Le segment suivant s'appelle Repentance. C'est à ce moment que l'oncle remet de l'argent et de la drogue à P'tit Jean afin qu'avec ses amis Pitbulls, ils enlèvent Bintou pour la ramener à la maison. Le tableau 6, Gangsta Rap-t, se déroule dans le bar de Nenesse. Tandis que les Lycaons affrontent P'tit Jean et ses Pittbulls lors d'une bagarre générale, P'tit Jean en profite pour enlever Bintou. Le titre de ce tableau possède une double signification. Il met en lumière un certain style musical d'abord créé par les jeunes Noirs Américains des ghettos, le «Gangsta rap», dont nous analyserons la portée un peu plus tard dans ce chapitre. L'autre partie du titre évoque le rapt, soit l'enlèvement de Bintou.

Intitulé Viol, le dernier tableau est la cérémonie d'excision qui tourne au meurtre. Avant de mourir, Bintou s'imagine qu'il s'agit de la cérémonie de son mariage avec les trois jeunes de sa bande de Lycaons. Nous voyons ainsi comment Bintou est celle qui est choisie afin de servir au sacrifice, comme l'illustre le calvaire d'une figure christique.

Comme nous l'avons vu, le modèle mythologique qui est transformé par la pièce est le rituel du chemin de croix de Bintou. Dès le départ, elle est condamnée à être crucifiée / excisée selon les traditions et croyance de sa famille. À l'image de Jésus qui est chargé de sa croix, Bintou porte le fardeau de sa mort toute proche, mais celle-ci, contrairement à Jésus, ne tombe 
pas à trois reprises. Elle rencontre ses trois Lycaons, P'tit Jean et sa propre mère qui tente de l'envoyer «au pays». Puis elle est enlevée par P'tit Jean et remise à sa famille. C'est à ce moment que Bintou est dépouillée de ses vêtements par son oncle qui la frappe violemment jusqu'à ce qu'elle perde connaissance. On lui écarte alors les jambes pour l'exciser puis elle meurt. Jésus avait lui aussi été dépouillé de ses vêtements avant d'être cloué sur la croix et de mourir. Le corps de Jésus avait été détaché de la croix, remis à sa mère et mis au tombeau. Ce sont les Lycaons qui prennent le corps de Bintou à bout de bras avant de sortir. Puis Drissa, suivant les conseils de l'exciseuse, creuse une tombe à l'intérieur de la maison, là où «Personne n'aura l'idée de fouiller» (46).

Nous voyons comment Bintou a favorisé la création de sa communauté de disciples et comment toutes les expériences négatives qu'elle a subies avec sa famille, son oncle, la trahison de P'tıt Jean, l'excision et sa mise à mort symbolisent les étapes de son propre Calvaire dans le monde de l'immigration.

Koffi Kwahulé met justement en scène cette société de l'immigration en France. En présentant d'abord les membres de la bande par leur surnom ou par leur pseudonyme, il indique ensuite l'origine de chacun d'entre eux (africaine, européenne et maghrébine), leur âge, leur véritable nom et leur rôle. Il s'agit d'un élément central dans la pièce, tout autant que la musique, puisqu'il est question d'immigration certes mais également des conflits engendrés par ces rencontres entre des personnages d'origines ethniques différentes. Nous tenons à souligner ces références qui renvoient à la couleur de la peau ou aux origines ethniques dans Bintou et Cette vleille magie noire, puisqu'elles ont complètement disparues des pièces écrites plus tard dans sa carrière.

Dans Bintou, les personnages se retrouvent dans un «lieu clos», la cité dans laquelle les policiers ne se donnent même plus la peine de patrouiller. Il s'agit d'une zone qui n'est plus sous le contrôle de l'État et qui, jugée trop dangereuse, a été abandonnée à elle-même. C'est là où la 
tragédie se joue dans un espace à l'écart de la société, où les représentants officiels de

l'administration municipale n'interviennent plus et où les habitants établissent un régime parallèle ou périphérique qui les isolent encore davantage du «centre».

\subsubsection{Le rôle du chœur dans la tragédie}

Nous avons vu que Bintou pourrait être désignée comme une tragédie si nous tenions compte des éléments qui caractérisent le théâtre grec, dont l'une des conventions est le chœur. Il est intéressant de noter la manière dont Kwahulé transpose le chœur grec à la scène contemporaine, où il lui confère de nouvelles fonctions scéniques et dramaturgiques. Ici, le chœur est composé de trois adolescentes. Dans la pièce, le chœur, en tant que convention théâtrale, fonctionne de plusieurs façons. Il permet notamment d'élever le personnage principal en héros mythique, car le chant du chœur est icı synonyme d'un chant funèbre qui magnifie le sacrifice de Bintou par sa famille. Le chœur est également la voix narrative qui relate le calvaire de la jeune fille.

Dans le premier tableau, le chœur intervient d'entrée de jeu au moment où Bintou entre en scène. Le chœur la précède et porte un miroir et une trousse à maquillage. Il récite d'une seule voix plusieurs éléments qui situent le contexte dans lequel Bintou évolue-la jeune fille est une fleur sauvage dans une cité où la loi n'a plus d'emprise-et qui décrivent l'univers de l'adolescente articulé autour de ses Lycaons, de sa danse du ventre et de son couteau, un univers où la vie et la mort se côtoient au quotidien. Le chœur énonce également l'autre nom de Bintou, celui de Samiagamal $^{33}$ et compare sa manière de danser à celle d'une déesse.

Le chœur prévient également Bintou des dangers qui la guettent: «Mais voici venir la

33. Samıagamal est en fait Samıa Gamal (1924-1994), l'une des grandes figures de la danse orientale également appelée danse du ventre dans la langue populaire Elle était d'orıgine égyptıenne Elle incarnait le charme, la beauté et la volupté Elle a rendu la danse du ventre plus respectable Elle y a ajouté les chaussures à talons hauts, le voıle, une certaine forme d'ımprovisatıon et des éléments provenant de d'autres types de danse Koffi Kwahulé a déjà publié . Et son petit amı l'appelait Samıagamal dans Brèves d'aılleurs Actes-Sud Papıers, 1997 Cette pièce est ensulte devenue Bıntou 
famille / Mais voici l'ombre de la dame-au-couteau / Mais voici venue l'heure des grandes résolutions» (4). Durant cette première intervention du chœur Bintou reste immobile, et l'auteur précise que le temps est suspendu. Ainsi, le chœur est un narrateur omniscient, selon les principes de la narratologie. Il a une fonction annonciatrice et prophétique, puisqu'il connaît la vie et le destin de Bintou. En même temps, le chœur parle de Bintou à la troisième personne tout en employant le «je», montrant ainsi la fusion Bintou-chœur, comme si le chœur, sachant à l'avance ce que le sort lui réserve, était aussi la conscience de la jeune fille. Selon Virginie Soubrier, «Le «chœur africain» recouvre ... les fonctions dramaturgiques traditionnelles répertoriées du chœur grec: comme dans les textes antiques, il a une complicité étroite avec le héros, mise en valeur par une énonciation ambiguë, intégrant dans le discours du chœur la parole de Bintou» (Dionysos 142).

Le chœur revient au moment où Bıntou se maquille et que son oncle entre dans sa chambre pour lui interdire de sortir de la maison. La jeune fille, pleine de sous-entendus, lui dit qu'il n'est pas toujours aussi intransigeant et lui rappelle la dernière fois qu'il lui a rendu visite dans sa chambre. Le chœur la rejoint sous le fassceau de lumière et l'aide à se maquiller, puis à se préparer. Il participe à l'action en tant qu'ami fidèle de Bintou. Il est également témoin de la conversation entre Bintou et son oncle, alors que ce dernier essaie de séduire la jeune fille. Il lui fait remarquer qu'elle ne porte ni soutien-gorge ni culotte, tout en lui glissant la main sur les fesses. Elle l'arrête en le menaçant avec son couteau. Le chœur et l'oncle quittent le cercle lumineux. Nous voyons qu'ici le chœur se transforme en ange gardien, une présence transcendante qui protège la jeune fille et qui la suit lorsqu'elle quitte la maison. Si le chœur de Koffi Kwahulé renvoie à la tradition théâtrale grecque, le dramaturge met également en lumière une autre tradition: celle où un oncle peut agir à titre de père. Ce tableau intitulé Tentations démontre le côté pervers de cette tradition puisque l'oncle Drissa, qui désire sa nièce, est excité par sa présence. Sous des faux airs de protecteur, il se permet des gestes et des paroles qui vont 
bien au-delà de ce qui est attendu de son rôle de père. De nouveau, Bintou incarne, aux yeux de son oncle, le désir de la chair, malgré qu'il soit à la fois son oncle et sa figure paternelle. Il s'agit une fois de plus d'une subversion des catégories de la mythologie chrétienne dans laquelle la chair est synonyme de péché et d'inceste. Koffi Kwahulé rétablit les rapports de chair en inscrivant le corps dans un rapport de manipulation et de domination.

Le chœur revient dans le tableau suivant lorsque P'tit Jean se fait passer à tabac au son d'une «rap ravageur», après avoir démontré qu'il n'était pas digne de devenir membre de la bande, refusant de tuer quelqu'un sur commande. Il ne peut donc pas joindre les rangs des Lycaons. Puis les voix de Bintou et du chœur alternent en rappelant à P'tit Jean qu'il «deale» sa vie et celle du monde. Virginie Soubrier parle d'un «chant partagé entre Bintou et le chœur qui vient amplifier l'action» (142). Elle trace également un parallèle entre le chœur grec et celui présenté dans Bıntou: «Ce dernier devient ainsi, comme dans les tragédies grecques, un motif théâtral capable de fabriquer du rythme, tant par le langage poétique qui lui est réservé que par ses apparitions dans l'architecture de la pièce. Il est ici un élément organique du théâtral, à l'instar des tragédies athéniennes . . . dans lesquels parties chantées et parties dialoguées éta1ent intimement liées» (142).

Le chœur ne revient qu'au dernier tableau de la pièce quand l'oncle gifle Bintou. De nouveau, le chœur se transforme en ange gardien puisqu'il accourt au moment du coup. Quand Moussoba pratique l'excision, le chœur devient témoin du «crime» et il décrit tout ce qui se passe. En effet, Koffi Kwahulé indique dans les didascalies de la pièce que «personne n'exécute ce que disent les répliques» (43). Toujours selon Virginie Soubrier, «le chœur, dans la scène finale de l'excision, joue le rôle de Messager de la tragédie grecque» (144). Le chœur observe, devenant témoin et voyeur. Il voit tout et décrit tout ce qui arrive à Bintou en employant la troisième personne, «elle», mais également le «je» lorsque le chœur dit: «Aussitôt je sentis le froid baiser / De la lame sur la crête de mon secret» (Kwahulé, Bintou 44). Puis, l'un des membres du chœur se 
met à hurler, ce qui n'est pas sans rappeler une autre convention de la tragédie grecque dans laquelle, cris et hurlements sont présents. Tous ces éléments associés au chœur démontrent une fois de plus la construction de Bintou en tant qu'héroïne mythique, comme l'explique René Girard.

Dans l'ouvrage Dramaturgies africaines en dix parcours, Sylvie Chalaye compare Bintou à une «madone d'Apocalypse» et l'excision à une «crucifixion» (23). Bien que notre étude s'intéresse seulement aux textes des pièces de Koffi Kwahulé, nous ne pouvons pas passer sous silence la mise en scène de Laëtitia Guédon, telle qu'elle a été présentée en Avignon en 2009, et qui a été captée dans le but d'en faire un film réalisé par Greg Germain. La pièce se termine justement sur la mort de Bintou qui, tel un Christ en croix, a été sacrifiée, mais dont la mort ne sauvera personne.

Dans le dernier tableau, après l'excision, Bintou se redresse comme dans un rêve pour accueillir ses Lycaons et P'tit Jean. C'est à ce moment qu'elle découvre qu'elle se vide de son sang. Au moment où elle s'écroule, le chœur retrouve sa fonction traditionnelle en entonnant un chant funéraire oriental. Après sa mort, le chœur se transforme en personnage visible par l'oncle et devient témoin direct des événements. Le chœur surprend l'oncle Drissa qui vient de creuser la tombe de Bintou. Silencieux, le chœur entoure l'oncle qui est pris de panique, persuadé que les trois adolescentes sont des amies de Bintou. Il leur explique qu'elle est retournée au pays, mais le chœur demeure silencieux. Drissa sort mais il est toujours suivi par le chœur qui assume une fonction semblable à la conscience de l'héroïne. Il est présent physiquement mais ne parle plus, réduit au silence par la mort de Bintou. Dès lors, il hantera la famille qui devra s'abstenir de tout signe de deuil afin de ne pas éveiller les soupçons. En conséquence, la famille devra mentir à son entourage et affirmer que la jeune fille, qui a finalement accepté l'offre de voyage, est retournée «au pays». 


\subsubsection{La perspective de l'immigration}

Bintou constitue une synthèse de l'approche de Koffi Kwahulé par rapport aux questions d'identité africaine. Étant donné la manière dont il a recours aux conventions de la tragédie au théâtre, et la façon dont il représente cette jeune fille de la rue qui symbolise la révolte de tous les jeunes de cette mouvance, nous avons l'impression que la pièce est une sorte de texte fondateur d'une nouvelle mythologie urbaine de l'immigration. Le thème principal de Bintou est donc l'identité et la transformation identitaire dans le contexte de l'immigration. L'action se déroule en milieu urbain, dans la France de l'immigration sans toutefois que l'auteur ne précise le lieu exact. Nous pouvons toutefois déduire qu'il s'agit d'une cité de banlieue où les jeunes, souvent issus de l'immigration d'Afrique de l'Ouest et d'Afrique du Nord, sont confrontés à un monde qui leur laisse peu de place, dans cette société française contemporaine également synonyme de la France coloniale d'autrefois. À ce chapitre, nous pouvons donc voir un rapprochement certain entre les émeutes survenues en France à l'automne 2005 qui, à la suite du décès de deux adolescents à Clichy-sous-Bois, ont opposé des jeunes aux forces policières. La pièce Bintou, publiée en 2003, et surtout Et son pettt ami l'appelait Samiagamal, écrite en 1996 et publiée l'année suivante, montre que Koffi Kwahulé était déjà extrêmement sensibilisé à la gravité de la situation ansı qu'aux dangers que cette exclusion de toute une partie de la jeunesse pouvait représenter pour la société française. Il a observé la société et a compris les tensions qui étaient en train de se cristaliser dans les cités des banlieues.

Nous pouvons y voir un rapprochement avec le film La Haine du réalisateur Mathieu Kassovitz, un long-métrage qui avait choqué et bouleversé la société française lors de sa sortie, en 1995. C'est grâce à ce film, entre autres, que la France a compris les changements importants qui étaient en train de s'opérer. En effet, la population n'était plus uniquement Blanche et une coupure s'était produite entre les gens des cités et ceux de la ville. Les habitants des cités ne pouvaient pas trouver leur place au sein de la culture française urbaine traditionnelle. Dans $L a$ 
Haine, il y a également trois jeunes amis qui ont tous en commun le fait de détester les forces policières. Le trio est formé d'un Juif, d'un Maghrébin et d'un Noir. Le film est inspiré d'un fait réel, soit la mort de Makome M'Bowolé, un jeune zaïrois de 17 ans abattu par un policier lors de son interrogatoire dans un commissariat de Paris, en avril 1993. Des émeutes opposant de jeunes gens aux forces policières avaient alors éclaté et duré plusieurs jours.

Bintou est une pièce hybride qui réitère les références africaines dans un milieu occidental transformé par la présence de ces nouveaux immigrants. Ce caractère hybride se retrouve tant dans la forme que dans la thématique qui est abordée. Par exemple, lorsque la mère de Bintou lui demande si elle souhaite «aller au pays» (31) pour connaître le reste de ses proches et découvrir les racines de sa famille, la réponse de la jeune fille ne se fait pas attendre. «Mais mon pays c'est ici maman. C'est la cité, le quartier, le béton, mes mecs... mes "Lycaons", comme dit tante Rokia. C'est icı que je suis née et je n'ai pas envie de connaître autre chose. Ça me suffit» (32). Bintou ne veut pas aller en Afrique parce qu'elle estime ne pas être prête. Peut-être craint-elle ce retour aux sources ancestrales où elle risque de se sentir telle une étrangère visitant un pays lointain, jadis colonie française et actuel pays du Tiers-Monde qu'elle ne connaît pas. Sa connaissance du pays, dont le nom n'est pas précisé, provient de l'histoire de ses parents, de fables et de récits très éloignés d'elle et de souvenirs d'une Afrique qui n'existe plus et auxquels Bintou ne peut s'associer. «Je serais prête à prendre des vacances partout... en Chine, en Amérique, au Brésil, même à Béthune... mais en Afrique, ça non. En tout cas pas maintenant; je ne suis pas encore prête pour l'Afrique)» (33).

La perspective de Bintou—qui se voit comme une Française et non pas comme une Africaine et qui, de surcroît, transgresse les valeurs traditionnelles ancestrales ainsi que tous ses codes sexuels et moraux - amène une déstabilisation des frontières identitaires. En rejetant les valeurs de ses parents, elle se condamne à une double exclusion, celle de sa propre famille et celle de la communauté immigrante africaine de ses parents. Elle provoque donc une redéfinition 
des pouvoirs entre deux univers - univers urbain et univers de l'immigration-mais aussi entre la famille traditionnelle africaine et cette jeune «Française» qui impose le respect à sa bande. Ainsi, la famille traditionnelle africaine est complètement dépassée par les actes et les paroles de Bintou, une fillette de 13 ans dont la féminité est hypersexualisée et qui agit comme un homme. Patronne d'un gang de rue qui carbure à la haine, à la colère et à la violence, elle s'est de plus mise en ménage avec un Blanc de 18 ans. Elle rejette toute forme d'autorité, qu'elle soit familiale (la loi du père absent et de la famille), civile (la loi et la cité), scolaire (l'école) ou religieuse. Sa famille clame qu'elle a le péché en elle, qu'elle est une bonne à rien, qu'elle n'aspire qu'à blasphémer et qu'elle est une dépravée. Ainsi, le père qui ne joue plus son rôle d'autorité, la mère qui doit faire des ménages pour gagner un peu d'argent et la fille qui refuse toute trace d'un passé et d'une tradition africaine qu'elle ne connaît pas sont tous des exemples des conséquences de l'immigration.

En fait, sa bande de Lycaons qu'elle dirige au doigt et à l'œil devient la véritable famille de Bintou. Elle est formée de trois jeunes hommes plus âgés qu'elle. Il s'agit de la culture des jeunes immigrants, Noirs ou Maghrébins, en France associée au Gangsta Rap ${ }^{34}$ des Noirs américains. À travers ses codes et ses règles, la bande devient la nouvelle famille dont les

34 L'expression Gangsta Rap est la fusıon de Gangster en langue populaıre et de Rap, une façon rythmée de dıre les parole en parlant davantage qu'en chantant. Il s'agit d'un style musical qui, par la suite, s'est transformé en phénomène social. Le Gangsta Rap a surtout émergé à la fin des années 1980, sur la Côte Ouest des États-Unıs Ses chanteurs et groupes étaient d'abord des Noirs Américains Ils font l'apologie de la réussite sociale et financière, du mode de vie de gangster, de la violence, du commerce de la drogue, du proxénétısme, de la misogynıe, de la possession et de l'usage d'armes à feu, du meurtre, de toutes actıvités ıllıcites, aınsı que de la haine de la polıce, des homosexuels et parfors des Blancs Ils traitent également du racisme, de la vie dans les ghettos, de la violence famılıale et de la pauvreté Ces chanteurs à l'attıtude machıste et domınatrıce sont souvent accompagnés de jeunes femmes légèrement vêtues et volontaırement soumıses, quı adorent s'exhıber aux côtés de leurs hommes dans des voitures de luxe La domınation des hommes (the boss) est très importante, notamment à l'égard des femmes qui sont consıdérées comme des objets sexuels (the bitch est une femme assolffée d'argent) Certaines femmes réussissent tout de même à se faıre un nom du côté du rap comme Lil' Kım, Eve et Mıssy Elhott

Dans les années 1990, s'ensuit une véritable guerre entre les rappeurs de la Côte Ouest et de la Côte Est qui se soldera par les assassinats de Tupac Shakur et de The Notorious B I.G en 1996-1997 Le Gangsta Rap fait partie de la grande culture hıp-hop quı englobe une façon d'être et de parler, maıs aussı de s'identıfier et de se vêtır (avec des marques de mode spécifiques) et de se parer de nombreux bijoux clınquants (le Blıng-blıng) La majorité des rappeurs adoptent des pseudonymes comme noms d'artistes Par la suite, des Blancs se sont joints au mouvement, tel que Marshall Bruce Mathers III, mieux connu sous le nom d'Emınem Le Gangsta Rap demeure aujourd'huı très popularre et ses artıstes, bien que souvent controversés, sont présents sur toutes les tribunes musicales popularre aınsı qu'à la télévision (MTV), au cınéma, dans les magazınes et les publıcités. 
membres se jurent fidélité, au détriment des liens qui les unissent à leurs familles d'origine. Les trois jeunes hommes se décrivent comme des frères, des «brothers» (18), et tous sont rebaptisés d'un nouveau nom par Bintou qui, ainsi, désire marquer leur nouvelle appartenance. Cette dernière décide également de changer son prénom pour Samiagamal. Emmanuel le Français devient Manu, son petit ami, qu'elle appelle aussi son «Envoûtement». Kader le Maghrébin devint Kelkhal et Oukoumé l'Africain se transforme en Blackout.

Les Lycaons empruntent plusieurs éléments au Gangsta Rap et aux images des chanteurs de la culture populaire Noire des jeunes des États-Unis telles la possession d'une arme à feu, d'un couteau à cran d'arrêt, la valorisation du meurtre, le fait d'avoir «bouté la flicaille hors du quartier» (15), le passage à tabac d'un jeune en guise de rite d'initiation, le commerce de la drogue, le vol de voitures, l'incendie d'une école, rouler en sens inverse de la circulation et l'homophobie. Les jeunes hommes doivent impressionner Bintou en posant des gestes qu'elle approuvera et qui leur confèrera une plus grande valeur à ses yeux. Blackout a tué, de sang froid, un homme qui reluquait Bintou et qui l'avait traité «d'enculé» (20). Ayant éprouvé une grande fierté à la suite du meurtre, la jeune fille a alors rebaptisé Okoumé du nom de Blackout. Pour Manu, ce meurtre constitue le «top» (20) et il aimerait bien à son tour commettre un acte tout aussi grandiose. «En plus, ce mec, il l'a buté pour Samiagamal. Et ça, c'est mortel, on peut rien contre. The dream. Alors je fouille, je cherche ce que je pourrais offrir à Samiagamal qui soit plus géant que ça» (20).

C'est pourquoi à la fin de la pièce, au moment de la mort de Bintou, Manu lui apporte un cadeau de mariage à la hauteur de ses attentes: un képi de policier qu'elle qualifie de «scalp». Il s'agit d'une autre référence à la culture américaine. En effet, cette pratique amérindienne consistait à retirer le scalp des ennemis, les guerriers rapportant ce trophée auprès des leurs en signe de victoire. Dans Bintou, il s'agit du geste d'amour suprême pour Manu, car, en abattant un policier, il accomplit le plus grand exploit qui soit aux yeux de la jeune fille. 
Toutefois, deux éléments importants différencient la bande de Bintou des adeptes du Gangsta Rap où les rappers s'entourent de jeunes femmes soumises, souvent appelées «bitch» ou «ho» (putain). D'abord, le renversement des rôles est primordial: ici, les hommes sont dirigés par une jeune fille bien plus jeune qu'eux. La domination n'est pas masculine mais bien féminine et ce sont les hommes, et non les femmes, qui suivent le chef. La jeune fille détient le pouvoir de décision puisque «Bintou ne suit personne, c'est toi qui suit Bintou» (16). S'il n'y a pas d'échanges de propos sexistes entre les trois garçons et Bintou, c'est parce qu'elle contrôle tout. Elle s'incarne en une présence plus grande que la vie, si éloignée de la réalité qui les entoure, que ce personnage devient rapidement une figure mythique.

Quant à la drogue, Bintou en interdit formellement sa consommation, bien qu'elle accepte son commerce sous forme de «deal». Au contact de la jeune fille, Manu a cessé de prendre du crack, car il devait choisir entre Bıntou et la drogue. Celle-ci sert le même avertissement à P'tit Jean, le junkie, qui voudrait faire partie du groupe afin de ne plus y toucher, lui non plus, pour éviter de se faire sauter la cervelle. Le «rap» de la drogue explique le point de vue de Bintou: «Deale à leurs femmes stressées / Deale à leurs maris surmenés / . . / Deale devant les écoles de leurs enfants / Deale devant les asiles de leur vieux parents / Deale devant les asiles de leurs vieux parents / . . / Deale puisqu'ils dealent l'amour» (25).

À la fois crainte et adulée par ses hommes, Bintou est une idole, synonyme de légende et d'érotisme dans la cité. La légende prétend que les Lycaons se donnaient rendez-vous dans un bar pour se détendre à la suite de leurs aventures. «lls fermaient portes et fenêtres et, sur une musique orientale composée par le diable, Samiagamal livrait, comme une offrande, son corps nu aux corps tendus par le désir de ses Lycaons. Elle leur faisait l'amour à perdre haleine, jusqu'à ce que le dernier Lycaon s'écroule à ses pieds, vidé.... Or, les Lycaons n'étaient en tout et pour tout que trois» (16).

La domination de Bintou est également d'ordre sexuel, contrairement aux images 
véhiculées dans les clips de hip-hop dans lesquels l'homme fraye avec des hordes de jeunes femmes soumises. Bintou incarne le pouvoir sexuel où les rôles sexuels sont subvertis. Elle le sait et s'en sert pour combler ses Lycaons. Elle refuse toutefois de satisfaire les désirs de son oncle incestueux qu'elle repousse à maintes reprises, parce qu'il est son oncle mais surtout parce qu'il la désire et non pas l'inverse.

Bintou porte toujours des jupes trop courtes et jamais de culottes ni de soutiens-gorge, ce qui rend les hommes fous d'elle. Blackout a conduit à toute vitesse une voiture volée en sens inverse, alors qu'elle a glissé sa jambe entre les cuisses du chauffeur afin d'appuyer elle-même sur l'accélérateur. «Crois-tu qu'il y ait une façon plus excitante de mourir?»(17). Kelkhal a vu Bintou couchée sur le dos, les jambes repliées et n'a pu s'empêcher de lui embrasser le nombril. À ce moment, il voulait savoir pourquoi elle ne venait plus à la maison. «Les hommes ne sont que les hommes, Kelkhal, et je ne voulais pas que ta mère soit malheureuse» (19). Nous pouvons supposer qu'il s'est produit quelque chose entre Bintou et le père de Kelkhal, étant donné que celui-ci avait comparé la jeune fille à Samia Gamal, une comparaison qu'il n'avait même jamais osé faire à l'époque où sa femme était danseuse du ventre.

Bintou possède donc un très grand pouvoir sexuel qui s'approche du mythe puisque ce qu'elle symbolise est plus grand que la réalité. Son attitude à l'égard de la sexualité et du désir tend au renversement des rôles puisqu'elle se donne uniquement à ceux qu'elle choisit, sans tenir compte des règles et des valeurs de la famille, de la société traditionnelle africaine ou même française. Elle n'obéit qu'aux seuls impératifs qu'elle a personnellement mis en place au sein de sa bande, le seul endroit où son pouvoir n'est jamais remis en cause. Il existe même un certain flou concernant ses relations avec les membres des Lycaons, comme si les mœurs qu'ils entretiennent devaient être gardées sous silence. Bintou a reconstruit son monde sur le modèle d'une société secrète qui fonctionne en marge de tout système existant.

Les références à Samia Gamal sont primordiales, car tout au long de sa carrière, elle a 
incarné une grande source d'érotisme. En comparant Bintou à cette légendaire danseuse du ventre, Koffi Kwahulé subvertit la figure sacrificielle chrétienne. En plaçant son héroïne dans un contexte inattendu, soit celui de la mythologie chrétienne, il change de ce fait les rapports existants et remet en question les tabous d'ordre sexuel. Rappelons que le corps est souvent dévalorisé et que la chair est interdite dans la mythologie chrétienne. Ils sont jugés inférieurs à l'esprit. À ce sujet, ceci nous renvoie à saint Augustin ${ }^{35}$, dont les écrits ont grandement influencé le christianisme, qui est l'une des principales sources de la doctrine du péché originel, de la notion de l'infériorité de la chair par rapport à l'esprit, et en ce qui a trait à la culpabilité associée aux relations sexuelles. Pour saint Augustin, la fréquentation des femmes en vue du plaisir ou de la paternıté constituait un obstacle pour l'homme. Le péché résidait non pas dans l'acte sexuel, mais plutôt dans la motivation charnelle et égoïste qu'il sous-tendait.

Dans le second tableau de la pièce intitulé Jazz, Kelkhal et Blackout racontent leur désir pour Bintou, en parallèle aux événements associés à la danse du ventre et au meurtre d'un homme. En revanche, tout le discours de Manu, bien que l'on sache qu'il ait choisi Bintou au lieu du crack et qu'il soit devenu son petit ami, ne comporte aucune allusion à l'aspect sexuel de leur relation. L'ensemble de ses propos concerne plutôt la manière dont il pourrait épater Bintou, en accomplissant un geste encore plus grand que celui posé par Blackout. Manu affirme qu'il n'est pas jaloux de Blackout, qu'il considère plutôt comme son frère. En ce qui concerne le personnage de Manu, nous retrouvons en lui les deux pulsions les plus importantes de la vie humaine selon Freud, soit la pulsion de vie, par l'intermédiaire de l'attraction sexuelle, et la pulsion de mort. Lorsqu'elles sont réunies, elles produisent les actes les plus violents dont un des fondements est d'assurer la survie de l'individu.

Bintou est une figure messianique et mythique, à la fois africaine, chrétienne et moyen-

35 Dans l'artıcle «L'orıgıne gnostıque de la visıon négatıve de la sexualıté chez saınt Augustın», Françoıs Doyon explıque qu'après avoır mené une vıe de relatıve débauche, saint Augustın a finı par être dégoûté de la sexualıté et a prôné une abstınence sexuelle totale. 
orientale. C'est en la voyant danser que Kelkhal a compris qu'il n'entrait pas dans une bande mais plutôt en religion, une religion nouvelle d'où émerge du même coup une nouvelle mythologie culturelle, née de la fusion entre l'Afrique et la chrétienté, des territoires où les différences se retrouvent, s'opposent et se nourrissent tout à la fois. C'est en ces termes que Kelkhal parle de Bintou: «Instinctivement j'ai compris, en voyant sa beauté de fruit défendu, que je n'étais pas venu pour entrer dans un gang mais pour suivre, jusqu'aux falaises de l'absurde, une fillette de 13 ans» (15).

P'tit Jean la qualifie de sainte. Elle a en effet le pouvoir de rebaptiser les membres de son gang et de leur ordonner de la suivre, comme des disciples suivant leur maître. Il considère les trois garçons de la bande comme des rois mages fous-à l'image de ceux décrit dans l'Évangile selon Matthieu—qui sont venus d'Orient, de pays étrangers, en suivant son astre afin de rendre hommage à Jésus. S'il n'est pas question d'étoile dans la pièce, Manu parle d'une analogie avec le soleıl qui aveugle ceux qui regardent Bintou. «Elle est comme le soleil: plus on s'en approche, moins on la voit... The top, on peut rien contre. Je crois que j'ai dû trop m'en approcher» (15). Bintou peut également être considérée comme une pécheresse sans scrupule, telle Salomé dans l'Ancien Testament ou Marie-Madeleine dans le Nouveau Testament. Ici, la prostituée devient la sainte dans une nouvelle trinité féminine où la mère est à la fois enfant et diablesse, correspondant ainsi aux figures de la mythologie chrétienne. Elle représente également la figure matriarcale puissante de la société africaine et les jeunes chanteuses sexy des clips de hip-hopen jupes très courtes et qui secouent leur «booty» dans la figure des hommes. Elle réunit toutes les figures les plus puissantes de la femme Noire séductrice, transgressive, violente, indépendante et aimant le sexe. Bintou est aussi le serpent qui charme les hommes et Ève qui croque dans la pomme.

En tentant de joindre les rangs des Lycaons, P'tit Jean veut se rapprocher de Bintou. Elle lui demande de tuer un homme juste pour le plaisir, mais il n'en a pas la force. Bintou menace de 
le tuer à son tour et appuie même sur la gâchette, mais il n'y a pas de détonation. P'tit Jean s'écroule alors aux pieds de Bintou, en la couvrant de baisers. «Si vous saviez le bonheur qui m'a pénétré lorsque je lui ai baisé les pieds» (35) dit P'tit Jean à l'oncle Drissa. Le jeune homme compare ses baisers à une prière juste. «Ma mère croit que les prières, ce n'est que dans la bible. Pourtant, cette fille est tellement belle. La regarder, c'est prier» (36). Les rôles sont renversés lorsque Bintou devient une présence transcendante qui détient un pouvoir supérieur sur les autres. Même s'il agresse sa mère et se drogue, P'tit-Jean est incapable de tuer un homme sans raison, un constat qui attise l'impatience de Bintou qui, à son tour, cherche à l'éliminer symboliquement pour son manque de courage. Mais grâce à la force de sa présence et à son statut de femme «supérieure», Bintou transforme P'tit Jean. Et même si elle se comporte comme un homme pour diriger sa bande, Bintou demeure également très féminine et très puissante sexuellement.

P'tit Jean représente également Judas Iscariote, celui des douze apôtres qui a trahi Jésus. C'est en effet lui qui, en échange d'un peu d'argent, a facilité l'arrestation de Jésus par les grands prêtres. Par la suite, Jésus a été amené devant Ponce Pilate pour y être jugé. Dans Bintou, P'tit Jean est celui qui, en l'obligeant à revenir à la maison, livre la jeune fille à son oncle et à sa famille. En échange de Bintou, il obtient de la drogue et de l'argent.

Mais si P'tit Jean est le traître, il a d'abord été le disciple. Nous voyons comment, dans la lecture moderne de cette mythologie, il est celui qui est transformé par le pouvoir de Bintou, tout comme le sont les trois autres disciples impliqués dans la création de cette communauté particulière gravitant autour de la jeune fille. Pourtant, P'tit Jean, le faible adore Bintou, même s'il contribue à sa perte. P'tit Jean est transformé par sa présence mais, en même temps, ne peut s'empêcher de la trahir. Cela Bintou le devine, tout comme Jésus le savait au sujet de Judas.

P'tit Jean considère Bintou comme une sainte, car son amour est tellement grand qu'il peut sauver le monde. Cette image de grâce renvoie au Christ qui a donné sa vie pour sauver 
l'humanité. Il s'agit là de l'illustration qui donne tout son sens au rite expiatoire. Même si elle n'a que treize ans, Bintou sait qu'elle va mourir avant d'avoir atteint ses dix-huit ans. À l'image de Jésus qui, lui aussi, savait sa fin proche. Avant de mourir, il dit que «Tout est achevé» (Jn 18.30). De son côté, Moussoba, lors de la cérémonie d'excision, veut mettre fin au monde de l'Inachevé.

Le monde de Bintou est à son image: à 13 ans, elle n'est plus une enfant mais pas encore une adulte. Dans un contexte anthropologique, elle évolue dans un espace liminal où les lois de la société ne sont pas encore en vigueur. Bintou dirige cet «entre deux mondes» où elle reconstitue sa propre vision des choses de l'univers qu'elle dirige seule, à la tête de sa bande de garçons.

\subsubsection{L'excision: de la tradition au rôle de chef de bande}

La question de l'excision est au cœur de Bintou et de la mythologie africaine selon Koffi Kwahulé. Dans un sens, on pourrait penser que la pièce dénonce l'excision traditionnelle des petites filles dans certaines communautés africaines. Mais la démonstration du dramaturge est beaucoup plus complexe que cette seule thématique. Le renversement des rôles est devenu tel que l'ablation des organes génitaux devient le seul geste susceptible d'assurer le retour aux identités traditionnelles. Une femme qui n'est pas excisée est perçue comme impure et adultère, car on considère qu'elle n'est pas en mesure de contrôler ses pulsions sexuelles. De plus, si elle n'est pas excisée, elle risque d'empoisonner l'homme pendant un rapport sexuel ou l'enfant au moment de sa naissance. Selon certaines croyances religieuses, culturelles ou sociales, au sein des communautés qui pratiquent l'excision, le clitoris est un organe qui doit être coupé pour permettre à la fillette de devenir une femme.

Dans son livre L'excision, Françoise Couchard souligne que dans «les cultures africaines animistes ou islamisées, l'excision entrait dans un rituel puisqu'elle se pratiquait avant la puberté pour marquer le passage de l'enfance à la classe des femmes» (51). Elle note également que les raisons évoquées en faveur de l'excision sont multiples, telles que la prescription religieuse, les 
arguments moraux, esthétiques et prophylactiques, ainsi que des questions liées à la propreté, aux odeurs et à une certaine décence des organes génitaux féminins, à la préservation de la virginité ainsi qu'à la jouissance et au plaisir féminin.

Françoise Couchard rappelle également l'idée associée au renoncement à l'androgynie et à la bisexualité. L'excision préserverait en effet la fertilité. Pour qu'une femme devienne mère, elle doit renoncer à l'idée de gémellité, tel que l'explique Martine Lefeuvre-Déotte dans son livre L'Excision en procès : un différend cuturel?, où chaque être humain, qu'il soit homme ou femme, porte dans son corps et dans sa tête des attributs mâles et femelles. La femme doit donc «renoncer à ce qui rappelle en elle des vestiges ou des traces de l'autre sexe. C'est le cas du clitoris considéré comme un petit pénis avorté, atrophié, dressé comme une petite épée» (Couchard 61). Lors de l'accouchement, le clitoris pourrait nuire au passage de l'enfant et même le blesser.

La présence de l'exciseuse dans Bintou renvoie donc à la tradition. En effet, Moussoba explique à la mère que sa fille est possédée et qu'il faut, par conséquent, l'exciser sans tarder. «Wanzo, le démon de la luxure, a traversé les mers pour venir posséder le dard de ta fille» (31). Dans son ouvrage, Martine Lefeuvre-Déotte explique qu'un enfant doit être débarrassé de son wanzo «par la circoncision/excision» (116), où chez les Bambara notamment, «Purifier, c'est couper, trancher, blesser, corriger par le fer» (116). Ainsi, l'enfant, en passant à l'âge adulte, pourra devenir un être stable, puis se marier et procréer.

Le rôle de Moussoba l'exciseuse est de mettre fin au chaos, à la dépravation, d'imposer la clarté au corps de la fillette et dans la famille, afin que les identités puissent de nouveau aspirer à la stabilité et imposer l'autorité de la famille traditionnelle africaine sur la jeune fille. Cette clarté doit se faire par le rétablissement des frontières transgressées par Bintou, transgressions qui, en reniant l'autorité paternelle, ont entraîné une déstabilisation. Moussoba pourra ainsi rétablir les rôles stables de la tradition africaine et réinstaurer l'autorité en redonnant le pouvoir à la famille. La mère explique la honte du père sans emploi qui reste enfermé depuis des mois. Elle ajoute que 
sa famille est montrée du doigt, car elle est considérée comme le maillon faible de la communauté.

De plus, les transgressions de Bintou ne peuvent être admises dans cette communauté. Moussoba ne peut pas comprendre la raison pour laquelle l'adolescente vit avec un Blanc, car cette décision va à l'encontre des agissements autorisés par une certaine tradition et qu'elle brouille davantage les frontières de ce qu'il est permis ou non de faire. Toutes les transgressions de Bintou représentent la confusion sexuelle que l'excision est censée extirper:

Il faut de la clarté. Bintou traverse actuellement l'empire de l'incertitude, de l'équivoque et de l'ambiguité ; ce monde de l'Inachevé où une femme est aussi un homme et un homme une femme. Est-ce un hasard si, pourtant si jeune, Bintou tient tête aux hommes, à commencer par son propre père? Bintou ignore l'autorité masculine... Il faut de la clarté. Autrement, elle vivra sans époux, les hommes quı s'accoupleront avec elle seront tôt ou tard piqués par son dard et mourront. Si la stérilité ne se referme pas sur elle, le nouveau-né, lors du grand passage, sera également tué par le dard. Il faut de la clarté. Et mon couteau tranchera la confusion qui célèbre l'Inachevé. (33-34)

La rencontre entre les consciences identitaires africaine et européenne entraîne des conséquences néfastes: un insurmontable fossé s'est creusé entre la jeune fille et sa famille. Bintou est le produit de cette rencontre qui devient conflictuelle. Sans ambages, Koffi Kwahulé affirme ainsi qu'il n'est pas possible de vivre en Europe comme en Afrique, et que les habitants des anciennes colonies françaises ne peuvent pas vivre en France selon les traditions africaines.

La bagarre dans le bar de Nenesse constitue le point culminant du discours de l'auteur quant à cette remise en question des identités entre la tradition et la modernité. Ce lieu constitue le carrefour de trois mondes: l'Afrique, l'Orient et l'Europe. C'est pour cette raison que Bintou l'Africaine exécute la danse du ventre orientale et fréquente un Français, devenant ainsi le point 
de rencontre de ces trois mondes. Dans le bar, le juke-box joue une chanson française des années 1930. Lorsque Bintou tente de démontrer son savoir-faire à Nenesse, Manu met en marche un magnétophone qui diffuse une musique orientale. Puis arrivent P'tit Jean et ses Pitbulls dont le magnétophone crache un rap. Bien que le rap soit associé à la musique Noire américaine, il est également très populaire parmi les artistes Français d'origine africaine et parmi la jeunesse en général. S'ensuit une guerre de volume sonore entre la musique orientale et le rap qui a pour effet de noyer la chanson traditionnelle française. Les deux magnétophones sont ensuite éteints, laissant de nouveau la place à la chanson française. La bagarre qui éclate alors ramène, dans une nouvelle explosion de décibels, les musiques orientale et le rap. Quand, à la suite de cette rixe, une poursuite s'amorce à l'extérieur, seule la musique française subsiste alors que «le rap et la musique orientale meurent» (41). Koffi Kwahulé laisse entendre ici que, peu importe les rencontres et le mélange des genres, la chanson française aura toujours, symboliquement, le dessus sur les autres cultures.

Koffi Kwahulé signifie ainsi que les immigrants, qui se heurtent à des lois et à des politiques entrant en conflit avec leurs traditions, doivent s'adapter à ces nouvelles réalités. Sinon, ils en subiront de néfastes conséquences. Selon l'auteur, ils doivent s'intégrer à cette nouvelle société, au risque de ne pas pouvoir s'y développer en harmonie. Nous pouvons y discerner une critique des codes sociaux et culturels de l'Afrique qui prône la clarté des règles. Cette clarté se définit par le respect des traditions et de l'ordre établi. Par sa conduite, Bintou enfreint les codes et transgresse les lois de cette culture ancestrale qui, jusque là, n'avait jamais été remise en question. C'est pourquoi, dans la logique de Bintou, il n'est pas nécessaire de reconnaître l'autorité du père, ni de la mère, de l'oncle Drissa, de la tante Rokia, de l'école ou du reste de la société. La liberté sexuelle dont la jeune fille fait preuve découle de la même volonté de résistance. Selon elle, ces lois ne sont plus claires. De par sa fonction, Moussoba incarne l'autorité qui encadre la tradition et le rite de l'excision. Selon ses paroles, le monde de l'Inachevé 
est celui de l'Occident et du chaos. Et comme les rôles ne sont plus clairs pour Bintou, Moussoba doit les imposer par le couteau, car il n'y a pas de place pour l'ambiguïté ni pour la résistance. Afin de ne plus être rejetée par cette société construite sur des pratiques et des valeurs ancestrales, Bintou doit se plier au rite traditionnel des jeunes filles. Et seule l'excision peut redonner à Bintou, à son père et à sa famille leur place dans cette société. En chassant le démon de la luxure, la famille croit pouvoir retrouver cet équilibre si important aux yeux de la communauté.

Dans ce dernier tableau, tout juste après le geste de l'exciseuse, Bintou imagine qu'elle se marie. Ses Lycaons apparaissent à ses côtés. «Kelkhal est habillé en prince arabe, Blackout en prince africain et Manu ... en prince européen de la Renaissance» (45).Ces images renvoient aux représentations idéalisées que Bintou se fait de ses amis. Nous voyons comment Koffi Kwahulé réécrit les mythes de la nouvelle modernité urbaine. Il s'agit en fait du nouveau mythe multiculturel, constitué par des êtres d'un âge liminal qui évoluent entre les lois. Les Lycaons sont transformés en personnages traditionnels tirés, en quelque sorte, de l'imaginaire d'une fillette. Elle les voit à travers les yeux d'une enfant, malgré qu'elle ait été forcée de grandir prématurément. Cet échange entre l'oncle et Bintou démontre justement que, malgré les apparences, elle n'est pas encore une femme.

L'ONCLE. Quand as-tu cessé d'être une enfant, Bintou?

BINTOU. Qui t'as dit que j'avais cessé de l'être? (9)

Bintou raconte le nouveau mythe dont il est question et qui devient la fable de cette jeune fille de treize ans. Les lois des adultes l'ont poussée à la révolte. Dans cette enfance attaquée de toutes parts, cette scène se déroule alors qu'elle est entre la vie et la mort, suspendue entre le rêve et la réalité. Ce conte de fée représente donc cette nouvelle mythologie urbaine et moderne, le récit fondateur de ce «nouveau-monde» qui regroupe de nouvelles réalités pour les cités et les villes françaises qui ne sont plus des lieux exclusivement Blancs. Cet espace urbain est désormais 
un amalgame des différents groupes qui composent ce pays européen dans lequel Africains et Maghrébins côtoient les habitants dont les familles sont sur le territoire européen depuis plusieurs générations. Ceci, dans une véritable mixité, une nouvelle mythologie française au sein de cette génération qui est née de la révolte symbolisée par celle de Bintou. Cette société englobe toutes les origines-qu'elles soient française, africaine ou maghrébine - et tous ceux qui en font partie sont sur un pied d'égalité.

La cérémonie d'excision clôt le rituel et Bintou meurt parce qu'elle «n'a pas été courageuse» (46), selon Moussoba. Cette dernière rejette le blâme de la mort sur la fillette, seule responsable de ses choix et de son destin. Pour sa part, Françoise Couchard mentionne également cette question associée à une excision qui tourne mal. Encore aujourd'hui dit-elle, peu de chercheurs ou d'observateurs s'intéressent aux complications qui découlent de cette opération. Trop souvent, c'est la fillette plutôt que l'exciseuse qui est blâmée puisque cette dernière est très expérimentée. Françoise Couchard écrit que si «l'intervention se passe mal, c'est parce que la fillette a trop bougé ou parce qu'il y a eu une « mauvaise contention » de cette dernière durant l'opération» (68).

Dans la pièce, Moussoba, qui incarne la tradition, ne remet nullement en question ces pratiques. Il lui faudrait alors revoir son propre rôle au sein de cette société africaine qui n'admet pas d'autre vérité que la sienne. «Vous savez que ce que nous allons faire est sacrilège pour ce pays? Ce pays qui voue un culte à l'hérésie nous traite, nous les guérisseuses d'âme, comme de vulgaires assassins...» (33). Ainsi, Moussoba devait chasser le démon de la luxure et ramener l'ordre dans le corps de Bintou. Elle devait aussi lui permettre de renouer avec sa véritable famille, tout en éliminant la bande de Lycaons qu'elle lui avait substituée.

Ce rite devait également marquer le passage, pour Bintou, de l'enfance à l'âge adulte. Dans Je vois Satan tomber comme l'éclair, René Girard aborde également cette question ainsi que celle associée à la mort d'un des participants. 
Dans une première phase, qui est celle de «la crise», les postulants mouraient, en quelque sorte, à leur enfance et, dans la seconde phase, ils ressuscitaient, capables désormais d'occuper la place qui leur revenait dans le monde des adultes. Dans certaines communautés, il arrivait, de temps à autre, qu'un des postulants ne ressuscite pas, qu'il ne sorte pas vivant de l'épreuve rituelle et c'était de bon augure pour tous les autres postulants. On voyait dans cette mort un renforcement providentiel de la dimension sacrificielle du processus initiatique. (145-146). Dans la pièce, Moussoba ne remet pas en question cette pratique, rejetant même le blâme sur Bintou qui, d'après elle, a manqué de courage. Sa mort n'agit pas comme un élément de dissuasion puisqu'elle compte encore pratiquer trois autres opérations avant le lever du jour.

Bintou devient la victime symbolique d'un rituel de sacrifice. Elle représente donc une victime expiatoire de la douleur de tous les immigrants qui sont incapables de s'intégrer. Par le sacrifice, la famille et la société africaine retrouvent la clarté de la tradition et de son identité culturelle. Nous pouvons même avancer que la mort de Bintou peut être considérée comme un nouveau meurtre fondateur dans sa révolte contre ce monde de traditions qu'elle abhorre, mais dans lequel son entourage familial veut à tout prix la réintégrer contre son gré.

D'après le mythe expiatoire, Bintou incarne une victime qui périt selon les rites fondateurs d'une société traditionnelle. Le dramaturge démontre qu'un mythe fondateur prend alors naissance dans cet espace ainsi créé, bien qu'il soit à peine perceptible: celui d'une nouvelle mythologie urbaine d'une société de l'immigration. Il représente d'autres relations de pouvoir avec Bintou qui est adulée, adorée et à laquelle des hommes obéissent, bien qu'elle soit plus jeune qu'eux. Elle devient une sainte dont la mort engendrera symboliquement une nouvelle société, comme le décrit René Girard. Cette victime expiatoire sacrifiée permet l'émergence d'une société qui, à l'instar de tout récit dans lequel un meurtre fondateur est à l'origine d'un mythe, explique l'apparition d'un nouvel ordre du monde. «Les sacrifices ne sont rien d'autre pour commencer que 
la résolution spontanée, par la violence unanime, de toutes les crises qui se présentent inopinément dans l'existence collective») (Satan 146-147).

La mort de Bintou signifie l'arrivée d'une société de fusion des traditions, annoncée par l'entremise d'un texte fondateur qui entraîne la construction d'une héroïne mythique. Cette nouvelle société française pourra dorénavant être définie par tous les gens qui la composent. Mais avant d'y parvenir, Koffi Kwahulé démontre l'échec criant de l'immigration en France où, apparemment, aucune réconciliation n'est possible entre la tradition et la modernité. La mort de Bintou ne permet pas de croire que la pratique de l'excision cessera. Selon Girard, la mort renforce plutôt les croyances, érigeant un mur encore plus important entre ces deux mondes. Par la suite, la lutte musicale dans le bar de Nenesse rappelle la domination du pouvoir colonial. La musique française des années trente est la seule qui ait été en mesure de durer, alors que les mélodies orientales et le rap sont réduits au sılence.

En fait, Bıntou rêve d'une société plus juste où Maghrébins, Africains et Français seront conviés à vivre au même diapason, tout en respectant leurs différences mutuelles. À la toute fin, elle n'appelle plus ses trois amıs par leur surnom mais bien par leur véritable prénom. Okoumé, Kader et Emmanuel peuvent alors reprendre leur identité dans cette société où, enfin, ils ont une place. Le sacrifice de Bintou semble illustrer l'échec de la vision traditionnelle de la culture dans ce contexte de l'immigration. Elle se sacrifie pour que les jeunes puissent renaître dans un monde plus moderne et libéré de la tradition. Affranchis par la mort de Bintou, ses Lycaons peuvent recommencer à vivre par et pour eux-mêmes. C'est par la mort de cette victime expiatoire que Bintou pourra peut-être «sauver» le monde et ainsi, faire vivre ce nouvel ordre dont le sort dépendra désormais des jeunes générations. Comme Dieu qui a pardonné aux hommes de l'avoir renié et abandonné, Bintou pardonne à P'tit Jean de l'avoir livrée à sa famille. Elle le serre même contre elle après avoir été excisée. Par cette étreinte, Koffi Kwahulé signifie peut-être que cette société renouvelée ne pourra naître que si le pardon fait partie du processus de réconciliation. 
Dans notre analyse, au chapitre suivant, nous démontrerons comment le dramaturge s'est distancié des questions directement liées à la couleur de la peau, pour s'intéresser plutôt à des personnages perçus comme des étrangers par la société dans laquelle ils évoluent. Nous verrons alors que le paradigme inclut désormais toutes les figures de l'exclusion en France, qu'il s'agisse d'immigrants, d'étrangers, de Noirs ou de tous ceux qui sont marginalisés. Nous verrons comment ces personnages se heurtent à cette société française qui ne les reconnaît pas et qui les contraint à vivre dans la marge.

\subsection{Ave Maria et le meurtre fondateur}

La pièce $A v e$ Maria $^{36}$ renvoie au meurtre fondateur, celui commis par Caïn qui tua son frère Abel par jalousie, et dont il est question dans la Genèse. Ave Maria renvoie également à certains éléments de la messe catholique, dont Koffi Kwahulé semble s'inspirer pour adapter à la scène le premier meurtre de la Bible. La pièce est constituée de quatre tableaux respectivement intitulés Nativité, Messe basse, Prière et Confessions. Elle contient de nombreuses références au récit fondateur chrétien et nous verrons comment Koffi Kwahulé subvertit ces rites sous de multiples formes. Les sources du récit mythique inspiré de la Bible sont contenues dans l'Ancien et le Nouveau Testament. Dans l'Ancien Testament, il s'agit de la Genèse où il est question de la création, du jardin d'Éden, de l'histoire de Caïn et Abel et du meurtre de ce dernier par Caïn. Dans le Nouveau Testament, il s'agit des récits concernant la vie de Jésus, tels qu'ils ont été rapportés par les Évangiles de Matthieu, Marc, Luc et Jean, en insistant particulièrement sur les personnages féminins. Dans Ave Maria, Koffi Kwahulé amalgame et fusionne ces différents récits.

36 La pièce Ave Marıa, quı fatt partıe du recueıl Regards-9, Éd Lansman, 2008, comporte actuellement tro1s versıons. Dans le cadre de cette thèse, nous avons choısı de nous en tenır à la premıère de ces versıons, une commande du Théâtre Nıveau Parkıng et du Théâtre de la Bordée de Québec, à l'occasıon du $400^{\mathrm{e}}$ annıversaire de la ville de Québec, en 2008 La seconde versıon s'intıtule Madeleme Yebedhova (Ave Marıa \#2) et la trossième, Goutte d'or (Ave Marla \#3) 
L'Ave Maria est le nom latin de la prière Je vous salue Marie qui, chez les catholiques, est dédiée à la Vierge Marie. Pour les catholiques notamment, Marie est aussi la mère de Jésus de Nazareth. Cette prière s'adresse à tous ceux qui souffrent et qui sont seuls, puisqu'elle suggère qu'il y a toujours quelqu'un qui les écoute. En musique, il existe de multiples œuvres intitulées Ave Maria, dont parmi les plus connues figurent celles composées par Schubert, Verdi et Gounod (qui, pour sa part, s'est inspiré d'un prélude de Bach).

La pièce s'organise autour de trois personnages avec, d'une part, le couple formé par Yebed, un étranger, et Madeleine, née dans ce pays qui n'est pas nommé. Il est aussi question d'une autre figure qui arrive de l'extérieur. De passage chez le couple, Dhova, le frère de Yebed, tente de fuir momentanément la guerre qui déchire son pays. À l'origine, Dhova ne devait résider que quelques semaines chez Yebed et Madeleine, ma1s le voilà qui empiète de plus en plus sur leur intimité. Madeleine dit trouver cette situation insupportable et souhaite voir Dhova partir rapidement. Les prénoms de Dhova et Yebed évoquent des visiteurs venus du Moyen-Orient. Par contre, celui de Madeleine, bien qu'il soit très français, demeure des plus ambigus, du fait qu'il contienne de nombreuses significations.

En dégageant des histoires fondatrices de l'Ancien Testament et en les incorporant aux mythes fondateur de la pensée chrétienne — avec notamment l'évocation du jardın d'Éden et de la question du frère qui renvoie au mythe de Caïn et d'Abel-Koffi Kwahulé établit une synthèse. Dans la Bible, Ève enfante les frères Caïn et Abel. Dans ce mythe judéo-chrétien, où Caïn tue son frère par jalousie à la face du Seigneur, il est contraint à l'exil dans le pays de Nod, à l'est d'Éden. Il s'agit du premier meurtre perpétré dans la Bible. Le jardin d'Éden est la terre qui a accueilli Yebed pour lui donner une meilleure vie, bien loin de la guerre que Dhova connaît. Yebed prévient son frère qu'il ne doit pas s'opposer aux lois et aux règles de l'immigration, car «ils te donneront la chasse comme à un loup enragé» (26). Ce qui donne encore plus d'intérêt à cette situation est que Koffi Kwahulé l'insère dans le contexte très actuel de l'immigration, où les 
autorités pourraient effectivement chasser Dhova. À l'image de Dieu qui a chassé Caïn du paradis, le Dieu de la mythologie chrétienne devient l'État dans le contexte de l'immigration. Un État qui impose les règles, les droits de séjour et qui détermine la vie de l'immigrant, en lui accordant ou en lui refusant un quelconque statut dans le pays. Yebed devient justement le porteparole de cet État d'où Dhova pourrait, à tout moment, être chassé en tant qu'immigrant illégal. Dhova symbolise donc Caïn, celui qui contrevient aux lois et par qui le malheur arrive. Par conséquent, Yebed est tout à la fois le frère de sang de Caïn, soit la figure d'Abel et la voix du pouvoir de l'État.

Si le titre de la pièce est un hymne à l'intention de la Vierge Marie Mère de Dieu, il est également associé aux noms de Marie, la mère de Jésus, l'épouse de Joseph, ainsi qu'à Madeleine, aussi connue sous le nom de Marie-Madeleine. Dans la Bible, Madeleine évoque la pécheresse prostituée qui a lavé les pıeds du Christ avec ses larmes, puis les a essuyés avec ses cheveux. Koffi Kwahulé n'emploie pas le prénom de Marie pour son personnage féminın, mais dans le contexte de la mythologie catholique, il s'agit de Marie-Madeleine, personnage autour duquel il crée des associations troubles. Le nom de Marie / Madeleine entretient l'ambiguïté, car il remet en question le statut de pureté de la Vierge avec celui de Madeleine, la prostituée. Il oppose celui de l'Immaculée Conception —où Marie n'a pas été souillée par le péché originel et même celui de l'Annonciation où l'ange Gabriel annonce à Marie la conception virginale de Jésus par la vertu du Saint-Esprit-à celui de la prostituée.

Et c'est en assimilant les figures archétypales de la Vierge-Marie, de Marie la femme de Yebed et de Madeleine, la Putain, que le seul personnage féminin de la pièce devient subversif. En ce sens, la protagoniste correspond aux trois voix des figures archétypales de la femme inspirées par la mythologie catholique. Nous reconnaissons ces figures dans la pièce Les fées ont soif, de Denise Boucher. La dramaturge québécoise donne la parole aux personnages de La Statue, (la Vierge), à Marie (la mère et l'épouse), ainsi qu'à Madeleine (la prostituée) par des 
mises en abîme. Lise Gauvin, dans l'introduction de Les fées ont soif, traite justement du fait de rassembler ces trois images archétypales et «d'en faire une trinité opérante et parlante» (11). À la différence de Koffi Kwahulé, Denise Boucher a écrit une pièce féministe dans laquelle elle dénonce certains stéréotypes accolés à la femme, ainsi que les réactions de violences masculines qui en découlent.

Koffi Kwahulé renvoie lui aussi à une nouvelle trinité en jouant avec les trois images de la femme, la Vierge, la Mère et la Putain, qui sont les trois archétypes de la femme dans la religion catholique. Cependant, en réunissant ces trois femmes dans un seul corps, Koffi Kwahulé confère la même valeur à ces trois symboles de la femme. C'est en fusionnant la Vierge, la Mère et la Putain dans un seul et même personnage, qu'il change la dynamique du récit en les mettant toutes sur un pied d'égalité. Le fait de les réunir toutes les trois dans un seul corps devient subversif car il s'agit d'une nouvelle trinité féminine, à l'image de Dieu le père, du Fils et du Saint-Esprit. Cette trinité s'incarne en un être charnel et sexuel, où l'Immaculée Conception, l'Incarnation du Fils de Dieu en Jésus-Christ au moment de l'Annonciation et la maternité divine perdent tout leur sens. La mère de Dieu devient ainsi une femme comme toutes les autres, perdant ainsi son identité divine.

Cette trinité subversive, qui rassemble trois nouvelles figures archétypales catholiques, contient également tout le paradigme de l'exclusion. La signification de la femme devient plus complexe puisque l'auteur la situe dans le paradigme de tous les exclus de la société européenne: les immigrants, les étrangers, les sans-papier et même les Roms dans l'actualité française.

La pièce est organisée selon quatre tableaux qui, de manière générale, retracent le mouvement des événements et l'évolution des relations entre le couple et le frère venu d'ailleurs.

\subsubsection{Nativitié et rencontre}

Le premier tableau, Nativité, rappelle, selon la Bible, la naissance de Jésus le 25 décembre 
à Bethléem, où Marie accoucha dans une étable et où l'enfant fut déposé dans la crèche. Dans la pièce, Dhova, qui est nouvellement arrivé chez son frère Yebed, raconte à sa belle-sœur Madeleine l'arrivée au monde de son mari, le cadet de la famille. En effet, Yebed, son jeune frère, a vu le jour dans un taxi, en pleine tempête de neige, dans le pays d'origine de leurs parents, un endroit où il ne neige jamais. Koffi Kwahulé assimile le personnage de Yebed à Jésus, car tous les deux sont nés à minuit, dans des lieux qui ne sont pas destinés à accueillir les nouveaux-nés. Le dramaturge dresse un parallèle entre la nativité biblique et cette nativité contemporaine du mari dans un contexte moderne, et qui s'est déroulé dans des conditions semblables.

Pourtant, s'il est d'abord question de la naissance de la Nativité et de la naissance physique de Yebed, l'auteur met en relief une autre naissance symbolique dans ce tableau de la Nativité. Dans un premier temps, elle concerne l'arrivée de Yebed dans ce pays, et, dans un second temps, le souhait de celui-ci de voir son frère Dhova naître à son tour dans cet ailleurs. En effet, au moment où Yebed écrit à son frère Dhova pour l'inviter à venir le visiter dans cette «autre Amérique» (25) qu'il ne manquera pas d'adorer, il constate que c'est enfin dans ce lieu qu'il a «fini par naître, vraiment naître» (25). On comprend que cette naissance de Yebeb est en fait une «renaissance» dans ce Nouveau-Monde. Yebed espère que son frère finira, lui aussi, par vivre cette seconde naissance. Cette renaissance symbolique est en fait celle d'un étranger qui recommence sa vie dans un autre pays. Koffi Kwahulé pose la question de la possibilité de cette «renaissance» ou de ce recommencement pour Dhova, sans toutefois y apporter de réponse.

En effet, Koffi Kwahulé soupçonne que cette renaissance sera problématique et trouble. Le dramaturge semble signifier que la naissance n'est pas l'arrivée au monde d'un nouveau-né pur, innocent et sauveur de l'humanité, à l'image de Jésus. La naissance est ici plutôt symbolisée par des immigrants venus d'un autre pays et qui s'établissent dans l'espoir d'y trouver une nouvelle vie et un avenir qui leur permettra de se transformer. Le mythème concernant la structure de la naissance demeure le même, mais le contexte de ce mythème change quelque peu d'orientation et 
donc de signification avec la question de l'immigration et du statut de Dhova auprès des autorités. Koffi Kwahulé transforme tous ces mythes, qui à l'origine étaient clairs, pour les rendre très ambigus, jouant ainsi avec les différentes composantes du mythe. Et c'est là que réside toute la subversion de cette pièce.

Ce premier tableau montre le malaise de Madeleine qui ne supporte plus la présence de Dhova chez elle. Persuadé que Dhova ne possède aucun vêtement de rechange, Yebed accepte spontanément de lui en prêter. Mais sa femme ne voit pas les choses du même œil. Elle évoque un rapprochement sexuel entre les vêtements et la peau des deux frères. «Le voir dans tes chemises, dans tes pantalons, dans tes chaussures... rentrer dans tout ce qui est à toi... ça me donne la nausée» (24). Malgré les récriminations de Yebed, sa femme répète son dégoût. «D'imaginer une autre peau se glisser dans ces habits contre lesquels tant de fois ma chair a... Comme s'il se glissait dans ta peau... En moi» (25). Elle veut savoir quand le frère quittera la maison. En parallèle, Dhova affirme que son frère est chanceux d'avoir une femme aussi belle que Madeleine. Il n'est donc pas indifférent à cette dernière qui, de son côté, ne peut plus le supporter.

Les rapports d'ambiguïté se situent à plusieurs niveaux. D'abord Dhova emprunte les vêtements de son frère, indıquant une tentative symbolique d'assumer l'identité de Yebeb. De son côté, Yebed se trompe sur son frère lorsqu'il croit que celui-ci ne possède rien. Dhova pourrait avoir d'autres vêtements neufs mais il tient à porter ceux de son frère. Yebed le protège, car il se sent coupable vis-à-vis de lui qui vit toujours dans leur pays en guerre, alors que lui-même vit dans le confort. De son côté, Dhova exerce un chantage émotif dans le but d'obtenir la pitié de son frère. Madeleine en vient ensuite à faire un rapprochement sexuel entre les vêtements et la peau des deux frères. Mais son rapprochement est inversé: il ne s'agit pas de nausée mais plutôt de désir. Elle est gênée face à son désir inavoué pour Dhova, désir qu'elle tente de faire passer pour du dégoût lorsqu'elle se confie à Yebed. À cela, s'ajoute la convoitise sans équivoque de 
Dhova pour Madeleine. Il éprouve lui aussi du désir pour sa belle-sœur. Koffi Kwahulé établit ici des rapprochements sensuels et érotiques, des dires et des sentiments qui sont impurs et qui contreviennent aux Dix Commandements, où il est clairement écrit que l'homme ne doit pas convoiter la femme de son prochain ni commettre l'adultère.

En fait, ce qui est subversif dans la pièce est, comme nous l'avons vu, l'ambiguitté même de l'identité des personnages. Cette ambiguïté se retrouve également dans la manière dont le dramaturge joue sur les relations familiales, c'est-à-dire en montrant comment la femme est attirée par son beau-frère, et comment ce dernier exploite cette situation pour tenter de s'attirer la pitié de son frère. Nous avons vu que cette ambiguïté est évidente par l'utilisation même du nom de Madeleine, qui possède de multiples identités réunies dans cette trinité féminine (Vierge, Mère et Putain). Peu à peu, ces différentes tendances refont surface dans les relations avec les deux frères: Yebed le marı semble être plus naîf, alors que Dhova a l'air de mieux savoir profiter de la situation. Madeleıne a également un comportement très ambigu face aux deux hommes: elle veut que son beau-frère quitte la maison mais, en même temps, elle est très attirée par lui. Peu à peu, le personnage glisse davantage vers le modèle de Prostituée et s'éloigne de celui de Vierge. Les frontières entre les personnages archétypaux se brouillent. En situant les personnages dans les espaces liminaux où les identités deviennent instables, Koffi Kwahulé remet en question les fondements mêmes du mythe biblique.

Nous voyons comment Koffi Kwahulé transforme ce mythe pour lui donner un nouveau sens qui, bien qu'il emploie un vocabulaire directement emprunté à la religion catholique, confère cependant une ambiguïté aux personnages, brouille les pistes entre les figures du Bien et du Mal et change les rôles qui leur avaient d'abord été attribués. Ces figures ne constituent pas une seule présence mais la somme d'identités multiples. En ce qui concerne Madeleine, elle est à la fois Marie la pure et Madeleine l'impure, qui représentent également la Vierge Marie, soit l'Ave Maria du titre de la pièce, et la prostituée de la Bible. 
Dhova, le frère récemment arrivé, semble aussi représenter Caïn. Si ce personnage n'est pas ambigu, le rapport qu'il établit avec la femme de son frère lui, le devient. Bien qu'il aime son frère, Dhova veut le trahir en lui volant sa femme. Il brouille lui aussi les pistes entre l'amour fraternel et la trahison ultime de son frère.

Quant à Yebed, il incarne à la fois Dieu—qui est ici représenté par l'autorité et le pouvoir de l'État relatif aux lois de l'immigration—le frère bienveillant et, ultimement, le mari qui est trahi par son frère. Yebed assume de nombreux rôles qui peuvent parfois sembler ambigus, car il détient le pouvoir symbolique de l'État. À la toute fin de la pièce, Yebed se transforme en JésusChrist, l'essence de l'Esprit chrétien, pardonnant à sa femme et à son frère qui le quittent pour partir vivre ensemble. Ces rapports troubles au sein même des personnages sont bien illustrés par les différents tableaux de la pièce.

\subsubsection{Messe basse}

Dans le second tableau, intitulé Messe basse, les trois personnages sont de nouveau réunis. La messe chez les catholiques est une célébration religieuse qui, lors de la Communion, constitue un rite sacrificiel où le pain et le vin rappellent symboliquement le sang et le corps du Christ. Il existe différents types de messes: la messe traditionnelle, la grand-messe et la messe basse. Dans ce dernier cas, il s'agit d'une messe dite à voix basse, sans musique ni chants et où tout est récité. Au sens figuré, il s'agit de dire quelque chose à voix basse afin de ne pas être entendu par ceux qui nous entourent.

Dans la pièce, la messe basse évoque également un jeu de mots ambigu qui peut être associé au bas du corps, à la chair et à la sexualité. Elle sous-entend une célébration qui refuse le mystère de la messe catholique, ${ }^{37}$ mettant plutôt en valeur des rapports du corps beaucoup plus charnels. Il s'agit d'une subversion de la messe chrétienne, qui représente la rencontre entre deux

\footnotetext{
37. Par l'utılısatıon du pain et du vın, la messe catholıque commémore la dernıère Cène et le sacrıfice du Chrıst.
} 
mondes, celui plus inquiétant de Dhova par rapport à celui plus traditionnel de Madeleine.

Dans ce tableau, Koffi Kwahulé fait intervenir la messe basse à deux reprises, pour signifier la transformation des rapports et le rapprochement entre Madeleine et Dhova. Dans un premier temps, Dhova raconte à Yebed qu'il a été témoin d'une dispute entre un homme et une femme, dans une église qui a été transformée en bibliothèque, un lieu profane. Cette transformation d'un lieu de culte en lieu de savoir et de raison, permet justement de constater les transformations associées à la messe basse. La bibliothèque n'est plus seulement un lieu de spiritualité et de croyances, mais plutôt un endroit où, par l'émergence d'autres types de pensées, il devient possible de remettre en question l'existence de Dieu. Dhova est donc un intrus, un voyeur dans ce lieu et dans la scène qui a lieu au sein du couple. Il évoque cette scène telle l'Annonciation de l'ange Gabriel à Marie, qu'il sera lui-même appelé à jouer plus tard avec Madeleine. Koffi Kwahulé continue à changer les identités et les fonctions des personnages, allant jusqu'à transformer momentanément Dhova en ange Gabriel.

Dhova explique à Yebed: «Ils chuchotaient. Une scène étrange. Une séparation, je présume» (28). En effet, la femme essayait de repousser l'homme qui tentait de lui glisser sa main entre les jambes. «Aussitôt la femme a serré les cuisses pour arrêter la reptation de la main de l'homme» (28).

La reptation renvoie à l'action de ramper, comme le font les reptiles et, donc, les serpents qui représentent, dans l'iconographie gréco-chrétienne occidentale, la connaissance et le mal, mais également le sexe de l'homme. Cette connaissance est elle-même associée au savoir contenu dans les livres des bibliothèques. Il est donc question d'un des sept péchés capitaux, celui de la luxure qui renvoie à la tentation (dans l'Ancien Testament) et à l'assouvissement des plaisirs charnels. Koffi Kwahulé tisse des liens complexes entre les différents protagonistes en fusionnant plusieurs images concernant Adam et Ėve. Il est question du serpent, de la tentation, du plaisir, du mal, de l'expulsion du jardin d'Éden, de l'éveil de la conscience des plaisirs charnels et de la 
connaissance. Dans la bibliothèque, le serpent constitue l'intrus sexuel alors que les livres apportent le savoir. Tous ces éléments permettent de tisser d'autres liens avec la Bible.

La messe basse évoque donc le bas du corps qui représente la subversion du mystère catholique. Dans la bibliothèque, Dhova écoute la femme qui dit à l'homme: «Ne profite pas de ma faiblesse. C'est fini. Envers lui j'ai péché. Envers lui tu as péché. N'en rajoutons pas» (28). Il est aussi question de «l'âcre encens de l'apostasie qui te damne à moi» (28), l'apostasie étant aussi appelée la débaptisation dans le christianisme. Durant cette scène de la Messe basse, les amants chuchotent parce qu'ils sont dans une église transformée en bibliothèque, un lieu désinvesti de sa signification religieuse. Mais il faut également souligner le fait que le contenu de leur échange, bien qu'll soit indicible, attente à la pudeur et constitue un véritable sacrilège, tout particulièrement dans ce lieu symbolique, autrefois sacré. Koffi Kwahulé désacralise cette scène en lui donnant une signification profane.

Dans un second temps, Madeleine raconte aux deux frères qu'elle a été témoin d'une scène de rupture survenue dans les toilettes d'un centre commercial, à Sainte-Foy. Les toilettes publiques sont parfois associées aux rencontres sexuelles clandestines. Nous pouvons y voir une certaine ambiguïté sexuelle ou, encore, une sorte d'opposition perverse de lieu par rapport à la bibliothèque-église. À la bibliothèque, le couple s'était retrouvé dans l'absidiole, une sorte de petite chapelle, alors qu'au centre commercial, il se retrouve dans une cabine des toilettes pour femmes, un endroit qui est également chargé symboliquement. Cet épisode, où Madeleine raconte la scène, est en fait l'incarnation des fantasmes de Madeleine et de Dhova attisés par le désir mutuel, indicible et interdit de l'autre. Ces fantasmes conditionnent ensuite leur comportement, tout particulièrement lorsque la femme demande à son mari de la masser mais que son beau-frère s'empresse de la satisfaire en premier. Ces parallèles plongent de plus en plus Madeleine et Dhova dans la transgression, établissant ainsi les rapports avec la messe basse. Il s'agit d'une profonde remise en question de la doxa sur les rapports entre l'Église et les activités sexuelles, 
pratiques condamnées par saint Augustin dès le $I V^{\mathrm{e}}$ siècle de notre ère.

Par la suite, bien que Madeleine demande à Yebed de lui masser le dos c'est Dhova qui s'en charge. Il s'en suit entre les deux protagonistes le même échange que celui du couple entendu dans la bibliothèque, car les massages de Dhova se sont transformés en caresses. Il s'agit d'une transposition des événements du récit auxquels participent Madeleine ainsi que Dhova, l'étranger et l'intrus dans le couple. Les fantasmes évoqués dans la bibliothèque se concrétisent ici et se transforment en trahison pour Yebed. En effet, toute cette scène se déroule pendant que le mari s'est brièvement absenté et alors que le contact physique s'est intensifié entre sa femme et son frère.

En lutte contre ses remords, Madeleine lance ensuite le verre d'eau que son mari vient de lui apporter au visage de Dhova, lui annonçant qu'elle va tout avouer à Yebed. Mais Dhova lui rappelle les motıvations profondes qui justifient leur liaison. Il dit à Madeleine: «Au fond tu as dorloté mes sentiments pour jouer à cache-cache avec l'ennui d'un couple qui s'enfonçait dans le rance. Tu n'auras pas à le lui dire. Je ne t'imposerais pas cela. Je m'en vais. Je t'aime» (30). Cette odeur rance est celle qui émane des toilettes publiques et qui confère ce parfum d'ambiguïté générale à la scène. Et soudain, Madeleine redevenue momentanément la pure, retrouve son mari, témoin embarrassé de la scène. Elle lui raconte alors la fin de l'histoire survenue dans les toilettes, tout en lui demandant pardon.

Toute la scène constitue une ambiguïté explicite, car elle est construite sur la superposition de la scène observée par Dhova à la bibliothèque et de celle jouée par Dhova, Madeleine et Yebed, où ce dernier tient le rôle du voyeur. Il s'agit d'un passage qui intègre des moments de théâtre dans le théâtre, dans lequel tous jouent symboliquement des scènes interdites de séduction qui, implicitement, expriment leur désir à l'égard de cette relation illicite. Ce jeu théâtral leur permet de laisser déborder leurs sentiments et d'exprimer ce qu'ils ressentent vraiment, en tombant dans les bras l'un de l'autre sans trop s'avouer coupables. 
Dans cette messe basse, il y a une ambiguïté entre le ludique et le comique, entre le désir et la culpabilité, entre celui qui devient voyeur et celui qui a honte de regarder. Les moments de jeux sont encadrés par des renvois à la Messe basse, à la Prière et aux Confessions, qui constituent les différents tableaux de la pièce. Koffi Kwahulé établit une ambiance de transgression où le jeu n'est pas réel, laissant planer le doute, étant donné que le baiser est ambigu et que le mari le perçoit comme tel. Il s'agit même d'une subversion du théâtre, car il est difficile d'établir la manière dont cette mise en abîme fonctionne.

En plus de la transposition et de la désacralisation du récit biblique, il s'agit d'une reconstitution et d'une relecture d'un mythe fondateur. Par leur manière d'interpréter ce qui se passe dans le monde autour d'eux, chacun des personnages semble projeter des images qui correspondent à leur conscience profonde. L'allusion au serpent de Dhova renvoie au sexe de l'homme et particulièrement à celui de l'étranger. Cette image du sexe concrétıse la manière de percevoir l'étranger, celui qui provoque la peur, qui a le pouvoir d'attaquer et de violer et qui est synonyme de lubricité. Il s'apparente à l'animal, à son instinct et à son côté sauvage qui, lui, s'oppose à l'homme et à sa raison. L'inconscient de Madeleine, la putain, interprète dans les toilettes la scène qui correspond à son propre vécu. Les toilettes publiques résument à elles seules tous les maux de la terre: prostitution, drogue, rapports cachés de misère homosexuelle ${ }^{38}$ et présence de sans-abri. Il s'agit d'un lieu où tout ce qui est dégradant peut de dérouler. Tout y est dégoût et humiliation, puisque tous les déchets du corps s'y mélangent: vomissure, matières fécales, urine, sang, pourriture, odeurs nauséabondes. C'est la raison pour laquelle Madeleine et Dhova se rencontrent et se retrouvent dans ce monde de saleté et de souillure. Ils appartiennent au même paradigme puisqu'ils s'y reconnaissent tous les deux, lui en tant qu'étranger et elle, en tant que prostituée.

38 À ce sujet, nous pouvons tracer un parallèle avec le film L'homme blessé (1983) de Patrice Chéreau où, dans les couloırs d'une gare de province, un jeune homme de 18 ans falt la rencontre d'un voyou plus âgé qui le pousse à se prostıtuer Dans la puanteur, les tollettes sont un lieu où la sexualité et la violence se côtorent 
Cette Messe basse est donc une manifestation des désirs de Madeleine et Dhova, à partir d'un jeu de mise en abîme, du théâtre dans le théâtre. Cette relation reste malgré tout indicible, même si leurs désirs fantasmés semblent se concrétiser. Tous deux peuvent laisser déborder leurs sentiments et exprimer enfin ce qu'ils ressentent l'un pour l'autre. Quant à Yebed, il demeure le témoin silencieux de ce jeu qui n'en est pas tout à fait un, mais dont il est difficile de cerner les véritables contours.

\subsubsection{Prière}

Le troisième tableau, intitulé Prière est très court. Il renvoie à l'Ave Maria de Verdi, alors que le DVD offert à Dhova par Yebed joue cette musique à la télévision. Cette prière est en fait une séance de masturbation pour Dhova. Par la suite, les didascalies indiquent que Madeleine et Yebed étaient apparemment là depuis le début, alors que Yebed enlace sa femme pour la protéger de ce qu'elle pourrait voir. Pour Dhova, cette prière à la Vierge est une tentative de se rapprocher de son frère avec qui il partage tout, étant donné qu'il ne s'agit que de corps interchangeables. «Ce qui est à toi, ce quı est à moi... Les corps traversaient les mêmes habits... les mêmes...» (27), dit Dhova dans le tableau précédent. Et Yebed de lui répondre que Madeleine «est née ici» (27), sous-entendant qu'elle ne peut comprendre tout ce qui les unit. Cet adultère entre la femme et le beau-frère ne semble pas provoquer d'émotions chez Yebed, comme si cette situation était normale, voire attendue ou souhaitée, même si, dans les faits, il perturbe l'ordre établi et la vie du couple. Yebed ne répudie pas sa femme et semble vouloir lui pardonner cette liaison. Même Madeleine se considère comme une victime face à sa propre infidélité. Elle semble outrée, dégoûtée par le geste de Dhova. «Pourquoi faut-il que ce soit sur moi que s'abattent ces choses-là, dans une ville presque familiale?» (31). Mais dans les faits, elle refoule ses propres sentiments d'attraction pour Dhova, passant de la figure de la Prostituée à celle de la Vierge.

Yebed assure sa femme que tout sera fini le lendemain. Ainsi, après la Messe basse et la 
Prière, ces bouleversements issus du bas monde prendront fin avec le départ de Caïn / Dhova, alors que l'ordre des choses, dans le couple, reprendra vraisemblablement son cours d'avant l'arrivée du frère sous leur toit.

Comme nous l'avons vu, l'Ave Maria est une prière dédiée aux hommes et aux femmes qui souffrent, pour leur rappeler qu'ils ne sont pas seuls. Il s'agit d'un hymne qui est souvent associé aux célébrations de la nativité de Jésus. Dans ce tableau, la masturbation de Dhova est en fait l'incarnation de sa solitude, en tant qu'homme mais aussi en tant qu'étranger.

Koffi Kwahulé pose peut-être la question d'une nouvelle naissance pour chacun des personnages, étant donné que leur vie reprendra après avoir été bouleversée par la venue du frère. De plus, le tableau démontre la tension qui existe entre la dégradation associée à la messe basse et à la sexualité, par rapport à l'exaltation de l'opéra classique qui fait partie de la grande culture. Cette subversion rappelle l'œuvre du cınéaste Luis Buñuel qu1, dans ses films, ${ }^{39}$ s'attaque à la bourgeoisie, à l'Église et à l'hypocrisie sociale.

\subsubsection{Confessions}

Confessions, le dernier tableau, débute alors que Madeleine et Dhova rejouent la scène des amants qui se quittent à la bibliothèque et dans les toilettes publiques. À la télé, l'image montre l'église Saint-Roch, puis différentes églises de la ville défilent à l'écran. Les confessions dont il est question ne peuvent avoir lieu que devant ces églises, les portes des divers sacrements de l'Église catholique, dont celui du mariage. Yebed s'étonne de trouver Madeleine et Dhova ensemble, à la maison, alors qu'ils devaient être à l'aéroport pour le départ du frère. Madeleine annonce à Yebed qu'elle a dissuadé Dhova de partir et ce, même si ses papiers n'étant plus en règles, il devenait dès lors un hors-la-loi. Elle rend compte à son mari de la discussion qu'elle a

39 Notamment dans Le fantôme de la liberté et L'Ange exterminateur, ma1s également dans La vole lactée, un film qui traite de la relıgıon catholıque dans un "catalogue des hérésıes (l'eucharıstıe, la nature du mal, le Chrıst, la grâce et la liberté, Marie. )» (Tulard 2 1344) 
eue avec son beau-frère: «Il dit qu'il n'est jamais né. Il dit qu'il aimerait qu'on lui offre sa chance. Il dit que je suis son pays» (33). Koffi Kwahulé marque ici le retour à l'idée de la naissance concrétisée par l'arrivée de l'étranger dans le Nouveau-Monde. Mais il s'agit d'une renaissance avortée pour Dhova. Madeleine se joint à lui pour l'aider à naître de nouveau. La présence de la femme symbolise l'espoir d'un avenir meilleur, à l'image d'une mère qui se veut rassurante face à l'inconnu.

Yebed propose à sa femme de venir chercher ses effets personnels, mais elle lui réplique que c'est elle qui le quitte, pour «cacher ma nudité loin de la face de notre sacrement» (33) et parce qu'elle a trahi ses vœux de mariage. Le couple s'était juré fidélité lors de leur union devant l'Église. Dans la Genèse, il est écrit qu'Adam et Ève «étaient nus, l'homme et sa femme, sans se faire mutuellement honte» (Gn 1.25). C'est après avoir goûté au fruit défendu qu'ils ont découvert qu'ils étaient nus et qu'ils furent expulsés du jardin d'Éden. L'auteur établit un parallèle entre Ėve et Madeleine, la pécheresse qui n'a pas respecté ses vœux. Elle se doit de cacher sa nudité à son mari qui, pour sa part, aurait préféré ne jamais surprendre les amants. En ayant des relations sexuelles avec son beau-frère, Madeleine a ainsi goûté au fruit défendu. Cette scène fait référence à celle où Ève est chassée du paradis terrestre, une situation qui renvoie à la manière dont les immigrants illégaux, notamment les Africains en Europe et les Mexicains aux États-Unis, sont expulsés par les autorités qui les considèrent comme des criminels.

Les mythèmes inspirés du récit biblique relativement au paradis terrestre demeurent les mêmes, bien que Koffi Kwahulé change quelque peu la dynamique qui unit les protagonistes. Ainsi Yebed devient Adam qui, lui, demeure au paradis, alors qu'Ève et Caïn choisissent de partir volontairement à la recherche d'une nouvelle terre d'asile plutôt que d'en être chassés. Symboliquement, la mère part en compagnie de son fils, en laissant le mari derrière eux. Il s'agit alors d'une autre forme de subversion, car il est question d'inceste, l'un des grands tabous de la société moderne. Le dramaturge donne une nouvelle orientation au mythe où les rôles des 
personnages bibliques d'Adam, Ėve, Caïn et Abel sont réinterprétés au sein d'une fonction trouble.

Yebed affirme à sa femme: «Dis-lui qu'il a la bénédiction de son frère. Toi aussi tu as ma bénédiction» (33). La bénédiction est une courte prière ou un geste qui invoque la bienveillance divine. Mais Madeleine n'en veut pas. «Ne me bénis pas, pardonne-moi» (33), lui demande-t-elle au son de l'Ave Maria qui résonne de plus en plus. Alors que Yebed accorde sa bénédiction à sa femme et à son frère, Madeleine réclame plutôt son pardon, voire l'absolution de son mari qui, tel le Christ sur la croix, sacrifie son amour afin de sauver les pécheurs qu'ils sont devenus. Comme nous l'avons vu, Yebed joue à la fois le rôle d'Abel, du Christ et de la voix de l'État.

Ave Maria est donc une relecture contemporaine des récits bibliques dans laquelle Koffi Kwahulé brouille les pistes et réinvente les mythes chrétiens. Il se sert des récits bibliques et des éléments de la mythologie judéo-chrétienne pour mettre en valeur la nature même du rejet et de l'exclusıon, qui sont symbolısés par cet Autre, l'étranger, l'immigré venu d'ailleurs.

Cette nouvelle orientation émane d'une sexualité débridée et incontrôlée. Elle agit à un niveau fantasmatique refoulé qui découle de mises en abîme théâtrales à propos des rencontres indicibles dans la bibliothèque. Ces rendez-vous clandestins sont assimilés à la messe basse qui se déroule également dans les toilettes publiques, démontrant ainsi une transition entre la tête et la raison, vers le bas du corps. Il s'agit de gestes associés au corps et au bas du corps dans les toilettes publiques, de la destruction de l'identité familiale qui est elle-même basée sur les valeurs religieuses catholiques où la sexualité se limite à la procréation. En les transformant, il accorde une modernité à ces nombreux renvois sexuels qui occupent une grande importance du fait qu'ils sont basés sur la peur de l'Autre. Cette subversion s'alimente et se nourrit de la peur de la présence de l'immigrant, qui change radicalement la nature même de leur civilisation.

Koffi Kwahulé semble jouer sur cette notion de transformation qui peut se faire uniquement à partir de rencontres avec ceux qui viennent d'ailleurs. Il ne remet pas en question 
des rapports avec une autre religion mais souligne plutôt le fait que la présence de ces nouveaux venus perturbe l'ordre établi. Il l'explique à partir d'une déconstruction des concepts de base de cette opposition entre la pureté et l'impureté, entre la chair et le corps, et entre le Bien et le Mal. Il s'agit d'oppositions qui sont clairement ancrées dans l'idéologie de cette mythologie. Il exprime et critique même cette peur par un jeu de mises en abîme théâtrales, remettant en question les mythes qui constituent le fondement même de cette société.

Toutefois, cette subversion demeure profondément ironique, car cet ensemble consiste, malgré tout, en un jeu théâtral qui se déroule au sein de mises en abîme. L'acte de désacralisation est différé au théâtre. Par le fondement ludique de sa représentation, il devient ainsi un jeu ironique.

Cette ambiguïté pourrait même laisser entrevoir une certaine forme de caricature. Plusieurs tons sont possibles, allant de la comédie à la tragédie. Nous pouvons renvoỳer à l'importance de l'Ave Maria, une musique associée à la spiritualité qui, pourtant, est jouée pendant une scène à caractère sexuel. Tout au long de la pièce, Koffi Kwahulé met en place une certaine dialectique, un jeu d'oppositions, sans toutefois offrir une conclusion univoque.

De plus, la nature de cet Autre est fondée sur une vision «pervertie» de la sexualité dans laquelle cet étranger a le pouvoir d'obtenir les faveurs sexuelles des femmes, d'avoir des enfants et donc, de transformer cette société qui l'accueille. L'Église catholique n'admet pas la sexualité en dehors de l'amour conjugal. Pourtant Yebed est impuissant face au désir et à la passion des amants, après que Madeleine ait commis le sacrilège ultime envers lui, jouant tout à la fois, le rôle de Vierge et de Putain amoureuse.

Même si Yebed est irréprochable par rapport à Dhova, il n'est pas à l'abri de la destruction. Le mari n'est plus véritablement un frère, mais plutôt un membre appartenant de plein droit à cette société qui est ainsi mise en péril. Cette destruction est, comme nous l'avons vu, celle de la structure familiale issue des valeurs fondamentales de l'église catholique. C'est en cela que la 
subversion opère puisque Koffi Kwahulé déconstruit les bases de cette institution millénaire, transformant les récits bibliques pour en forger d'autres beaucoup plus subversifs.

Au tout début du dernier tableau, Madeleine raconte à Dhova sa naissance dans un cinéma. En effet, elle a vu le jour dans une salle de projection, le Bijou. Par la suite, l'endroit a, été transformé en cinéma «de mauvaises mœurs» (32). La transformation s'opère ici à deux niveaux. Premièrement, lors de l'accouchement, la mère n'a pas éprouvé de douleurs. Elle a même employé le mot «grâce» pour décrire l'événement. Les autres spectateurs lui sont venus en aide: une sage-femme qui a coupé le cordon ombilical et un homme qui a arraché un morceau du rideau qui protégeait l'écran pour l'y envelopper. Les gens ont été bons envers la mère et l'enfant. Nous pouvons voir un déroulement parfait pour accueillir le bébé, qui symbolise la pureté et l'innocence.

Par la suite, le Bijou a périclité et a été transformé en cinéma pornographique. Il s'agıt du second niveau de lecture, alors que des images sexuellement explicites sont présentées à une clientèle cachée dans l'obscurité, un endroit discret dans lequel peuvent se dérouler tous les gestes interdits possibles. La transformation du cinéma grand public en cinéma pornographique est à l'image de Madeleine qui, de son statut de nouveau-né touché par la grâce devient la figure emblématique de la Putain.

Il faut également souligner que le cinéma était situé non loin d'une ancienne synagogue qui, elle aussi, a changé de vocation pour devenir un théâtre. Il s'agit ici d'une transformation des institutions. Le lieu de culte sacré accueille désormais des fidèles qui assistent à une autre sorte de cérémonie, celle offerte par le théâtre. Comme l'Église catholique devenue bibliothèque, la synagogue juive est maintenant le lieu de rencontre des acteurs, sur cette scène qui symbolise l'érosion des valeurs traditionnelles, qui se traduisent par l'absence de fréquentation des églises au profit d'institutions culturelles, tout à la fois populaires et urbaines.

Il semble que la ville de Québec, aussi connue sous le nom de ville aux cent clochers, a 
profondément inspiré Koffi Kwahulé. Même si la ville n'est jamais nommée, plusieurs lieux sont indiqués. La ville et ses environs influencent grandement Dhova quand il parle de ses visites à son frère: «Parce qu'il n'y a pas que la mélancolie des églises. Ça te donne envie de croire en quelque chose, cette ville. Envie de devenir autre chose. Quelqu'un de bien... Juste quelqu'un de bien. Une envie de naître à nouveau...» (28). Nous pouvons également établir un rapprochement entre la Haute-Ville et la Basse-Ville de Québec. Ces éléments d'opposition figurent en effet dans la pièce, marquant le clivage géographique et social entre les riches dans la Haute-Ville et les pauvres dans la Basse-Ville. Quand Dhova arrive de l'étranger, il habite dans la Haute-Ville, alors que Madeleine et Yebed habitent tout en bas, «là où l'Amérique a goût d'Amérique» (26). Cette Basse-Ville donne un aperçu de ce que peut être l'Amérique, cet ailleurs porté par l'espoir d'une vie meilleure, malgré un environnement précaire et sans pouvoir. En situant d'abord Dhova dans la Haute-Ville - avant qu'il ne «descende» dans la maison du couple-Koffi Kwahulé représente encore une fois la crainte de l'étranger. Ainsi, Dhova pourrait s'emparer du pouvoir, le siège du pouvoir politique du Québec étant justement situé dans la Haute-Ville. En abusant du système qui a été mis en place pour aider les plus démunis de la société et les nouveaux arrıvants, l'étranger pourrait même bénéficier d'un pouvoir financier important ou d'une aide pécuniaire excessive.

Il pourrait aussi s'agir d'une division entre le bas et le haut du corps, entre le sexuel et la raison. L'épisode de la bibliothèque située sur le rue Saint-Jean, tel que raconté par Dhova, sa déroule dans la Haute-Ville, à la limite de la Basse-Ville. Il s'agit d'un lieu limitrophe délimité par la présence d'une falaise, un endroit un peu flou qui pourrait illustrer le glissement entre le savoir et l'érudition, vers le plaisir de la chair, tel que le plaisir de Madeleine et Yebed où les sens ont pris le dessus sur la raison.

La sonorité du prénom Yebed pourrait renvoyer à celui Yahvé. Selon l'Atlas des religions, Yahvé est le nom que les Hébreux donnent à Dieu. Il signifie «Celui qui est» et dont «on ne saurait le mettre en image et son nom même est imprononçable» (22). Par ailleurs, au temps des 
Hébreux, la loi du lévirat, qui visait au maintien des familles, était également associée au mariage. À la suite de la mort du mari, et si le couple n'avait pas eu de descendant mâle, elle obligeait un homme à épouser sa belle-sœur. Dhova et Madeleine forment ce nouveau couple, Yebed et sa femme n'ayant pas eu d'enfant. Mais ce mariage, qui est ici tout aussi symbolique que la mort de Yebed, n'est pas gage de bonheur. Les amants devront se rendre aux autorités pour tenter de régulariser la situation de Dhova ou fuir pour rester uni.

Dans le second tableau, Dhova avoue même qu'il a mal agi envers ses hôtes: «Madeleine et toi, vous êtes tellement généreux... J'ai trop abusé de tout. Tu sais, j'ai honte» (27). De plus, Dhova pourra être chassé du pays à cause d'irrégularités liées à son statut. Il est donc à la fois Caïn / Dhova où, dans le contexte de l'immigration, il est un sans-papier quı va à l'encontre des loi édictées par l'État.

Dans le dernier tableau, les amants rejouent la scène de la rupture «en riant» (32). Alors qu'ils s'amusent à se poursuivre dans la maison, ils sont interrompus par le mari qui rentre du travail et qui s'excuse même de ne pas avoir frappé avant d'entrer. Malgré le sérieux de la situation, nous y voyons tout de même une certaine approche vaudevillesque de la part de Koffi Kwahulé, car il transforme ce triangle amoureux en quelque chose de moins tragique qu'il n'y paraît.

Dans ce tableau, le dramaturge instaure une forme de distanciation en mettant en jeu des éléments appartenant au vaudeville, tels que le fait d'entrer sans avoir préalablement frappé, l'arrivée inattendue du mari cocu qui prend les amants sur le fait et la présence du triangle amoureux. Koffi Kwahulé fait appel à ces conventions théâtrales, même si son propos est plus complexe et significatif, allant jusqu'à mettre à mal la Bible. Il opère une rupture stylistique qui impose une distance avec le spectateur.

Selon Pavis, il y a rupture de jeu quand un comédien «change de registre, mêle les tons et casse l'unité de son personnage» (351). De plus, il définit les fonctions des ruptures comme étant: 
«Essentiellement moyen de distanciation, les ruptures sont la marque d'une esthétique du discontinu et du fragmentaire. . . . Elles invitent le spectateur «à recoller les morceaux», à intervenir pour donner un sens au procédé esthétique» (351). En effet, les ruptures mettent en valeur le processus théâtral où tout est joué et rien n'est vrai.

Toujours selon Pavis, la distanciation constitue un principe esthétique qui: vaut pour tout langage artistique: appliqué au théâtre, il concerne les techniques «désillusionnantes» qui n'entretiennent pas l'impression d'une réalité scénique et révèlent l'artifice de la construction dramatique ou du personnage. L'attention du spectateur se porte sur la fabrication de l'illusion, sur la façon dont les comédiens construisent leur personnage. Tous les genres théâtraux y ont recours. (125)

$\mathrm{Si}$, en effet, il s'agit du vaudeville, il pourrait signifier que le spectateur est amené à réfléchir davantage au contenu sérieux de la pièce. Le vaudevılle détruit même l'illusıon de réalité dans le contexte théâtral puisque tout est joué, imposant cette rupture entre la scène et le spectateur. Koffi Kwahulé ne veut donc pas que ce dernier soit obligé de se limiter ou de s'enfoncer dans une illusion dramatico-tragique qui pourrait être interprétée au premıer degré. Le sérieux du propos est confirmé dans la pièce par la présence répétée de l'Ave Maria. La comédie qui survient au début du dernier tableau met donc en évidence le côté joué de la représentation qu'il ne faut surtout pas prendre pour une imitation du réel. Il s'agit d'une fausse réalité dont l'objectif est de faire réfléchir sur sa vision du monde.

Étant donné que certains opéras sont de grandes tragédies, la musique classique revêt également toute son importance dans les didascalies. Dans la pièce, chaque tableau est associé à un Ave Maria en particulier: Ave Maria de Schubert égréné par la guitare de Cacho Tirao (Nativité), Ave Maria de Gounod interprété par Kathleen Battle (Messe basse), Ave Maria de Verdi, interprété par Renee Fleming (Prière), et Ave Maria de Caccini interprété par Ewa Izykowska (Confessions). Toutefois, dans une note aux praticiens, Koffi Kwahulé indique que les 
compositeurs et les interprètes ne sont mentionnés qu'à titre indicatif. Il s'agit ici de grandes vedettes de la haute culture, par opposition à la basse culture. De manière générale, il semble y avoir une contradiction entre cette culture musicale classique, celle des grands poètes compositeurs et des œuvres importantes de la musique religieuse et la basse culture, celle des toilettes, de la dégradation, de la dépravation, de l'abjection, de l'ordurier, de l'indicible, parce que trop osé et violent. Il s'agit donc des lieux de misère sexuelle mais également de tous ceux qui sont considérés comme les exclus de la bonne société, qu'il s'agisse d'immigrants ou de marginaux puisqu'ils font tous partie de la même structure paradigmatique.

Nous voyons qu'en transformant le frère étranger en Caïn, Koffi Kwahulé remet en question les catégories du récit biblique. Dhova ne tue pas son frère, comme c'est le cas au sein du meurtre fondateur de la civilisation chrétienne. Dans Ave Maria, le meurtre subit une transformation pour devenir la trahison qui détruit le couple et la famille, des valeurs et des éléments fondamentaux de la société chrétienne. Il s'agit alors d'une véritable subversion du mythe dans cette messe basse symbolique. Comme nous l'avons vu, la messe contient un côté indicible. Le bas du corps est synonyme d'interdit alors que le corps et le sexe sont proscrits. À ce sujet, nous pouvons renvoyer à saint Augustin, comme nous l'avons fait dans notre analyse de Bintou où la chair est interdite. Koffi Kwahulé traduit ainsi les peurs de la société qui craint que l'étranger, l'Autre, puisse détruire ses valeurs profondes en s'accouplant avec ses femmes. Cet étranger doit donc être gardé à distance pour empêcher cette situation de se produire et éviter qu'il n'enfante. Cette société n'admet pas ce type de mélanges qui entraîneraient des effets «néfastes» pour elle.

Avec son sexe, cet intrus représente le mal qui détruit la pureté du corps et de l'âme. L'auteur représente ainsi la peur fondamentale de l'Autre qui génère le racisme, voire la xénophobie, le signe même d'une société qui refuse ces mélanges. Cet Autre peut provenir d'un autre pays, d'une autre culture, d'une autre origine ethnique ou être considéré comme un 
marginal. Il demeure une personne qui n'est pas socialement acceptée. Cet Autre est toujours synonyme d'exclu, peu importe la manière dont il s'incarne. Koffi Kwahulé nous propose un théâtre qui ambiguïse toutes les valeurs sûres associées à cette société catholique, ce qui lui permet de mieux les critiquer.

Dans ce chapitre, nous avons tenté de démontrer comment Koffi Kwahulé s'appropriait les mythes et les subvertissait en leur donnant de nouvelles significations. Nous avons étudié cette désacralisation des mythes dans les cadres spécifiques de certaines réalités contemporaines. Dans le chapitre qui suit, nous avons regroupé trois autres pièces-Big Shoot, Blue-S-cat et Misterioso119 qui, comme nous le verrons, sont en fait des cérémonies du pouvoir qui portent davantage sur la mythologie de la victime dans des cadres plus abstraits. 


\section{CHAPITRE 3: LA MYTHOLOGIE DE LA VICTIME}

Dans le chapitre précédent, nous avons abordé la question de la réécriture des mythes et avons tenté de démontrer que, dans tous les cas, les formes de subversion étaient évidentes dans les pièces Cette vieille magie noire, Bintou et Ave Maria, où il s'agissait de trois réalités contemporaines facilement identifiables. En effet, il était question du racisme dans Cette vieille magie noire et de l'immigration dans Bintou et Ave Maria. Dans les pièces que nous analyserons dans ce troisième chapitre, Big Shoot, Blue-Scat et Misterioso-119, il nous semble que la réflexion s'exprime à partir d'allégories moins ancrées dans la réalité d'aujourd'hui.

Nous analyserons icı les rites de pouvoir et la mythologie de la victime qui seront marqués par la violence, puisque le viol, le meurtre, la trahison et l'agression jouent un rôle central dans ces cérémonies, mais où les références à une réalité extérieure (comme ce fut le cas dans le chapitre précédent), ne sont pas clairement reconnaissables. Il s'agit plutôt d'allégories qui concernent des victimes quı ne sont pas nommées et qui sont des voix scéniques sans identité extra-théâtrale. Dans Big Shoot, la victime est construite par le bourreau, qui l'affuble même d'un prénom arbitraire. Dans Blue-S-cat, on ne sait rien de l'homme qui est tué dans l'ascenseur. Dans Misterioso-119, tout ce que l'on sait de la victime, c'est qu'elle est intervenante en théâtre dans une prison pour femmes.

Nous avons porté notre choix sur ces pièces car elles dévoilent toutes la manière dont Koffi Kwahulé représente les différentes relations de pouvoir, où les victimes et les bourreaux en puissance se heurtent dans des corps à corps qui indiquent inévitablement des rapports de domination et de soumission. À ce stade, il est intéressant de noter que ces questions ne sont pas spécifiquement liées au racisme ni aux classes sociales. Elles symbolisent plutôt des paradigmes qui dépassent les conflits individuels pour cerner les structures profondes de tous les 
comportements haineux.

\subsection{Le bouc émissaire et les rituels de pouvoir dans Big Shoot}

L'analyse des pièces de Koffi Kwahulé révèle une parenté certaine avec l'œuvre théâtrale de Jean Genet, en ce qui a trait aux rituels de pouvoir. Les Bonnes de Genet, par exemple, possède un aspect sacrificiel qui s'inspire des rituels de la messe catholique et qui sont directement liés aux identités sexuelles des personnages mis en scène. Les jeux de pouvoir s'établissent donc par l'intermédiaire de ces rapports sexuels. La violence, qu'elle soit physique ou psychologique, ainsi que la mise en scène des rapports érotico-sexuels, surgissent au sein de ces relations de pouvoir.

Nous pouvons tracer des rapports semblables chez Koffi Kwahulé pour mieux déterminer la nature des rituels de pouvoir qu'il élabore entre les personnages et le milieu dans lequel ils évoluent. La pièce Big Shoot nous apparaît comme l'une des plus significatives à cet égard étant donné que le jeu de pouvoir culmine par la mort de l'un des deux protagonistes. Dans sa réécriture des mythes, l'auteur semble s'inspirer du premier meurtre fondateur perpétré par Caïn, le premier fils d'Adam et Ève, qui tua son frère Abel.

La pièce constitue un face à face entre un bourreau, «Monsieur», et sa victime «consentante», qui semble se prêter volontiers au jeu sadique du bourreau. Il s'agit du spectacle de la mort joué devant un public avide de sang. En effet, Monsieur est une sorte de tortionnaire qui accueille, devant les caméras de télévision et les spectateurs en studio, un invité dont le sort est connu d'avance. Il lui fabrique une histoire, lui impose un crime odieux, le soumet à un interrogatoire, lui inflige de multiples sévices, l'insulte sans arrêt et lui trouve même un alibi. Pourtant Stan, la victime, ne bronche pas, s'efforçant de dire ou de faire ce qui lui est demandé par celui qui mène l'événement, un maître du jeu qui se laisser aller au gré de ses pulsions. Stan aurait donc violé et tué une femme, ce qui se conclura par l'exécution du coupable par le 
bourreau.

Le texte n'est organisé ni par tableaux ni par scènes. Il s'agit d'une succession de répliques ininterrompues entre les deux hommes, où l'identité de celui qui parle doit être devinée par le lecteur ou le metteur en scène, d'après les détails qu'il peut repérer dans le texte. Il faut cependant souligner qu'il n'est fait mention d'aucune indication relative à l'âge et aux véritables noms des protagonistes, et que nulle description de leur allure physique ni de leur tenue vestimentaire n'est précisée. Écrite en l'an 2000, cette pièce est fort différente de Cette vieille magie noire, de Bintou et d'Ave Maria, trois textes où le lieu était identifié-respectivement la ville de New York, la banlieue d'un grand centre urbain européen francophone évoquant Paris et l'univers religieux fortement typé de la ville de Québec — et où l'auteur attribuait aux personnages des signes identitaires précis, tels que la couleur de la peau et des références culturelles clairement identifiables.

Le titre même de la pièce, Big Shoot (en anglais), renvoie à une expression polysémique, dans un registre populaire et familier de cette langue. Ambiguë par ses multiples lectures possibles, l'expression «big shoot» peut signifier une situation où quelqu'un devient une cible facile, où une personne — un pigeon—est prise au piège dans un guet-apens. La situation est donc sans issue pour celui qui, telle une proie, est traqué et tente d'échapper à un prédateur qui cherche à l'éliminer. Le titre peut également faire référence à la possibilité d'abattre quelqu'un avec une arme à feu (shoot)—Monsieur possède une arme à feu avec laquelle il menace Stan. Il peut également évoquer l'injection d'une drogue par intraveineuse ou le fait d'avoir une relation sexuelle rapide. Nous pouvons également associer le titre à une séance de photo de mode ou à un tournage de film.

Dans cette multiplicité de sens possibles, il est toujours question d'une confrontation entre deux personnes, une lutte qui pourrait symboliser le rapport de domination et de soumission ou, même, un état d'abjection symbolique relativement à l'autre. Ce titre devient donc très signifiant 
lorsqu'on examine l'accumulation d'images de pouvoir évoquées par la situation de Big Shoot.

Big Shoot est l'illustration d'une relation de pouvoir très particulière construite entre un homme, appelé «Monsieur», qui joue le rôle de bourreau, et sa victime consentante qu'il a baptisé Stan. Cette notion de victime consentante renvoie à la théorie de la victime émissaire, telle que formulée par René Girard dans Le bouc émissaire, où l'illusion persécutrice est partagée entre le bourreau et la victime. La société judéo-chrétienne serait la seule à considérer ces victimes comme innocentes. Girard cite en exemple Job, le Christ et les martyrs qui ont tous été persécutés. La notion de bouc émissaire renvoie au rite expiatoire du Yom Kippour (le Jour du Grand Pardon) chez les Hébreux. Deux boucs étaient choisis, l'un devait être sacrifié et l'autre était envoyé dans le désert: «Aaron impose les deux mains sur la tête du bouc vivant: il confesse sur lui toutes les fautes des fils d'Israël et toutes leurs révoltes, c'est-à-dire tous leurs péchés, et il ies met sur la tête du bouc; puis il l'envoie au désert sous la conduite d'un homme tout prêt. Le bouc emporte sur lui toutes leurs fautes vers une terre stérile» (Lv 16.21-22). Ainsi, au terme de ce rituel également pratiqué par Jésus, les prêtres et le peuple pouvaient recevoir l'absolution.

La victime n'est pas choisie au hasard mais doit en posséder certains traits ou caractéristiques. En effet, dans sa discussion sur le bouc émissaire, Girard rappelle que les victimes peuvent être accusées de tous les maux, qu'il s'agisse de la peste, de mauvaises récoltes, de parricide, d'inceste et d'empoisonnement moral ou physique de la communauté touchée. «Ces accusations sont caractéristiques de la façon dont les foules déchaînées conçoivent leurs victimes» (40).

Et qui sont donc ces victimes des persécutions? Le bouc émissaire fait partie de la minorité qui, souvent, devient la cible des violences mais rarement sa source. Dans La violence et le sacré, Girard insiste sur le fait que, en ce qui concerne le sacrifice humain, les traits victimaires désignent des personnes anormales:

Il y a les prisonniers de guerre, il y a les esclaves, il y a les enfants et les adolescents 
non-mariés, il y a les individus handicapés, les déchets de la société. . . .

On n'a donc affaire, pour l'instant, qu'à des catégories extérieures ou marginales qui ne peuvent jamais tisser avec la communauté des liens analogues à ceux qui lient entre eux les membres de celle-ci. C'est tantôt leur qualité d'étranger ou d'ennemi, tantôt leur âge, tantôt leur condition servile qui empêchent les futures victimes de s'intégrer pleinement à cette communauté. (24-25)

Une fois la victime identifiée, le crime qui lui est reproché devient secondaire pour la foule. Pressée de se débarrasser du fléau qui l'accable, la société réclame la mise à mort du bouc émissaire. Ceci, afin de revenir à la normale et, par le fait même, de sortir de la crise par le mécanisme sacrificiel. La communauté peut de nouveau resserrer les rangs, dans une nouvelle unification qui s'effectue autour de la victime.

Les pièces de Koffi Kwahulé s'articulent autour du paradigme des exclus, quels qu'ils soient. Dans Big Shoot, il n'y aucune indication sur l'identité de Stan. Il ne s'agit que d'un prénom arbitraire choisi par le bourreau pour mieux souligner son rôle de victime et le sacrifice auquel il est soumis afin de l'empêcher d'adhérer à cette communauté.

Notre analyse doit également tenir compte des indications scéniques qui identifient le lieu où se déroule toute l'action de Big Shoot. Selon Kwahulé, il s'agit d'un lieu aseptisé, nettoyé de toute trace d'humain, un lieu destiné aux animaux: "Une cage de verre. Peut-être un abattoir. Sans odeur ou trace de sang. Un abattoir excessivement propre, nettoyé à l'ammoniaque, clean. Peut-être une arène. En tous les cas, pas un ring. Surtout pas. Finalement une cage de verre. Carrée de préférence. Donc une cage de verre carrée, deux hommes» (Big Shoot 9).

Dans un premier temps, Koffi Kwahulé met en scène un rituel de mise à mort. Les deux hommes se trouvent dans la cage où Monsieur, le bourreau, doit tuer sa victime, Stan. Cependant, il y a une interaction importante entre les deux hommes qui, d'après l'auteur, forment un couple morbide, l'un ne pouvant fonctionner sans l'autre. La victime est, selon la perception du 
bourreau, une inspiration, voire une muse. Le bourreau ne pourra pas tuer sa victime sans que celle-ci ne lui donne envie de l'exécuter. De la même manière, le bourreau exerce une fascination étrange sur Stan, qui se laisse mener à sa mort sans protester, comme si la chose était inévitable et souhaitée, et que toute marche arrière était impossible. Dans Le bouc émissaire, Girard traite de la «collaboration mimétique des victimes avec leurs bourreaux» (94), une idée parfaitement illustrée par la relation qui se dessine entre Monsieur et Stan. «Big Shoot est un spectacle. Un spectacle de la mort. Un spectacle auquel qui veut y participe. Un spectacle sous forme de jeu où le gain n'est pas palpable. Gagner le droit de mourir. Une mort méritée. Une mort en public. Pour exister, enfin!» (Boëlle).

Il est important de souligner que le bourreau évoque également la présence d'un auditoire invisible qui assiste à ce jeu étrange de mise à mort cérémonielle. Il s'agit d'une illustration d'un rite culturel définissant cette société. Le texte suggère en effet que cet événement a déjà eu lieu à maintes reprises. Toutefois, ce rituel est nécessaire à la communauté, car «la culture née de la violence doit retourner à la violence» (Girard, Choses cachées 224), et que la mort est essentielle pour créer une nouvelle société.

Étant donné que la présence d'un public scénique est évoquée, cette confrontation devient, sur le plan théâtral, une mise en abîme. Le public scénique, et possiblement les spectateurs dans la salle, sont venus assister à ce spectacle pour satisfaire leur désir de voyeurisme, leurs envies morbides. Après que le bourreau ait abattu Stan d'un coup de pistolet, il présentera la balle au public qui signale son approbation par des applaudissements. Nous pouvons également deviner la fonction thérapeutique ${ }^{40}$ de ce spectacle pour les publics scénique et réel, puisque ce «sacrifice a pour fonction d'apaiser les violences intestines, d'empêcher les conflits d'éclater» (Girard, Violence 27). Dans la pièce, Monsieur insiste sur le fait que les spectateurs:

N'attendent que ça! Que je te fasse sauter la cervelle. Bang! Parce qu'ils viennent de

40. Cecı correspond à la fonctıon de catharsıs de la tragédıe arıstotélıcıenne 
loin, ces gens-là, de très loin, du bout du monde, souvent même à pied, tu m'entends, à pied du bout du monde jusqu'ici! Alors arrête tes conneries! Beaucoup de ceux dont tu devines les visages écrasés contre l'obscurité, là, ont économisé toute l'année sur leurs maigres soldes pour s'offrir ce moment... Et tu n'as pas le droit de te payer leur tête... Ta gueule, j’ai dit! (9)

Nous constatons que cette société a besoin de cette violence, de ce spectacle mortuaire pour assurer son équilibre affectif et psychique. C'est ce que décrit René Girard dans La violence et le sacré:

la violence fondatrice constitue réellement l'origine de tout ce que les hommes ont de plus précieux et tiennent le plus à préserver. C'est bien là qu'affirment, mais sous une forme voilée, transfigurée, tous les mythe d'origine qui se ramènent au meurtre d'une créature mythique par d'autres créatures mythiques. Cet événement est perçu comme fondateur de l'ordre culturel. (141)

Il est évident que le dramaturge porte un jugement sur la société actuelle qui comporte plusieurs niveaux de réflexion. Il remet en question la culture médiatisée par les moyens de communication moderne, par les guerres banalisées par la couverture télévisuelle et par les émissions de téléréalité où des gens anonymes et ordinaires exposent leur vie et leurs problèmes pour un peu d'argent, mais aussi pour satisfaire leur soif de gloire et de reconnaissance afin de devenir, eux aussi, des vedettes.

Koffi Kwahulé dénonce les violences de toutes sortes et le fait que le public jouisse de la misère des autres, une misère incarnée par l'abjection de la victime que nous voyons dans la cage de verre. Stan est donc celui qui incarne cet abaissement moral et physique, afin de satisfaire les désirs non-avoués du public. Le drame qui se déroule dans cette cage de verre constitue également une parodie du système juridique. Le procès se réduit à une forme de théâtre où le bourreau joue, en même temps, les rôles de juge et d'avocat. Inscrites dans cette mise en scène, 
les séances de torture se déroulent pour le pur plaisir d'un public avide de sensations fortes et de drames en direct.

Nous pourrions poser les questions suivantes: Est-ce que cette mise en abîme n'est autre chose qu'une incarnation théâtrale des pratiques qui se font régulièrement dans certains régimes politiques? Est-ce que ces pratiques qui, grâce à un miroir grossissant, sont renvoyées aux spectateurs par le dramaturge, n'ont-elles pas pour objectif d'aboutir à une catharsis? Nous pourrions répondre que cette catharsis permet un retour à l'équilibre jusqu'à la prochaine crise mimétique où, le besoin de sang et les désirs de spectacles violents des spectateurs seront résolus par un nouveau sacrifice. Il s'agira alors de la mise à mort d'un autre bouc émissaire lors d'une nouvelle crise mimétique.

Le récit qui déclenche la cérémonie de mise à mort est la suivante: le bourreau accuse Stan d'avoir tué une jeune femme en luı enfonçant un pique-feu brûlant dans le sexe et de l'avoir ensuite violée. La violence fondatrice imaginée ici est purement sexuelle. Il n'est pas question de la violence faite à une «Blanche» par des «Noirs», comme celle observée dans Les Nègres de Genet, où la violence a un fondement racial.

La vision de Koffi Kwahulé n'est pas de cette nature. Le dramaturge dénonce plutôt un appareil judiciaire qui produit des bourreaux et des monstres qui s'abreuvent de victimes innocentes. Nous voyons que la rencontre entre les deux hommes devient une métaphore des rapports issus de la colonisation de la période de postindépendance. Stan est dominé par Monsieur, un ancien petit fonctionnaire qui représente la figure d'une certaine classe de la bourgeoisie s'étant emparée des structures de la société coloniale en place, à la suite du départ des Européens. Ainsi les meneurs africains n'ont fait que prolonger la société coloniale. Dans cette période de décolonisation, l'esclave du colonisateur est devenu la victime de toutes les oppressions, et, surtout, du racisme de la part de son propre peuple.

Cette façon d'aborder les rapports entre l'homme et le monde renvoie directement à 
Frantz Fanon dans Les damnés de la terre. Fanon explique comment les bourgeois colonisés adoptent, après la décolonisation, le modèle hérité des colonisateurs pour la perpétuer. De ce fait, ils s'en servent pour opprimer leurs semblables et maintenir les privilèges qui étaient ceux de leurs colonisateurs. Selon Fanon, le pouvoir de «La bourgeoisie nationale, reprend les vieilles traditions du colonialisme, montre ses forces militaires et policières» (120). De plus, il précise que cette jeune bourgeoisie fait preuve d'un véritable racisme envers les siens. Il ne s'agit pas d'un racisme de mépris, comme celui qui émane des Occidentaux, mais bien d' «un racisme de défense, un racisme basé sur la peur» (158). Fanon estime que le racisme et l'oppression ne sont pas des problèmes liés à la couleur de la peau mais plutôt un rapport de nature économique entre les classes:

Le peuple, qui au début de la lutte avait adopté le manichéisme primitif du colon: Les Blancs et les Noirs, les Arabes et les Roumis, s'aperçoit en cours de route qu'il arrive à des Norrs d'être plus blancs que les Blancs et que l'éventualité d'un drapeau national, la possibilité d'une nation indépendante n'entraînent pas automatiquement certaines couches de la population à renoncer à leurs privilèges ou à leurs intérêts.

Dans Big Shoot, le bourreau se défoule sur l'opprimé, car il a peur que Stan se soulève et prenne la place de ceux qui, comme Monsieur, détiennent le pouvoir.

Le mot «Afrique» n’est mentionné que dans un seul passage, vers le milieu de la pièce, et cette allusion au continent renvoie à la dynamique actuelle de mondialisation dont le dramaturge se sert pour dénoncer une nouvelle forme de colonisation et de racisme basé sur le pouvoir économique, et qui se traduit par la domination mondiale d'un seul pays. Nous comprenons que Monsieur a effectué un voyage aux États-Unis et que c'est grâce à cette référence que nous pouvons comprendre un autre niveau de signification de cette pièce. Il s'agit d'examiner la nature des rapports actuels entre la culture américaine et l'Afrique. 
Monsieur fait l'éloge de New York, une ville condamnée à rester éternellement jeune. Il la compare au corps d'une femme offerte et séductrice. Il dénigre l'Europe qui, dit-il, se vautre dans son histoire, son passé et son titre de Vieux-Continent. Il ajoute que l'Afrique, le berceau de l'humanité, n'apporte rien de bon non plus:

L'Afrique elle, elle a vieilli avant le monde. Comme un enfant rachitique... Parce qu'elle est née trop tôt, avant terme, avant tout. Elle n'a jamais été jeune, l'Afrique. Elle est née, elle a vieilli. Comme un fruit qui pourrit sur l'arbre avant d'avoir eu le temps de mûrir... L'Afrique est tellement vieille qu'elle est tombée en enfance... Je parie que tu n'as jamais prié pour elle, Stan... I did, I often prayed and mostly cried for Africa... But New York! Ah, New York!... If the whole world could be New York'

Tout au long de la pièce, il existe un fragile équilibre entre les deux protagonistes, Monsieur ayant le pouvoir de «sauver» Stan en décidant de ne pas l'exécuter. Les deux hommes discutent de tricot, de femmes et des États-Unis, comme si de rien n'était. Le bourreau lui rappelle qu'il détient le pouvoir.

Pour sa part, René Girard traite également de la dynamique entourant le sacrifice rituel et son rapport à la victime. «Il est criminel de tuer la victime parce qu'elle est sacrée... mais la victime ne serait pas sacrée si on ne la tuait pas» (Violence 9). C'est justement ce dont il est question dans Big Shoot, une pièce où la mort d'un homme se prêtant au jeu du bourreau est attendue et espérée, dû au caractère sacré de la victime. C'est à partir de cette violence fondatrice jaillie du tumulte originel, que nous verrons comment le mythe de Caïn et Abel est transformé, alors que l'abjection devient le système de relation mis en place par le bourreau.

La représentation de cette violence est un élément fondamental de la poétique théâtrale de Koffi Kwahulé, dans laquelle le Mal et le pouvoir se rencontrent. En avril 2008, lors d'un colloque qui lui était consacré, il avait déclaré: «Mes premières pièces étaient des démonstrations 
qui avaient une démarche politique. Je veux penser autrement la tragédie aujourd'hui» (Poétique Politique). Cette phrase nous renseigne sur les intentions de cet auteur dont l'œuvre plonge au cœur de la violence archétypale. Celle-ci ouvre la voie à un nouvel ordre du monde, au sein même de la civilisation judéo-chrétienne, un univers dans lequel les figures mythiques de Caïn et Abel font directement allusion aux frères de la Bible, au moment où Monsieur en discute en anglais avec Stan dans la pièce:

Then the Lord said to Caïn, «Where is your brother Abel?» «I don't know», he replied. «Am I my brother's keeper?» (Big Shoot 26)

Monsieur et Stan sont donc des projections de Caïn et d'Abel, puisque le premier tue symboliquement le second lors de cette cérémonie mise en scène devant public. Stan représente ici la victime qui n'est pas vraiment coupable mais plutôt «nécessaire», la victime archétypale essentielle au bon déroulement de la cérémonie et dont le but est d'assurer la continuité de cette culture. Nous pouvons expliquer le «succès» relatif de cette mise à mort en citant René Girard qui traite des victimes de rechange. «Il n'y a rien à « expier ». La société cherche à détourner vers une victime relativement indifférente, une victime « sacrifiable », une violence qui risque de frapper ses propres membres, ceux qu'elle entend à tout prix protéger» (Violence 13).

C'est justement pour sauvegarder cette société médiatique qui regarde le spectacle, mais également pour sauver la société dans son ensemble que ce geste devra être posé. Lorsqu'il parle de tromper la violence en permettant à cette dernière de bénéficier d'un exutoire pour se réaliser et, ainsi, de permettre le bon fonctionnement de l'ordre établi, Girard s'intéresse aux archétypes des frères ennemis que sont Caïn et Abel: «Caïn cultive la terre et il offre à Dieu les fruits de sa récolte. Abel est un pasteur; il sacrifie les premiers-nés de ses troupeaux. L'un des deux frères tue l'autre et c'est celui qui ne dispose pas de ce trompe-violence que constitue le sacrifice animal» 
(Violence 14).

Girard ajoute que cette absence d'exutoire sacrificiel fait en sorte que la jalousie de Caïn ne peut aboutir qu'à la violence. Contrairement à Abel, son frère ne sacrifie pas mais offre plutôt à Dieu les sommes de sa récolte. Dans la pièce de Koffi Kwahulé, il s'agit de métaphores tirées de l'Ancien Testament où Caïn tue son frère, annonçant sans ambages le geste que Monsieur posera à l'endroit de Stan, son frère «métaphorique». Le personnage de Stan devient à la fois le frère assassiné de la tradition biblique et le Christ martyrisé. Qu'il s'agisse du texte de la Bible ou de la pièce de Kwahulé, ces martyrs sont nécessaires, voire essentiels au salut de l'Homme afin qu'il soit possible d'envisager un lendemain.

Dans Big Shoot, c'est la société médiatique qui bénéficie du spectacle et du sacrifice et, ce, dans le but précis de maintenir l'ordre. Il en découle une soif de voyeurisme, qui se traduit par la présence de ces spectateurs invisibles venus assister à l'événement. Ceux-cı applaudissent le meurtre, participant directement au lynchage en soutenant les efforts du bourreau. Girard parle des «bienfaits» de cette violence au sein de la communauté: «C'est la communauté entière que le sacrifice protège de sa propre violence, c'est la communauté entière qu'il détourne vers des victimes qui lui sont extérieures. Le sacrifice polarise sur la victime des germes de dissension partout répandus et il les dissipe en leur proposant un assouvissement partiel» (Violence 18).

C'est pour cette raison que la répétition du spectacle morbide est si importante. Elle agit comme une soupape, un répit, qui permet justement à cette communauté de continuer à exister, à vivre et à se développer, dans une cohésion sociale qui durera jusqu'à ce qu'une autre crise se produise. L'exercice a une fonction de maintien de l'ordre. Girard résume ainsi ce qu'il qualifie d'efficacité sacrificielle: «Ce dénominateur c'est la violence intestine; ce sont les dissensions, les rivalités, les jalousies, les querelles entre proches que le sacrifice prétend d'abord éliminer, c'est l'harmonie de la communauté qu'il restaure, c'est l'unité sociale qu'il renforce» (Violence 19). Monsieur est donc l'exécutant de ce renouveau, même s'il ne parvient pas à expliquer 
véritablement le sens de son «travail» à sa victime: «Parce que tu crois que je comprends pourquoi, alors que je ne suis ni le maire, ni le gouverneur de cette cité, pourquoi vous êtes tous venus, sans que je vous y contraigne, vous êtes venus, les uns après les autres, me présenter votre nuque afin que j'y plante une balle et jette votre mort en pâture à ces gens?» (Big Shoot 39).

Bien que le bourreau ne saisisse pas toute l'étendue de son «œuvre», il tente de créer, «quelque chose de propre, de convenable, de sain» (38) à travers ce spectacle. Quelque chose qui lui permettra d'atteindre un renouveau artistique qui favorisera le renouvellement de cette société qui a besoin de ces sacrifices pour préserver l'ordre établi, mais également pour éloigner toute violence qui pourrait la contaminer. Cette façon de faire permet de maintenir un équilibre fragile, tout en évitant des débordements non souhaités. Par la mise à mort de Stan, le rituel de Big Shoot nécessite une explication pour justifier son existence. Même si Monsieur dit ne pas comprendre les raisons de sa présence dans ce contexte, il doit tout de même pousser Stan à trouver seul les raisons quı le poussent à accepter ce sacrifice ultime.

Pour justifier son geste, Monsieur doit aider Stan à inventer une histoire qui soit suffisamment dégoûtante. En tant que victime symbolique, Stan pourra alors accéder à la mort qui lui est réservée. La cérémonie de mise à mort ne pourra s'amorcer sans qu'il ne s'accuse d'avoir tué et violé une jeune femme. Comme nous l'avons vu, la violence fondatrice est d'ordre sexuelle. Tous les rapports de force que nous retrouvons dans la pièce entraînent des tensions. À travers des images et la violence sexuelle, elles sont représentées sous forme d'abjection.

Dans son essai Pouvoirs de l'horreur, Julia Kristeva traite de l'abject qu'elle associe à l'objet du «refoulement imaginaire» (14). Selon Kristeva, l'abjection peut s'exprimer sous de nombreuses formes dans la perversion, dans le rite de la souillure, dans l'exclusion et dans le péché chrétien. «Dans la modernité occidentale et en raison de la crise du christianisme, l'abjection trouve des résonances plus archaïques, culturellement antérieures au péché, pour rejoindre son statut biblique et même, plus loin, celui de la souillure des sociétés primitives» (25). 
L'abjection et la violence constituent des dynamiques récurrentes chez Kwahulé où les symboles de domination et de soumission abondent. L'homme y adoptant même des comportements associés à ceux des animaux. Kristeva explique que: «L'abject nous confronte, d'une part, à ces états fragiles où l'homme erre dans les territoires de l'animal. Ainsi, par l'abjection, les sociétés primitives ont balisé une zone précise de leur culture pour la détacher du monde menaçant de l'animal ou de l'animalité, imaginés comme des représentants du meurtre et du sexe» (20).

Il faut rappeler le lieu où l'action de Big Shoot se déroule: une cage de verre complètement aseptisé et purifiée comme un abattoir. En retrait du monde réel, cet espace ainsi créé et dans lequel les spectateurs sont présents, permet au spectacle d'exister. Il s'agit d'un lieu médiatique qui existe en dehors de la société, et qui, justement, permet à cette dernière de continuer à se développer grâce à cet espace de débordement où l'abjection est permise.

René Gırard traite de la questıon du sacrifice en regroupant les victimes humaines et animales dans une même catégorie, parce qu'il n'existe, selon lui, aucune différence entre les deux. Cette affirmation cadre, avec beaucoup de pertinence, avec l'analyse que nous pouvons faire de ce lieu de mise à mort institutionnalisé en tant qu'abattoir. Il n'y est pas possible d'y retrouver de quelconques traces animales ou humaines. Il ne s'agit donc pas d'un lieu référé comme tel, mais plutôt d'un lieu sans nom qui accueille des combats symboliques. Le dramaturge ne tient pas à imiter la réalité, mais plutôt à démontrer qu'il s'agit d'un lieu complètement débarrassé de toute trace d'humanité. C'est pourquoi il nous est possible d'avancer qu'il s'agit d'un lieu de rencontre de deux forces opposées, celle du bourreau et de sa victime.

Nous pouvons également voir que cette relation morbide qui culmine par la mort, de même que l'ensemble du spectacle, ont une fonction thérapeutique au sein de cette société, permettant d'y préserver un équilibre vital. Mais à travers ses violences et ses humiliations attendues, réclamées et servies aux spectateurs, l'abjection y est totale. «L'abjection est une 
résurrection qui passe par la mort (du moi). C'est une alchimie qui transforme la pulsion de mort en sursaut de vie» (Kristeva 22).

Toujours selon Kristeva, «l'abject est la violence du deuil d'un «objet» toujours déjà perdu» (22). Dans Big Shoot, cet objet est le pouvoir, où plutôt la perte de ce pouvoir et de l'identité, une des conséquences de la colonisation. Bien que les pays colonisés aient pu retrouver leur «liberté» à la suite de leur accession à l'indépendance, le résultat est demeuré le même, comme nous l'avons vu chez Fanon.

Monsieur se considère comme un artiste qui a besoin d'une muse pour pouvoir créer une œuvre qui soit nouvelle, à l'image de Dieu qui a lui-même créé le monde à son image, selon la Genèse. Kristeva traite de l'abject qu'elle associe à l'artistique. Elle affirme que:

l'abject est pervers car il n'abandonne ni n'assume un interdit, une règle ou une loi; mais les détourne, fourvoie, corrompt; s'en sert, en use pour mieux les dénier. Il tue au nom de la vie ... il vit au service de la mort ... il réapprivoise la souffrance de l'autre pour son propre bien ... il rassoit son pouvoir narcissique en feignant d'exposer ses abîmes : c'est l'artiste qui exerce son art comme une « affaire »... La corruption est sa figure la plus répandue, la plus évidente. (23)

En plus de se prendre pour Dieu et d'avoir un pouvoir absolu, c'est à dire le droit de vie et de mort, le bourreau se considère comme un artiste qui peut accomplir son devoir, et exprimer son désir selon l'inspiration du moment. Il est le sauveur du monde, le seul a détenir le pouvoir. Et ce pouvoir, il l'exerce avec la parole qui méprise et injure Stan, tout comme il encense l'Amérique et dénigre l'Afrique. Dans ce contexte, l'emploi de l'anglais symbolise la suprématie de la langue et d'une certaine idéologie de domination associée aux États-Unis et à la mondialisation.

Pour exprimer sa domination sur Stan mais, surtout, pour imposer son pouvoir de meneur de jeu, le bourreau affirme que le nouveau modèle de terre promise est l'American Way of Life, 
une terre promise où tous les rêves sont possibles. C'est pourquoi l'anglais-américain constitue le gage ultime de sa supériorité. Ainsi, tout au long de la pièce, le discours de Monsieur—qu'il dialogue avec sa victime ou qu'il se parle à lui-même—passe de l'anglais au français sans raison apparente:

Non, l'anglais ça te donne une de ces pêches! ça [sic] bande, ça a la trique. Tu as remarqué, de temps en temps, moi, sans crier gare, je m'offre une phrase en anglais... [... . et hop! c'est reparti, je me sens rechargé; m'offrir une ligne d'anglais ça me booste. C'est pire que la coke, ce truc-là. Mais attention, faut ce qu'il faut, faut pas tout mélanger, faut savoir ce qu'on veut : je préfère me booster à l'anglais qu'à la coke. (Big Shoot 26)

Le bourreau affirme qu'il ne parle pas anglais mais plutôt américain, une distinction importante compte tenu de l'importance qu'il accorde aux États-Unis. Lorsqu'il se rend compte que Stan parle lui aussi l'anglais, il est étonné de la justesse avec laquelle il le parle. Mais le bourreau n'apprécie pas de se faire dire qu'il a un «vrai accent bien compact» (27) par sa victime. À la fin de la pièce, au moment d'exécuter Stan, la question de l'accent revient entre les deux hommes, comme s'il s'agissait du prétexte nécessaire pour tuer la victime. «Un accent compact et terrible»... Je t'en foutrais moi de l'accent!» (46).

Au-delà du viol et du meurtre de la femme, le prétexte de l'accent du bourreau semble être la raison qui fasse basculer Monsieur. Nous pouvons supposer que c'est parce que cette remarque s'adresse directement à lui et constitue une insulte. La victime n'a pas le droit d'agir de la sorte et cette phrase, somme toute banale, signale véritablement la fin de Stan, même s'il savait d'entrer de jeu qu'il courait à sa perte. Monsieur savait également que les pulsions de dégoût et de fascination provoquées par les propos de Stan l'inciteraient à commettre un meurtre rituel devant un public enthousiaste.

Koffi Kwahulé réécrit les mythes qui, dans le cas présent, est celui de Caïn et Abel. Ceci, 
dans le but d'y représenter une nouvelle société qui est celle du paraître, de l'image projetée et de la gloire instantanée. Une société où le spectacle de la mise à mort d'une victime—qui, sans être coupable, est malgré tout consentante et complice—constitue l'ultime représentation que l'on donne en pâture à des spectateurs avides.

\subsection{Le meurtre rituel dans Blue-S-cat}

Blue-S-cat met en scène deux personnages sans nom. Les protagonistes sont un «homme» et une «femme» sans que la voix à l'origine de chaque réplique soit identifiée. Il n'y a aucune indication concernant leur couleur, leur l'âge ou la nature de leurs rapports. L'auteur mentionne seulement qu'ils sont tous les deux «élégants». La pièce comporte 25 courts tableaux ${ }^{41}$ non titrés et ni numérotés, dont l'action se déroule dans un ascenseur qui tombe en panne. Le texte ne comporte aucune indication concernant lequel des deux personnages doit prononcer les réplıques, même si les didascalies fournıssent parfois quelques indications à ce sujet. Tout revient au choix du metteur en scène, qui doit distribuer les répliques afin que la tension progresse pour culminer par un dénouement qui correspond à la logique du dialogue. En effet, les événements nous font comprendre que les deux protagonistes sont coincés dans l'ascenseur pendant un certain temps. Terriblement angoissée par cet inconnu, la femme finit par l'abattre. Celui-ci est donc la victime de ce face à face imprévu, où il est mis à mort dans cet ascenseur en panne.

Le titre Blue-S-cat évoque d'emblée le jazz et le blues, et plus particulièrement le scat, une variante musicale dans laquelle l'improvisation et le rythme s'allient au son de syllabes saccadées qui ne sont pas des mots. Ces sonorités propres au scat rappellent des instruments tels la trompette ou le saxophone, ou encore, font en sorte que la voix devienne elle-même un instrument au son nouveau. La notion de «scat singing» est attribuée au musicien Louis Armstrong. Considérée comme un accident du jazz, elle est caractérisée par une improvisation 
spontanée. Le blues évoque également la misère et la solitude d'une manière souvent sensuelle puisqu'il peut faire penser aux corps qui s'enlacent. Au début de la pièce, on entend également quelques mesures de «What a Wonderful World» de Louis Armstrong, et dans les didascalies, l'auteur spécifie qu'il faut faire entendre «la version la plus ouatée» (Blue-S-cat 71) de cette chanson. Il souligne ainsi l'importance du choix d'une version qui contribuera à mettre à l'aise les passagers, dans le plus pur style de la musique d'ascenseur. Ceci afin de créer une coupure brutale, car c'est au moment précis où cette musique déraille que tout bascule.

Cet espace clos est un lieu d'enfermement, hors du temps et de l'espace, un lieu d'enfermement réel mais aussi symbolique, un espace intérieur, où chacun se retrouve avec son bonheur ou son indifférence. Le dramaturge spécifie: «Tous deux semblent heureux. Sereins tout au moins. / . . . / Ils semblent, bien que les corps soient relativement proches l'un de l'autre, ne pas se rendre compte/ne pas tenir compte de la présence de l'autre. Chacun est dans sa bulle, heureux» (71). Mais cet ascenseur peut tout aussi bien représenter un lieu de l'interdit où s'entremêlent gêne, malaise et désir, un endroit qui engendre une inévitable promiscuité non désirée par les passagers. Il peut enfin provoquer la peur et l'inquiétude, car il n'offre pas d'issue. C'est en effet la mécanique et la machine seules qui décident du fonctionnement de l'appareil. Les craintes qui accompagnent un voyage en ascenseur sont cristallisées par la présence de boutons d'urgence, d'une alarme et de systèmes de communication vocale ou vidéo destinés à faire face aux imprévus. Les pannes et les rencontres avec des étrangers qui en découlent font partie des aléas de tout déplacement en ascenseur. Pour un court laps de temps, les passagers acceptent de se placer volontairement en position de vulnérabilité et de devoir faire confiance à ceux qui sont présents dans l'ascenseur au même moment qu'eux. Les ascenseurs constituent à eux seuls un microcosme de la société, dont le fragile équilibre est perpétuellement remis en question par la présence de l'Autre et par d'éventuels problèmes d'ordre technique, qui peuvent survenir à tout moment. 
Dans Blue-S-cat, cet équilibre est rompu quand l'ascenseur s'arrête brusquement. Ce qui provoque un point de rupture. L'indifférence qui s'était installée entre les deux inconnus, est immédiatement évacuée lorsque la musique cesse, soulignant du même coup la panne qui vient de se produire. Au bien-être relatif du départ, succède le malaise, la crainte et l'angoisse, car ce couple qui n'en est pas vraiment un, devient prisonnier de la situation. L'homme et la femme ne se connaissent pas et ne se souviennent pas de s'être un jour croisés dans l'ascenseur ou dans l'escalier de service. Malgré cette proximité qui leur est imposée, ils demeurent deux étrangers pour qui toute communication semble impossible. L'absence de musique et de mouvement entre les étages impose le silence, un silence destructeur qui mènera au drame. Jamais, ils ne s'adresseront la parole directement. Leurs soliloques se croisent mais ne se rencontrent jamais. C'est donc au lecteur ou au metteur en scène de deviner qui prononce les répliques et de dégager le sens qui doit s'en suivre.

Comme nous l'avons vu, l'auteur ne donne aucune indication quant à l'identité de l'homme et de la femme. Nous ne connaissons ni leurs origines ni leurs descriptions physiques. Le seul élément qui les oppose, et qui transcende toutes les autres différences, est leur genre masculin et féminin. À travers cet anonymat, ils incarnent donc des figures archétypales de l'homme et de la femme et, par cette promiscuité des corps, les désirs et les pulsions sexuelles qui peuvent survenir à tout moment.

Du fait qu'elle ne contrôle pas la situation, la femme est très inquiète. Émotive, voire obsédée, elle se braque complètement. Elle a peur: peur de celui qu'elle ne connaît pas, peur de son désir qu'elle ne veut pas assumer, peur du refus de son désir par l'autre, peur d'être contrainte par l'homme de poser des gestes qu'elle ne souhaite pas. Elle songe à ce qui pourrait lui arriver de pire. Elle est convaincue qu'il la scrute, qu'il la déshabille des yeux et qu'il ne songe qu'à soupeser ses seins. À tout moment, elle s'attend à ce qu'il l'agresse à l'aide d'un quelconque objet, qu'il s'agisse d'un couteau ou de son sexe. Déjà le texte livre une ambiguïté inquiétante: «Il va le sortir. 
Court et trapu. Non. Long et effilé plutôt. Incurvé. Légèrement. Comme un croissant de lune. Il va le sortir.» (75). L'homme symbolise le danger que représentent l'autre sexe et l'étranger, celui qui peut avoir des manières plus barbares. «Il le tient contre sa cuisse. Sûr que c'est une poche sans fond. Tout le long de sa cuisse. Long avec une tête plate comme ceux des coupeurs de cannes de Cuba. Ou des Antilles» (81). Ces paroles évoquent l'Autre, un homme foncé, non européen, originaire de Cuba ou des Antilles. De plus, le nom de sa femme, Mélidésha, n'a pas une sonorité européenne.

Quant à l'homme, il est d'entrée de jeu plutôt indifférent à l'autre «passagère», coincée avec lui dans l'ascenseur. Son discours intérieur semble plus rationnel, cartésien. Il évoque des statistiques, des chiffres et des pourcentages, et parle d'ordinateurs. Il représente «le comportement rationnel des institutions modernes» (Ruprecht). En pensée, il s'adresse à maintes reprises à Mélidésha, qui semble être sa femme. Son discours intérieur n'a rien à voir avec la situation dans laquelle il se trouve, jusqu'au moment où il remarque que cette femme est plutôt jolie, mais sans plus. Il comprend la crispation de la femme dans les circonstances. Pour détendre l'atmosphère, l'homme lui sourit, mais sa compagne d'infortune ne répond pas à son sourire. Son discours évoque une communication silencieuse dans laquelle il s'adresse à elle à la troisième personne, comme si, l’observant de loin, il se faisait des commentaires à lui-même. «Parce que / elle a peur. / C'est normal que / elle ait peur. / Être là, dans cette situation avec un homme. / Parce que / si j'avais été. / J'aurais pu» (80).

À un moment, l'homme soupçonne même le désir de cette femme, sans en être tout à fait certain. «Mais quelque chose me dit que. / Elle me cherche. / Quelque chose me dit que. / Elle m'agiche. / Quelque chose me dit que. / Elle me tente. / Me trouve à son goût. / Me trouve quelque chose. / . . . / Mais je peux me tromper. / Je peux me planter» (89).

Blue-S-cat est donc une pièce sur l'impossibilité d'entrer en relation, puisque les deux protagonistes ne s'adressent jamais directement la parole. Conditionnés par leur vécu, l'homme et 
la femme réagissent chacun avec la somme de leurs propres expériences et préjugés, produisant une image fantasmatique l'un de l'autre. Jamais ils n'entendent ni n'écoutent ce que l'autre a à dire. Ils ne se parlent qu'à eux-mêmes. Les corps ne se rapprochent qu'au moment où la musique de Louis Armstrong se remet à jouer. Alors libérés de leur carcan par la musique, l'homme et la femme se mettent à bouger et à danser après que «L'ascenseur, apparemment sous la pression de la musique, explose, les parois disparaissent dans les cintres-ou dans les coulisses» (85). En s'effleurant, leurs corps vont finir par se rencontrer, à la manière d'une «comédie musicale hollywoodienne» (85). Cet espace chorégraphique constitue une soupape qui libère un peu de pression dans l'ascenseur, ainsi que le désir que la femme ne peut assumer. Il permet de libérer les pulsions sexuelles. Mais il ne s'agit que d'un moment qui agit comme un trompe-l'œil, à la manière des procédés cinématographiques. Cet intermède fonctionne momentanément mais, une fois la musique terminée, tous les deux reprennent rapidement leur rôle respectif. La femme se sent toujours plus menacée par cet homme qui pourrait bien avoir en sa possession un objet, ou quelque chose qui puisse la forcer à se soumettre à sa volonté, même si cet objet —arme? couteau? machette? sexe de l'homme?—n'est jamais précisé.

L'autre moment où une tentative de communiquer est partiellement réussie, est lorsque la femme raconte à l'homme une histoire avec des gestes. «Ce que ce n'est pas : pantomime ou langage des sourds. Elle raconte simplement sans les mots, avec des gestes «inesthétiques» comme une image de Cassavetes» (90). Encore une fois, il s'agit d'un intermède qui n'aboutit pas à une véritable communication, car l'homme éclate de rire, mettant ainsi brutalement fin à ce début d'échange qui émergeait du silence.

La femme, craignant d'être attaquée par cet objet que l'homme cache peut-être dans ses mains ou dans ses poches, se prépare au pire. «Parce que... parce que s'il se jetait sur moi là le poignard sous la gorge et qu'il l'exigeait... Je pourrais crier hurler... Mais à la fin... À la fin. Donc prévoir prévenir prévention» (91). Plus personne ne peut empêcher le drame de se produire 
lorsque, son obsession ayant atteint un paroxysme, la femme le frappe «dans les parties» pour, ensuite, s'élancer à sa gorge et le mordre jusqu'au sang, martelant son visage avec sa chaussure à talon aiguille. L'homme s'effondre en tendant sa main droite ouverte dans laquelle germe une rose.

Nous voyons comment la panne de l'ascenseur renvoie au bouleversement de l'ordre social établi. La pièce symbolise les origines du monde par la violence, inspirée de la mythologie chrétienne. L'homme et la femme incarnent les figures mythiques d'Adam et Eve. L'ascenseur, lieu de passage marqué par l'anonymat, s'ouvre sur l'universel afin de devenir un «lieu sacrificiel» (Ruprecht). Bien que les deux êtres ne peuvent ni communiquer, ni s'écouter, ni s'entendre, ils peuvent cependant s'observer, tels des prédateurs guettant une proie. Mais Koffi Kwahulé, en transformant «la femme en ange exterminateur» (Ruprecht) qui renvoie directement à la Bible au moment où Dieu fait sortir Israël du pays d'Égypte (Ex 12.21-37), fart en sorte qu'ils transcendent et dépassent cette réalité. Dans la dernière des dix plaies d'Égypte, tous les premiers nés mâles des Égyptiens furent tués par l'Ange exterminateur envoyé par Dieu, alors que ceux des Hébreux ont été épargnés. Lors de cette funeste nuit pascale, ces derniers avaient immolés un agneau par famille après que Dieu ait ordonné à Moïse d'agir de la sorte. En appliquant du sang de la bête sur les deux montants et le linteau de la porte de chacune de leur maison, les Hébreux ont sauvés leurs fils.

Dans la pièce, la femme incarne cet Ange exterminateur qui tue l'Autre, car il symbolise la figure de l'Homme qui, par le fait même, doit devenir cet agneau sacrifié. Koffi Kwahulé va même plus loin, traçant un parallèle entre les attentats du 11 septembre 2001 à New York, la chute des corps du haut des tours du World Trade Centre et cet agneau sacrifié. En effet, la femme réfléchit sur l'impossibilité de sortir vivante de cet ascenseur en panne, comme tous ceux qui ont été coincés dans les tours jumelles lors des attentats: «Parce que même en cas d'incendie ou de quelque chose comme ce qui s'est passé à New York, on l'a vu, on descend toujours plus 
vite avec l'ascenseur et même s'il arrive que l'ascenseur tombe en panne parce qu'il lui arrive aussi de bouder l'ascenseur, les gens préfèrent sauter par les fenêtres plutôt que d'emprunter les escaliers» (87).

Le corps devient alors le seul véritable instrument de communication, et donc de séduction, qui permet d'entrer en relation avec l'Autre. «Parce que ceux qui sont restés à New York dans les immeubles ceux qui n'ont pas eu d'autres choix que rester eh bien ils ont été vaporisés. Vaporisés. Vaporisés. Pas de corps à honorer. Et là impossible de faire le deuil» (88), clame la femme avec lucidité. Cette réplique fait dire à Virginie Soubrier que cette violence soustend toute l'œuvre du dramaturge. «Pour Kwahulé, au tournant du XX $\mathrm{X}^{\mathrm{e}}$ siècle, le corps noir, fragmenté et disséminé par le commerce triangulaire, devient le paradigme du corps moderne et le point de départ d'une interrogation sur la violence qui traverse le monde contemporain» (Improvisation).

Il est intéressant de constater que le soliloque de la femme sur la violence de la chute des corps est intimement lié au fantasme qui l'habite au même moment, à savoir la manière dont elle résıstera à l'homme s'il tente de l'agresser. La réplique suivante de l'homme, ou devrions-nous dire son monologue intérieur, a trait au jeu de séduction qui se met symboliquement en place, car, selon sa vision fantasmatique de la situation, il soupçonne que cette femme tente de l'aguicher et de le séduire. L'élément de son discours qui nous semble le plus révélateur est cette allusion à l'agneau qui renvoie à l'Ange exterminateur, comme si tout était en place pour le sacrifice. «Mélidésha me dit toujours / Tu es un agneau et / les agneaux ne comprennent rien aux femmes» (89). Ainsi, l'homme / agneau qui ne comprend pas les femmes, sera tué par la femme / Ange exterminateur, parce qu'ils ne peuvent entrer en relation qu'à travers ce rituel sacrificiel de mise à mort.

Bien que notre étude ne porte pas sur la mise en scène des pièces de Koffi Kwahulé, nous devons tout de même signaler qu'il a lui-même monté Blue-S-cat en 2006. Il a attribué le rôle de 
l'homme à un acteur «Blanc» et celui de la femme à une comédienne «Noire». Ce choix lié à la couleur de la peau peut-être interprété de plusieurs façons. Nous pouvons nous demander si cette différentiation supplémentaire, après le genre, engendre une nouvelle signification. A priori, il est possible d'imaginer que ce choix puisse être tout à fait arbitraire, mais il contribue cependant à accroître les différences entre les deux protagonistes.

Il nous paraît essentiel de tracer ici un parallèle avec l'auteur LeRoi Jones ${ }^{42}$ et sa pièce $L e$ Métro fantôme (Dutchman), qui raconte la rencontre entre un jeune Noir et une jeune Blanche. Dans cet autre lieu clos, le métro, le duel symbolise les préjugés des Blancs envers les Noirs, mais aussi l'assimilation volontaire du jeune garçon par la société Blanche. Comme dans Blue-S$c a t$, ce huis clos sera également fatal pour l'homme qui est lui aussi poignardé par la femme, l'ange exterminateur. Au début de la pièce, lorsqu'elle mange sa pomme, cette femme évoque Ève goûtant au fruıt défendu en compagnie d'Adam. Tous les deux sont alors chassés du jardın d'Eden par Dieu (Gn 3). Selon Geneviève Fabre, «le théâtre révolutionnaire de LeRoi Jones, par sa violence provocatrice, lance un cri de guerre contre la dramaturgie occidentale et pose les principes d'une nouvelle esthétique noire» (2: 1182). Nous pouvons donc avancer que la pièce de Koffi Kwahulé est construite d'une manière similaire, puisqu'elle montre des transformations de cette nouvelle dramaturgie contemporaine issue de l'Afrique.

La pièce Blue-S-cat peut être considérée comme une remise en question de la dramaturgie occidentale, car elle renverse le récit d'Othello par Shakespeare, l'auteur emblématique de l'écriture théâtrale européenne. En effet, dans ce classique du théâtre élisabéthain, Othello le Maure, poussé par Iago et la jalousie qu'il sème dans son esprit, assassine sa femme Desdémone, une Blanche. Lorsque Koffi Kwahulé a mise en scène ${ }^{43}$ sa propre pièce, la femme Noire tue

42. Everett LeRoı Jones (Amırı Baraka) est écrıvain, poète, professeur, romancıer et essayıste Noır amérıcaın Il est également reconnu comme un militant du mouvement afro-amérıcann. Tout comme Koffi Kwahulé, 1 est très influencé par la musique jazz.

43 Vour la théâtrographe 
l'homme Blanc qui s'est retrouvé avec elle dans l'ascenseur. Selon sa vision, l'enjeu des identités sexuelles devient central. En effet, dans la scène de danse, l'homme et la femme ne s'enlacent ni ne se touchent. Ils dansent ensuite chacun de leur côté, se déshabillant pour dévoiler des signes tangibles de travestissement. Elle cache un short de sport sous sa jupe, alors que lui, porte des bas résille et des porte-jarretelles. «On peut voir dans ce travestissement ... l'interchangeabilité des rôles de l'homme et de la femme» (Bérard). Ce choix de mise en scène interpelle le spectateur, car il énonce l'impossibilité de la rencontre-le déguisement étant simplement une tentative d'appropriation du corps de l'autre. Le vêtement masculin / féminin n'est, lui aussi, qu'un symbole des identités qui peuvent être renouvelées. Dans cette esthétique de violence, l'auteur montre que la violence engendre la violence et que les différences identitaires-homme, femme, Noir et Blanc-sont toutes interchangeables. Quand le jeu de travestissement est terminé, chacun remettra ses vêtements au moment où la musique techno s'efface pour reprendra sa place, comme si rien ne s'était passé.

Outre la musique techno, Koffi Kwahulé a également choisi de faire entendre celle du «Kol Nidra, prélude de l'office de Yom Kippour (le Jour du Grand Pardon) chanté en hébreu. Ainsi que le Blues annonçait l'intimité du couple, le Kol Nidra déclenche un profond bien être chez les deux protagonistes, les transporte sur le terrain de la mythologie biblique et ainsi, annonce le geste de mise à mort» (Ruprecht). Selon Koffi Kwahulé, il s'agit d'un «moment de repentance. L'espace de l'ascenseur devient le lieu de cette liturgie, durant laquelle, par cet accident mécanique, on revient sur soi, sur ses propres peurs» (Richon).

Comme nous l'avons vu, la forme de la pièce participe également au travestissement des mythes, notamment avec l'absence d'indication concernant celui ou celle qui donne la réplique. Tout comme la trame narrative, les échanges sont également inexistants. Livrés en alternance, les monologues intérieurs permettent cependant d'identifier qui, de l'homme ou de la femme, parle, en fonction des didascalies mais, également, grâce à la manière dont le texte est structuré. Alors 
que les soliloques de la femme sont regroupés en bloc, démontrant l'urgence de dire ce qui lui passe par la tête, ceux de l'homme consistent plutôt en de longs récitatifs incantatoires qui, de par leur disposition, ressemblent à des prières:

Mélidésha dit

Ça vaut le coup.

Mélidésha dit

Aucune raison de se laisser marcher sur les pieds.

Mélidésha dit

Faut continuer à y croire.

Mélidésha dit

C'est vital. (73)

Dans cette perspective, Koffi Kwahué réécrit les mythes désacralisés dans une approche moderne, juxtaposant certains éléments du monde contemporain et de la Bible pour donner un nouveau sens aux conflits qu'il a constatés au sein de la société. Dans Blue-S-cat, la condamnation est définitive. Il s'agit d'une vision très pessimiste puisque cette impossibilité à communiquer, incarnée par la mise à mort de l'homme, mine la société. Dans la vision du dramaturge, ce geste va se perpétuer dans une temporalité mythique, c'est-à-dire à l'infini, et deviendra, par là, l'essence même de cette mythologie urbaine où la rédemption est impossible.

\subsection{La messe et le meurtre rituel dans Misterioso-119}

La forme de la pièce Misterioso-119 ne correspond pas aux conventions de l'œuvre dramaturgique néoréaliste. Elle est plutôt une orchestration de voix (scéniques), comme s'il s'agissait d'un oratorio. Étant donné qu'elle ne comporte pas de didascalies ni de répliques alignées de manière traditionnelle, l'intervention du metteur en scène--qui doit travailler sur l'identité ainsi que sur la qualité de ces voix-est essentielle. La mise en scène participe donc à 
l'écriture de la pièce. Le texte de Koffi Kwahulé contient 17 tableaux dont les titres sont: I - Une enfance heureuse, II — Une vie sans fenêtre, III - En une fraction de regard, IV _Pom-pom girls, V — Et c'est pour cela que vous me tuez?, VI — Ces choses sales, VII — La pire des disgrâces, VIII — J'ai pensé à vous, VIX — Plus c'est sale mieux c'est, X — Visite, XI — Qu'elle périsse, XII - 119, XIII - La mauvaise, XIV - Maman, XV - Comme ça dans le train, XVI - Pardon et XVII - Eucharistie.

La pièce requiert la présence d'au moins quatre voix féminines, tout dépendant de la manière dont le metteur en scène orchestre ces voix. Il n'y a aucune indication quant à leur nombre ni à leur identité. Il ne s'agit pas de personnages, mais plutôt de voix qui forment un chœur éclaté. Et malgré qu'il n'y ait pas de logique narrative pour l'ensemble, il est cependant possible d'identifier certaines des voix, de par la récurrence de leur contenu et d'images qui reviennent comme des leitmotivs, permettant d'établir la continuité d'un certain discours entrecoupé par d'autres discours. À certains moments, on a l'impression que plusieurs voix parlent ensemble et que ces voix forment un chœur. Pour que ce chœur de femmes prisonnières puisse fonctionner et s'exprimer, il faut un minimum de deux voix auxquelles s'ajoute une troisième voix qui se distingue clairement des deux autres, une voix que nous appellerons la «prisonnière amoureuse». Il faut également une quatrième voix indépendante qui signale la présence de «l'intervenante artistique», aussi identifiée comme «travailleuse sociale» et «comédienne». Elle est l'invitée de l'extérieur, pour faire du théâtre avec les détenues et n'appartient pas, de prime abord, à l'univers carcéral.

Parmi les prisonnières, deux voix se distinguent plus particulièrement, de par la description des actes criminels qu'elles ont commis. L'une est identifiable du fait qu'elle raconte comment elle a tué son bébé en le plaçant dans un four à micro-ondes. L'autre purge sa peine pour avoir poussé son petit ami devant une rame de métro, parce qu'il avait osé qualifier ses gros seins de «pis de vache». L'identification est plus difficile pour les autres voix parce que les 
frontières entre ces voix sont souvent brouillées. Il est vrai que la récurrence de quelques leitmotivs nous permet d'identifier le discours de certains individus, sans pouvoir reconnaître les différents personnages pour autant. Il n'est, en effet, pas toujours facile de savoir qui «profère» ces images. Les leitmotivs qui permettent d'établir une continuité entre certains des discours, reviennent constamment pour souligner le fait d'avoir eu une enfance heureuse, d'avoir été avec un homme à la peau lisse comme un requin et de rêver de vivre à New York. Et bien que ces images exprimées ne permettent pas d'identifier les personnages, elles donnent cependant des indications sur la particularité de certaines voix en confirmant leur présence. De plus, les personnages n'ayant pas d'identité très précise, on pourrait même conclure que ces leitmotivs sont interchangeables et qu'ils contribuent à brouiller les pistes. À certains moments, le contenu de ces voix anonymes est très général, alors qu'à d'autres, les voix deviennent très spécifiques et identıfiables en établissant un dialogue—comme, par exemple, pour l'intervenante et la prisonnière amoureuse qui s'opposent aux autres détenues. Dans la structure, il existe parfois des oppositions qui permettent de savoir quelles voix semblent se donner la réplique. C'est notamment le cas dans l'exemple qui suit, alors que l'intervenante et la prisonnière amoureuse discutent entre elles:

Tutoyez-moi.

Non, non, c'est impossible. Le règlement...

Personne ne saura que / vous m'avez tutoyée.

Je ne tutoie jamais les participantes. C'est un principe. (34)

En outre, les différents contenus discursifs nous permettent de reconnaître les spécificités de certaines voix: lorsque les voix répètent la pièce de théâtre; qu'elles interviennent pour fournir une description historique des lieux; qu'elles racontent les récits de leur vie personnelle; qu'elles discutent au sujet de l'intervenante ou de la pièce qu'elles préparent. Il s'agit de multiples discours qui, dans leur globalité, agissent comme un chœur. 
Quelques tableaux proposent un court récit linéaire. Il s'agit de monologues qui racontent la vie de l'intervenante (II - Une vie sans fenêtre: «Regardez-moi, je ne suis pas d'ici, de dedans, je suis de dehors» (17)), celle de la prisonnière amoureuse (III — En une fraction de regard: «Je marchais dans le cloître, elle arrivait. Je ne l'ai pas vue, je ne l'ai pas regardée» (18)) et celles des autres prisonnières (VII — La pire des disgrâces et XIV — Maman: «Elle a vomi l'horreur que lui renvoyait le four à micro-ondes» (53)). Comme nous l'avons vu, il y a également quelques conversations entre l'intervenante et la prisonnière amoureuses (V - Et c'est pour cela que vous me tuez?, VIII - J'ai pensé à vous, XII- 119 et XVI — Pardon), qui démontrent une certaine progression du récit. Tous ces mouvements s'imbriquent les uns aux autres pour construire la structure générale de cette pièce composée de différents moments discursifs, qui ne sont pas nécessairement chronologiques.

Engagée pour monter une pièce de théâtre avec des détenues, la travailleuse sociale accepte, malgré la disparition inexpliquée des deux intervenantes qui l'ont précédée et d'une troisième qui se serait suicidée. Elle représente donc celle quı, à la différence des prisonnières, est libre parce qu'elle vient de l'extérieur de la prison et qu'elle peut s'en aller selon son gré.

C'est dans un univers carcéral féminin, lieu d'enfermement social par excellence, que se déroule l'événement scénique. Le tableau X — Visite nous permet d'en savoir un peu plus sur l'historique de ce lieu sur lequel une arène, une abbaye et un château (de la Vilaine) se sont succédés. L'endroit aurait également servi à l'Église pour y pratiquer des avortements sur de jeunes nonnes mises enceintes par le «Malin». L'Église a ensuite été chassée pour être remplacée par le régime de la première Terreur, sous lequel on a perpétré tortures et exécutions. Par la suite, l'édifice est devenu un couvent pour jeunes filles de bonnes familles. Il sera ensuite incendié, puis occupé par la marine nationale et, enfin, transformé en prison pour femmes. De par son histoire, ce lieu évoque un passé trouble, dans lequel la religion catholique et la violence physique sont étroitement liées. 
Koffi Kwahulé n'emploie jamais le mot prison dans le texte. Il est seulement mentionné en quatrième de couverture de l'œuvre, où l'on peut lire: «Dans un ancien couvent devenu prison pour femmes». Les prisonnières font souvent référence à «Mère Supérieure», qui est probablement la directrice de ce centre de détention, un élément qui renforce l'existence de ce lien entre la violence carcérale et l'Église catholique: «Tu veux peut-être que Mère Supérieure l'apprenne» (20). Le dramaturge met en valeur la nature transgressive de l'orientation même de la pièce. De plus, ce lieu—qui est la somme de toutes ces histoires—renvoie à l'autorité d'autrefois, dans une temporalité mythique, créant ainsi un lien où le passé et le présent ne font plus qu'un. Les rituels catholiques de l'ancien couvent et les rituels qui se pratiquent dans la prison se fondent également les uns dans les autres. Et bien que la structure du rituel catholique reste la même, sa nature est toutefois transformée par le dramaturge. L'essence même de la messe qui est représentée ici se retrouve alors dans une mise en scène transgressive, d'où la subversion du mythe. Par ailleurs, dans les deux cas, il s'agit d'un lieu de pénitence et de punitıon judiciaire. Le titre de la pièce annonce également une autre forme de transgression.

Misterloso-119 est une référence directe au jazz et à la pièce «Misterıoso», tiré de l'album du même nom de Thelenious Monk, enregistré live en 1958. Ici, le piano de Monk est remplacé par un violoncelle qui répète sans cesse dans l'auditorium 119 , situé à proximité du cloître. Nous pouvons y voir la rencontre entre le profane (le jazz) et le sacré (lieu voué à l'adoration de Dieu), qui annonce les stratégies dramaturgiques de l'auteur qui plonge l'Église et la prison dans cette transgression. Selon les prisonnières qui entendent inlassablement le son du violoncelle filtrer à travers les murs de la prison, il s'agit d'une «musique à fendre l'âme du silence» (15) et d'un «chant pour hâter la mort» (15). Ce chant annonce le début de la mise à mort qui sera déployée en présence d'un bouc émissaire, devenu figure messianique au sein du mythe chrétien, au même titre que Jésus, et qui, dans le rituel carcéral, est incarnée par l'intervenante. Il suffit de renvoyer à l'explication du bouc émissaire par René Girard, pour comprendre l'importance de cette 
fonction de victime expiatoire dans le rituel actuel.

\subsubsection{Le rituel en tant que théâtre}

Le texte révèle une mise en abîme, parce que la travailleuse sociale dirige les prisonnières dans une pièce de théâtre de l'auteure Elena Hebrayova. Koffi Kwahulé ne mentionne toutefois pas le titre de cette pièce mais nous devinons qu'elle gravite autour de certaines présences scéniques presque toujours invisibles et que cette œuvre dans l'œuvre contient à son tour, une deuxième mise en abîme racontée par la même narratrice. Les voix qui nous permettent de cerner la nature de cette pièce mise en abîme sont les suivantes. Il existe le «chœur» qui n'est formé que d'une seule voix narrative, celle de la prisonnière amoureuse, dont la fonction est de donner une description des événements qui constituent la pièce d'Hebrayova. Nous remarquons également la présence des «pom-pom girls», qui regroupent toutes les autres voix qui ne peuvent être distinguées, et dont le rôle est de donner à voir un spectacle musical. Les passages que le chœur récite sont indiqués en italique dans le texte. Nous verrons également plus loin que le récit qui est narré par le chœur dans la pièce d'Hebrayova est une fable mise en abîme, concernant l'histoire du Petit chaperon rouge. En effet, le chœur / narratrice raconte l'histoire d'une grand-mère et d'une fillette, vêtue de rouge, qui vont à un rendez-vous.

Présenté à partir du texte d'Elena Hebrayova, le spectacle, est également un amalgame de plusieurs formes théâtrales. Il s'agit d'un spectacle conceptuel où l'on retrouve «Théâtre, grande musique et pom-pom girls mélangés. C'est un truc à vous? Un nouveau concept, comme ils disent? C'est pour cela que / on vous a choisie, n'est-ce pas? C'est votre trouvaille à vous?» (25). Cet échange entre les voix au sujet du spectacle devient une discussion sur la forme théâtrale, qui permet d'aller encore plus en profondeur dans cette vision d'une mise en abîme remettant en question la fonction même du théâtre. Ce mélange des genres où les frontières ne sont plus claires, dérange les prisonnières. Pourtant, l'intervenante assure qu'il s'agit de quelque chose de 
très répandu.

La mise en scène de l'intervenante comporte deux éléments distincts. Pendant les répétitions, l'ensemble des prisonnières, qui compose le groupe des pom-pom girls, danse au rythme d'une musique. Il y a également le chœur qui, comme nous l'avons vu, n'est formé que d'une seule voix. À la différence du théâtre «classique», il ne subsiste que ce chœur ${ }^{44}$ solitaire dans cette mise en abîme. Le chœur symbolise une parole individuelle, à l'image d'une société de plus en plus individualiste et qui s'intéresse aux histoires les plus sordides véhiculées par les médias. Il s'agit donc d'un renversement du chœur traditionnel, car sa fonction actuelle étant différente, il ne peut donc plus être entendu comme par le passé.

Nous pourrions avancer que les conventions du théâtre occidental sont remises en question par la stratégie d'écriture de Koffi Kwahulé qui refuse, avec Misterioso-119, les conventions dramatiques traditionnelles. Nous pouvons en effet, le constater à travers la forme du texte—nombre indéfini de voix, absence d'identité—où seulement les tableaux, les structures discursives et les espaces typographiques servent de repères à la lecture. Cette remise en question se retrouve dans le rituel de mise à mort qui sera examiné dans la prochaine section.

\subsubsection{Le rituel de mise à mort}

La prisonnière amoureuse, qui déteste le fait d'avoir été sélectionnée pour incarner le chœur, rejette la pièce d'Elena Hebrayova qui lui a été imposée. Elle aurait préféré jouer le rôle plus spectaculaire de pom-pom girl. Elle n'hésite pas à dire à l'intervenante: «Mais vous êtes arrivée, vous m'avez désignée et vous avez dit Vous [sic] ferez le chœur. Toutes les autres, vous serez pom-pom girls, les Golden Girls. À moi seule je forme le chœur. Pourquoi m'avez-vous

44 Selon Pavis, «Depuıs le Théâtre grec, le chœur est un groupe homogène de danseurs, chanteurs et récitants prenant collectıvement la parole pour commenter l'action à laquelle ıls sont diversement intégrés Le chœur, dans sa forme la plus générale, est composé de forces . non indıvıdualısées et souvent abstraıtes, représentant des intérêts moraux ou politiques supérieurs 
imposé une telle idiotie?» (25).

La pièce d'Elena Hebrayova est écrite sous forme de scénario sans dialogues. L'unique voix du chœur n'est en fait que la voix du narrateur qui déclame un récitatif dans lequel il témoigne de ce qu'il voit, assis au café de la place du marché. Il s'agit d'une certaine vision du conte du Petit chaperon rouge ou «de l'idée qu'on s'en fait» (21). La fable met en scène une dame accompagnée d'une enfant habillée de rouge qui se dirigent vers la salle polyvalente pour y rencontrer quelqu'un. Sur place, un homme leur ouvre la porte et les fait entrer, heureux de les voir. Puis la dame repart seule. Inquiète, elle alerte aussitôt les gens de la place. L'un d'entre eux se rend à la salle polyvalente d'où il ressort seul. L'homme et l'enfant semblent avoir disparu. Impuissants, les gens ne savent pas quoi faire.

Il semble être question de disparition, d'enlèvement, de séquestration, de viol ou de meurtre. Mais il s'agit en fait du fantasme qui renvoie à l'idée de dévoration, issue de la tradition orale, et telle qu'on la retrouve dans les histoires de Perrault et de Grimm. Après avoir dévoré la grand-mère, le loup s'habille comme elle dans le but de dévorer la fillette qui doit lui rendre visite. Ce n'est plus l'homme qui mange l'animal mais plutôt l'animal qui mange l'homme. Étant donné qu'une forme de dévoration deviendra le point culminant d'un rituel de mise à mort qui met fin à la pièce, et que nous savons que Koffi Kwahulé a déjà établi un rapport de mise en abîme entre la messe catholique et le rituel carcéral, nous pouvons dire que le dramaturge trace un parallèle entre les épisodes de la vie, de la mort et de la transfiguration du Christ, à partir de la messe et du rôle joué par l'hostie, et le récit du Petit chaperon rouge dans lequel se retrouvent les mêmes éléments relatifs au mystère, à la disparition et à la dévoration du corps.

La pièce d'Elena Hebrayova évoque l'histoire du Petit chaperon rouge, qui est ici racontée comme un rituel criminel moderne. Dans Misterioso-119, Koffi Kwahulé reprend la même idée. À la différence que, dans sa pièce, ce sont les prisonnières qui finissent par dévorer l'intervenante, lors du dernier tableau intitulé Eucharistie, l'un des rituels de la messe catholique renouvelé par 
Koffi Kwahulé. L'auteur cerne le rituel de mise à mort à plusieurs niveaux. Dans un premier temps, il transgresse la vision catholique de la messe. Dans un second, il y a une vraie mise à mort rituelle de l'intervenante qui est sacrifiée par la prisonnière amoureuse et mangée par les détenues, une dévoration que la fable du Petit chaperon rouge annonce.

Cette fable populaire est le point de départ d'un vrai meurtre présenté comme la mise à mort rituelle de l'intervenante, moment charnière où cette idée de dévoration du corps du Christ est symbolisé par l'ingestion de l'hostie. Une rumeur laisse entendre que la prisonnière amoureuse a effectivement l'intention de la tuer, ce qu'elle finit par faire, l'ayant même prévenu de son geste:

Pourquoi voulez-vous me tuer? Vous le racontez autour de vous, à vos camarades, partout. Cela m'est parvenu.

Je ne vous prendrai pas en traître. Juste avant, je vous embrasserai devant les autres, je vous embrasserai sur la bouche, dans votre langue. (23)

L'idée même du baiser pour identifier celui qui va mourir, renvoie à la Passion du Christ et à la trahison de Judas. La prisonnière amoureuse désigne elle aussi sa victime en l'embrassant avant de la tuer.

Par la suite, elle offre à l'intervenante une bible ainsi qu'un dessin représentant un couteau. «Toute la semaine, je l'ai, d'une certaine manière, aiguisé. Pour vous» (32). L'intervenante allant même jusqu'à prendre la prisonnière dans ses bras et à la tutoyer, ce qui est strictement interdit par le règlement. Les deux femmes s'avouent qu'elles pensent souvent l'une à l'autre. La prisonnière explique également que: «on ne tue jamais que ce que l'on aime» (35) et qu'elle est elle-même une tueuse. Plus tard, l'intervenante lui offre une lime à ongles en or chromé en lui précisant: «vous en aurez besoin» (45). C'est dans le tableau XVI - Pardon, que la prisonnière la tue, c'est-à-dire la sacrifie. Pour l'intervenante, il ne s'agit pas d'une surprise, car elle se présente en tant que victime et que, soumise, elle s'attend à mourir comme le Christ sur la croix.

D'entrée de jeu, l'intervenante savait déjà que sa fin était proche. Elle ne parle pas de 
résignation, il s'agit plutôt d'un fait inéluctable. Le premier tableau se termine par le nettoyage des traces du meurtre de la victime précédente, tombée du septième étage, et dont la mort a été considérée comme un suicide par les autorités. Dans le tableau II - Une vie sans fenêtre, l'intervenante annonce sa mort proche, évoquant sa vie sans fenêtre, c'est-à-dire sans ami, sans mari et sans animal de compagnie. La prison constitue, à ses yeux, une sorte de refuge apaisant entre l'univers du dehors et celui du dedans. Elle s'interroge sur son sort mais résignée, elle assume pleinement son destin. Cette victime expiatoire accepte volontairement ce rituel, tout comme le Christ et Stan (Big Shoot) l'avaient fait avant elle.

La prison est un lieu qui reçoit les exclus, dans le cas présent, des femmes ayant commis les pires atrocités. Arrivée de l'extérieur de la prison, l'intervenante, qui est seulement venue pour partager son amour du théâtre, devient la nouvelle exclue, parmi les exclues de cette société qui mettra tout en œuvre pour l'éliminer, car sa présence remet en question l'ordre existant qu'il faut maintenir à tout prix. Son monologue est sans équivoque:

Une vie sans fenêtre. Peut-être la raison pour laquelle j'aime tant me retrouver ici, dedans. . . Regardez-moi, dans quelques jeudis j'aurai terminé mon contrat, les portes de dedans se seront refermées derrière moi, définitivement. Et ce jour-là, ce jeudi-là, je prie le ciel qu'il ne voit jamais le jour. Car ma seule fenêtre est ici, dedans. Regardez-moi, d'ici quelques jours je serai morte. (17)

C'est alors que le rituel de mise à mort débute. Tout juste avant de mourir, l'intervenante demande à la prisonnière si elle a avec elle sa lime à ongles. L'intervenante lui dit également de reprendre la bible qu'elle lui avait offerte en cadeau. «Aujourd'hui, je rejoins le dehors, pour toujours. Je ne veux plus revenir sur ce regard. Je suis épuisée. Je ne reviendrai plus ici, et je veux qu'on en finisse. Aussi vous ai-je attendue...» (58). L'intervenante invite la prisonnière à lui planter la lime dans une veine du cou. «Vous hésitez. . . Ramassez la lime et essayez de 
nouveau. Là, dans l'orémus ${ }^{45} \mathrm{du}$ sang, à l'endroit que vous dites si doux...» (59). Juste avant de poser son geste funeste, la prisonnière lui demande pardon.

Après le meurtre, la femme est démembrée pour être partagée et mangée par les prisonnières. Elles se distribuent alors les parties de son corps, comme elles l'avaient déjà fait avec les intervenantes précédentes. Ses viscères seront ensuite jetés dans le four crématoire afin de faire disparaître toute trace de sa présence. Il s'agit d'une référence directe à la Shoah, période pendant laquelle les Juifs sont, à leur tour, devenus les victimes expiatoires d'une idéologie génocidaire. D'ailleurs, le nom de l'auteure Elena Ebrayova évoque cette référence. La prisonnière amoureuse est prête à tout dévorer de sa victime, sauf le cœur. Elle préfère «le garder vivant dans ma chambre» (64).

Et si la prisonnière préfère garder le cœur plutôt que le manger, c'est pour continuer ce partage à un niveau symbolique:

La révélation chrétienne se réclame d'un transfert du sacrifice sanglant au domaine symbolique. Avec la figure du Chrıst, Agneau de Dieu qui incarne une victime innocente, parce qu'il se laisse immoler pour payer la dette des hommes, prendrait fin le schéma classique du bouc émissaire. Dans le mythe chrétien, la fondation sacrificielle de l'humanité est coextensive au sacrifice du «fils de Dieu». Le sacrifice du Christ rend par là même métaphorique tous les anciens sacrifices animaux. II désigne désormais le «sacrifice de soi». ${ }^{46}$ (Kilani)

L'ingestion du corps de l'intervenante peut être considéré comme une version renouvelée de la Cène, l'événement fondateur du christianisme incarné dans l'institution de l'Eucharistie. Les chrétiens de toutes les tendances considèrent que le dernier repas qui a réuni Jésus et ses disciples

45. Orémus sıgnifie «prions, mot prononcé dans les messes en latın par le prêtre pour inviter à la prière» (orémus) 46 Mondher Kilanı est professeur ordınaire d'anthropologie culturelle et sociale et directeur de l'Institut d'Anthropologie et de Sociologie de la Faculté des Sciences Sociales et Politıques de l'Unıversité de Lausanne (Suisse) 
le soir du Jeudi saint, a institué le sacrement de l'Eucharistie. La fraction du pain est un élément essentiel de l'Église.

Chez les catholiques, il ne s'agit pas d'un renvoi métaphorique. Les fidèles mangent vraiment le corps du Christ puisque le mystère transforme l'hostie en corps. L'hostie non consacrée est le pain. Lorsque le prêtre prononce les paroles de la consécration (ceci est mon corps livré pour vous), il signifie que l'hostie est devenue le corps du Christ ressuscité. C'est depuis ce repas collectif de la Cène que le pain, transformé en hostie, incarne le mystère catholique.

Lors de la Dernière Cène, Jésus a annoncé que le pain et le vin étaient associés à sa mort imminente et que son sacrifice seralt rédempteur. Dans la religion catholique, ce rite collectif est une communion mystique à laquelle participent tous les fidèles convaincus que le sacrifice de jésus représente l'amour infini de Dieu envers les hommes. Le Christ est «l'Agneau de Dieu qui enlève le péché du monde» (Jn 1.29).

Misterioso-119 transgresse l'essence même de la messe en la désacralisant et en éliminant le mystère catholique qui l'entoure. Cette déconstruction constitue la subversion même de la pièce. Dans la prison, lorsque la femme est tuée et mangée, l'Eucharistie perd alors sa valeur symbolique du fait de cette association entre ce geste et les images d'horreur inimaginables qu'il provoque. (Ce qui, bien sûr, dépend de la manière dont la scène est interprétée par le metteur en scène.) En mettant les prisonnières devant un corps bien réel qu'elles partagent et mangent, le dramaturge élimine ce mystère essentiel au rituel catholique. Ce qui constitue la transgression du rite qui, dans la continuité d'un temps mythique, continuera à se perpétuer.

En revanche, le corps ainsi partagé est l'interprétation littérale de la cérémonie eucharistique pendant laquelle le corps du Christ est transformé pour, ici, devenir le corps ensanglanté de l'intervenante. La représentation très troublante de l'Eucharistie par Koffi Kwahulé montre un événement qui est profondément transgressif lorsqu'il établit un parallèle 
entre cette cérémonie eucharistique et le cannibalisme.

En effet, le cannibalisme constitue l'un des grands tabous modernes, une pratique interdite par la société et qui, d'ailleurs, engendre partout un profond dégoût.

Dans la répulsion qu'elle suscite, cette transgression suprême éveille une peur venue du fond des âges comme si persistait, en chacun de nous, la trace d'une faute originelle. À tel point que, pour s'en affranchir, les populations «civilisées» ont souvent accusé d'anthropophagie les groupes humains qu'elles souhaitaient stigmatiser, en particulier au cours de la période coloniale où le «sauvage» était présumé cannibale. ${ }^{47}$ (Gilgenkrantz par. 1)

Cependant, l'abyme entre un cannibalisme tabou et une vision plus anthropologique de sa signification pourrait nous aider à mieux approfondir ce lien entre le rituel catholique et la mise à mort dans la prison de Misterioso-119. D'un point de vue anthropologique, le cannibalisme renvoıe actuellement à une autre façon de penser les relations sociales, selon Mondher Kilani. Ce dernier traite de la dimension symbolique et rituelle du cannibalisme actuel qui, sans que nous en soyons nécessairement conscients, existe cependant dans les farines animales, les greffes d'organes et les produits cosmétiques à base de déchets humains que nous consommons. L'analyse de Kilani «décrit en creux notre propre société capitaliste et rationnelle, qui semble fonctionner essentiellement sur le registre de la violence permanente. Une société qui a inauguré un cycle infini de destruction et de recyclage généralisé qui transcende les espèces et les ordres, et qui ne se préoccupe pas du sens qui nous lie à toutes ces entités».

Plusieurs formes de cannibalisme devenues légitimes, s'insinuent graduellement dans nos mœurs. Ces nouveaux rituels représentatifs des comportements actuels, que certaines pratiques

47 Simone Gılgenkrantz, est professeur émérıte de génétıque humaine à la Faculté de médecıne de Nancy (Clerey/Brénon) Dans cet artıcle, elle indıque que chez les Papous de Nouvelle-Guınée, cette pratıque a été observée lors des rites funéraires concernant des ennemıs Les hommes mangearent les muscles associés au symbole de la force, les bas-morceaux comme la cervelle et les viscères étant réservés aux femmes et aux enfants Dans Misterioso -119 , les femmes mangent le corps mais non les viscères 
cautionnent, provoquent un inéluctable glissement vers des rituels désacralisés et plus urbains, d'où une grande part de mystère est évacuée. Lorsqu'il entre dans les mœurs, le mystère n'est en effet plus nécessaire. Tout cela indique les premières étapes vers une nouvelle mythologie plus apte à cerner la nouvelle réalité des grands centres industriels modernes.

René Girard semble confirmer cette affirmation lorsqu'il estime que la disparition occasionnelle des tabous sociaux, des interdits comme le meurtre, et dont le cannibalisme est l'un de ses archétypes, permet ensuite de renforcer la cohésion et l'ordre social existant comme nous l'avons déjà vu.

Nous avons établi un parallèle entre l'Eucharistie catholique et la mise à mort de l'intervenante dans la prison, qui revit elle-même le parcours de la mise à mort du Christ. Après le meurtre de la femme, son corps est partagé et ingéré par les autres prisonnières, telle une hostie partagée par les fidèles lors du rituel de la messe. La transgression de l'Eucharistie est accomplie par le fait qu'il s'agit d'un vrai corps, ce qui désacralıse le mystère de ce rituel. Ce geste renvoie au cannibalisme, une pratique qui est traditionnellement défendue. Mais selon Kilani, ce cannibalisme existe actuellement sous différentes formes et entre graduellement dans les mœurs de manière inconsciente.

Koffi Kwahulé ne fait que confirmer une vérité dans laquelle le cannibalisme n'est plus considéré comme un mystère mais plutôt comme un rite faisant partie de mœurs, un rituel qui mettrait en valeur le geste fondateur de sa nouvelle mythologie urbaine. Nous voyons que cette mythologie urbaine met en lumière des rapports troubles, symbolisés par le récit du Petit chaperon rouge qui ferait également partie de nos mœurs, d'après la pièce d'Elena Hebrayova où il est question d'enlèvement, de disparition et de dévoration éventuelle par le méchant, le loup ou le «Malin». Koffi Kwahulé resitue cette fable dans la société actuelle et montre son rapport au sein de sa mythologie. 


\subsubsection{Les figures du Mal}

Dans cette vision d'une mythologie urbaine, Koffi Kwahulé semble avoir repéré certaines actions humaines actuelles pires que le cannibalisme. L'ingestion d'un corps humain est en effet considérée comme la transgression d'un tabou par la société, et n'est plus représentée comme un geste négatif. Il s'agit plutôt d'un acte quasi-religieux, un acte d'amour absolu où «on ne tue jamais que / ce qu'on aime» (59).

Comme nous l'avons vu chez Koffi Kwahulé, le cannibalisme dans le monde carcéral acquiert une connotation positive, tout en étant très violent. René Girard remarque qu'une logique semblable sert à justifier la pratique du cannibalisme rituel par les Tupinamba du Brésil, un peuple où la victıme est d'abord intégrée à la communauté avant d'être dévorée. Sa présence au sein du groupe et, par la suite, son ingestion, permet de renforcer la communauté. Lorsqu'elle est ramenée au village, la victime participe aux actıvités, se marie et peut même avoir des enfants. Au moment du sacrifice, un rituel d'évasion est même organisé pour mieux accuser le prisonnier quı devient alors le bouc émissaire «destiné à réincarner un héros mythıque» (Girard, Violence 413). Le parallèle entre l'intervenante qui doit être intégrée puis ingérée par les membres de la communauté carcérale et le prisonnier des Tupinamba est frappante. Au sujet de la future victime, Girard ajoute: «Pour la rendre apte à représenter adéquatement la victime originelle, il faut lui conférer ce qui lui fait défaut, une certaine appartenance au groupe, il faut faire d'elle une créature du « dedans », sans lui enlever pourtant sa qualité de créature du « dehors », cette extériorité déjà sacrée qui la caractérise essentiellement» (414).

Outre le cannibalisme qui se déroule dans le milieu carcéral de Misterioso-119, Koffi Kwahulé y dépeint la représentation de grands actes d'agression qui symbolisent les figures du Mal. Il s'agit de meurtres de nature collective qui sont dirigés contre des groupes d'innocents. Par exemple, il parle de l'extermination des Juifs qui ont péri dans les chambres à gaz lors de la Seconde Guerre mondiale, des gens qui sont morts dans les tours du World Trade Centre lors des 
attentats idéologiques du 11 septembre 2001 et des actes d'agression commis contre toutes les femmes.

Dans la pièce, la violence sexuelle, et la nature violente de tous les abus commis contre les femmes y compris le dépucelage, constituent d'autres grandes figures du Mal: «Tout ce sang, ça me rappelle la première fois, quand mon homme m'a étendue sur sa table de boucher, il est garçon boucher mon homme à moi, et qu'il m'a fendue et qu'il m'a fendue qu'il m'a fendue comme une pastèque» (16). Ces violences font partie des images récurrentes de Misterioso-119.

Nous pouvons également établir un rapprochement avec les corps pulvérisés dans les attentats du 11 septembre 2001, à New York, et celui de l'intervenante. De plus, en tuant la comédienne, les prisonnières tuent également l'auteure de la pièce, Elena Hebrayova, dont le nom évoque une origine juive. Dès lors, les prisonnières n'auront plus à participer aux répétitions et à la création du spectacle. Elles se sont en effet déjà mises en scène en accomplissant ce rituel de mise à mort collectif inscrit dans la Shoah qui est plus grave, selon le dramaturge, que le geste de cannibalisme.

Nous pouvons aussi dire que les attentats du 11 septembre 2001, qui ont tués plusieurs milliers de personnes, sont ici employés comme éléments de subversion sexuelle par rapport aux figures du Mal, à la suite de la diffusion à outrance de certaines images sur toutes les chaînes de télévision. Il associe cette destruction collective et l'horreur qu'elle suscité dans la mémoire collective. Le Mal est exacerbé et devient encore plus horrible lorsque l'auteur compare les avions à des sexes d'homme qui pénètrent des sexes de femme. Il associe les attentats idéologiques et une vision qui met en relief toutes les femmes abusées du monde, mettant en relief les viols qui sont utilisés comme une arme de guerre.

sous mes yeux qui n'osaient y croire, les corps des jumelles vaincus de jouissance se liquéfiaient dans le sang du sacrifice, au milieu des trompettes sardoniques de la poussière et du requiem de nos hurlements. Et 
j'ai pensé,

j'ai pensé,

j'ai pensé,

Qu'a fait l'Amérique pour mériter tant d'amour. Qu'a fait l'Amérique pour mériter

d'être l'hostie par laquelle l'humanité allait conjurer le millénaire qui s'ouvre... Parce

que

il ne s'agit que

de cela, d'amour... Toujours l'amour. Un amour si puissant que

il a submergé les sacrificateurs eux-mêmes... La poussière a bu le sang

La poussière est retournée à la poussière. L'holocauste a été accepté. (46)

Koffi Kwahulé évoque des images de viol de la Femme, qui deviennent le viol de l'Amérique commis lors des attentats de 2001 à New York, faisant ainsi le lien avec cette violence utilisée comme arme de guerre dans les conflits. Les grandes figures du Mal visent invariablement la destruction afin d'éliminer les collectivités lors des génocides.

Dans cette logique, le Mal devient toute cette méfiance et cette haine de l'Autre qui nous amène au rejet de celui qui est différent au sein des collectivités. Nous avons besoin de l'Autre pour assouvir nos désirs de destruction collectifs. Selon Myriam Vaucher, ${ }^{48}$

Peut-être en raison des violences du XXe siècle, mais peut-être aussi parce qu'il n'y a plus de sauvage, plus d'autre non-humain à manger, dans un monde où tout homme est voué à être reconnu comme humain. Le juif, le tsigane, le communiste, l'homosexuel, n'ont-ils pas été construits pour incarner l'autre dans un monde où il n'y aurait plus d'autre qui soit tout à fait étranger. . . .

Nous pourrions donc ajouter que ce Mal s'incarne dans ce besoin d'exterminer l'Autre et que cet

48 Myrıam Vaucher était présıdente de l'Assocıatıon internatıonale d'études médıco-psychologıques et relıgıeuses (AIEMPR) lors du congrès de l'Associatıon, quı se tenait en juıllet 2009 à St-Maurıce (Valais, Suısse) 
Autre inclut également tous les immigrants et les étrangers venus d'ailleurs.

Misterioso-119 est une réécriture mythique du cannibalisme dans laquelle l'objet consommé est une intervenante sociale assimilée à la figure du Christ. Il s'agit d'une interprétation littérale de l'Eucharistie où le corps est ingéré par les autres femmes. Bien que le titre de la pièce soit directement associé au Misterioso jazz de Monk, il s'agit également d'une référence au mystère catholique qui est ici transformé en rituel de mise à mort. Il est ainsi vidé de son «mystère», ce qui constitue la transgression même du mystère par l'association de l'Eucharistie au cannibalisme.

Comme nous l'avons vu, la victime est un bouc émissaire. Elle anticipe son sacrifice rédempteur qui fera d'elle cette victime expiatoire, selon Girard. Il suffit que la communauté la croit coupable pour qu'elle le devienne. C'est donc dire que les prisonnières reportent leur violence sur une victime innocente, une exclue, une personne à la fois en dehors d'elles et libres. Ainsi, la haine du groupe et le besoin d'exterminer l'Autre peuvent se focaliser sur cette victıme qui, à cause des accusations de la foule, en arrive même à se croire coupable.

L'auteur évoque également d'autres violences et d'autres fléaux, comme les grandes mıses à mort collectives. Il mentionne notamment les fours crématoires, les attentats du 11 septembre 2001 et les multiples violences sexuelles faites aux femmes. Toutes ces figures du Mal sont cependant beaucoup plus effrayantes que le cannibalisme, même si, de manière inconsciente, certaines formes de pratiques anthropophages seraient actuellement existantes dans notre société, selon Kilani.

La transgression ultime de Misterioso-119 est le fait de réduire tout ce rituel à un jeu théâtral où rien n'est vrai. Cette subversion, où tout est artifice, renvoie au domaine du fantasme du créateur, un produit de l'imaginaire qui se traduit par l'évocation d'une nouvelle mythologie urbaine et moderne, à partir de la mythologie traditionnelle judéo-chrétienne.

Dans ce chapitre, nous avons voulu démontrer comment les allégories mises en place dans 
Big Shoot, Blue-Scat et Misterioso-119 permettent l'élaboration d'une mythologie de la victime. Moins ancrées dans des réalités contemporaines comme celles du chapitre précédent, ces pièces, concernent des victimes qui sont des voix scéniques, des présences corporelles et des figures purement théâtrales. Ces victimes et leurs bourreaux entretiennent des rapports de domination et de soumission se terminant invariablement par la mort violente du plus faible. Bien que ces victimes soient toutes très différentes, elles font partie du même paradigme relatif aux comportements les plus violents de la société occidentale, et s'inscrivent toutes dans cette nouvelle mythologie urbaine qui émerge de l'écriture dramaturgique de Koffi Kwahulé. 


\section{CONCLUSION}

Au début de notre étude, nous avons tenté d'apporter quelques définitions associées aux notions d'africanité et au concept de «Noir» relativement aux différentes significations de la couleur de la peau dans les discours identitaires. Au départ, nous avons vu que l'Afrique est une notion problématique, car ses frontières géopolitiques internes sont le résultat d'un découpage territorial qui répondait à l'imaginaire des différents pays colonisateurs, sans que ceux-ci tiennent compte des réalités ethniques, linguistiques ou spatiales distinguant les habitants du continent. Pour cette raison, le concept d'africanité, est devenu, lui aussi, incertain puisqu'il regroupait des catégories culturelles, linguistiques et politiques difficiles à désigner par un seul mot et qu'il ne cernait nullement une réalité globale. Si nous poursuivons la logique des origines de cette termınologie, nous comprenons que la notion de «Noir» relève, dans un premier temps, d'une couleur de peau tout aussi imagınaire, puisque perçue par les habıtants européens «non-Noirs» qui avaient recours à cette désignation pour identifier une différence visible entre les Européens et ceux venus des pays colonisés ou anciennement colonisés. La notion de «Noir» s'est étendue pour devenir un objet de réflexion, de remise en question idéologique et l'expression d'un malaise au sein des populations «Blanches» des pays colonisateurs, attirant l'attention sur le fait que la notion de «Blanc», l'autre variable de cette pensée binaire, n'a jamais provoqué la même discussion.

Avec le passage du temps et l'évolution des théories identitaires, la notion de «Noir» associée à l'africanité, est devenue un concept de plus en plus problématique. Pour Aimé Césaire, Léon Gontran Damas et Léopold Senghor, le mouvement de la Négritude a été le point de départ d'un phénomène de résistance culturelle, par rapport au racisme envers les gens désignés par le mot «Noir» en France. Mais cela faisait également suite à la dévalorisation de ces individus par 
les autres Européens pour qui, le «Noir» incarnait le signe d'une personne qui leur était inférieure. Pour rectifier la situation, ces poètes de la Négritude voulaient valoriser les origines culturelles de ces populations diasporiques en chantant l'importance de l'identité désignée «Noire», mettant ainsi en relief les qualités d'une origine unique, associée à la métaphore de la racine.

Comme nous l'avons mentionné antérieurement, les œuvres du psychiatre et théoricien de la lutte anticoloniale, Frantz Fanon, proposent une réflexion fondamentale sur cette question. Fanon, qui s'est inspiré de Sartre (Réflexions sur la question juive) et des notions portant sur l'identité intersubjective de Hegel, a contesté cette approche de la Négritude, notamment dans Peau noire, masques blancs et Les Damnés de la terre. En effet, à la réflexion hégélienne sur l'intériorisation du regard de l'autre qui produit une identité intersubjective, Fanon y a ajouté la catégorie de race pour expliquer que le «Noir» se voit comme un «Non-Blanc», soit une négation $\mathrm{du}$ «Blanc», car il intériorise le regard dépréciatif que le «Blanc» pose sur lui.

Du point de vue théâtral, nous avons également proposé certaines pistes de réflexion en nous basant sur la pièce Les Nègres de Jean Genet, œuvre qui a beaucoup marqué toute une génération d'acteurs et d'auteurs dramatiques d'origines haïtienne, martiniquaise et guadeloupéenne. Cela s'explique surtout par le fait que cette création, mise en scène par Roger Blin en 1959, était très novatrice car, pour la première fois, le public français pouvait voir ces artistes Noirs sur la scène théâtrale parisienne. Dans son texte, le dramaturge s'interrogeait sur ce qu'était un «Noir» et de quelle couleur celui-ci devait être, puisque, selon Genet, l'identité était une entité qui se reflète dans les regards des autres jusqu'à l'infini, et qui est donc, impossible à cerner.

Ce sont ces transformations de la pensée identitaire qui trouvent écho chez une nouvelle génération de dramaturges d'origine africaine qui vivent ou travaillent en Europe et ailleurs dans le monde. Koffi Kwahulé fait partie de ce groupe auquel s'ajoutent notamment Caya Makhélé 
(République du Congo), Koulsy Lamko (Tchad) et José Pliya (Bénin). À travers leurs écrits dramaturgiques, ils proposent un renouvellement de la manière de représenter la notion d'Afrique au théâtre, d'un point de vue psychologique, culturel et social. Ils revendiquent tous une certaine africanité qui se base en premier lieu sur leurs expériences personnelles. En effet, Koffi Kwahulé propose une vision très personnelle de son africanité, déterminée par le fait qu'il soit d'origine ivoirienne et qu'il vive en France depuis plusieurs décennies. Il remet en question une vision monolithique de l'africanité et des questions identitaires et raciales qui en découle. Kwahulé est devenu l'une des figures de proue de cette réflexion identitaire des auteurs d'origine africaine qui vivent hors de l'Afrique. Son africanité est donc la manière dont il perçoit la société dans laquelle il évolue, ce que nous avons déjà appelé sa vision kwahuléenne du monde qui l'entoure:

Je n'ai jamais prétendu écrire un théâtre africain. Ce que les gens croient être un théâtre africain est une chose déjà morte. C'est une manière de réduire le Noir à l'épaisseur de ses propres fantasmes, de le fixer dans l'être, de lui refuser le devenir. Mon écriture n'est pas africaine. Elle ne porte que ma propre expérience, et elle est irremplaçable. Je ne peux témoigner que de cette expérience, de mon histoire, avec ses failles et ses faiblesses. (Kwahulé et Mouëllic 67)

Dans ce contexte, les écrits scéniques de Koffi Kwahulé nous apparaissent extrêmement importants puisqu'ils représentent cette fusion contemporaine entre une vision plus traditionnelle de l'Afrique et une vision transformée d'une africanité. En effet, nous avons voulu analyser ce théâtre «rhizomatique», qui capte la conscience de l'être exclu, par la manière dont son évolution est symbolisée au théâtre. Cette approche peut se résumer par une dramaturgie qui subvertit les structures de la mythologie judéo-chrétienne, mythologie renouvelée par le contact avec d'autres mythologies fondatrices héritées des immigrants.

Afin de pouvoir analyser avec plus de précision ces subversions du mythe fondateur qui donnent toute son impulsion au théâtre de Koffi Kwahulé, nous avons étudié quelques définitions 
du mythe selon Claude Lévi-Strauss, Roland Barthes et René Girard, et nous avons compris que l'écriture scénique de Kwahulé répondait à certains éléments de ces trois définitions.

Nous avons débuté notre étude à partir de trois pièces regroupées dans le deuxième chapitre: Cette vieille magie noire, Bintou et Ave Maria. Ici, nous avons analysé la manière dont Koffi Kwahulé s'est approprié les mythes fondateurs de la société judéo-chrétienne et comment ces mythes ont acquis de nouvelles significations. Notre étude a porté sur la subversion des mythes qui vise tout particulièrement l'émergence d'une nouvelle mythologie culturelle. Ces trois pièces, qui correspondent à une esthétique scénique néoréaliste, se déroulent dans des lieux géographiquement identifiables (boite de jazz, banlieue d'une grande ville, ascenseur d'un immeuble d'appartements). Elles sont également associées à des thématiques contemporaines comme le racisme, l'immigration et les tensions urbaines.

Dans le troisıème chapitre, nous avons ensuite analysé la mythologie de la victime à travers les pièces Big Shoot, Blue-S-cat et Misterioso-119 où, à la différence du premier groupe de pièces étudiées, la réflexion autour du mythe s'exprime à partir d'allégories qui sont moins ancrées dans une réalité contemporaine identifiable. De plus, à l'opposé des pièces du chapitre précédant, l'action se déroule dans des lieux clos. Les protagonistes ne sont jamais nommés et n'incarnent pas non plus de personnages. Contrairement aux conventions théâtrales néoréalistes, ils ne possèdent pas de psychologie et représentent surtout des voix scéniques, dont l'existence est purement théâtrale. De plus, les travaux de Girard, concernant la notion de bouc émissaire et de victime sacrificielle, nous ont permis de mieux comprendre le rôle de ces victimes au sein des sociétés.

À travers notre étude, nous voyons comment la poétique théâtrale de Koffi Kwahulé s'oriente vers différentes formes de métathéâtralité, nous permettant de reconstituer le nouveau monde mythique représenté dans ses pièces. En effet, dans un premier temps, nous constatons que le théâtre fait l'objet d'un commentaire explicite dans un discours métathéâtral. Les 
personnages donnent leur avis sur le théâtre en général ou sur différentes formes de théâtre:

«Théâtre, grande musique et pom-pom girls mélangés. C'est un truc à vous? Un nouveau concept comme ils disent?» (Kwahulé, Misterioso-119 25). Dans un deuxième temps, nous relevons une mise en abîme, c'est-à-dire la préparation d'un spectacle théâtral. Nous rencontrons des personnages ou, encore, des voix parlantes qui jouent différents rôles dans un théâtre à l'intérieur du théâtre, tels que le chœur, l'intervenante et les pom-pom girls, qui préparent un spectacle dans la prison de Misterioso 119. Un autre exemple de mise en abîme se retrouve dans Cette vieille magie noire, où le boxeur Shorty se transforme en comédien lors des répétitions du Faust d'après la pièce de Goethe. Ce sont là les meilleurs exemples de ce processus de réflexion sur le théâtre qui s’inscrit profondément dans sa poétique scénique et textuelle.

Toutefois, tous ces renvois à la distanciation métathéâtrale ne font qu'annoncer le fondement même d'une poétique dramaturgique dont les lois visent la création d'une nouvelle mythologie urbaine. Ceci par un processus de désacralisation de tous les rituels associés à la messe catholique, dans un contexte où tout est transformé en théâtre, y compris les rituels euxmêmes. Selon Richard Schechner, le rituel devient théâtre à partir du moment où la portée esthétique de l'événement s'impose sur sa portée transformative. ${ }^{49}$ En mettant en évidence l'esthétique du texte et les fonctions instables des voix scéniques, le rituel acquiert des qualités qui sont surtout théâtrales, ce qui diminue sa fonction transformative.

C'est pour cette raison que les meurtres sacrificiels, les confessions, les actes de traîtrise, les viols, l'adultère, la révolte des jeunes, leur répudiation des lois de la famille, les gestes violents, les chocs, l'esthétique victimaire, les rencontres intenses dans des lieux clos et tout le côté hautement spectaculaire de ces rituels sont privilégiés par l'auteur. Ces événements ont, bien sûr, une certaine portée transformative, à la fois sur le public, exposé à des situations qu'il

49 Cette idée de Schechner est développée dans «From Ritual to Theatre and Back: The Structure/Process of the Efficacy-Entertainment Dyad », Essays on Performance Theory 1970-1976 New York: Drama Book Specialısts, $197763-98$ 
méconnaît, mais aussi sur les personnages qui deviennent des incarnations du Christ—au féminin—et sur la victime expiatoire universelle. Toutefois, par la manière dont Kwahulé associe des figures scéniques aux figures centrales des rituels chrétiens-Jésus, le Christ crucifié, la Vierge et Marie-Madeleine-il privilégie une recherche sur de nouvelles formes théâtrales et sur des fonctions esthétiques repensées, une réflexion qui passe par l'art scénique reposant sur une poétique théâtrale, mue par une vision esthétique extrêmement transgressive. Il semble que la fusion entre le divertissement esthétique et la fonction transformative est telle, que ses œuvres deviennent tout à fait efficaces — où une transformation s'est opérée—dans le sens entendu par Schechner, qui s'inspire lui-même d'une notion anthropologique de Lévi-Strauss. Les œuvres de Kwahulé laissent donc une marque profonde sur le spectateur.

Nous renvoyant à de multiples mises en abîme, où des gens font du théâtre à l'intérieur du théâtre, le dramaturge nous rappelle constamment qu'il s'agit du théâtre. Pourtant, même si ses œuvres ne sont pas des rituels purs, où la fonction transformative est prioritaire, ses textes signifient, voire incarnent, une dynamique de changement de la société française. Ils montrent des ruptures culturelles permettant l'émergence d'une nouvelle mythologie urbaine, originaire des traumas qui frappent les habitants de ces nouvelles sociétés, le produit de la rencontre entre une africanité déstabilisée et une européanité qui l'est tout autant par les incessantes remises en question des sources de sa culture.

La poétique théâtrale de Koffi Kwahulé opère à plusieurs niveaux. À titre d'exemple, les protagonistes sont des présences très complexes qui se manifestent sous plusieurs formes. Certains d'entre eux peuvent même se matérialiser sous une forme réaliste à l'image de ceux de Cette vieille magie noire, Bintou et Ave Maria. Ces personnages vivent des expériences quotidiennes dans des décors auxquels le public peut s'identifier et où les gestes qu'ils posent vont souvent à l'encontre des attentes de leurs communautés. En revanche, les identités peuvent également être plus abstraites, comme celles de Big Shoot, Blue-S-cat et Misterioso-119. Dans 
ces cas, les individus ne sont pas clairement identifiés, les différentes voix possédant une fonction «supérieure» qui leur permet d'accéder aux actes rituels du domaine mythologique. La transgression du mythe naît du fait que l'auteur établit des parallèles entre la réalité de ces individus / voix scéniques et les rituels qu'il met en place autour desquels le sacré est dénoncé ou, encore, subverti, pour que les stratégies héritées du rituel religieux se déplacent vers des contexte sordides et destructeurs. La mort peut avoir une signification transformative dans le contexte de la pièce (comme nous l'explique Girard). Mais l'événement de mise à mort est inévitablement haineux, cruel, ancré dans la misère sexuelle, la pauvreté, le malentendu, les jeux de pouvoir, les rapports sadomasochistes et la guerre des sexes. Autrement dit, puisé dans toute la matière de la vie actuelle des grands centres urbains, telle que perçue par Koffi Kwahulé. La naissance de cette nouvelle mythologie urbaine kwahuléenne y retrouve toute sa logique.

Il faut dire que la question de l'adaptation des mythes n'est pas un processus employé uniquement par Koffi Kwahulé. Nous le retrouvons à travers toute la dramaturgie française: Sartre (Les Mouches), Camus (Caligula), Giraudoux (La Guerre de Troie n'aura pas lieu) et Anouilh (Antigone). Ces pièces, inspirées de la mythologie classique grecque, ont été créées en France, sous l'Occupation. Elles permettaient d'exprimer une forme de résistance fondée sur des images et des références que l'ennemi ne pouvait pas reconnaître.

De plus, dans ses écrits sur le théâtre, Sartre a évoqué le fait que l'être humain se libère en faisant les bons choix, tels que des «actes» de résistance. Ce sont ces choix personnels qui définissent son existence. Pour Kwahulé, qui s'intéresse à la mythologie judéo-chrétienne, ces choix n'existent pas, car les mythologies qui le hantent ne permettent pas de se définir grâce à cette liberté de choisir ou, encore, en agissant contre le «pouvoir». D’après l'optique de Kwahulé dans la mythologie chrétienne, les êtres humains sont soumis. S’ils se laissent martyriser et sacrifier, c'est parce qu'ils sont victimes d'une transcendance supérieure qui les dépassent et les écrasent. En revanche, dans le monde de Sartre et de sa vision existentialiste, cette transcendance 
n'existe pas. C'est l'homme même qui définit son existence par ses actes.

Dans ce contexte de remise en question de la notion de subversion autour du mythe, nous voyons que la fonction du mythe est différente chez Koffi Kwahulé. Si elle permet d'élargir le champ de sa réflexion théâtrale entre les pôles de la chrétienté et de l'africanité, c'est parce que le texte fondateur sur lequel il base son théâtre, est carrément remis en question et resitué dans le contexte autour des jeux de pouvoir qui ne sont pas nécessairement associés à une lecture biblique. L'auteur renouvelle tout. Il dénonce la situation des étrangers dans la société européenne et remplace les anciens mythes par une mythologie qui devient le récit fondateur d'une nouvelle culture française. Une culture qui est en train d'émerger dans des espaces de rencontre, entre des gens de diverses origines qui sont actuellement marginalisés par la société française. Nous pouvons aller encore plus loin pour ouvrir cet espace de réflexion.

Nous avons analysé son processus dramaturgique et dégagé de nombreuses rencontres mythiques, ancrées dans des contextes bibliques et rituels. En procédant de la sorte, Kwahulé met notamment en lumière certaines réalités rencontrées par les Africains en Europe. Se pourrait-il que sa nouvelle vision, formulée à partir de différentes sources et cultures qu'il a «ingérées», constitue une forme d'anthropophagie ou de cannibalisme littéraire, un procédé dans lequel les formes africaines, bibliques et allégoriques se trouvent digérées dans le but de créer une nouvelle culture signifiée par ce nouveau théâtre africain? S'agirait-il d'un acte théâtral anthropophage?

Il serait intéressant de prolonger l'image du cannibalisme si centrale dans Misterioso-119, la dernière pièce analysée, pour retrouver le lien entre cette idée et la théorie de l'anthropophagie littéraire. Notre questionnement s'inspire des écrits du poète brésilien Oswaldo de Andrade et de son Manifesto Antropófago (Manifeste Anthropophage). Selon François Laplantine, l'anthropophagie est «mise au service de la construction culturelle de la nation. . . La culture lusitanienne et plus encore les autres cultures européennes (en particulier la française), loin d'être rejetées, sont au contraire dévorées et assimilées. Le Brésil s'en nourrit mais les transforme, 
créant une culture résolument nouvelle» (82). Koffi Kwahulé annonce donc qu'un processus de transformation culturelle est en train de se réaliser en France. Oswaldo de Andrade fournit un concept qui nous permet d'ouvrir un nouveau champ de réflexion à partir du nouveau théâtre africain. 


\section{BIBLIOGRAPHIE}

Africultures. Fratries Kwhaulé : Scène contemporaine chœur à corps. Dir. Sylvie Chalaye et Virginie Soubrier. 77-78 (2009). Imprimé.

Anderson, Benedict. Imagined Communities: Reflections on the Origin and Spread of Nationalism. Rev. ed. London: Verso, 1991. Imprimé.

Appiah, Kwame Anthony. In my Father's House: Africa in the Philosophy of Culture, Oxford: New York UP, 1992. Imprimé.

Atlas des religions. Éd. Antoine Sfeir. Paris: Plon-Mame, 1994. Imprimé

Bachir Diagne, Souleymane, ed. et al. «Africanity as an Open Question.» Identity and Beyond: Rethinking Africanity. Discussion Paper 12. Uppsala: Nordiska Afrikainstitutet, 2001. 1924. Imprimé.

Barthes, Roland. Mythologies. 1957. Paris: Seuil, 2003. Imprimé.

Bérard, Stéphanie. «Déraillement, suspension, dislocation du dialogue ou l'impossible rencontre de l'autre... dans Blue-S-cat de Koffi Kwahulé». Africulures. Sans éd. 5 octobre 2006. Internet. 28 avril 2008 .

Bıntou. Texte Koffi Kwahulé. Mise en scène Laëtitia Guédon. Réal. Greg Germain. Dist. Annabelle Lengronne, Alexandre Jazédé, Olivier Desautels et Yohann Pisiou. RFO-Axe Sud. 2009. DVD.

Boëlle, Énora. «Fiche spectacle Big Shoot.» Africulures. Sans éd. Sans d. Internet. 28 avril 2008. Boucher, Denise. Les fées ont soif. 1978. Introd. Lise Gauvin. Montréal: L'Hexagone, 1989. Imprimé.

Breton, André. Position politique du surréalisme. Paris: Denoël, 1972. Imprimé. 
Césaire, Aimé. «Culture et colonisation.» Actes du $1^{\text {er }}$ Congrès International des Écrivains et Artistes Noirs. Présence africaine 8-10 (1956). 190-205. Imprimé.

Chalaye, Sylvie. «Africanité et création contemporaine.» Africultures 41 (2001): 7-14. Imprimé.

---. L'Afrique noire et son théâtre au tournant du XXe siècle. Rennes: PUR, 2001. Imprimé.

---. «À partir de combien de plumes au cul la chose est-elle crédible?»Oeuvres \& Critiques XXVI.1 (2001): 73-79. Imprimé.

---. «Briser l'enclos et donner rendez-vous ailleurs.» Africultures 41 (2001): 28-35. Imprimé.

---. Dramaturgies africaines d'aujourd'hui en dix parcours. Carnières: Lansman, 2001. Imprimé.

---. «Les masques de l’Africanité.» Africultures 41 (2001): 5-6. Imprimé.

---. «Le miroir inattendu des violences modernes.» Théâtre/Public 158 (2001): 35-40. Imprimé.

---. «Pour une africanité vagabonde. Entretien avec Koulsy Lamko.» Afrıcultures 41 (2001):

26-27. Imprimé.

---. «Théâtre africain et identité contemporaine.» Théâtre/Public 158 (2001): 86-91. Imprimé.

Couchard, Françoise. L'excision. Paris: PUF, 2003. Imprimé.

Deleuze, Gilles et Félix Guattari. Rhizome: Introductıon. Paris: Minuit, 1976. Imprimé.

Diawara, Gaoussou. Panorama critique du théâtre malien dans son évolution. Dakar: Sankoré, 1981. Imprimé.

Dionne, Olivier. «La structure de la subjectivité selon Hegel: Désir, Reconnaissance, Altérité.» Phares 4 (2004): Sans n. pag. Internet. 2 août 2010.

Diop, Boubacar Boris. «Identité africaine et mondialisation.» Africultures 41 (2001): 45-49. Imprimé.

Doyon, François. «L'origine gnostique de la vision négative de la sexualité chez saint Augustin.» Ithaque 1(2007): 25-47. Internet. 20 décembre 2009.

Du Bois, W. E. B.. The Souls of Black Folk. Oxford UP, 2007. MyiLibrary. Internet. 23 août 2010 . 
Fabre, Geneviève. «NOIR (théâtre noir aux États-Unis).» Dictionnaire encyclopédique du Théâtre. Éd. Michel Corvin. $2^{\mathrm{e} e ́ d . ~ V o l . ~ 2 . ~ P a r i s: ~ L a r o u s s e-B o r d a s, ~ 1998 . ~ I m p r i m e ́ . ~}$

Fanon, Frantz. Les damnés de la terre. 1961. Paris: La Découverte, 2002. Imprimé.

---. Peau noire, masques blancs. Paris: Seuil, 1952. Imprimé.

---. «Racisme et culture.» Actes du $1^{\text {er }}$ Congrès International des Écrivains et Artistes Noirs. Présence africaine. 8-10 (1956): 122-131. Imprimé.

Forestier, Georges. «Théâtre dans le théâtre.» Dictionnaire encyclopédique du Théâtre. Éd. Michel Corvin. $2^{\mathrm{e}}$ éd. Vol. 2. Paris: Larousse-Bordas, 1998. Imprimé.

Gates, Henry Louis. «Writing "Race" and the Difference It Makes.» Introduction. "Race," writing, and difference. By Gates. Chicago: U Chicago P, 1986. 1-20. Imprimé.

Genet, Jean. Les Nègres. Pour jouer Les Nègres. 1958. Paris: Barbezat-L’Arbalète, 1963. Imprimé.

Girard, René. Le bouc émissaire. Paris: Grasset, 1982. Imprımé.

---. Des choses cachées depuis la fondation du monde. Paris: Grasset, 1978. Imprimé.

---. Je vois Satan tomber comme l'éclair. Paris: Grasset. 1999. Imprimé.

---. La violence et le sacré. Paris: Grasset. 1972. Imprimé.

Glissant, Édouard. «Identité comme racine. Identité comme relation.»Identité, culture, développement. Colloque international sous l'égide du Conseil Régional de la Guadeloupe. Pointe-à-Pitre 11-13 décembre 1989. Paris: Éditions Caribéennes, 1992. 199-204. Imprimé. Goethe. Faust. Trad. Gérard de Nerval. Paris: Librio 1995. Imprimé.

Gilgenkrantz, Simone. «Nos ancêtres les cannibales.» M/S Médecine sciences 20.1 (2004): 14-16. Érudit. Internet. 4 novembre 2008.

Hall, Stuart. «The Question of Cultural Identity.» The Polity Reader in Cultural Theory, Oxford: Polity Press, 1994. 119-125. Imprimé. 
Hourantier, Marie-José. Du rituel au théâtre-rituel. Contribution à une esthétique négroafricaine. Paris: L'Harmattan, 1984. Imprimé.

Kilani, Mondher. «Crise de la «vache folle» et déclin de la raison sacrificielle.» Terrain 38 (2002): 113-126. Internet. 19 novembre 2009.

Kristeva, Julia. Pouvoirs de l'horreur. Essai sur l'abjection. Paris: Seuil, 1980. Imprimé. Kwahulé, Koffi. $1+1=1$. 1982. Inédit. Imprimé.

---. «Ave Maria». Regards-9. Carnières: Lansman, 2008. 23-33. Imprimé.

---. Big Shoot. Paris: Théâtrales, 2000. Imprimé.

---. Bintou. 1997. Carnières: Lansman, 2003. Imprimé.

---. Blue-S-cat. Montreuil-sous-Bois: Théâtrales, 2005. Imprimé.

---. Cette vieille magie noire. Carnières: Lansman, 1993. Imprimé.

---. «Du poétique au politique.» Colloque international Kwahulé : frères de son, frères de scène. Voix chœur à corps. Par l'Université de Paris III - Sorbonne Nouvelle et l'Université de Paris IV- Sorbonne. Lavoir Moderne Parisien. Paris. 12 avril 2008. Table ronde.

---. Entretien inédit. 26 août 2004.

---. Jaz. Montreuil-sous-Bois: Théâtrales, 1998. Imprimé.

---. Le Grand-Serpent. 1977. Inédit. Imprimé.

---. Misterioso-119. Montreuil-sous-Bois: Théâtrales, 2005. Imprimé.

---. Pour une critique du théâtre ivoirien contemporain. Paris: L'Harmattan, 1996. Imprimé.

Kwahulé, Koffi, et Gilles Mouëllic. Frères de son. Koffi Kwahulé et le jazz: entretiens. Montreuil-sous Bois: Théâtrales, 2007. Imprimé.

La Bible TOB. Toronto: Alliance Biblique Universelle, 1977. Imprimé.

La Haine. Réal. Mathieu Kassovitz. Dist. Vincent Cassel, Hubert Koundé et Saïd Taghmaoui. Mars Distribution. 1995. Film. 
Laplantine, François. «anthropophagique (mouvement).» Métissages. Dir. Laplantine et Alexis Nouss. Paris: Pauvert, 2001. 81-83. Imprimé.

Laurette, Pierre. «Poétiques et polyphonies francophones.» Préface. Poétiques et imaginaires. Francopolyphonie littéraire des Amériques. Dir. Laurette et Hans-George Ruprecht. Paris: L’Harmattan, 1995. 9-44. Imprimé.

Lefebvre, Joël. «GOETHE Johann Wolgang von.» Dictionnaire encyclopédique du Théâtre. Éd. Michel Corvin. $2^{\mathrm{e} e ́ d . ~ V o l . ~ 1 . ~ P a r i s: ~ L a r o u s s e-B o r d a s, ~ 1998 . ~ I m p r i m e ́ . ~}$

Lefeuvre-Déotte, Martine. L'excision en procès : un différend culturel? Paris: L'Harmattan, 1997. Imprimé.

Leiris, Michel. L'Afrique fantôme. 1934. Paris: Gallimard, 1981. Imprimé.

Lévi-Strauss, Claude. Anthropologie structurale. 1958. Paris: Plon, 1974. Imprimé. «Lycaon.» Le petit Larousse lllustré $2005,100^{\mathrm{e}}$ éd. Imprimé.

Maquet, Jacques. Africanité traditionnelle et moderne. Paris: Présence africaine, 1967. Imprimé. M’Baye, Ndongo. «À la recherche de 1'africanité.» Africultures 41 (2001): 36-38. Imprimé.

Nyamnjoh, Francis B, ed. et al. «Concluding Reflections on Beyond Identities: Rethınk Power in Africa.» Identity and Beyond: Rethinking Africanity. Discussion Paper 12. Uppsala:

Nordiska Afrikainstitutet, 2001. 25-33. Imprimé.

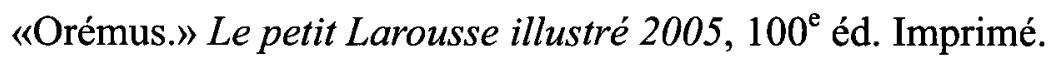

Pavis, Patrice. Dictionnaire du théâtre. Paris: Sociales, 1980. Imprimé.

Richon. Catherine. «Reproduire l'émotion de l'improvisation.» Fluctuat.net. 16 juillet 2006. Internet. 2 novembre 2009 .

Ruprecht, Alvina. «Blue-S-cat.» Franco-Théâtres. Sans éd. Juillet 2006. Internet. 2 novembre 2009.

Said, Edward. Orientalism. New York: Vintage, 1978. Imprimé. 
Said, Suzanne. «TRAGÉDIE.» Dictionnaire encyclopédique du Théâtre. Éd. Michel Corvin. $2^{\mathrm{e}}$ éd. Vol. 2. Paris: Larousse-Bordas, 1998. Imprimé.

Sartre, Jean-Paul. «Orphée noir.» Préface. Anthologie de la nouvelle poésie nègre et malgache de langue française. Éd. Léopold Sédar Senghor. 1948. Paris: PUF, 1969. xix-xliv. Imprimé. ---. Réflexions sur la question juive. 1954. Paris: Gallimard, 1995. Imprimé.

Scherer, Jacques. Le théâtre en Afrique noire francophone. Paris: PUF, 1992. Imprimé.

Schmidt-Brabant, Manfred. Les sept degrés de l'initiation. Le Faust de Goethe, un modèle primordial de l'initiation moderne. Trad. Joachim Berron. Montréal: DGP, 1998. Imprimé.

Senghor, Léopold Sédar. Les fondements de l'Africanité ou Négritude et Arabité. Paris: Présence africaine, 1967. Imprimé.

Soubrier, Virginie. «Dionysos africain: Étude sur le chœur dans Bintou \& Fama de Koffi Kwahulé.) Nouvelles dramaturgies d'Afrique noire francophone. Dir. Sylvie Chalaye. Rennes: PU Rennes, 2004. 141-149. Imprimé.

---. «Improvisation et politique. Réflexion sur le théâtre de Koffi Kwahulé.» Centre de Recherche sur l'Histolre du Théâtre. Université Paris IV - Sorbonne. Avril 2008. Internet. 24 avril 2008.

---. Koffi Kwahulé. Une voix afro-européenne sur la scène contemporaine. Position de thèse. Université Paris-Sorbonne, sans d. Fichier PDF.

---. Préface. Cette vieille magie noire. 1993. Par Koffi Kwahulé. Carnières: Lansman, 2006. 5-7. Imprimé.

---. «Vers une fraternité mystique universelle: l'«improviste» de Koffi Kwahulé.» Africultures 77 78 (2009): 191-201. Imprimé.

Tulard, Jean, éd. «VOIE LACTÉE (LA).» Guide des films 1895-1995. Édition du centenaire du cinéma. $2^{\mathrm{e}}$ éd. Vol. 2. Paris: Robert Laffont, 1995. Imprimé.

Ubersfeld, Anne. Les termes clés de l'analyse du théâtre. Paris: Seuil, 1996. Imprimé. 
Vaucher, Myriam. «Le cannibalisme bon à penser.» Le Courrier [Genève]. Le Courrier, 10 juillet 2009. Internet. $1^{\text {er }}$ novembre 2009.

Zadi, Grekou. «L'Abyssa dans la modernité.» Nzima-kotoko. Sans éd. 19 juin 2009. Internet. 25 août 2010 . 


\section{A) THÉÂTROGRAPHIE DE KOFFI KWAHULÉ}

\section{Les créations}

La mélancolie des barbares:

Créée le 26 septembre 2009 à la Maison des Jeunes de Rodez (France). Mise en scène de Sébastien Bournac (Compagnie Tabula Rasa). Distribution (adolescents du Grand Rodez): Lucie Assemat, Lucile Azam, Pierre Causse, Brice Denoyer, Marc EdmondGeorges, Ségolène Lachet, Alexandre Lisant, Margot Latasat, Judikäel Lemaire, Élise Revel, Catherine Delatre et Benoît Polylecot. Création sonore: Tom A. Reboul. Création lumières: Jean-François Chirac. (2009)

Les recluses:

Créée le 21 mai 2009 au Centre Culturel Français de Bujumbura (Burundi). Mise en scène: Denis Mpunga. Assitants à la mise en scène: Atome Diogène Ntarindwa, Robin Frédéric, Carole Karemera. Encadrement pédagogique: Carole Karemera. Traduction en kirundi: Docile Pacifique, Jeff Kalihabwa, Estelle Ndereyimana sous la direction de Maurice Mazunya et Atome Diogène Ntarindwa. La pièce a été traduite sous le titre de Abakubaguwe. Distribution: Funny Akimana, Domina Habonimana, Julienne Iciteretse, Nadine Irakoze, Fabiola Mukezamfura, Solange Ndakoraniwe, Yvonne Ndızeye, Joséphine Nibigira, Joselyne Nkundwanabake, Noëlla Nzeyimana. Scénographie, éclairages et costumes: Pierre Heydorff. Surtitrage: Fiona Irakoze. (2009)

Brasserie:

Créée en avril 2009 au Theater Brett de Vienne (Autriche) puis au Zold. Macska Diäkpance de Budapest (Hongrie). Création autrichienne et hongroise. Mise en scène: Yazid Lakhouache. Traduction hongroise: Rozsi Viktor Mark sous le titre de Sörfözde. Distribution: Judit Barkan, Csorba Sandor, Rozsi Viktor Mark et Trembeczki Peter. (2004)

Ave Maria:

Créée le 15 mai 2008 par le «Théâtre Niveau Parking» au Théâtre de la Bordée de Québec, dans le cadre du projet Regards-9 du Carrefour international de théâtre. Mise en scène: Michel Nadeau. Distribution: Marie-Josée Bastien, Réjean Vallée et Vincent Champoux. (2007-2008)

Train bleu:

Créée le ler février 2008 en appartement, Hôtel Beaulaincourt / Café Le Mac Ewans à Béthune (France). Création de la «Compagnie de Béthune» dans le cadre du projet On n'arrête pas le progrès. Mise en scène: Blandine Savetier. Distribution: Catherine Pavet et Bruno Tuchszer. (2007) 
Misterioso-119:

Créée le 24 avril 2007 au Théâtre Marni à Bruxelles (Belgique). Une création de la «Compagnie Kinesis». Mise en scène: Alex Lorette. Distribution: Hélène Couvert, Alexia Depicker, Daniela Ginevro, Cachou Kirsch, Justine Venet et Françoise Walot. Scénographie: Hélène Kufferath. Musique: Arnaud Blanpain. (2004)

Blue-S-cat:

Créée le 7 juillet 2006 à la Chapelle du Verbe Incarné à Avignon (France). Mise en scène: Koffi Kwahulé, assisté de Sébastien Rajon. Distribution: Olivier Brunhes et Nanténé Traoré. Scénographie: Christian Tirole. Lumières: Bastien Courthieu. Chorégraphie: Philippe Fialho. Création son: Gilles Normand. Costumes: Hicham Riffy. (2004)

La dame du café d'en face:

Créée le 21 janvier 2004 par le Theater Zuidpool d'Anvers (Belgique). Mise en scène: Johan Heldenberg. Traduction en flamand: Eva Schram sous le titre De Madam van't café van hierover. Distribution: Griet Debacker, Joke Devynck, Mike Libanon, Jobst Schnibbe, Eva Schram et Bob Snijers. (1998)

Scat:

Créée en septembre 2003 à la Comédie de Saint-Étienne (France). Mise en scène: Yves Bombay. Distribution: Jean-Pierre Laurent et Flora Brunier. Décors: Jacques Mallon. Costumes: Ouria Dahmani-Khoulhi. Régie: Patrick Falcon. (2003)

Big Shoot:

Créée le 2 mai 2003 au Théâtre du Grütli de Genève (Suisse). Mise en scène: Sandra Amodio. Distribution: Fabien Ballif et Antoine Richard. Conception: Fabien Ballif, Antoine Richard, Sandra Amodio. Scénographie: Nicole Grédy. Mouvement: Chiharu Mamiya. Création sonore: Vincent Hänni. Images: Dimitri Delcourt. Objets Sonores: Jean Faravel. (1999)

Histoires de soldats ou Le Masque boiteux:

Créée le 11 novembre 2002 au festival Novart de Bordeaux, présentée au Glob Théâtre de Bordeaux (France). Mise en scène: Souleymane Koly et Alougbine Dine. Distribution: François Chicaïa, Limengo Benano Melly, Anne Saffore, Bruno Lecompte, Karim Rande et Perrine Fifadji-Lanse. Photographies: Hervé De Williencourt. Décors: Jean-Loup Walraet. Costumes: Karlando. Musique: Serge Moulinier. Lumières et son: François Giraud. Vidéo: Sylvain de Zangroniz. (2001) 
P'tite-Souillure:

Créée le mai 2002 au festival Frictions organisé par le Théâtre Dijon Bourgogne

(France). Une création du «Maski Théâtre». Mise en scène: Serge Tranvouez.

Distribution: Isabelle Cagnat, Isabelle Védie, Victor de Oliveira et Jean-Paul Dubois.

(1999-2000)

Jaz:

Créée le 10 juillet 2000 par le Teatro Fontanonel de Rome (Italie). Une production de la «Compagnie Alcantara». Mise en scène: Daniela Giordano. Traduction en italien: Gianni Poli sous le titre de Jaz. Distribution, décors et costumes: Daniela Giordano. Musicien: Guido Giordano. (1998)

Il nous faut l'Amérique:

Créée en juillet 2000 au Pulsion Théâtre au Festival d'Avignon Off (France). Une création de la compagnie «Théâtre de la Mouvance». Mise en scène: Yves Sauton. Distribution: Sophie Heynssens, William Lassi et Yves Sauton. Décors: Mouvance Décors. Lumières: Jean-François Huchard. (1990)

Fama:

Créée en septembre 1998 au Centre culturel Français d'Abidjan (Côte d'Ivoire). Pièce librement inspirée des romans Les Soleils des Indépendances et Monnè, outrages et défis d'Ahmadou Kourouma. Mise en scène: Koffi Kwahulé. Distribution avec les comédiens de la compagnie Ymako Téatri: Vaber Douhouré, Claude Gnakouri, Luis Marquès, Dji Nésséré, Nathalie Atou Ekaré, Bernadette Oulaï Taha, Allassane Touré, Clémentine Papouet, Mathurin Nahounou, Mouna N'Diaye, Koffi Kwahulé, Diakité Satigouma, et Rahima Koné. Scénographie et costumes: Claude Goyette. Création lumières: Daniel Guillemant. Musiques: Diakité Satigouma et Rahima Koné. Régie: William Brou. (1995)

Village fou ou Les Déconnards:

Créée le 10 juillet 1998 à La Chapelle du Verbe Incarné à Avignon (France). Une production de «Khépri Créations / Afrique en créations». Mise en scène: Sidiki Bakaba et Koffi Kwahulé. Distribution: Sidiki Bakaba. Décors: Aurélia Fronty. Lumières: Kodjo Ebouclé. (1997)

\section{Les Créanciers:}

Créée en mai 1998 par la compagnie «Nord Ouest Théâtre» (théâtre forain itinérant, Caen en Normandie (France)). Mise en scène: René Pareja. Distribution: La Famille Magnifique. Dramaturgie: Gilles Boulan. (1997) 
Bintou:

Créée le 12 novembre 1997 au Théâtre International de Langue Française de Paris (France). Mise en scène: Gabriel Garran avec la collaboration de Pascal N'Zonzi. Distribution: Gladys Arnaud, Mohamed Aroussi, Catherine Bolanga-Campana, Delphine Clairice, Gora Diakhaté, Anouk Halter, Samuel Légitimus, Myriam Loucif, Aïssa Maïga, Emeric Marchand, Pier Ndoumbé, Nanténé Traoré et Solal Valentin. Direction technique: Georges Chaillan. Lumières: David Thomas-Collombier. Son: Vincent Hulot. (1996)

Cette vieille magie noire:

Créée le 6 novembre 2007 à l'Atelier du Plateau de Paris (France). Mise en scène: Claude Bokhobza. Distribution: Jocelyn Lagarrigue, Sylvie Goussé, Virgile M'fouilou, Koffi Kwahulé, Valérie Bélinga, Stéphane Delbassé, Claude Bokhobza, Michel Barbaud et Sébastien Brun. Scénographie: Michel Jacquelin. Écriture musicale: Michel Barbaud et Sébastien Brun. Lumières: Lionel Doucet. (1991)

$1+1=1$ :

Créée en 1982 à l'Institut National des Arts d'Abidjan (Côte d'Ivoire). Mise en scène de Guédéba Martin. Distribution: «Les Étudiants Ivoiriens en arts dramatiques à Paris». ' La pièce a été enregistrée par la télévision ivoirienne en 1983 et radiodiffusée par Radio France Internationale en 1983. (Inédite, 1982)

\section{Le Grand-Serpent:}

Créée en juillet 1981 au Centre Culturel de Treichville à Abidjan (Côte d'Ivoire). Mise en scène: Guédéba Martin. Distribution: «Les Étudiants Ivoiriens en arts dramatiques à Paris». ${ }^{2}$ La pièce a été censurée par les autorités après une seule représentation. Toutefois, elle a été radiodiffusée par Radio France Internationale. (Inédite, 1977)

Pièces publiées qui n'ont pas été jouées:

Ces gens-là (2003)

Goldengirls (2003)

1 «Les Étudıants Ivoırıens en arts dramatıques à Parıs» aussı appelés «Les Étudıants de Parıs» étaıt un groupe formé entre 1979 et 1982 En 1978, des élèves de l'École Nationale de Théâtre d'Abıdjan sont acceptés à l'École Nationale Supérıeure des Arts et Technıques du Théâtre de Parıs ou Centre de la rue Blanche. Il s'agıt de Tindılé Danıel (du Bénın), Lucıe Dagry, Antoine Soudé, Grahouan Guédéba Martın et Beugré Boıgnan Abou Bacar Touré et Koffi Kwahulé arrıvent également au Centre de la Rue Blanche Une vıngtaıne d'anciens élèves d'Abıdjan fréquentent les Cours Pérımonı et Brıgau de Parıs L'objectıf est le même pour tous: contınuer d'apprendre leur métıer en vue de la Troupe Nationale D’après Koffi Kwahulé, Pour une critıque du théâtre ivotrien contemporain (250)

2 Voir note 1 
El Mona:

Mise en lecture en 2001 par Jean-Michel Coulon dans le cadre de "Avril des auteurs» au Théâtre les Fédérés de Montluçon (France). (2001)

Une si paisible jolie petite ville (2001)

...Et son petit ami l'appelait Samiagamal:

Mise en lecture par Koffi Kwahulé en mai 1999 au Studio-Théâtre de la ComédieFrançaise à Paris (France). (1997)

Pièce inédite qui n'a pas été créée

Armageddon:

Mise en lecture en 1999 par Jean-Claude Berutti au Théâtre du Peuple de Bussang (France). (1997)

\section{Adaptations réalisées par Koffi Kwahulé}

Les travaux d'Ariane:

D'après la nouvelle du même titre de Caya Makhélé, adaptée et mise en scène en octobre 2000 par Koffi Kwahulé au Théâtre de la Verrière de Lille (France). Distribution: Maïmouna Coulibaly.

Blues pour Sonny:

D'après la nouvelle Sonny's Blues de James Baldwin, adaptée par Koffi Kwahulé. Une production de la Compagnie «Axe Sud». Créée en juillet 2000 au Festival d'Avignon Off à la Chapelle du Verbe Incarné. Mise en scène: Greg Germain, assisté d'Isa Armand. Distribution: Gora Diakhaté et Ludovi Signolet. Décor et costumes: Érick Plaza-Cochet. Lumières: Nasser Hammadi. 


\section{Pièces écrites en collaboration (créations)}

Profils atypiques:

Ecriture collective avec Nadège Prugnard et Louis Dominique Lavigne. Créée le 18 août 2010 au Lavoir Moderne Parisien. Coproduction de la Compagnie «Graines de Soleil» (France), Coopérative «Les ViVaces» (Québec) et Collectif «Éclats de Lune» (Maroc). Mise en scène de Khalid Tamer et Julien Favard. Distribution: Annick Fontaine, Angélique Boulay, Jean-Léon Rondeau, Zakariae Heddouchi et Olivier Parisis. Scénographie: Virginie Chevalier. Chorégraphie: Justine Favard. Création lumière: Antoine Cherix. Son: Samuel Favart-Mikcha. (2009)

Aziou Liquid:

Écriture collective avec Olivier Brunhes et François Prodromidès. Créée le 30 janvier 2007 au Théâtre Berthelot de Montreuil. Création de la «Compagnie l'Art Éclair» (2007). Mise en scène: Olivier Brunhes, assisté d'Aurélia Tastet. Distribution:

Stéphanie Lanier, Emmanuelle Bougerol, Isabelle Sueur, Isabelle Decroix, Virginie Cuisinier, Faouzia Boquet, Hélène Théry, Antoine Brugière, Philippe Dormoy, Alain Dumas, Johann Abiola, Bernard Boey. Scénographie: Bastien Courthieu et Corrine Véron-Durand. Chorégraphie: Tom Yang. Lumière et son: Bastien Courthieu et Gilles Normand. (2007)

Cocody Johnny:

Écriture collective avec Souleymane Koly. Commande de l'Ensemble Kotéba d'Abidjan (Côte d'Ivoire). Comédie musicale créée en mars 2004 à L'Hippodrome de Douai, Scène nationale. Mise en scène: Souleymane Koly. Elle a été adaptée au cinéma par le réalisateur Cheick Amadou Tidiane Seck. Dramaturgie et mise en scène: Koffi Kwahulé.

Les Troyennes et leurs soeurs:

Écriture collective avec Jacques Séréna, Nocky Djedanoum et Claude-Henri Buffard. Commande de la «Compagnie des Inachevés» de Grenoble (France). Créée en 2002 au Festival des réalités de Bamako (Mali). Mise en scène: Moïse Touré. Distribution: Jacques Séréna, Nocky Djedanoum, Claude-Henri Buffard.

Cavéo! ou À la recherche du centre perdu:

Écriture collective avec Arlette Namiand, Mohamed Rouhabi et Eugène Durif. Commande du Centre Dramatique National de Montluçon (France). Créée en juillet 2001 au Théâtre Hérisson de Montluçon. Mise en scène: Catherine Beau, Mohamed Rouhabi et Jean-Paul Wenzel. 
Pièce écrite en collaboration (inédite)

Tarjsa:

Écriture collective avec Michèle Bruhat. Commande de Gang des funambules. (inédite, 2002)

\section{Autre texte de Koffi Kwahulé qu'il a lui-même adapté pour le théâtre}

Babyface:

Nouvelle et roman de Koffi Kwahulé. Mise en jeu en 2000 par l'auteur lors des «Rencontres d'été de la Chartreuse» de Villeneuve lez Avignon (France). (2000)

\section{Koffi Kwahulé au Québec et en Ontario}

Bintou:

Lecture-spectacle en mai 1997 au Monument National (Montréal) dans le cadre du Festival de théâtre des Amériques. Avec Michelle Rossignol.

Big Shoot:

Lecture-spectacle le 30 septembre 2002 par le Théâtre Pàp à l'Espace Go (Montréal). Sous la direction de Kristian Frédric. Avec Sébastıen Ricard et Daniel Parent.

Big Shoot:

Création présentée du 6 septembre au ler octobre 2005 à la salle Fred-Barry du Théâtre Denise-Pelletier (Montréal). Coproduction du «Théâtre Denise-Pelletier» de Montréal et la compagnie «Lézards qui bougent» de Bayonne (France). En collaboration avec Scène nationale de Bayonne et du Sud Aquitain, Amis du Théâtre Populaire de la Côte Basque, Scène Nationale d'Albi. Mise en scène: Kristian Frédric, assisté de Dounia Bouhajeb. Distribution: Sébastien Ricard (Stan) et Daniel Parent (Monsieur). Conseiller à la dramaturgie: Denis Lavalou. Traduction des textes en hébreu: Hervë Elie Bokobza, Adam Almoni. Scénographie: Enki Bilal. Collaboration conception décor: Charles-Antoine Roy. Collaboration conception costumes: François Saint-Aubin. Éclairages: Nicolas Descoteaux. Chorégraphie des combats: Huy Thong-Toan. Effets spéciaux: Jean-Marc Riquier. Vidéo: Le Bureau officiel (Montréal). Conception sonore: Larsen Lupin (Montréal). Maquillages et tatouages: Jean Bégin. Photographie: Robert Etcheverry.

Tournée à Québec: du 4 au 9 octobre 2005 au Théâtre du Périscope. Même équipe qu'à la création à l'exception de Sébastien Ricard qui est remplacé par Stéphane Simard (Stan). 
Tournée à Ottawa: du 15 au 18 février 2006 à La Nouvelle Scène. Un accueil du Théâtre la Vieille 17. Même équipe qu'à la création à l'exception de Sébastien Ricard qui est remplacé par Stéphane Simard (Stan).

Tournée en France: mars, avril et mai 2006.

Reprise à Montréal: du 17 au 28 avril 2007 à l'USINE C. Même équipe originale qu'à la création.

Ave Maria:

Créée le 15 mai 2008 par le «Théâtre Niveau Parking» au Théâtre de la Bordée à Québec dans le cadre du projet Regards-9 du «Carrefour international de théâtre». Mise en scène de Michel Nadeau. Distribution: Marie-Josée Bastien, Réjean Vallée et Vincent Champoux (entre autres).

Reprise du 28 octobre au 22 novembre 2008 au Théâtre de la Bordée. Mise en scène: Michel Nadeau, assısté de Hugues Frenette. Distribution: Marie-Josée Bastin, Réjean Vallée et Vincent Champoux (entre autres). Scénographie: Pierre Thibault et Julie Pilote. Costumes: Virginie Leclerc. Musique et environnement sonore: Marc Vallée. Éclairages: Christian Fontaine. Projections: Mario Villeneuve. Accessoires: Julie Lévesque.

Profils atypiques:

Tournée à Montréal: du 19 au 30 octobre 2010 au théâtre Prospero. Même équipe qu'à la création en août 2010 à Paris. (Pièce écrite en collaboration)

$J a z:$

Création présentée du 4 au 15 décembre 2010 au Théâtre Les Deux Mondes à Montréal. Une production de la compagnie de théâtre «Les Deux Mondes» (Montréal) et de la compagnie «Lézards qui bougent» les Hauts de Bayonne (France). En coproduction avec l'USINE C, Scène nationale de Bayonne - Sud Aquitain, Théâtre à Châtillon, Théâtre Georges-Leygues, TOMA (Théâtre d'outre-mer en Avignon - Chapelle du Verbe Incarné), L'Espace Nuithonie, L'Oriental-Vevey, le Thêâtre Toursky, L'Office artistique de la Région Aquitaine - OARA. Mise en scène: Kristian Frédric, assisté de Margot Châron. Distribution: Amélie Chérubin-Soulières. Conception décor et costume: Kristian Frédric. Conception robotique et vidéo: Simon Laroche. Conception vidéo: Yves Dubé. Lumières: Nicolas Descôteaux. Effets spéciaux: Guy Fortin. Conception sonore et musicien: Michel Robidoux. Direction technique et de production: Eric Lapointe. Chorégraphie: Laurence Levasseur. Assistants aux costumes: Marilène Bastien et Julie Emry. Assistant vidéo et tournages: Christian Pomerleau. Assistant décor: Stéphanie Delarue. 


\section{B) ENTRETIEN AVEC KOFFI KWAHULÉ}

Entretien inédit réalisé avec Koffi Kwahulé à Paris, le 26 août $2004 .^{3}$

\section{Pourquoi avoir surtout choisi l'écriture théâtrale puisque vous êtes également comédien de formation et metteur en scène?}

Pour m'amuser... Je voulais savoir si je pouvais écrire. Mon premier texte a été Le Grand-Serpent (1977). Puis, j'ai été étonné de voir que la pièce avait intéressé mon professeur de littérature dramatique, à Abidjan. Le Grand-Serpent a été montée en Côte d'Ivoire en 1981, mais a été censurée après une seule représentation. Ensuite, Guédéba Martin, le metteur en scène du GrandSerpent, m'a demandé d'écrire quelque chose pour nous deux. Je n'avais jamais écrit de pièces pour deux comédiens car, en Afrique noire, un dialogue à deux n'existait pas pour la scène; on considérait cela comme un truc de Blancs. J'ai alors écrit $l+1=1$, une pièce à deux personnages. C'était la première fois qu'un auteur négro-africain se risquait à ce genre de pièce; généralement on préférait les pièces à plusieurs personnages, entrecoupées de chants et de danses. La pièce a pourtant été un succès et est même devenue une référence pour les gens de théâtre en Côte d'Ivoire. Ensuite, je n'ai plus rien écrit entre 1981 et 1989; je venais de terminer ma formation de comédien et je terminais un doctorat à la Sorbonne Nouvelle (Paris III). Une fois sur le marché de l'emploi, je me suis rendu compte que les rôles ne se bousculaient pas, surtout pour un comédien Noir. J'ai donc décidé de m'écrire des rôles. En fait, j'écrivais surtout par désœuvrement. J'ai terminé très rapidement Cette vieille magie noire qui a remporté le Grand Prix du concours RFI (Radio France Internationale), ce qui m'a permis de gagner 20000 francs français plus 100000 francs en bourse d'études, soit beaucoup d'argent à l'époque, en tout cas pour moi.

3 Certaınes précısıons ont été apportées par Koffi Kwahulé le 20 septembre 2010, par courrier électronıque 


\section{Est-ce que cet événement a confirmé votre vocation d'auteur dramatique?}

Non! Je me suis mis à enseigner pendant quelques années à Paris, dans des écoles supérieures privées de 1989 à 1994. Mais, dans le petit milieu du théâtre, mon nom circulait à la suite du prix RFI. Aussi m'a-t-on passé commande pour une pièce, ...Et son petit ami l'appelait Samiagamal, une pièce d'une trentaine de minutes. ${ }^{4}$ Dans la foulée, j'ai été invité en résidence au $13^{\mathrm{e}}$ Festival International des Francophonies en Limousin (1996). C'est à ce moment-là que Gabriel Garran", qui était dans le jury de ...Et son petit ami l'appelait Samiagamal, m'a dit qu'il fallait en faire une pièce plus grande. C'est ainsi que ...Et son petit ami l'appelait Samiagamal est devenue Bintou.

\section{Donc, c'est à ce moment-là que vous vous êtes dit que vous étiez vraiment un auteur} dramatique?

Oui, par la force des choses, puisque les autres me voyaient annsi. J'ai alors compris pourquoi les écrivains sont obsédés par les prix. Ils ont tellement besoin d'être rassurés que le prix devient une sorte de diplôme qui confirme la valeur de leur travail. Si je me suis finalement accepté comme auteur, c'est surtout grâce aux résidences, aux commandes et aux commentaires des autres écrivains.

\section{Maintenant que vous êtes un auteur dramatique, quelle vision du monde désirez-vous} exprimer par l'entremise de vos pièces?

Avant de chercher à exprimer une vision du monde, je suis d'abord interpellé par un type de théâtre. J'ai été nourri de contes africains et de cinéma hollywoodien. Ces formes racontent des histoires avec des personnages. Or, lorsque je suis arrivé en France, on m'a dit que tout cela était fini et qu'il était dorénavant ringard de raconter des histoires au théâtre. Malgré tout, j'ai voulu

4 Et son petıt amı l'appelatt Samiagamal a été écrite en 1996 et édıtée dans le recueıl Brèves d'alleurs aux éditıons Actes Sud-Papıers (Parıs). Il s'agıssait d'une premıère versıon partielle de la pièce Bıntou

5. Gabriel Garran est metteur en scène et fondateur du Théâtre Internatıonal de Langue Françaıse à Parıs 
écrire des histoires avec des personnages. Je pense que j'ai eu une bonne intuition car aujourd'hui, les auteurs dramatiques retournent aux récits. Écrire des histoires, cela signifie tout simplement que je n'ai pas créé le monde et que tout ce que je peux faire, c'est raconter le monde par le thêâtre. Autrement dit, on peut donner l'illusion qu'on structure le monde à nouveau et qu'on met fin au chaos. Est-ce parce que je viens d'un pays du Tiers-Monde que j'ai besoin de cohérence? Dans un certain milieu en France, quand on décrétait qu'il n'y avait plus d'histoires à écrire, plus de personnages à fabriquer, on décrétait inconsciemment que l'Histoire avait eu lieu. Et moi, venant du Tiers-Monde, j'étais au commencement du monde. Je ne pouvais pas accepter que le monde dans lequel je vivais avait déjà eu lieu. Il me fallait continuer à raconter des histoires, à refaire le monde et créer quelque chose de nouveau. Car sans histoires, tout s'arrêtait. Le théâtre tournait à vide. Il n'y avait plus de questions à se poser. Je pensais que pour moi, il était indispensable de continuer à raconter une histoire ou l'Histoire. C'était ma vision du monde au départ. Étrangement, aujourd'hui, je ne suis plus obsédé par le récit, et je préfère me consacrer davantage à la structure de mes œuvres. Avant, c'était le récit qui importait et maintenant, c'est la structure de la pièce qui prime.

\section{Au sujet de la structure, quelle est la différence entre une démarche dramaturgique conçue à partir d'une histoire à raconter et une démarche textuelle conçue à partir d'une structure qui vous interpelle?}

En fait, c'est un peu comme un musicien qui trouve une mélodie avant de se préoccuper des paroles qui iront avec. Dans mes premières pièces (Le Grand-Serpent, $1+1=1$, Cette vieille magie noire, Bintou, Fama) toute mon énergie était tendue par le fait de trouver des péripéties pour rendre l'histoire intéressante. Aujourd'hui, avec l'influence du jazz où les musiciens utilisent tous le même thème (la même histoire) pour donner libre cours à leur propre son, les histoires sont devenues presque squelettiques dans mes pièces; on peut les résumer en une seule phrase. 
L'histoire n'est plus qu'un prétexte (le motif) pour tenter des formes. Par ailleurs, le fait que depuis 1996 (depuis Bintou) je n'écris presqu'exclusivement que des commandes n'a fait que renforcer ce penchant. Même si les commanditaires ne m'imposent pas tous un thème, une commande reste une commande. Aussi me suis-je rabattu sur les questions formelles, puisque les commanditaires, s'il leur arrive de me donner des contraintes (thème, nombre de personnages...) ne se préoccupent jamais de la forme. Du coup, la structure est devenue mon jardin secret au détriment du récit. C'est ainsi que depuis la commande que m'a passée en 2008 le théâtre «Niveau Parking» de Québec dans le cadre du $400^{\circ}$ anniversaire de la ville, j'ai écrit trois autres pièces à partir de ce même texte, avec la même histoire, les mêmes personnages. Un peu comme un musicien jouerait plusieurs versions de Round Midnight.

\section{Dans vos pièces, on observe à maintes reprises que vous mettez en scène des luttes de} pouvoir entre des individus. Pourquoi?

Je pense que le théâtre est l'art de se confronter à l'altérité de l'autre et des risques qu'elle implique nécessairement. Avec le théâtre, il faut qu'il y ait quelqu'un en face. Tous les grands textes de théâtre reposent sur la notion de conflit. Le théâtre est d'abord conflit. Les personnages et les comédiens inconsciemment essaient de mordre sur l'espace de l'autre et, dans mes pièces, les personnages sont toujours en train d'empiéter sur l'espace des autres même lorsque le conflit n'est pas apparent.

\section{Comment les metteurs en scène doivent-ils interpréter les didascalies ${ }^{6}$ qui se trouvent dans vos pièces?}

6 Les didascalıes comprennent les indicatıons scénıques (de lieu et de temps) et les indıcatıons données aux comédiens (parole et gestuelle) 
J'ai cessé de faire de la mise en scène depuis la fin des années 1990 avec Fama ou Les

Déconnards, je ne me souviens plus laquelle. Je mettais beaucoup d'indications scéniques et je me suis rendu compte que c'était des indications de mise en scène parce que, par anticipation, je faisais la mise en scène. D'une certaine manière, j'écrivais des spectacles et non des pièces de théâtre. Maintenant, j'essaie d'éviter cet écueil. Par exemple dans Misterioso-119 (2004), il n'y a même plus de didascalies. Mais, en même temps, dans Blue-S-cat, le texte qui précède Misterioso-119, il y a beaucoup de didascalies. Les didascalies sont des fenêtres qu'on ouvre dans une pièce. Alors que les indications scéniques, je les prends au sens de directions. Elles indiquent une direction. C'est une fenêtre qu'on ouvre pour le metteur en scène. Ça n'est pas une donnée coercitive, et c'est pour cela que dans la pièce $J a z$, j'ai écrit «peut-être» ${ }^{7}$ pour que celui qui fait la mise en scène fasse ce qu'il veut. S'il veut monter l'œuvre différemment, je sais à partir de quelle référence il réalise son travail. En fait, la didascalie est peu à peu devenue, dans mes textes, une parenthèse hypothétique. Misterioso-119 est ma première pıèce sans didascalies, sans indications scéniques et sans personnages parce que tout se déroule en prison, espace sans «fenêtres» et sans «1dentités».

\section{Dans Cette vieille magie noire, vous indiquez qu'un quartet de jazz est indispensable.}

\section{Pourquoi?}

Parler d'un quartet de jazz provoque une impression, des images. J'écris en France, mais si j'écrivais aux États-Unis, ce serait peut-être différent. En France, le jazz ne provoque pas d'images. Je suis obligé de rappeler aux metteurs en scène que le quartet de jazz suppose ceci et cela. Le jazz est une musique, si j'ose dire, de chair et de son, une musique qui est associée à la présence concrète du corps, ici et maintenant, comme le théâtre. Cette vieille magie noire ne s'appuie pas que sur la magie du théâtre; elle est traversée également par le théâtre du jazz, car

7 «Une femme / Le crâne rasé peut-être / Nue peut-être» (57). 
nous sommes en face d'une musique éminemment théâtrale, voire dramatique. Le quartet est un spectacle dans le spectacle. À ce titre, le quartet participe de la mise en abîme qui caractérise la pièce.

\section{Dans la pièce, quels sont les liens avec le cinéma (scénario et séquences filmées)?}

Dans Cette vieille magie noire, les personnages sont inspirés du cinéma hollywoodien. J'avais besoin du déroulé du cinéma. Les descriptions ressemblent plutôt à des séquences cinématographiques qui correspondraient à un scénario. C'est le cinéma qui m'avait nourri. J'écrivais comme si je travaillais au cinéma, devant un écran avec une caméra.

\section{Qu'avez-vous pensé de la mise en scène de Greg Germain ${ }^{8}$ de la pièce Blues pour Sonny?}

Greg Germain vient un peu de la même culture que moi, une culture cinématographique. Il a été le premier acteur Noir connu en France. Il a fait du cinéma et de la télévision et c'est pour cela que, quand il fait du théâtre, il l'aborde comme s'il faisait du cinéma. Blues pour Sonny est fait de manière cinématographique, c'est-à-dire que le spectacle avançait par séquences, avec des ellipses, comme «monté». La facture d'ensemble très réaliste, notamment dans les costumes, renvoyait à l'atmosphère du cinéma hollywoodien des années 1950 . J'ai trouvé que c'était un très beau travail. Même si on n'avait pas l'impression qu'il innovait avec cette pièce, il a créé un spectacle qui a marché car c'était agréable à regarder. Quand on est nourri de cinéma hollywoodien, les gens ont un sens du plaisir, ils veulent donner du plaisir. C'est ce que fait Hollywood d'ailleurs. Ils ne veulent pas refaire le monde, même s'ils refont le monde sans vraiment le vouloir. Mon obsession est de donner du plaisir aux gens, sinon ça ne vaut pas la

8 Greg Germain est metteur en scène, acteur, auteur et réalısateur Il dırıge également les actıvités de la Chapelle du Verbe Incarné en Avignon. 
peine. Chez Greg, il y a également cette notion de plaisir. Mais ce n'est pas en France qu'on monte bien mes pièces. C'est surtout à l'étranger.

\section{Pourquoi?}

Pour des raisons politiques et culturelles. Politiques parce qu'en France, il y a ce qu'on appelle la francophonie. La francophonie, par définition, est un ensemble de pays où les gens parlent français mais ne sont pas de nationalité française. Quand il s'agit d'un auteur ivoirien, on monte la pièce à partir de l'idée qu'on se fait de ce que doit être un Ivoirien ou un Africain. Du coup, l'idée est fausse. Le résultat n'est pas mauvais, mais mitigé. Lorsque Greg Germain met en scène mon œuvre, il ne monte pas une pièce d'un Africain. Il monte une pièce, un point c'est tout. Quand je vais à l'étranger, plus cette culture est éloignée de la mienne, mieux on monte mes pièces. P'tite-soulllure a été montée en France et à Prague, et la production de Prague a été plus juste parce que le jeu était jubilatoire; on chantait et dansait non pas parce que ça faısait africaın, mais c'était du plaisir donné par des créateurs tchèques à des spectateurs tchèques à travers l'expérience vécue d'un certain Koffi Kwahulé.

\section{Toutefois, $P$ 'tite-souillure est une pièce sombre et difficile.}

Oui, mais ceux qui l'ont montée ne me connaissaient pas. Puisqu'ils ne m'avaient jamais vu, ils n'avaient pas d'a priori en ce qui me concerne. Ils n'ont pas pensé qu'il fallait trouver un langage «africain». J'ai vu La Dame du café d'en face chez les Flamands. C'est une pièce un peu statique, et là, c'était de la jubilation, du rire. Quand je les ai rencontrés, ils étaient surpris et ils me disaient qu'ils comprenaient finalement ce qui n'allait pas dans leur mise en scène. Peut-être m'avaient-ils trouvé triste et sinistre? En tous les cas, j’ai trouvé leur travail excellent et j'ai pensé: «Quelle chance qu'ils ne m'aient pas vu avant.» 


\section{Qu'avez-vous pensé de la mise en scène de Gabriel Garran au sujet de Bintou?}

Garran est celui qui, plus que moi-même, a cru en moi. C'est lui qui m'a fait comprendre que j'avais une langue. Et sa mise en scène, plutôt réaliste, avec des références propres aux personnes de sa génération (West Side Story), a cherché la meilleure façon possible de servir l'histoire et la langue de la pièce. Le succès aussi fulgurant qu'inattendu du spectacle m'a permis, par la suite, d'exister artistiquement. Voilà pourquoi j'ai toujours considéré Garran comme mon père spirituel au théâtre.

\section{Les lieux où se déroulent vos pièces sont souvent clos. Dans Bintou il y a l'appartement, le} terrain vague et le café. Dans Big Shoot, le lieu pourrait ressembler à une cage de verre. Dans Jaz, Misterioso-119 et Blue-S-cat, ce sont aussi des lieux clos. Ces espaces restreints sont bien sûr des métaphores. Que vouliez-vous signifier?

De manière générale, je crois que le lieu clos est récurrent dans mon théâtre probablement parce que je vis à l'étranger, même si je suis Français. À l'étranger, peu importe les conditions dans lesquelles ont vit, on sent l'enfermement de l'exil. Il y a aussi le fait que je suis Noir, et être Noir dans le monde, même en Afrique, c'est sentir qu'on vit dans un tout petit espace. C'est la sensation de vivre dans un monde qui semble très vaste, ouvert mais plus il est vaste et ouvert, plus on a l'impression de vivre dans un espace de plus en plus étroit. C'est une sensation physique aussi qui est exprimée à travers les lieux scéniques.

Prenez la sanisette de Jaz; elle représente le rétrécissement de la société, c'est la cathédrale qui se réduit à la dimension et à l'odeur d'une sanisette. Ce qui se passe dans la sanisette est un viol. Mais c'est un viol qui a lieu le dimanche, jour de la messe et donc il est d'autant plus transgressif. Et quand l'homme parle à Jaz, il exige de la retrouver le dimanche pour que le viol devienne une forme de cérémonie, voire une messe où la violée est la sacrifiée. Les inquisiteurs, ceux qui dirigent le monde, fonctionnent ainsi. Ils ont transformé le monde, représenté par la cathédrale, le 
temple, la synagogue ou tout lieu sacré que doit être le monde; soit tout lieu spirituel, un espace où l'être humain réifie l'Autre, le transforme en «chose». Les inquisiteurs imposent une nouvelle liturgie du monde et, dans ma pièce, tout cela se passe métaphoriquement dans une sanisette. Globalement, le monde est comme une sanisette. Je le «sais» dans ma chair et dans mon esprit, tout simplement parce que je suis Noir. On multiplie les moyens de communication mais étrangement, ils ne relient pas le monde. Nous avons l'illusion d'être ensemble mais, en fait, chacun est seul, lié à quelqu'un qui est tout aussi seul. Voilà ce que j'appelle la nouvelle liturgie du monde.

\section{Puisqu'on parle tellement d'«Africanité», dites-moi comment on définit un auteur dramatique africain?}

C'est d'abord politique. Et puis, pourquor les gens veulent-ils savoir si je fais du théâtre africain ou pas? Je participe à des rencontres avec des auteurs «français» entre guillemets puisque Blancs.

Je suis auteur français de toute façon. À moi, on me pose toujours la question de l'identité. Je n'ai pas plus de problème d'identité que le boulanger du coin. N'importe quel être humain a des problèmes d'identité. Tu sors du néant, tu existes, c'est tout. Au niveau métaphysique, tu es déjà dans une quête identitaire. On n'a pas besoin de moi pour se poser cette question. Réduire cela à la question Noire, africaine, c'est inconsciemment, pour l'Autre, essayer de se définir lui-même. Si ma place est clairement définie, la place de l'Autre est préservée. Ce qui nous fait peur c'est justement ce que j'appelle les contours flous du monde. Qui es-tu? Dans certaines de mes pièces, on ne sait pas toujours s'il s'agit d'hommes ou de femmes, de Blancs ou de Noirs. La différence est rarement spécifiée. Je le fais volontairement parce que je sais que cette attitude fait peur aux imbéciles, parce que les imbéciles ont besoin d'être assignés à résidence dans une identité, pour se définir eux-mêmes. Je pense qu'ils n'ont pas à s'appuyer sur moi pour se définir. Je ne cherche pas à faire d'eux des Blancs ou des Chinois. Je suis comme je suis, c'est tout. Pourquoi ont-ils 
absolument besoin de m'accoler une étiquette que trop souvent, ils ont eux-mêmes fabriquée? Il y a un proverbe chez nous qui dit: «Quand un aveugle te dit «lançons-nous des pierres», c'est parce qu'il a déjà le pied posé sur la pierre.» Quand quelqu'un t'incite à parler de ton identité, c'est qu'il a déjà ton identité préconçue et fabriquée. Et si tu fais quelque chose en dehors de ce qu'il a fabriqué, il va paniquer. Il va persuader les gens autour de toi en disant: «Attention à ce qu'il dit, car ce n'est pas un vrai, il ne sait plus qui il est.»

Je vais vous donner un exemple. Il y a des gens qui n'aiment pas ce que je fais. Ils disent: «Le théâtre de Koffi est un peu américain.» Comme on est en France, quand on dit américain c'est déjà un défaut, non pas parce qu'il est mauvais mais simplement parce qu'il renvoie à l'Amérique. Je suis allé à New York rencontrer les membres du Lark Theatre Company qui ont fait traduire plusieurs de mes textes, dont sept seront publiés en automne 2010 par Indiana University Press. Ils m'ont dit: «Tu sais ce qu'on aime dans tes pièces, c'est qu'elles sont très européennes.» Chaque fois que quelqu'un me dit que mon théâtre n'est pas africain, il claironne en réalité qu'il sait déjà ce que c'ést que «l'âme africaine».

On pense au cas du comédien, auteur et metteur en scène Wajdi Mouawad. Il est né au Liban, a étudié en France et vit maintenant au Québec depuis 20 ans. Il m'a déjà parlé de cette question d'identité en affirmant: «Au Québec, on me voit comme un auteur du Liban et au Liban, on me voit comme un auteur du Québec. Pourtant, il n'y a rien qui renvoie de manière claire au Liban dans mon théâtre.»

Mais lui, au moins, on lui dit parfois qu'il est auteur libanais. (Rires). Mon théâtre est africain, mais d'un «Africain étranger», c'est-à-dire de la diaspora, et ceux qui disent qu'il est américain ou européen ne disent rien d'autre. C'est un théâtre «étranger». Gabriel Garran m'avait déjà dit, à l'époque de Bintou, que ce que j'avais écrit était très bien, mais que la prochaine fois, il fallait que je parle un peu plus de moi. (Rires). Toute cette question ne me préoccupe pas. Je fais 
d'abord du théâtre ivoirien avec l'expérience de ce que je suis devenu. Je ne veux pas revendiquer que je fais du théâtre français, parce que la France n'en a pas besoin. Le répertoire ivoirien en a davantage besoin. J'ai grandi en Côte d'Ivoire, je suis venu en France à 23 ans pour poursuivre mes études. J'étais déjà formé. Les influences que j'ai subies par la suite ont été transformées par ce que j'étais déjà avec mon éducation et mes origines. Il faut que les gens finissent par accepter le fait que, peut-être, le théâtre africain est plus compliqué qu'on ne le croit.

\section{Comment expliquez-vous la présence du chœur et du coryphée dans certaines de vos pièces?}

\section{Est-ce un lien avec l'oralité du théâtre africain?}

Ça a commencé par une commande qui demandait plusieurs personnages. J'ai donc fabriqué un chœur. Ce n'était pas pour faire africain. Je pensais à Bintou comme une tragédie contemporaine. Il fallait convoquer des éléments de la tragédie classique pour parler d'une adolescente de 13 ans de la banlieue. Elle remplace les dieux, les demi-dieux, les demi-hommes, les seigneurs, les nobles, les guerriers et les reines qui sont au centre de la tragédie classique. Aujourd'hui, on ne peut plus percevoir la tragédie comme auparavant. C'est une perception qu'on a de la tragédie aujourd'hui qui peut naître dans une banlieue. Le chœur dans Bintou intervient d'abord comme signe, il dit: «Attention, c'est une tragédie.» Cependant, la structure de ce chœur diffère de celui de la tragédie grecque où il reste en dehors de l'action, contrairement au chœur de Bintou qui prend parti. Dans mes autres pièces, il y a des chœurs qui ne sont pas identifiés comme tels. La foule, le groupe sont des chœurs déguisés dans Cette vieille magie noire. Dans Mistérioso-119, il y a un chœur. C'est une seule personne, mais elle n'intervient pas même si elle est le personnage principal. Il s'agit de l'auteure (Elena Hebrayova) de la pièce que les personnages en prison tentent de monter. C'est inversé par rapport à un groupe. Là où il y a chœur dans mon théâtre, il y a tragédie. 


\section{Quelle est l'importance de l'oralité héritée de la tradition africaine?}

Je sais que d'une manière ou d'une autre, l'oralité va ressurgir sous une certaine forme, mais pas comme quelque chose qu'on peut identifier par rapport à la tradition orale africaine. Par exemple, dans Bintou, l'espace est construit de manière musicale. Il y a un rythme dans la succession entre espace ouvert et espace fermé. Le rythme n'est pas seulement dans la langue. Il se retrouve dans la construction de l'espace. Dans Cette vieille magie noire, il y a une succession de scènes où il y a deux ou trois personnages et les scènes de «foule». Donc, le rythme se retrouve autant dans l'espace que dans le texte et les voix parlantes.

Qu'en est-il au juste de toute cette question d'américanité dans vos pièces? On pense au jazz. Cette vieille magie noire signifie That Old Black Magic. Jaz c'est le jazz. Quel est votre rapport à l'idée qu'on se fait de la culture américaine?

Ce que j'exprime, c'est le mythe américain parce que les mythes me fascinent. C'est le seul qui reste après Dieu, parce qu'il semble convalescent, et le communisme s'est écroulé après la chute du mur. Il n'y a que celui-là qui reste. Le mythe américain est un mythe humain, de notre époque, fait avec des hommes de maintenant. On a besoin des mythes pour simplifier le monde, pour humaniser le monde et pour expliquer le monde. En même temps, l'Amérique est une superpuissance. Jamais aucun peuple n'a été aussi puissant. L'Amérique est devenue moi et tout le monde. C'est le seul pays où, au bout d'une semaine, je me sens chez moi. Je ne dis pas que les gens m'acceptent et me sourient. Non, mais dans ma tête j'ai l'illusion de me retrouver chez moi. L'Amérique est structurée avec la chair du monde qui est réunie sur ce territoire. C'est le pays de tout le monde. La première fois que je suis allé à New York, j'ai eu l'impression d'y avoir habité toute ma vie. Ce n'est pas seulement le mythe car j'aime aussi l'Amérique dans sa réalité quotidienne. Il y a les excès bien sûr mais les Américains ne se prennent pas la tête. Ils foncent. Les gens sont jeunes là-bas alors qu'ici, les gens sont tellement vieux. L'Europe est un vieux 
continent avec beaucoup de sagesse, mais on est un continent où les mentalités sont vieilles. L'Amérique, dans son fonctionnement même, reste jeune dans son rapport au monde.

\section{D'où vient ce désir de remettre en question le romantisme par rapport à l'Amérique?} Il ne s'agit pas d'une admiration béate. Et je ne suis pas d'accord avec tout ce qui s'y fait idéologiquement. Ce n'est pas cela. Ce que j'aime, c'est l'obsession du plaisir et du désir. Ils font les choses avec désir. Les peuples qui sont vieux n'ont plus de désirs. Ils sont cassés. Aussi meurent-ils lentement. Pour moi, il ne s'agit pas seulement des États-Unis. L'esprit américain se retrouve dans toutes les Amériques, du Canada jusqu'en bas du continent sud-américain. Il y a tout de même une certaine forme de romantisme et une certaine dose de naïveté chez moi lorsque je pense à New York, aux gens que j'y ai rencontrés.

Vos personnages parlent beaucoup de l'Amérique. Ils en rêvent. Dans Bintou, on sent cette influence qui passe par la musique et le cinéma. Big Shoot donne cette même impression. Oui, parce que l'Amérique, ou les Amériques, est devenue la seule fenêtre pour l'être humain. Pendant très longtemps, l'Europe était le modèle pour les gens du Tiers-Monde. Maintenant, c'est l'Amérique qui est lointaine et proche à la fois. Il y a le cinéma, la télévision qui nous inondent de l'Amérique. Mes personnages sont attirés comme par une boussole vers le nord de l'Amérique ou comme un tournesol par le soleil. J'ai une fascination pour l'Amérique et une répulsion également car il y a des éléments de cette culture que je ne comprends pas.

\section{Quels sont les auteurs dramatiques qui vous intéressent? On vous a comparé à Genet et à}

\section{Koltès notamment.}

J'aime les musiciens surtout. Comme auteur de théâtre, en France, j'aime Koltès, mais ce sont les musiciens de jazz qui m'intéressent avant tout. 


\section{Et que pensez-vous de l'auteur américain LeRoi Jones (Amiri Baraka)?}

Je l'ai déjà lu. J'ai même monté Cour royale et joué dans sa pièce Le Métro fantôme, en 1981, à (l'ancien) Théâtre Noir de Paris.

\section{Mais Le Métro fantôme, c'est un peu comme votre pièce Blue-S-cat où un homme et une femme sont coincés dans un ascenseur et elle finit par le tuer?}

Mais c'est vrai! C'est une pièce qui m'a beaucoup fasciné. L'idée de la sanisette de Jaz vient aussi probablement de là car, dans l'une de ses pièces, il est question des toilettes publiques. Il est l'une de mes influences en fait, mais il est devenu trop militant, trop manichéen par la suite.

\section{La métaphore du pouvoir revient comme un leitmotiv dans vos pièces. Pourquoi?}

Parce que les rapports de pouvoir sont ceux qui établissent les liens entre les individus. Dans Big Shoot, il y a le bourreau et la victime, deux êtres qui sont métaphoriques. Le tueur, c'est l'écrivain. Il s'agit, dans un premier temps, d'une pièce sur le processus de création; ensuite, la relation de pouvoir entre deux êtres. Le personnage de Monsieur est un tueur en série à l'envers puisque les gens viennent se faire tuer par lui. Il ne va pas les chercher. Il y a tout le côté cérémoniel du jeu de mise à mort. Monsieur est l'écrivain qui décide tout. Il invente Stan et lui donne un nom. Il développe même un rapport de sympathie avec lui pour mieux le tuer à la fin comme il a fait avec les autres avant lui. L'écrivain invente des personnages pour le plaisir de les trucider. Stan raconte ce que Monsieur veut bien entendre et parfois Monsieur lui indique ce qu'il doit raconter.

Évidemment, il est aussi question des Reality Shows. Big Shoot est aussi une pièce sur la crise de l'intimité. Dans ces émissions, ce sọnt des mises à mort volontaires et publiques parce que les 
gens ne sont pas obligés d'y participer. Tout le monde veut ses quinze minutes de gloire qu'annonçait Andy Warhol, même au prix de sa propre vie.

\section{On a dit de la pièce $J a z$ qu'elle était une métaphore du viol de l'Afrique. Est-ce le cas?}

C'est ce que certaines personnes plaquent sur mon texte. Je crois que c'est une lecture possible. Il ne s'agit pas du viol de l'Afrique, mais le jazz n'est-il pas aussi l'histoire d'une résistance au viol? Même si tous mes personnages peuvent êtres interprétés aussi bien par des Blancs, des Noirs ou des Jaunes, c'est d'abord l'expérience «Noire» qui est traduite dans toutes mes pièces. $J a z$, c'est d'abord le viol de l'homme contemporain à travers l'expérience vécue du Noir.

\section{Dans Blue-S-cat, comment voyez-vous le rapport entre l'homme et la femme?}

On parle de communication, de moyens de communication. Il y a donc une espèce de négation même du corps dans cette relation qui monte. Le corps de l'autre attire la première agression. L'ascenseur, par définition, fait qu'il y a deux corps en mouvement. Les deux corps sont soudainement dans cette proximité, cette promiscuité. La femme a peur de l'homme tout autant que l'homme a peur de la femme. Pour elle, ce sont des peurs associées au corps, au viol ou au meurtre. Même si elle ne dit pas ces mots, elle y pense. L'homme ressent aussi un malaise et pour tenter d'y remédier, il ne cesse de réciter des choses, essentiellement des chiffres, dans sa tête. Les corps ne sont plus habitués à être vraiment ensemble dans cette communication qui se fait désormais toujours à distance. Dans ce lieu clos, les corps sont réunis de manière fortuite au moment où l'ascenseur est bloqué et les corps coincés. Il y a des peurs qui naissent et ce sont ces peurs qui poussent la femme à tuer l'homme.

Dans votre livre Pour une critique du théâtre ivoirien contemporain, vous parlez d'un théâtre de «décolonisé». Comment voyez-vous toute la question coloniale dans votre écriture? 
J'essaie de m'en échapper car je suis conscient que je suis aussi un décolonisé. Dans décolonisé, il y a colonisé, toujours. C'est là que le mot décolonisé m'intéresse car j'essaie, dans mon théâtre justement, de dépasser cette question-là. C'est dangereux pour les Africains de penser toujours leur rapport au monde dans le contexte du début de la colonisation. Ça donne l'illusion que le monde a commencé avec la colonisation et qu'ils se réduisent eux-mêmes à d'anciens colonisés alors qu'ils sont actuellement au-delà de la colonisation. Je veux penser la colonisation comme une contingence de l'Histoire et non pas comme l'absolu de l'Histoire africaine. C'est un moment parmi des milliers de moments de l'Histoire. Dans notre mode de pensée actuel, on croit que c'est le moment absolu, mais il n'y a que les Francophones qui fonctionnent de cette manière-là. Les Anglophones ne fonctionnent pas ainsi. Ils n'en ont rien à faire de la colonisation. La Francophonie est toujours consciente du rapport colonial; c'est quelque chose qui est politique et non pas culturel.

\section{Mais les rapports coloniaux font tout de même partie de votre expérience.}

Bien sûr. Absolument. Mais je ne veux pas enfermer ce que j'écris dans des rapports coloniaux, même si des traces existent dans mon écriture. Je n'ai pas le choix. Cela fait partie de moi; je vis et j'écris avec ça.

\section{Plusieurs de vos personnages évoluent dans les rites sacrificiels. Pour quelles raisons?}

Jaz, Stan, Bintou, Shorty sont tous des êtres «sacrifiés» car le personnage qui me fascine le plus, c'est le Christ. Je suis chrétien sans aller à l'Église. Tous mes personnages sont des variations sur la figure du Christ. Bintou est le personnage qui se rapproche le plus du Christ même si c'est une petite peste. C'est un Christ à l'envers, un Christ déréglé. Mais Bintou a toutes les caractéristiques du Christ. Ce sont les autres qui la construisent par le verbe, par un nouvel Évangile. Il y a trois 
Évangiles ${ }^{9}$ dans la pièce: celui de Manu, celui de Blackout et celui de Kelkhal. Tout ce qu'on sait de Bintou, ce sont tous les autres qui le racontent.

Le sens de la vie, c'est le sacrifice de soi. Le Christ dit: «Je suis le fils de Dieu. Je peux me sauver de la croix, de la condition humaine, je peux tout faire. Mais le sens de la vie, pour que la parole soit pleinement accomplie, doit faire en sorte que j'arrive au sacrifice ultime qui est le sens de ma mission.» Évidemment, ça semble violent lorsqu'il dit qu'il doit mourir pour que la communauté humaine puisse continuer son aventure dans de meilleures conditions. Le Christ naît pour sauver le monde. De manière inconsciente, on est persuadé que l'innocence de l'enfant va nous sauver jusqu'à ce qu'il perde lui-même son innocence. Et voilà l'essentiel de certains de mes personnages.

9. Manu, son petıt amı, a arrêté de consommer du crack pour Bıntou (Elle accepte le commerce de la drogue mais refuse que son entourage y touche.) Blackout a tué un homme pour elle (Elle était fière du geste posé car elle n'a pas peur de la mort.) Kelkhal, le poète, parle de l'érotısme de Bintou et de la manière dont elle accomplıt sa danse du ventre (Elle veut incarner Samıa Gamal et parvenır à «danser avec le nombrıl quı tourne sur luı-même» (Bıntou 15)) 